Carlos Eduardo de Brito Novaes

\title{
Métodos de estimação de derivadas via cálculo operacional e aplicações a problemas de controle
}




\section{Carlos Eduardo de Brito Novaes}

\section{Métodos de estimação de derivadas via cálculo operacional e aplicações a problemas de controle}

Dissertação apresentada à Escola Politécnica da Universidade de São Paulo para obtenção do grau de Mestre em Engenharia.

Área de Concentração: Engenharia de Sistemas.

Orientador: Prof. Dr. Paulo Sérgio Pereira da Silva.

São Paulo 


\section{Dedicatória}

Dedico este trabalho aos amigos que me incentivaram, à minha família que se resignou com alguns momentos de ausência, ao meu pai que por muitas vezes exclamou: - De novo nestes gráficos! Quando isto termina? - E que em tantas outras vezes me apoiou, acompanhou em viagens e apresentações e deu seus palpites na construção do mecanismo.

Agradeço também a minha namorada, em especial por sua compreensão, por me criticar e querer me fazer melhorar. Ela teve paciência para acompanhar meus ensaios e mesmo não sendo da área de exatas, acabou por entender e discutir comigo as idéias gerais de derivadas, integrais, controle, minimização e etc..

Aos professores e escolas aonde estudei e aprendi, aos caros colegas da USP e ao magnifico corpo docente desta instituição. Penso ter encontrado pessoas sábias e motivadoras, pessoas que me instigaram a conhecer mais. Em especial ao orientador, Prof. Dr. Paulo Sérgio, que me ensinou como apresentar uma idéia e me indicou quando algumas tarefas estavam bem encaminhadas, acabou por se tornar um amigo. 


\section{Agradecimentos}

A realização deste trabalho não seria possível sem a ajuda e conselhos de algumas pessoas, gostaria de agradecer a todos:

Ao Professor Walter Kaiser do Departamento de Engenharia de Energia e Automação Elétrica da Escola Politécnica da USP, pelas dicas e conselhos na usinagem e construção do pêndulo.

Aos professores e equipe do LAC, pelo custeio das peças, instruções de uso da placa de aquisição e laboratórios.

Aos amigos e colegas da FEI e da USP. Pessoas que deram sugestões e fizeram perguntas inusitadas, me fazendo assim olhar os problemas de uma ótica diferente. 


\section{Resumo}

Este trabalho versa sobre técnicas de estimação de derivadas de forma não assintótica conforme abordagem algébrica de Michel Fliess, e sua aplicação na determinação quase instantânea do estado interno de um sistema dinâmico, cria-se assim estimadores de estado que não se baseiam no observador de Luenberger.

No desenvolvimento do trabalho demonstramos algumas características destes estimadores e apresentamos uma contribuição teórica para viabilizar a implementação destes estimadores em sistemas de controle de tempo real. Posteriormente, um sistema mecânico de dinâmica não linear foi construído e permitiu ensaios em laboratório que atestam, através dos resultados experimentais encontrados, a funcionalidade deste tipo de estimador de estados.

PALAVRAS CHAVE: Controle (Teoria e sistemas de controle). Cálculo Operacional. Sistemas dinâmicos. Sistemas não Lineares. 


\section{Abstract}

This work is about derivative estimation technique based on a algebraic and non-asymptotically approach, as devised by Michel Fliess, applied on quasi-instantaneous determination of the internal state of a dynamical system, using state estimators that aren't based on the Luenberger observer.

Over this work we present some particularities of these estimators and a theoretical contribution that will able to implement these algebraic estimators in a real time control system. After that, a non-linear mechanical system was built to verify the functionality of these state estimators.

KEYWORDS: Control Theory. Operational Calculus. Dynamic Systems. Non-linear Systems. 


\section{Lista de Figuras}

2.1 Esquema de chaveamento entre dois estimadores. . . . . . . . . . . p.33

2.2 Estimando a primeira derivada de $y(t)=\sin (t) \ldots \ldots \ldots$ p. 34

2.3 Estimando a primeira derivada de $y(t)=\sin (t) \ldots \ldots \ldots \ldots$ p. 35

2.4 Estimando a primeira derivada de $y(t)=\sin (t)+\frac{\sin (100 t)}{1000}+\frac{\sin (10000 t)}{1000000} . \quad$ p. 36

2.5 Detalhe da estimativa da primeira derivada de $y(t)=\sin (t)+\frac{\sin (100 t)}{1000}+$

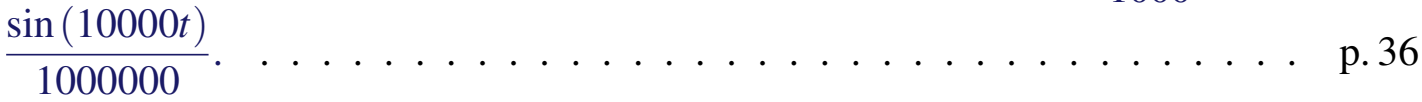

2.6 Detalhe da estimativa da primeira derivada de $y(t)=\sin (t)+\frac{\sin (100 t)}{1000}+$

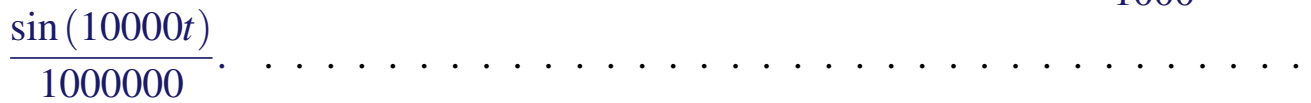

2.7 Estimando a primeira derivada de $y(t)=\sin (t)$ quando comprometido por

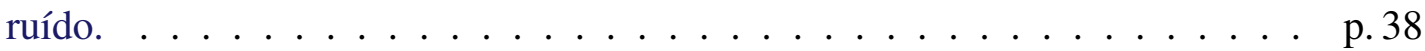

3.1 Resultado da integração numérica para diferentes passos de integração (de

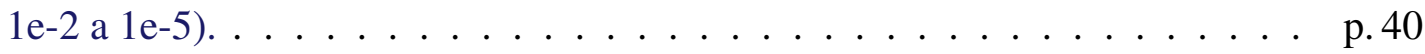

3.2 Detalhamento da convergência da integração numérica $\ldots \ldots \ldots$. . . . . p.41

3.3 Erro absoluto da integração numérica em função do passo de integração. . . . p.41

3.4 Estimador Algébrico sem ruído. . . . . . . . . . . . . . . . . . p. 44

3.5 Detalhe do Estimador Algébrico sem ruído. . . . . . . . . . . . . p. 45

3.6 Erro no Estimador Algébrico sem ruído. . . . . . . . . . . . . . p.45

3.7 Detalhe do erro no Estimador Algébrico sem ruído. $\ldots \ldots \ldots \ldots$. . . . . p. 46

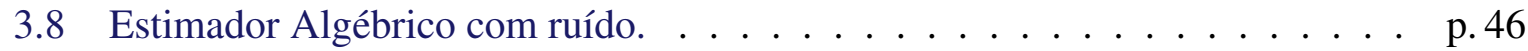

3.9 Detalhe do Estimador Algébrico com ruído. . . . . . . . . . . . . . p. 47

3.10 Erro no Estimador Algébrico com ruído. . . . . . . . . . . . . p.47

3.11 Detalhe do erro no Estimador Algébrico com ruído. . . . . . . . . . . p. 48 
3.12 Comportamento da saída. . . . . . . . . . . . . . . . p. 50

3.13 Comportamento dos estados internos . . . . . . . . . . . . p. 50

3.14 Comportamento do estimador. . . . . . . . . . . . . p.51

3.15 Comportamento do estimador na presença de ruído. . . . . . . . . . p.51

3.16 Comportamento da saída. . . . . . . . . . . . . . . . p.52

3.17 Comportamento do estimador. . . . . . . . . . . . . p. 52

3.18 Comportamento da saída quando o estimador é perturbado por ruído. . . . . . p.53

3.19 Comportamento do estimador quando perturbado por ruído. . . . . . . . . p.53

4.1 Visão geral do pêndulo. . . . . . . . . . . . . . . . p. 56

4.2 Ilustração das Coordenadas Utilizadas. . . . . . . . . . . . . . . p. 58

4.3 Gráfico de comportamento do modelo de atrito. . . . . . . . . . . . p. 63

4.4 Ensaio do modelo de atrito, força de $1 \mathrm{~N}$ aplicada ao bloco $\ldots \ldots$. . . . . . p. 64

4.5 Ensaio do modelo de atrito, força de $2 \mathrm{~N}$ aplicada ao bloco $\ldots \ldots$. . . . . p. 65

4.6 Ensaio do modelo de atrito, bloco com velocidade inicial. . . . . . . . . . p. 65

4.7 Gráfico de comportamento do modelo de atrito. . . . . . . . . . . . . . p.70

4.8 Gráfico de comportamento do modelo de atrito. . . . . . . . . . . . . . p.70

4.9 Resultado de ensaio, após filtragem. . . . . . . . . . . . p.71

4.10 Atrito cinético + viscoso. . . . . . . . . . . . . . . p. 72

4.11 Atrito cinético + viscoso $\ldots \ldots \ldots \ldots \ldots$ p. 72

4.12 Detalhe do Atrito cinético + viscoso. . . . . . . . . . . . p.73

4.13 Modelo e Ensaio . . . . . . . . . . . . . . . . . . p. 74

5.1 Simulação de Controle Linear . . . . . . . . . . . . . . . p. 76

5.2 Simulação de Controle Linear por observadores assintóticos de estado. . . . . p.77

5.3 Simulação de Controle Linear por observadores assintóticos de estado em presença de ruído aditivo. . . . . . . . . . . . . . p.78

5.4 Simulação de Controle Linear por observador algébrico $\ldots \ldots \ldots$. . . . . . p. 78 
5.5 Simulação de Controle Linear por observador algébrico na presença de ruído p.79

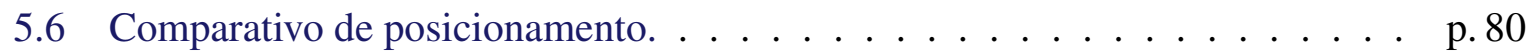

5.7 Diferença de posicionamento do braço em relação à realimentação de estados reais. . . . . . . . . . . . . . . . . p. 80

5.8 Comparativo de posicionamento em ambiente ruidoso. . . . . . . . . . p. 81

5.9 Diferença de posicionamento em relação à realimentação de estados reais, ambiente ruidoso. . . . . . . . . . . . . . . p. 81

5.10 Valores Estimados de $\phi \ldots \ldots \ldots \ldots \ldots \ldots$ p. . . . . . . . . . . . .

5.11 Diferença entre os valores estimados e o real valor de $\phi . \ldots \ldots$. . . . 82

5.12 Valores Estimados de $\phi \ldots \ldots \ldots \ldots \ldots$ p. . . . . . . . . . . . .

5.13 Diferença entre os valores estimados e o real valor de $\phi$, ambiente ruidoso. . . p. 83

5.14 Valores Estimados de $\dot{\phi} \ldots \ldots \ldots \ldots$. . . . . . . . . . . . 84

5.15 Diferença entre os valores estimados e o valor real de $\dot{\phi} . \quad \ldots \ldots$. . . . . p. 84

5.16 Valores Estimados de $\dot{\phi}$, ambiente ruidoso. . . . . . . . . . . . p. 85

5.17 Diferença entre os valores estimados e o valor real de $\dot{\phi}$, ambiente ruidoso. . . p. 85

5.18 Posicionamento do braço no ensaio em bancada. . . . . . . . . . . . . p. 86

5.19 Comparativo entre os observadores de estado. . . . . . . . . . . . . p.87

5.20 Comparativo entre os observadores de estado. . . . . . . . . . . . . . p.87

5.21 Comparativo entre os observadores de estado. . . . . . . . . . p.88

5.22 Comparativo entre os observadores de estado. . . . . . . . . . . . . p. 88

5.23 Comparativo entre os esforços de controle. . . . . . . . . . . . . p. 89

B.1 Esquema fundamental do estimador. . . . . . . . . . . . . . . p.99

B.2 Esquema detalhado do estimador de derivadas . . . . . . . . . . p. 100

B.3 Fluxograma do algorítimo. . . . . . . . . . . . . p. 105

C.1 Modelo Global . . . . . . . . . . . . . . . . . . . . . . . p. 119

C.2 Modelo Completo do Pêndulo. . . . . . . . . . . . . . . . . p. 120

C.3 Modelo Idealizado do Pêndulo. . . . . . . . . . . . . . . . . . p. 120 
C.4 Modelo do Motor. . . . . . . . . . . . . . . . . . . . p. 121

C.5 Modelo do Observador Linear. . . . . . . . . . . . . . . . . . . . . p. 121

C.6 Modelo do Observador Algébrico. . . . . . . . . . . . . . . . p. 122

C.7 Modelo de Atrito. . . . . . . . . . . . . . . . . . . . . p. 122

C.8 Circuito elétrico do leitor do encoder . . . . . . . . . . . . . p. 126

C.9 Circuito elétrico do display de leds . . . . . . . . . . . . . . . p. 127

C.10 Circuito elétrico do estágio de potência . . . . . . . . . . . . p. 128

C.11 Desenho da Barra . . . . . . . . . . . . . . . . . . . . . p. 129

C.12 Chapa de Fixação do Encoder. . . . . . . . . . . . . . . . . p. 130

C.13 Contra Peso. . . . . . . . . . . . . . . . . . p. 131

C.14 Eixo. . . . . . . . . . . . . . . . . . . p. 132

C.15 Flange do Disco. . . . . . . . . . . . . . . . p. 133

C.16 Lateral 1. . . . . . . . . . . . . . . . . . . . . p. 134

C.17 Lateral 2. . . . . . . . . . . . . . . . . . . p. 135

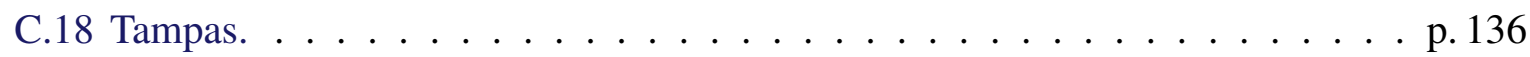

C.19 Pilar. . . . . . . . . . . . . . . . . . . p. 137 


\section{Lista de Tabelas}

2.1 Constantes relativas ao estimador algébrico $\ldots \ldots \ldots \ldots$. . . . . . . . . . 29

2.2 Constantes para estimador de ordem $3 \ldots \ldots \ldots \ldots$. . . . . . . . . . . . .

4.1 Coordenadas Utilizadas. . . . . . . . . . . . . . . . p.57

4.2 Simbologia das grandezas físicas. . . . . . . . . . . p. 58

4.3 Simbologia dos componentes do sistema. . . . . . . . . . . . . . p. 59

4.4 Propriedades físicas do sistema mecânico . . . . . . . . . . . . p. 68

B.1 Parâmetros do Estimador em tempo discreto. . . . . . . . . . . . . p. 101

B.2 Coeficientes do estimador. . . . . . . . . . . . . p. 102 


\section{Sumário}

Tabela de Símbolos.

p. 15

1 Introdução $\quad$ p. 17

1.1 Objetivo deste trabalho $\ldots \ldots \ldots \ldots \ldots \ldots$ p. 18

1.2 Estrutura do trabalho $\ldots \ldots \ldots \ldots \ldots \ldots \ldots$

2 Sobre a teoria de observadores rápidos $\quad$ p. 20

2.1 Introdução sobre a teoria de observadores convencional . . . . . . . . . p. 21

2.2 Introdução sobre a teoria de observadores rápidos . . . . . . . . . . . . p. 22

2.3 Representação via filtros variantes no tempo . . . . . . . . . . . . . . p. 22

2.3.1 Exemplo algébrico de ordem baixa . . . . . . . . . . . . p.30

2.4 Particularidades da teoria de estimadores rápidos $\ldots \ldots \ldots \ldots$. . . . . . 32

2.5 Uso de dois estimadores em paralelo . . . . . . . . . . . . p.33

2.6 Uso de filtragem adicional $\ldots \ldots \ldots \ldots \ldots \ldots$ p. . . . . . . . . . . . . .

2.7 Simulações . . . . . . . . . . . . . . . . . . . . . . p. . 34

3 Implementação em tempo discreto $\quad$ p. 39

3.1 Dificuldades relativas à implementação . . . . . . . . . . . . . . . . p. 40

3.2 Abordagem via aproximação do sinal amostrado por segmentos de polinômios p. 42

3.2.1 Comparando a técnica descrita com a integração numérica de um sistema caótico . . . . . . . . . . . . . . p. 43

3.3 Breve aplicação em controle . . . . . . . . . . . . . . . . . p.48

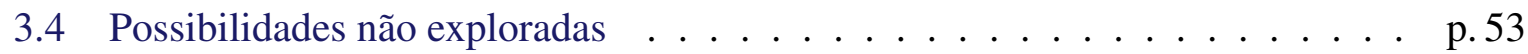


4 Sistema Mecânico $\quad$ p. 55

4.1 Descrição do sistema . . . . . . . . . . . . . . . . p. 56

4.2 Modelagem do sistema $\ldots \ldots \ldots \ldots \ldots \ldots$ p. . . . . . . . . . . . . . . .

4.2.1 Definição da simbologia adotada . . . . . . . . . . . . p. p. . .

4.2.2 Modelagem do sistema através do formalismo Lagrangiano $\quad \ldots . . \quad$ p.59

4.2.3 Modelagem do atrito . . . . . . . . . . . . . p. 63

4.2.4 Motor como transdutor de torque ............. p. 65

4.2.5 Modelo linearizado . . . . . . . . . . . . . p. 66

4.2.6 Parâmetros numéricos do modelo . . . . . . . . . . . . p. 66

5 Controle do Pêndulo por transmissão de torque p.75

5.1 Realimentação linear de estados . . . . . . . . . . . . . . p. 76

5.2 Observador assintótico para o sistema linearizado . . . . . . . . . . p.77

5.3 Controle com estimador algébrico $\ldots \ldots \ldots \ldots \ldots \ldots$ p. 78

5.4 Comparações entre os observadores . . . . . . . . . . . . . . p. 79

5.4.1 Posicionamento do braço do pêndulo. . . . . . . . . . . . p. 79

5.4.2 Estimador de estados $\phi \ldots \ldots \ldots \ldots \ldots$ p. 81

5.4.3 Estimador de estados $\dot{\phi} \ldots \ldots \ldots \ldots$ p. 83

5.5 Ensaio de Bancada com o pêndulo por transferência de torque $\ldots \ldots$. . . p. 86

5.5.1 Comparativo do resultado de controle . . . . . . . . . . p. 86

5.5.2 Comparativo entre os observadores ........... . . p. 86

5.5.3 Comparativo entre os esforços de controle . . . . . . . . . . . p. 89

5.5.4 Considerações finais sobre o ensaio realizado . . . . . . . . . . . p. 89

6 Conclusões e Trabalhos Futuros p.91

$\begin{array}{ll}\text { Referências Bibliográficas } & \text { p.93 }\end{array}$ 
$\begin{array}{lr}\text { Anexo A - Demonstrações } & \text { p.94 }\end{array}$

A.1 Demonstração do resultado sobre integrais de polinômios . . . . . . . . . . . p.94

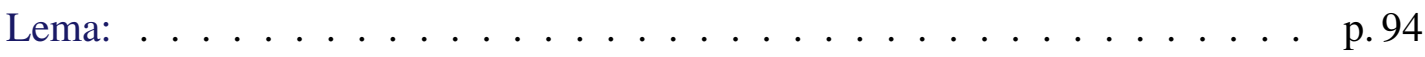

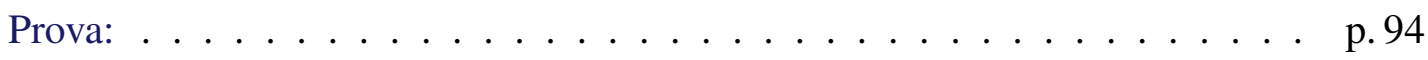

A.2 Equação do filtro $\mathrm{Z}$ em tempo discreto $\ldots \ldots \ldots \ldots$ p. . . . . . . . . . . .

$\begin{array}{lr}\text { Anexo B - Resumo de Implementação } & \text { p. } 99\end{array}$

B.1 Definição dos parâmetros do sistema . . . . . . . . . . . . . . . . p. 100

B.2 Pré cálculo dos coeficientes . . . . . . . . . . . . . . . . . p. 102

B.2.1 Cálculo da matriz de aproximação polinomial para cada instante possível de amostragem . . . . . . . . . . . . . . . p. 102

B.2.2 Cálculo das constantes do estimador . . . . . . . . . . . . . p. 102

B.3 Construção do estimador . . . . . . . . . . . . . . . p. 103

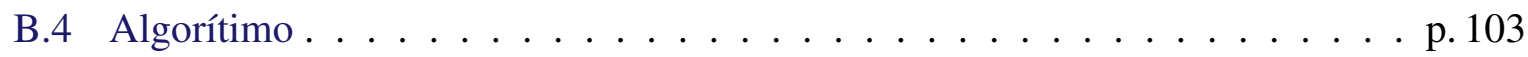

B.4.1 Ambos os contadores de tempo ( $t a$ e $t b)$ serão incrementados de $\Delta_{t} \quad$. p. 103

B.4.2 Deslocar amostras em $y_{b u f f e r}$ e inserir amostra atual na primeira posiçãop. 103

B.4.3 Determinar os coeficientes $\alpha$ para o filtro $Z a \ldots$. . . . . . . p. 104

B.4.4 Determinar a evolução dos estágios do filtro $\mathrm{Za}$. . . . . . . . . . . p. 104

B.4.5 Determinar os coeficientes $\alpha$ para o filtro $Z b \ldots \ldots$. . . . . 104

B.4.6 Determinar a evolução dos estágios do filtro $Z b$. . . . . . . . . . p. 104

B.4.7 Escolher os estágios do filtro cujo tempo relativo ( $t a$ ou $t b)$ seja maior e usar estes valores no cálculo das estimativas das derivadas . . . . . p. 104

B.4.8 Se o contador de tempo ta tiver valor igual a metade do valor $t_{\text {reset }}$, reinicializar o vetor $Z b$ e o contador $t b$. Se isto não ocorrer, então verificar se o contador $t b$ tem valor igual ou superior a metade do valor $t_{\text {reset }}$ e neste caso reinicializar o vetor $Z a$ e o contador $t a$. Esta política implementa o procedimento de reinicialização alternada ilustrado em

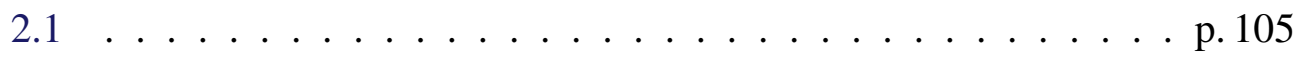

B.4.9 Prosseguir com a próxima amostra . . . . . . . . . p. 105 
$\begin{array}{ll}\text { Anexo C - Ferramentas utilizadas } & \text { p. } 106\end{array}$

C.1 Introdução . . . . . . . . . . . . . . . . . . p. 106

C.2 Programas para ambiente MATLAB . . . . . . . . . . p. 106

C.2.1 Programa Makkar_Solve.m . . . . . . . . . . . p. 106

C.2.2 Programa NAO_disc_sfunc.m . . . . . . . . . . . p. 108

C.2.3 Programa SGDerivation.m . . . . . . . . . . . p. 115

C.2.4 Programa Parametros.m . . . . . . . . . . . p. 116

C.2.5 Programa IdentificaAtritoBarra.m . . . . . . . . . . . p. 117

C.3 Modelos para SIMULINK. . . . . . . . . . . . . . . . p. 119

C.3.1 Modelo Global . . . . . . . . . . . . . . . . . p. 119

C.3.2 Modelo completo do Pêndulo . . . . . . . . . . . . p. 120

C.3.3 Modelo idealizado do Pêndulo . . . . . . . . . . . . . p. 120

C.3.4 Modelo do Motor . . . . . . . . . . . . . . . . p. 121

C.3.5 Modelo do Observador linear . . . . . . . . . . . . . . p. 121

C.3.6 Modelo do Observador algébrico . . . . . . . . . . . . . . . . . p. 122

C.3.7 Modelo gerador de atrito . . . . . . . . . . . . . . . . p. 122

C.4 Programa para microcontrolador PIC . . . . . . . . . . . . . . p. 122

C.5 Diagrama elétrico dos dispositivos de interface . . . . . . . . . p. 126

C.5.1 Circuito de leitura do encoder . . . . . . . . . . . . p. 126

C.5.2 Circuito de saída de potência . . . . . . . . . . . . . p. 128

C.6 Desenho das peças usinadas na construção do pêndulo . . . . . . . . . . . . p. 129

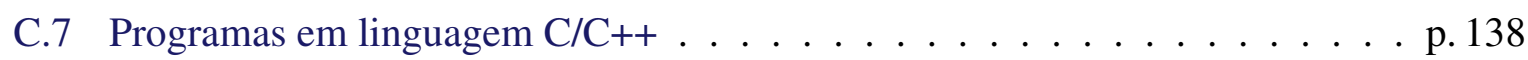

C.7.1 Interface para o subsistema Xenomai . . . . . . . . . . p. 138

C.7.2 Implementação discreta do observador algébrico . . . . . . . . . . p. 146

C.7.3 Rotinas para criar arquivos mat . . . . . . . . . . . p. 164

C.7.4 Driver para acesso à placa de aquisição LYNX12/36 . . . . . . . . . . p. 169 


\section{Tabela de Símbolos.}

\begin{tabular}{|c|c|}
\hline Símbolo & Descrição \\
\hline$y(t)$ & Sinal cujas derivadas serão estimadas. \\
\hline$k$ & Ordem da expansão em serie de Taylor usada no estimador algébrico. \\
\hline$a(k, \delta, l)$ & Coeficiente utilizado no desenvolvimento das equações do estimador algébrico. \\
\hline$b(k, \delta, m)$ & $\begin{array}{l}\text { Coeficiente utilizado no desenvolvimento e resultado final das equações do } \\
\text { estimador algébrico. Ver tabela } 2.1\end{array}$ \\
\hline$D(k, i, j)$ & $\begin{array}{l}\text { Coeficiente utilizado no desenvolvimento e resultado final das equações do } \\
\text { estimador algébrico.Ver tabela } 2.1\end{array}$ \\
\hline$E(k, i)$ & $\begin{array}{l}\text { Coeficiente utilizado no desenvolvimento e resultado final das equações do } \\
\text { estimador algébrico.Ver tabela } 2.1\end{array}$ \\
\hline$z_{i}(k, t)$ & Estágio $i$ da construção como filtro variante no tempo. \\
\hline$p$ & $\begin{array}{l}\text { Grau do polinômio que aproxima as amostras do sinal de entrada na } \\
\text { implementação em tempo discreto. }\end{array}$ \\
\hline$t_{0}$ & $\begin{array}{l}\text { Valor numérico do tempo em que ocorre a amostra atual do sinal discretizado. } \\
\text { Este tempo não é absoluto, é relativo à última reinicialização do estimador. }\end{array}$ \\
\hline$t_{i}$ & $\begin{array}{l}\text { Da mesma maneira que } t_{0} \text {, se referindo ao tempo em } i \text { amostras antes da atual e } \\
\text { relativo à última reinicialização do estimador. }\end{array}$ \\
\hline$\Delta_{t}$ & Intervalo de amostragem. \\
\hline$\alpha_{i}$ & $\begin{array}{l}\text { Coeficientes } i \text { do polinômio de grau } p \text { que aproxima as últimas } p+1 \text { amostras } \\
\text { do sinal na implementação em tempo discreto. }\end{array}$ \\
\hline $\mathbb{A}\left(t_{0}\right)$ & $\begin{array}{l}\text { Matriz variante no tempo que define os coeficientes } \alpha_{i} \text { como uma combinação } \\
\text { linear das últimas } p+1 \text { amostras do sinal de entrada. }\end{array}$ \\
\hline$\phi$ & $\begin{array}{l}\text { Ângulo formado entre a barra e o plano vertical, nulo quando em equilíbrio não } \\
\text { trivial, para } 0<\eta<2 \pi \text {. }\end{array}$ \\
\hline$\theta$ & Ângulo formado entre um ponto de referência no disco e a barra. \\
\hline$\eta$ & $\begin{array}{l}\text { Ângulo formado entre o plano da barra e o plano horizontal. Constante durante } \\
\text { os ensaios. }\end{array}$ \\
\hline$h$ & Altura. Ver tabelas 4.2 e 4.3 . \\
\hline$M$ & Massa. Ver tabelas 4.2 e 4.3. \\
\hline
\end{tabular}




\begin{tabular}{|l|l|}
\hline \hline Símbolo & Descrição \\
\hline \hline$L$ & Comprimento. Ver tabelas 4.2 e 4.3. \\
\hline$\tau$ & Torque. Ver tabelas 4.2 e 4.3. \\
\hline$J$ & Momento de Inercia. Ver tabelas 4.2 e 4.3. \\
\hline$\tau_{a t,}$ & Torque de Atrito. Ver tabelas 4.2 e 4.3. \\
\hline$M O T$ & Motor. Ver tabelas 4.2 e 4.3. \\
\hline$D I S$ & Disco. Ver tabelas 4.2 e 4.3. \\
\hline$C I L$ & Cilindro de Contra Peso. Ver tabelas 4.2 e 4.3. \\
\hline$B A R$ & Barra em sua totalidade. Ver tabelas 4.2 e 4.3. \\
\hline$B A R 1$ & Trecho da barra que sustenta o motor e disco. Ver tabelas 4.2 e 4.3. \\
\hline$B A R 2$ & Trecho da barra que sustenta o contra peso. Ver tabelas 4.2 e 4.3. \\
\hline $\begin{array}{l}\mathbb{C}_{1}, \mathbb{C}_{2}, \\
\mathbb{C}_{3}\end{array}$ & Constantes mecânicas do pêndulo. \\
\hline$\gamma_{i}$ & Coeficientes do modelo de atrito utilizado nas simulações. \\
\hline$K_{\tau, M O T}$ & $\begin{array}{l}\text { Constante de proporcionalidade entre torque e tensão de armadura no modelo } \\
\text { simplificado do motor operando em baixas velocidades. }\end{array}$ \\
\hline$K_{M O T}$ & Constante de torque e de força contra eletromotriz do motor. \\
\hline$V a_{M O T}$ & Tensão de armadura do motor. \\
\hline
\end{tabular}




\section{$1 \quad$ Introdução}


A teoria desenvolvida em recentes trabalhos dos autores de [1, 2] abre a possibilidade de se construir estimadores de estado rápidos e ao mesmo tempo capazes de rejeitar ruídos. Tais estimadores se valem de métodos algébricos para estimar as derivadas de um sinal a partir de combinações de suas integrais no tempo. Destaca-se em [1] a visualização destes estimadores algébricos como filtros analógicos variantes no tempo, e em [2] propõe-se o uso de dois estimadores rodando em paralelo de maneira a aumentar a precisão e robustez das derivadas estimadas. O trabalho desenvolvido em [3] demonstra como implementar tais filtros em computadores digitais com taxas de amostragem relativamente baixas e sem a necessidade de elevado poder computacional, viabilizando o uso desta teoria em sistemas de controle em tempo real.

\subsection{Objetivo deste trabalho}

O principal objetivo desta pesquisa é utilizar estes métodos de estimação para efetuar a observação e controle de alguns sistemas dinâmicos, e estabelecer comparações com observadores tradicionais. Um sistema mecânico, pêndulo invertido por transmissão de torque, foi construído e os resultados dos ensaios de laboratório serão discutidos e utilizados para complementar estas comparações com dados práticos.

\subsection{Estrutura do trabalho}

Este trabalho está dividido em seis capítulos, iniciando por esta introdução e finalizando, no capítulo 6, com uma discussão das conclusões e aprendizados obtidos e sugestões para pesquisas futuras.

O capítulo 2 introduz a teoria de observadores rápidos, transcrevendo alguns resultados de [1], discute suas características e apresenta algumas simulações. Este capítulo constitui uma pequena introdução à teoria criada por Fliess e co-autores, limitando-se a demonstrar os resultados mais fundamentais para continuar esta dissertação.

No capítulo 3 tratamos da implementação desta teoria em ambiente computacional e sobre sua aplicação em sistemas de controle de tempo real, introduzindo uma técnica que encontramos para viabilizar a implementação destes estimadores. Finalizando o capítulo 3 apresentamos uma pequena aplicação de controle utilizando o estimador algébrico implementado em tempo discreto.

O capítulo 4 versa sobre o sistema mecânico construído, descrevendo e deduzindo suas equações de movimento e introduzindo um modelo de atrito. Após ensaios preliminares, alguns 
valores numéricos são estimados.

O controle do sistema é discutido no capítulo 5, onde apresentamos os resultados experimentais encontrados.

A seção de apêndices apresenta algumas demonstrações e detalha as ferramentas, programas e diagramas de simulação. 


\title{
2 Sobre a teoria de observadores rápidos
}

\author{
“... considerando que quaisquer pensamentos que nos ocorrem quando \\ estamos acordados nos podem também ocorrer enquanto dormimos, \\ sem que exista nenhum, nesse caso, que seja correto,
}

decidi fazer de conta que todas as coisas que até então haviam entrado no meu espírito não eram mais corretas do que as ilusões de meus sonhos. Porém, logo em seguida, percebi que, ao mesmo tempo que eu queria pensar que tudo era falso, fazia- se necessário que eu, que pensava, fosse alguma coisa.

E, ao notar que esta verdade: eu penso, logo existo, era tão sólida e tão correta que as mais extravagantes suposições dos céticos não seriam capazes de lhe causar abalo, julguei que podia considerá-la, sem escrúpulo algum, o primeiro princípio da filosofia que eu procurava." (René Descartes - O Discurso do Método)

Neste capítulo são apresentados os conceitos nos quais se baseia o presente trabalho. Uma breve apresentação da teoria de observadores assintóticos é apresentada, transcrição e tradução livre de alguns trechos de [1].

As características especiais destes estimadores são discutidas e embasadas em simulações e exemplos. 


\subsection{Introdução sobre a teoria de observadores convencional}

A teoria de controle moderno se baseia na realimentação de estados para alocar os pólos controláveis de um sistema dinâmico linear. Porem estes estados muitas vezes não se encontram acessíveis ao projetista de controle e é necessário que sejam estimados. O observador de Luenberger, devidamente projetado para um dado sistema dinâmico, permite a estimação assintótica dos estados internos tomando informações das entradas e saídas deste sistema. Este observador é construído a partir de um modelo do sistema dinâmico onde se injetam as entradas do sistema real.

Obviamente devido às características assintóticas deste observador de estados, ou seja, o estado é estimado com um erro que tende assintoticamente a zero, a realimentação dos estados observados não produz um efeito idêntico à realimentação dos estados reais. Isto não é um grande problema em sistemas lineares, pois devido ao teorema da separação, se o observador e a lei de realimentação de estados forem estáveis, a realimentação dos estados observados será estável e terá os pólos do observador e da lei de controle. Já quando tratamos de sistemas não lineares, o teorema da separação não é normalmente válido e, em geral, não se pode atestar a estabilidade de uma realimentação de estados [4].

A teoria nos mostra que um sistema dinâmico linear que atenda as características de controlabilidade pode ter seus pólos livremente alocados no plano complexo pela aplicação em sua entrada de uma adequada combinação linear dos seus estados internos. Desta maneira, basta se determinar os pesos para a adequada realimentação de cada estado interno e poderemos modificar com bastante liberdade o comportamento de um sistema dinâmico [5].

Tirar proveito desta característica (controlabilidade) é muito interessante, mas normalmente não se tem, em um sistema real, acesso a todos os estados internos. Neste momento, é necessário que o sistema seja também observável, o que implica que o estado interno pode ser deduzido quando se conhece a entrada aplicada e a saída obtida [5].

A teoria nos mostra também, que se um sistema linear, autônomo e invariante no tempo é observável, então seu estado interno pode ser instantaneamente deduzido pela adequada combinação linear da saída e um número finito de suas derivadas no tempo. Obter derivadas a partir de um sinal real e sujeito a ruídos e imprecisões de leitura não é tarefa simples, e por este motivo, a teoria moderna de controle utiliza observadores assintóticos, ou seja, sistemas dinâmicos em que se injetam as entradas e saídas do sistema observado e que geram estimativas dos estados internos. Estas estimativas tendem assintoticamente ao estado real, por este motivo são chamados de observadores assintóticos. 


\subsection{Introdução sobre a teoria de observadores rápidos}

Utilizando-se de métodos algébricos e cálculo operacional, é possível construir um estimador rápido de derivadas que seja ao mesmo tempo causal e robusto com relação a ruídos. Esta teoria é desenvolvida nos trabalhos de Fliess e co-autores,[6, 7, 1, 2, 8], para uma aproximação polinomial em série de Taylor, truncada em uma ordem finita $k$. Demonstra-se que as derivadas de ordem 0 até $k-1$ desta aproximação polinomial podem ser obtidas pela combinação de um número finito de suas integrais. Posteriormente, a aproximação polinomial é substituída pelo sinal real e obtém-se um estimador que é, em teoria, instantâneo e capaz de rejeitar ruídos.

\subsection{Representação via filtros variantes no tempo}

Em [1] a técnica descrita é desenvolvida de maneira a chegar em uma composição de fácil interpretação para se estimar as derivadas de um sinal analógico. Devido à grande relevância para o presente trabalho, uma pequena parte deste material será brevemente transcrita, com modificações mínimas, nas linhas a seguir:

Seja um sinal analógico $y(t)$, aproximado em torno de $t=t_{i}$ por uma expansão em série de Taylor truncada em uma ordem $k$ suficiente.

$$
\tilde{y}(t)=\sum_{j=1}^{k} \frac{1}{(j-1) !} y^{(j-1)}\left(t_{i}\right)\left(t-t_{i}\right)^{j-1} .
$$

Esta série pode ser vista como um encadeamento de integradores onde $\tilde{y}^{(k)}(t) \equiv 0$ e as condições iniciais são $\tilde{y}^{(j-1)}\left(t_{i}\right)=y^{(j-1)}\left(t_{i}\right)$. Fazendo $\tau=t-t_{i}$ :

$$
\tilde{y}^{(k)}(\tau)=0, \tilde{y}^{(j-1)}(\tau)=\tilde{y}^{(j-1)}\left(t_{i}\right), j=1,2, \ldots, k
$$

No campo do cálculo operacional ${ }^{1}$, pode-se escrever [9]:

$$
s^{k} \tilde{y}(s)-\sum_{j=1}^{k} s^{k-j} y^{(j-1)}\left(t_{i}\right)=0 .
$$

Derivando $k$ vezes em relação ao operador $s$

\footnotetext{
${ }^{1}$ Aqui cálculo operacional designa essencialmente as propriedades da transformada de Laplace.
} 


$$
\frac{d^{k}}{d s^{k}}\left(s^{k} \tilde{y}(s)\right)=0
$$

equação que é livre de qualquer condição inicial do sinal.

Multiplicando por $s^{-j} j=k-1, k-2, \ldots, k-d$ resultam $d$ equações

$$
0=s^{-j} \frac{d^{k}}{d s^{k}}\left(s^{k} \tilde{y}(s)\right), j=k-1, k-2, \ldots, k-d
$$

o que constitui um sistema triangular de equações lineares para calcular a estimativa das derivadas no tempo de $y(t)$ até a ordem $d \leq k-1$. Isto pode ser observado através da fórmula de Leibniz:

$$
\frac{d^{k}}{d s^{k}}(x(s) y(s))=\sum_{i=0}^{k}\left(\begin{array}{c}
k \\
i
\end{array}\right) \frac{d^{k-i}}{d s^{k-i}} x(s) \frac{d^{i}}{d s^{i}} y(s)
$$

fazendo:

$$
\frac{d^{k-i}}{d s^{k-i}} x(s)=\frac{d^{k-i}}{d s^{k-i}} s^{k}=\frac{k !}{i !} s^{i}
$$

Aplicando este resultado na equação (2.5) vem:

$$
s^{-j} \frac{d^{k}}{d s^{k}}\left(s^{k} \tilde{y}(s)\right)=\sum_{i=0}^{k} \frac{(k !)^{2}}{(k-i) !(i !)^{2}} s^{i-j} \frac{d^{i}}{d s^{i}} \tilde{y}(s)=\sum_{i=0}^{k}\left(\begin{array}{c}
k \\
i
\end{array}\right)^{2}(k-i) ! s^{i-j} \frac{d^{i}}{d s^{i}} \tilde{y}(s)
$$

para $j=k-1, k-2, \ldots, k-d$. Reescrevendo (2.8) em dois somatórios (aqui cabe chamar a atenção para os grupos de somatórios, o primeiro diz respeito às derivadas de $\tilde{y}(t)$ enquanto que o segundo opera em suas integrais)

$$
0=\sum_{i=j}^{k}\left(\begin{array}{c}
k \\
i
\end{array}\right)^{2}(k-i) ! s^{i-j} \frac{d^{i}}{d s^{i}} \tilde{y}(s)+\sum_{i=0}^{j-1}\left(\begin{array}{c}
k \\
i
\end{array}\right)^{2}(k-i) ! s^{i-j} \frac{d^{i}}{d s^{i}} \tilde{y}(s) .
$$

Através da transformação usual para retornar ao domínio do tempo, obtém-se $d$ equações:

$$
0=\sum_{i=j}^{k}\left(\begin{array}{c}
k \\
i
\end{array}\right)^{2}(k-i) ! \frac{d^{i-j}}{d t^{i-j}}\left((-t)^{i} \tilde{y}(t)\right)+\sum_{i=0}^{j-1}\left(\begin{array}{c}
k \\
i
\end{array}\right)^{2}(k-i) ! \int^{(j-i)}(-t)^{i} \tilde{y}(t)
$$

aqui, para simplificar a escrita, adotou-se para as integrais a seguinte notação: 


$$
\int^{(j-i)}(-t)^{i} \tilde{y}(t) \triangleq \int_{0}^{t} \int_{0}^{\sigma_{1}} \cdots \int_{0}^{\sigma_{m-1}}\left(\sigma_{m}\right)^{i} \tilde{y}\left(\sigma_{m}\right) d \sigma_{m} d \sigma_{m-1} \cdots d \sigma_{1}
$$

Aplicando a fórmula de Leibniz na equação (2.10), isola-se $\tilde{y}(t)$ e suas derivadas no tempo $\tilde{y}^{(1)}(t), \cdots, \tilde{y}^{(k-d)}(t)$

$$
\begin{aligned}
0 & =\sum_{i=j}^{k}\left(\begin{array}{c}
k \\
i
\end{array}\right)^{2}(k-i) !(-1)^{i} \sum_{l=0}^{i-j}\left(\begin{array}{c}
i-j \\
l
\end{array}\right) \frac{i !}{(j+l) !} t^{j+l} \tilde{y}^{(l)}(t)+\sum_{i=0}^{j-1}\left(\begin{array}{c}
k \\
i
\end{array}\right)^{2}(k-i) !(-1)^{i} \int^{(j-i)} t^{i} \tilde{y}(t) \\
& =\sum_{i=j}^{k} \sum_{l=0}^{i-j}\left(\begin{array}{c}
k \\
i
\end{array}\right)\left(\begin{array}{c}
i-j \\
l
\end{array}\right) \frac{k !(-1)^{i}}{(j+l) !} t^{j+l} \tilde{y}^{(l)}(t)+\sum_{i=0}^{j-1}\left(\begin{array}{c}
k \\
i
\end{array}\right)^{2}(k-i) !(-1)^{i} \int^{(j-i)} t^{i} \tilde{y}(t) .
\end{aligned}
$$

Definindo-se o índice da maior derivada presente na equação (2.12), $\delta=k-j$, fazendo $n=i+\delta-k$ e introduzindo um contador de integração $m=k-\delta-i$, escreve-se:

$$
0=\underbrace{\sum_{n=0}^{\delta} \sum_{l=0}^{n}\left(\begin{array}{c}
k \\
\delta-n
\end{array}\right)\left(\begin{array}{l}
n \\
l
\end{array}\right) \frac{k !(-1)^{n+k-\delta}}{(k+l-\delta) !} t^{k+l-\delta} \tilde{y}^{(l)}(t)}_{\alpha(k, \delta, t)}+\underbrace{\sum_{m=1}^{k-\delta}\left(\begin{array}{c}
k \\
m+\delta
\end{array}\right)^{2}(m+\delta) !(-1)^{k-\delta-m} \int^{(m)} t^{k-m-\delta} \tilde{y}(t)}_{\beta(k, \delta, t)} .
$$

Note-se que a estrutura triangular de soma implica em:

$$
\sum_{n=0}^{\delta} \sum_{l=0}^{n} \cdots=\sum_{l=0}^{\delta} \sum_{n=l}^{\delta} \cdots
$$

e podemos então escrever:

$$
\begin{aligned}
\alpha(k, \delta, t) & =\sum_{l=0}^{\delta} \frac{k !(-1)^{k-\delta}}{(k+l-\delta) !}\left(\sum_{n=l}^{\delta}\left(\begin{array}{c}
k \\
\delta-n
\end{array}\right)\left(\begin{array}{l}
n \\
l
\end{array}\right)(-1)^{n}\right) t^{k+l-\delta} \tilde{y}^{(l)}(t) \\
& =\sum_{l=0}^{\delta} \frac{k !(-1)^{k-\delta}}{(k+l-\delta) !}\left(\frac{(k-l-1) !(-1)^{l}}{(k-\delta-1) !(\delta-l) !}\right) t^{k+l-\delta} \tilde{y}^{(l)}(t) \\
& =\sum_{l=0}^{\delta-1}\left(\begin{array}{c}
k \\
\delta-l
\end{array}\right) \frac{(k-l-1) !}{(k-\delta-1) !}(-1)^{l-\delta+1} t^{k+l-\delta} \tilde{y}^{(l)}(t)+(-1)^{k} t^{k} \tilde{y}^{(\delta)}(t) .
\end{aligned}
$$

Combinando este resultado com (2.13) obtém-se a seguinte fórmula recursiva nas derivadas. 


$$
\begin{aligned}
\tilde{y}^{(\delta)}(t) & =\sum_{l=0}^{\delta-1} \underbrace{\left(\begin{array}{c}
k \\
\delta-l
\end{array}\right) \frac{(k-l-1) !}{(k-\delta-1) !}(-1)^{l-\delta+1}}_{a(k, \delta, l)} \frac{1}{t^{\delta-l}} \tilde{y}^{(l)}(t) \\
& +\sum_{m=1}^{k-\delta} \underbrace{\left(\begin{array}{c}
k \\
m+\delta
\end{array}\right)^{2}(-1)^{1-m-\delta}(m+\delta) !}_{b(k, \delta, m)} \frac{1}{t^{k}} \int^{(m)} t^{k-m-\delta} \tilde{y}(t) .
\end{aligned}
$$

Para simplificar a visualização, isolamos dois blocos da equação (2.16) e definimos dois parâmetros, chamados de $a(k, \delta, l)$ e $b(k, \delta, m)$ como:

$$
\begin{aligned}
a(k, \delta, l) & =\left(\begin{array}{c}
k \\
\delta-l
\end{array}\right) \frac{(k-l-1) !}{(k-\delta-1) !}(-1)^{l-\delta+1} \\
b(k, \delta, m) & =\left(\begin{array}{c}
k \\
m+\delta
\end{array}\right)^{2}(-1)^{1-m-\delta}(m+\delta) !
\end{aligned}
$$

A equação (2.16) permite determinar as derivadas de ordem $\delta=1, \ldots, d \leq k-1$ do sinal $\tilde{y}(t)$. Como as derivadas $\tilde{y}^{(\delta)}$ dependem de todas as derivadas de ordem inferior $-\tilde{y}^{(\delta-1)}, \tilde{y}^{(\delta-2)}$, e assim por diante - esta equação resulta em um sistema triangular de equações. Como consequiência, as estimativas das derivadas reais se obtém apenas através de integrais de $\tilde{y}(t)$ ou, como este sinal pode ser medido, substituindo $\tilde{y}(t)$ pelo valor medido de $y(t)$.

O sistema de equações lineares descrito em (2.16) pode ser entendido como um filtro variante no tempo. Para ilustrar esta perspectiva, vamos definir: 


$$
\begin{aligned}
& \bar{y}^{(d)}(t)=\left(\begin{array}{c}
\tilde{y}^{(1)}(t) \\
\tilde{y}^{(2)}(t) \\
\vdots \\
\tilde{y}^{(d)}(t)
\end{array}\right) \\
& A(k, t)=\left(\begin{array}{ccccc}
t^{k} & 0 & 0 & 0 & 0 \\
-a(k, 2,1) t^{k-1} & t^{k} & 0 & 0 & 0 \\
-a(k, 3,1) t^{k-2} & -a(k, 3,2) t^{k-1} & t^{k} & 0 & 0 \\
\vdots & \vdots & \vdots & \ddots & 0 \\
-a(k, d, 1) t^{k-d+1} & -a(k, d, 2) t^{k-d+2} & -a(k, d, 3) t^{k-d+3} & \cdots & t^{k}
\end{array}\right) \\
& x(k, t)=\left(\begin{array}{c}
a(k, 1,0) t^{k-1} \\
a(k, 2,0) t^{k-2} \\
a(k, 3,0) t^{k-3} \\
\vdots \\
a(k, d, 0) t^{k-d}
\end{array}\right) y(t)+\left(\begin{array}{c}
\sum_{m=1}^{k-1} b(k, 1, m) \int t^{(m)} t^{k-m-1} y(t) \\
\sum_{m=1}^{k-2} b(k, 2, m) \int t^{(m)} t^{k-m-2} y(t) \\
\sum_{m=1}^{k-3} b(k, 3, m) \int^{(m)} t^{k-m-3} y(t) \\
\vdots \\
\sum_{m=1}^{k-d} b(k, d, m) \int_{(m)}^{(m)} t^{k-m-d} y(t)
\end{array}\right)
\end{aligned}
$$

e assim, pode-se escrever (2.16) como:

$$
A(k, t) \bar{y}^{(d)}(t)=x(k, t) .
$$

Ainda, se definirmos:

$$
z_{i}(k, t)=\sum_{m=1}^{k-i} b(k, i, m) \int^{(m)} t^{k-m-i} y(t)
$$

de forma a escrever, para cada elemento de $x(k, t)$ :

$$
x_{i}(k, t)=a(k, d, 0) t^{k-d} y(t)+z_{i}(t, k),
$$

observaremos que as variáveis $z_{i}(t, k)$ obedecem a uma relação diferencial, já que 


$$
\begin{aligned}
\dot{z}_{i}(k, t) & =\sum_{m=1}^{k-i} b(k, i, m) \int^{(m-1)} t^{k-m-i} y(t) \\
& =\sum_{\mu=0}^{k-i-1} b(k, i, \mu+1) \int t^{k-\mu-i-1} y(t) \\
& =b(k, i, 1) t^{k-i-1} y(t)+\sum_{\mu=1}^{k-(i+1)} b(k, i+1, \mu) \int t^{k-\mu-(i+1)} y(t) \\
& =b(k, i, 1) t^{k-i-1} y(t)+z_{i+1}(k, t) .
\end{aligned}
$$

Trata-se de um sistema dinâmico cuja entrada é $y(t)$ e que pode ser entendido uniformemente a uma ordem $k-1$. Os primeiros $i=1, \ldots, d$ estágios, $z_{i}(k, t)$, deste sistema dinâmico são entradas de (2.19), ou seja:

$$
x(k, t)=\left(\begin{array}{c}
a(k, 1,0) t^{k-1} \\
a(k, 2,0) t^{k-2} \\
a(k, 3,0) t^{k-3} \\
\vdots \\
a(k, d, 0) t^{k-d}
\end{array}\right) y(t)+\left(\begin{array}{c}
z_{1}(k, t) \\
z_{2}(k, t) \\
z_{3}(k, t) \\
\vdots \\
z_{d}(k, t)
\end{array}\right)
$$

pela aplicação desta equação, podemos extrair uma fórmula explícita para as derivadas estimadas $\bar{y}^{(d)}(t)$ :

$$
\bar{y}^{(d)}(t)=A^{-1}(k, t) x(k, t)
$$

Como os elementos de $A(k, t)$ são:

$$
[A(k, t)]_{i, j}= \begin{cases}a(k, i, j) t^{k+j-i} & , i \geq j \\ 0 & , i<j\end{cases}
$$

pode-se demonstrar que os elementos correspondentes da matriz inversa, $A^{-1}$ são:

$$
\left[A^{-1}(k, t)\right]_{i, j}= \begin{cases}\left(\begin{array}{c}
k+i-j-1 \\
i-j
\end{array}\right) \frac{(k-j-1) !}{(k-i-1) !} t^{j-i-k} & , i \geq j \\
0 & , i<j\end{cases}
$$


a equação (2.24) se torna:

$$
\begin{aligned}
\tilde{y}^{(i)}(t) & =\sum_{j=1}^{d}\left[A^{-1}(k, t)\right]_{i, j} x_{j}(k, t) \\
& =\sum_{j=1}^{i}\left[A^{-1}(k, t)\right]_{i, j} a(k, j, 0) t^{k-j} \\
& +\sum_{j=1}^{i} \sum_{m=1}^{k-j}\left[A^{-1}(k, t)\right]_{i, j} b(k, j, m) \int^{(m)} t^{k-m-j} y(t) .
\end{aligned}
$$

O primeiro somatório resulta em uma expressão fechada. Para o segundo, o número de integrações pode ser explicitado:

$$
\begin{aligned}
\tilde{y}^{(i)}(t) & =\frac{1}{t^{i}} y(t) \frac{(k-1) !}{(k-i-1) !} \sum_{j=1}^{i}\left(\begin{array}{c}
k \\
j
\end{array}\right)\left(\begin{array}{c}
k+i-j-1 \\
i-j
\end{array}\right)(-1)^{1-j} \\
& +\sum_{m=1}^{k-i} \sum_{j=1}^{i}\left[A^{-1}\right]_{i j} b(k, j, m) \int t^{k-m-j} y(t) \\
& +\sum_{m=k-i+1}^{k-1} \sum_{j=1}^{k-m}\left[A^{-1}\right]_{i j} b(k, j, m) \int^{(m)} t^{k-m-j} y(t)
\end{aligned}
$$

definindo:

$$
c(k, i, j, m) \triangleq\left(\begin{array}{c}
k+i-j-1 \\
i-j
\end{array}\right)\left(\begin{array}{c}
k \\
m+j
\end{array}\right)^{2} \frac{(k-j-1) !}{(k-i-1) !}(-1)^{1-m-j}(m+j) !
$$

resulta uma expressão explícita para a estimação das $i=1, \ldots, d$ derivadas de $y(t)$

$$
\begin{aligned}
\tilde{y}^{(i)}(t) & =\frac{(k+i-1) !}{i !(k-i-1) !} \frac{1}{t^{i}} y(t) \\
& +\sum_{m=1}^{k-i} \sum_{j=1}^{i} c(k, i, j, m) \frac{1}{t^{k+i-j}} \int^{(m)} t^{k-m-j} y(t) \\
& +\sum_{m=k-i+1}^{k-1} \sum_{j=1}^{k-m} c(k, i, j, m) \frac{1}{t^{k+i-j}} \int^{(m)} t^{k-m-j} y(t) .
\end{aligned}
$$

Pela introdução dos estágios do filtro variante no tempo definidos por (2.20) leva ao resul- 
tado principal, uma fórmula explícita para estimar as derivadas de ordens $i=1, \ldots, d$, utilizando filtros variantes no tempo

$$
\tilde{y}^{(i)}(t)=\frac{(k+i-1) !}{i !(k-i-1) !} \frac{1}{t^{i}} y(t)+\sum_{j=1}^{i}\left(\begin{array}{c}
k+i-j-1 \\
i-j
\end{array}\right) \frac{(k-j-1) !}{(k-i-1) !} \frac{1}{t^{k+i-j}} z_{j}(k, t)
$$

A equação (2.31) é o resultado fundamental desenvolvido no artigo [1], de maneira a explicitar a estrutura de um filtro variante no tempo. Para facilitar o estudo destes estimadores quando variamos a ordem do estimador, no presente trabalho usaremos algumas constantes definidas em [1] e definiremos outras, conforme tabela 2.1:

Tabela 2.1: Constantes relativas ao estimador algébrico

\begin{tabular}{|c|c|c|}
\hline Constante & Definição & Propriedades \\
\hline \hline$B(k, i, j)$ & $\left(\begin{array}{c}k \\
i+j\end{array}\right)^{2}(-1)^{1-j-i}(i+j) !$ & Número Inteiro \\
\hline$D(k, i, j)$ & $\left(\begin{array}{c}k+i-j-1 \\
i-j\end{array}\right) \frac{(k-j-1) !}{(k-i-1) !}$ & Número Inteiro positivo ou zero \\
\hline$E(k, i)$ & $\frac{(k+i-1) !}{i !(k-i-1) !}$ & Número Inteiro positivo \\
\hline
\end{tabular}

E assim podemos escrever:

$$
\begin{aligned}
\dot{z}_{i}(k, t) & =B(k, i, 1) t^{k-i-1} y(t)+z_{i+1}(k, t) \\
\tilde{y}^{(i)}(t) & =E(k, i) \frac{1}{t^{i}} y(t)+\sum_{j=1}^{i} D(k, i, j) \frac{1}{t^{k+i-j}} z_{j}(k, t)
\end{aligned}
$$

ou, como $D(k, i, j)=0 \forall j>i$, podemos escrever em notação matricial: 


$$
\begin{aligned}
& \left(\begin{array}{c}
\dot{z}_{1}(k, t) \\
\dot{z}_{2}(k, t) \\
\vdots \\
\dot{z}_{k-2}(k, t) \\
\dot{z}_{k-1}(k, t)
\end{array}\right)=\left(\begin{array}{c}
B(k, 1,1) t^{k-2} \\
B(k, 2,1) t^{k-3} \\
\vdots \\
B(k, k-2,1) t^{1} \\
B(k, k-1,1) t^{0}
\end{array}\right) y(t)+\left(\begin{array}{c}
z_{2}(k, t) \\
z_{3}(k, t) \\
\vdots \\
z_{k-1}(k, t) \\
0
\end{array}\right) \\
& \left(\begin{array}{c}
\tilde{y}^{(1)}(t) \\
\tilde{y}^{(2)}(t) \\
\vdots \\
\tilde{y}^{(k-2)}(t) \\
\tilde{y}^{(k-1)}(t)
\end{array}\right)=\left(\begin{array}{c}
\frac{E(k, 1)}{t} \\
\frac{E(k, 2)}{t^{2}} \\
\vdots \\
\frac{E(k, k-2)}{E\left(t^{k-2}\right.} \\
\frac{E(k, 1)}{t^{k-1}}
\end{array}\right) y(t)+ \\
& +\left(\begin{array}{ccccc}
\frac{D(k, 1,1)}{t^{k}} & \frac{D(k, 1,2)}{t^{k-1}} & \cdots & \frac{D(k, 1, k-2)}{t^{3}} & \frac{D(k, 1, k-1)}{t^{2}} \\
\frac{D(k, 2,1)}{t^{k+1}} & \frac{D(k, 2,2)}{t^{k}} & \cdots & \frac{D(k, 2, k-2)}{t^{4}} & \frac{D(k, 2, k-1)}{t^{3}} \\
\vdots & \vdots & \ddots & \vdots & \vdots \\
\frac{D(k, k-2,1)}{t^{2 k-3}} & \frac{D(k, k-2,2)}{t^{2 k-4}} & \cdots & \frac{D(k, k-2, k-2)}{D(k, k-2,1)} & \frac{D(k, k-2, k-1)}{t^{k}} \\
\frac{t^{2 k-2}}{t^{2 k-2}} & \frac{\cdots}{t^{2 k-3}} & \frac{D(k, k-2, k-2)}{t^{k+1}} & \frac{D(k, k-2, k-1)}{t^{k}}
\end{array}\right)\left(\begin{array}{c}
z_{1}(k, t) \\
z_{2}(k, t) \\
\vdots \\
z_{k-2}(k, t) \\
z_{k-1}(k, t)
\end{array}\right) \text { (2.35) }
\end{aligned}
$$

\subsubsection{Exemplo algébrico de ordem baixa}

Para ilustrar a aplicabilidade desta teoria, vamos desenvolver as equações do estimador para uma série de Taylor de ordem 3, possibilitando estimar a primeira e a segunda derivadas de um sinal definido por um polinômio de grau 3

As constantes serão:

\begin{tabular}{|c|c|}
\hline Constante & Valor \\
\hline \hline$B_{1}$ & -18 \\
\hline$B_{2}$ & 6 \\
\hline$D_{1,1}$ & 1 \\
\hline$D_{1,2}$ & 0 \\
\hline$D_{2,1}$ & 3 \\
\hline$D_{2,2}$ & 1 \\
\hline$E_{1}$ & 6 \\
\hline$E_{2}$ & 12 \\
\hline
\end{tabular}

Tabela 2.2: Constantes para estimador de ordem 3.

O filtro variante no tempo fica então definido por: 


$$
\begin{array}{lcc}
\dot{Z}_{1}(t) & = & -18 y(t) t^{1}+Z_{2}(t) \\
\dot{Z}_{2}(t) & = & 6 y(t)
\end{array}
$$

E as derivadas serão estimadas por:

$$
\begin{array}{llc}
y^{(1)}(t) & = & \frac{6}{t} y(t)+\frac{1}{t^{3}} Z_{1}(t) \\
y^{(2)}(t) & =\frac{12}{t^{2}} y(t)+\frac{3}{t^{4}} Z_{1}(t)+\frac{1}{t^{3}} Z_{2}(t)
\end{array}
$$

Para testar a funcionalidade deste filtro, vamos supor um sinal $y(t)=\alpha_{2} t^{2}+\alpha_{1} t+\alpha_{0}$ para o qual desejamos estimar a primeira e segunda derivadas, $y^{(1)}(t)=2 \alpha_{2} t+\alpha_{1}$ e $y^{(2)}(t)=2 \alpha_{2}$.

Resolvendo a equação do estágio $Z_{2}$ do filtro variante no tempo, $\operatorname{com} Z_{2}(0)=0$ :

$\dot{Z}_{2}(t)=6 y(t)$

$\dot{Z}_{2}(t)=6\left(\alpha_{2} t^{2}+\alpha_{1} t+\alpha_{0}\right)$

$\dot{Z}_{2}(t)=6 \alpha_{2} t^{2}+6 \alpha_{1} t+6 \alpha_{0}$

$Z_{2}(t)=2 \alpha_{2} t^{3}+3 \alpha_{1} t^{2}+6 \alpha_{0} t$

Resolvendo a equação do estágio $Z_{1}$ do filtro variante no tempo, com $Z_{1}(0)=0$ :

$\dot{Z}_{1}(t)=-18 y(t) t^{1}+Z_{2}(t)$

$\dot{Z}_{1}(t)=-18\left(\alpha_{2} t^{2}+\alpha_{1} t+\alpha_{0}\right) t^{1}+2 \alpha_{2} t^{3}+3 \alpha_{1} t^{2}+6 \alpha_{0} t$

$\dot{Z}_{1}(t)=\left(-18 \alpha_{2} t^{3}-18 \alpha_{1} t^{2}-18 \alpha_{0} t\right)+2 \alpha_{2} t^{3}+3 \alpha_{1} t^{2}+6 \alpha_{0} t$

$\dot{Z}_{1}(t)=-16 \alpha_{2} t^{3}-15 \alpha_{1} t^{2}-12 \alpha_{0} t$

$Z_{1}(t)=-4 \alpha_{2} t^{4}-5 \alpha_{1} t^{3}-6 \alpha_{0} t^{2}$

A estimativa da primeira derivada nos leva a:

$y^{(1)}(t)=\frac{6}{t} y(t)+\frac{1}{t^{3}} Z_{1}(t)$

$y^{(1)}(t)=\frac{6}{t}\left(\alpha_{2} t^{2}+\alpha_{1} t+\alpha_{0}\right)+\frac{1}{t^{3}}\left(-4 \alpha_{2} t^{4}-5 \alpha_{1} t^{3}-6 \alpha_{0} t^{2}\right)$

$y^{(1)}(t)=6 \alpha_{2} t^{1}+6 \alpha_{1}+6 \alpha_{0} \frac{1}{t}-4 \alpha_{2} t^{1}-5 \alpha_{1}-6 \alpha_{0} \frac{1}{t}$

$y^{(1)}(t)=2 \alpha_{2} t+\alpha_{1}$

E da segunda derivada:

$y^{(2)}(t)=\frac{12}{t^{2}} y(t)+\frac{3}{t^{4}} Z_{1}(t)+\frac{1}{t^{3}} Z_{2}(t)$ 


$$
\begin{aligned}
& y^{(2)}(t)=\frac{12}{t^{2}}\left(\alpha_{2} t^{2}+\alpha_{1} t+\alpha_{0}\right)+\frac{3}{t^{4}}\left(-4 \alpha_{2} t^{4}-5 \alpha_{1} t^{3}-6 \alpha_{0} t^{2}\right)+\frac{1}{t^{3}}\left(+2 \alpha_{2} t^{3}+3 \alpha_{1} t^{2}+6 \alpha_{0} t\right) \\
& y^{(2)}(t)=12 \alpha_{2}+12 \alpha_{1} \frac{1}{t}+12 \alpha_{0} \frac{1}{t^{2}}-12 \alpha_{2}-15 \alpha_{1} \frac{1}{t}-18 \alpha_{0} \frac{1}{t^{2}}+2 \alpha_{2}+3 \alpha_{1} \frac{1}{t}+6 \alpha_{0} \frac{1}{t^{2}} \\
& y^{(2)}(t)=12 \alpha_{2}-12 \alpha_{2}+2 \alpha_{2}+12 \alpha_{1} \frac{1}{t}-15 \alpha_{1} \frac{1}{t}+3 \alpha_{1} \frac{1}{t}+12 \alpha_{0} \frac{1}{t^{2}}-18 \alpha_{0} \frac{1}{t^{2}}+6 \alpha_{0} \frac{1}{t^{2}} \\
& y^{(2)}(t)=2 \alpha_{2}
\end{aligned}
$$

Como se pode observar, as estimativas são exatamente as derivadas reais, obtidas a partir da soma ponderada do sinal e de suas integrais no tempo.

\subsection{Particularidades da teoria de estimadores rápidos}

É claro que a técnica descrita possui limitações. Ao se analisar a equação das derivadas (2.31) observa-se facilmente que não existe solução para $t=0$. Entretanto após este instante de singularidade, a estimativa teórica existe e é valida. Na prática entretanto, ao se realizar estas operações com auxílio de um computador, ou seja, com uma precisão numérica finita, observaremos que o resultado desta equação não será válido dentro de um pequeno intervalo onde $t \in[0, \varepsilon)$.

O estimador é construído supondo um sinal polinômial de grau $k$, o que não acontece na prática. Embora o sinal de entrada não seja perfeitamente polinomial, ainda assim pode ser bem descrito por um polinômio de grau $k$ por um certo intervalo de tempo. Tem-se então que as derivadas estimadas têm também um intervalo de validade e, quando nos aproximamos do limiar desta janela de tempo o estimador deverá ser reinicializado. Em [8] discute-se detalhadamente o erro de estimação para um sinal senoidal, mas no presente trabalho a analise deste erro não sera considerada.

Este procedimento de reinicialização do estimador consiste apenas em zerar todos os estágios do filtro $Z$ e fazer com que o tempo dentro do algoritmo de estimação seja também transladado a zero.

O critério para reinicializar o estimador pode ser baseado em algum critério de erro acumulado, ou simplesmente escolher um intervalo fixo. Neste trabalho utilizaremos um intervalo fixo para realizar o "reset" do estimador. Não há procedimento bem definido para nortear a escolha deste intervalo, mas ele afeta, como veremos adiante, a banda passante do estimador e, desta maneira, se precisamos detectar as derivadas de sinais com freqüências mais elevadas, devemos reduzir o intervalo de reset. 


\subsection{Uso de dois estimadores em paralelo}

Conforme observaremos adiante a banda passante do estimador vai se reduzindo com o passar do tempo, conseqüentemente aumenta-se sua capacidade de rejeitar ruídos de alta freqüência. Veremos que existe uma região de bom compromisso entre a validade da estimativa e a rejeição de ruídos de alta freqüência. Para se explorar ao máximo esta região vamos operar, seguindo [2], um esquema com dois estimadores em paralelo e uso de "reset" defasado, de forma e sempre ter um estimador em seu melhor intervalo de estimação. A figura 2.1 demonstra a idéia básica:

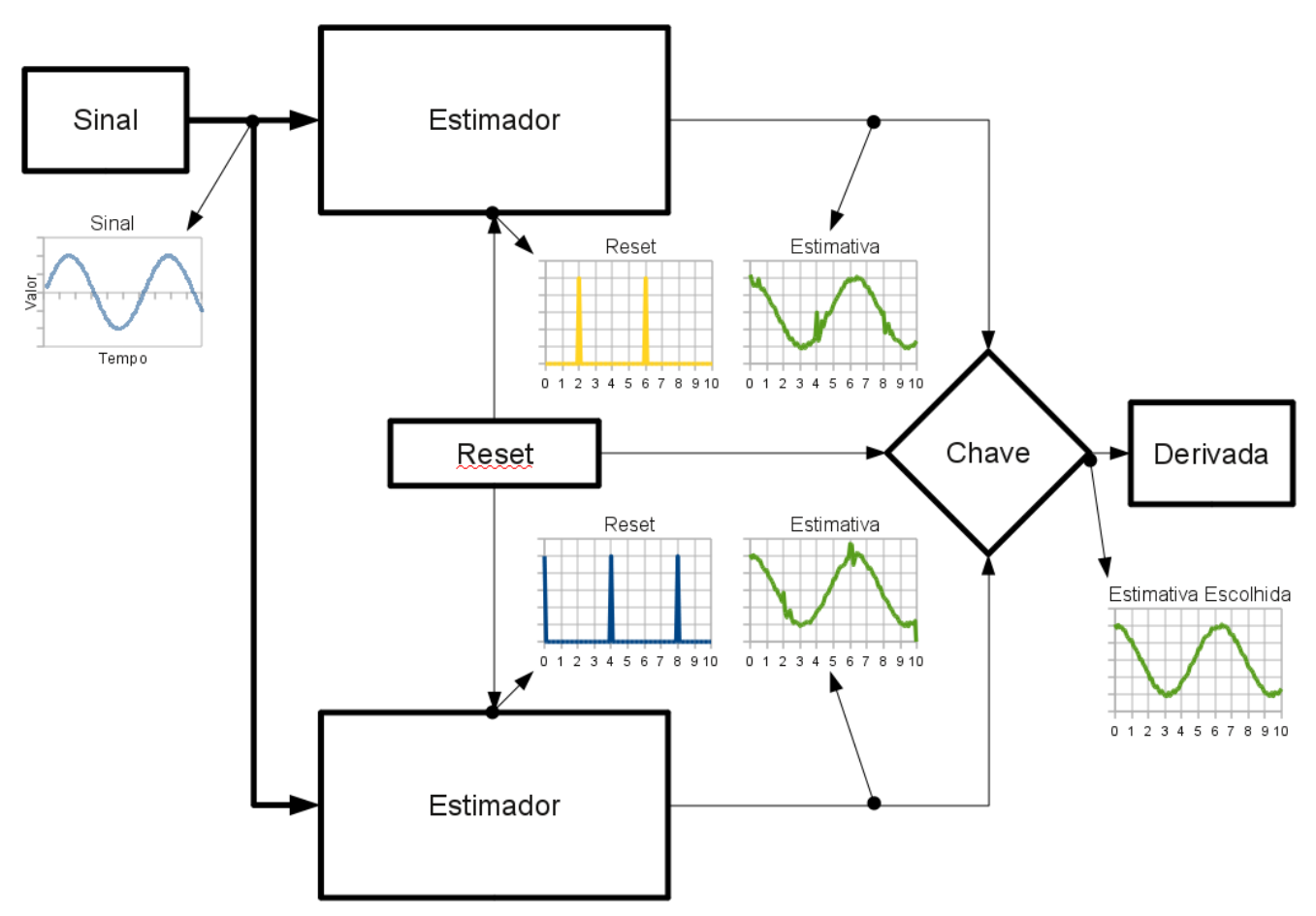

Figura 2.1: Esquema de chaveamento entre dois estimadores.

\subsection{Uso de filtragem adicional}

Em [2] discute-se ainda a utilização conjunta de uma filtragem adicional, invariante no tempo. O uso de filtragem adicional pode reduzir os ruídos mas ocasiona um atraso de fase.

$\mathrm{Na}$ implementação em tempo discreto, utilizamos uma aproximação polinomial que é de uma certa maneira, uma filtragem adicional invariante no tempo e que só foi empregada para permitir a implementação em um sistema de controle de tempo real. No presente trabalho não faremos uso de qualquer outro tipo adicional de filtragem, de modo a exibir o mais fielmente 
possível o comportamento fundamental dos estimadores rápidos.

\subsection{Simulações}

Seguem algumas simulações de diversos sinais, íntegros e corrompidos com ruído. O objetivo destas simulações é identificar características e o comportamento dos estimadores algébricos de diferentes ordens na estimação de derivadas de sinais diversos.

Nos ensaios a seguir, utilizou-se um estimador de ordem $k=7$.

O primeiro ensaio, figura 2.3, se refere a uma função seno, $y(t)=\sin (t)$. A derivada é estimada corretamente quase que imediatamente após o instante $t=0$ e um valor próximo em avaliação meramente visual é obtido até aproximadamente oito segundos.

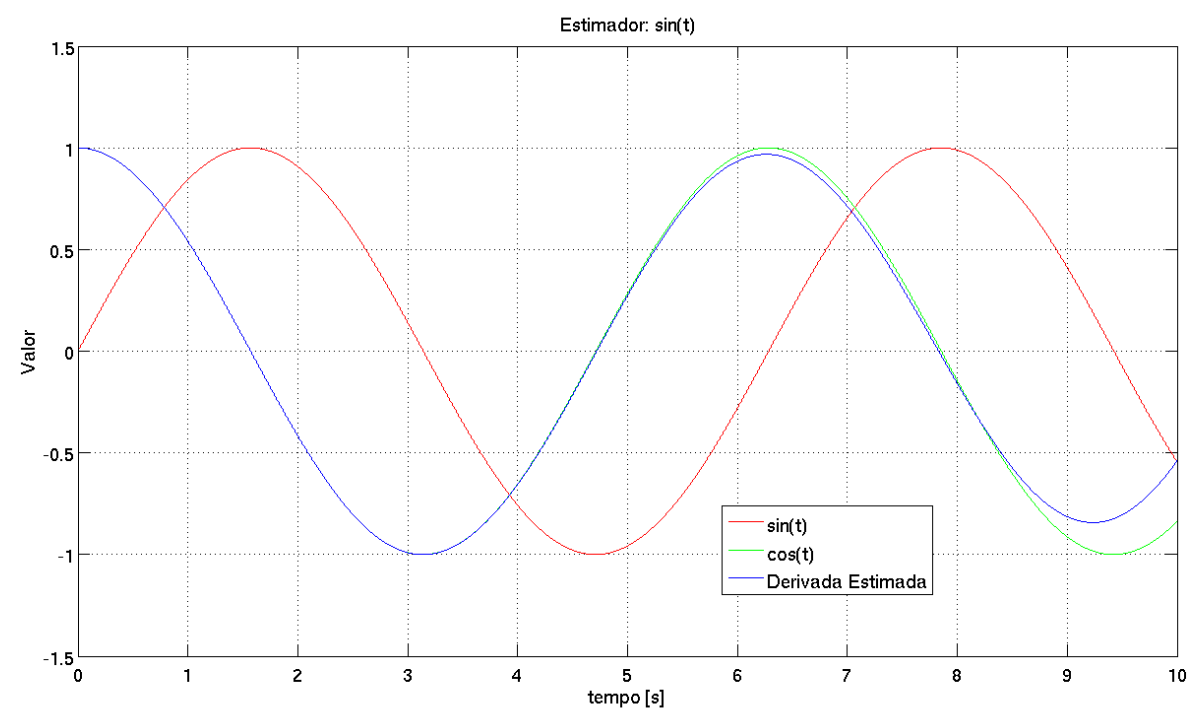

Figura 2.2: Estimando a primeira derivada de $y(t)=\sin (t)$.

Para uma função $y(t)=\sin (2 t)$, a validade da estimativa se verifica até aproximadamente quatro segundos, como pode ser visualizado na figura 2.3. 


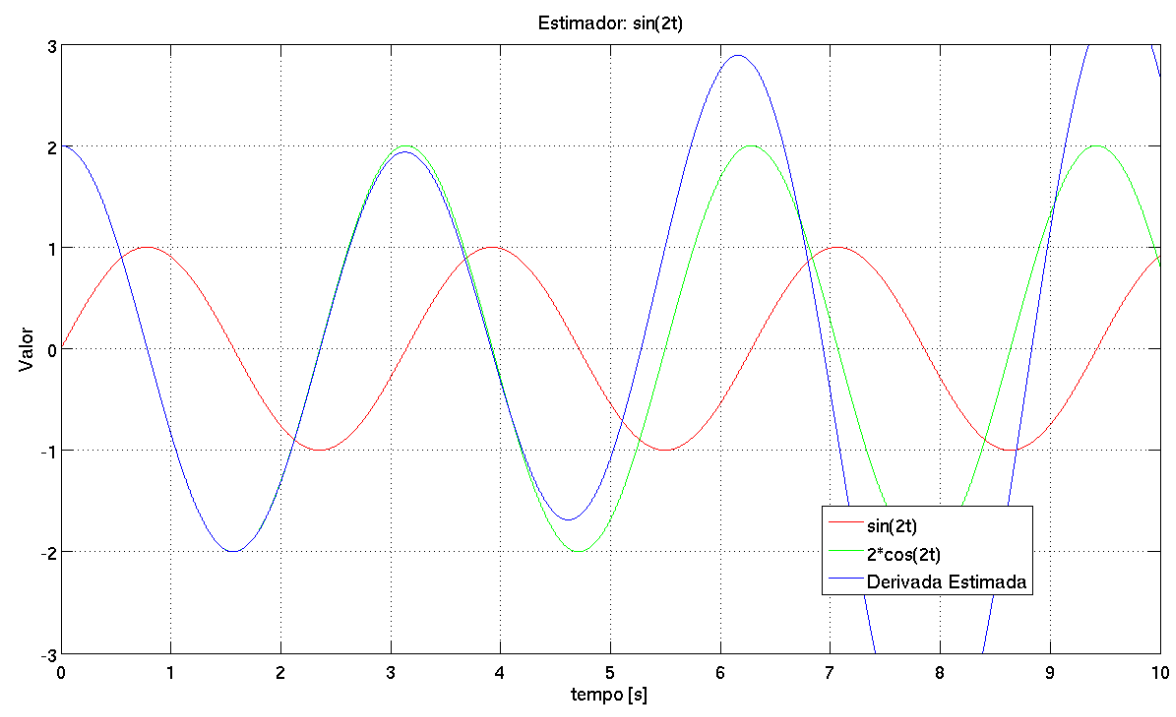

Figura 2.3: Estimando a primeira derivada de $y(t)=\sin (t)$.

Na simulação a seguir, figuras 2.42 .5 e 2.6, o sinal de entrada foi uma composição de senos, $y(t)=\sin (t)+\frac{\sin (100 t)}{1000}+\frac{\sin (10000 t)}{1000000}$. A primeira derivada desta função é dada por $y^{(1)}(t)=\cos (t)+\frac{\cos (100 t)}{10}+\frac{\cos (10000 t)}{100}$, mas para alguns instantes logo após $t=0$, pode ser aproximada por $1+\frac{\cos (100 t)}{10}+\frac{\cos (10000 t)}{100}$ ou $1.1+\frac{\cos (10000 t)}{100}$.

Observa-se, pelas figuras, que a estimativa é aceitável por praticamente oito segundos para a derivada do termo $\sin (t)$ e que as derivadas das parcelas $\frac{\sin (100 t)}{1000} \mathrm{e} \frac{\sin (10000 t)}{1000000}$ foram bem estimadas até $8 \cdot 10^{-2}$ e $8 \cdot 10^{-4}$ segundos respectivamente. Estas informações nos demonstram que o tempo de reinicialização está intimamente associado à largura de banda desejada, e que com o passar do tempo, a banda passante do estimador diminui. 


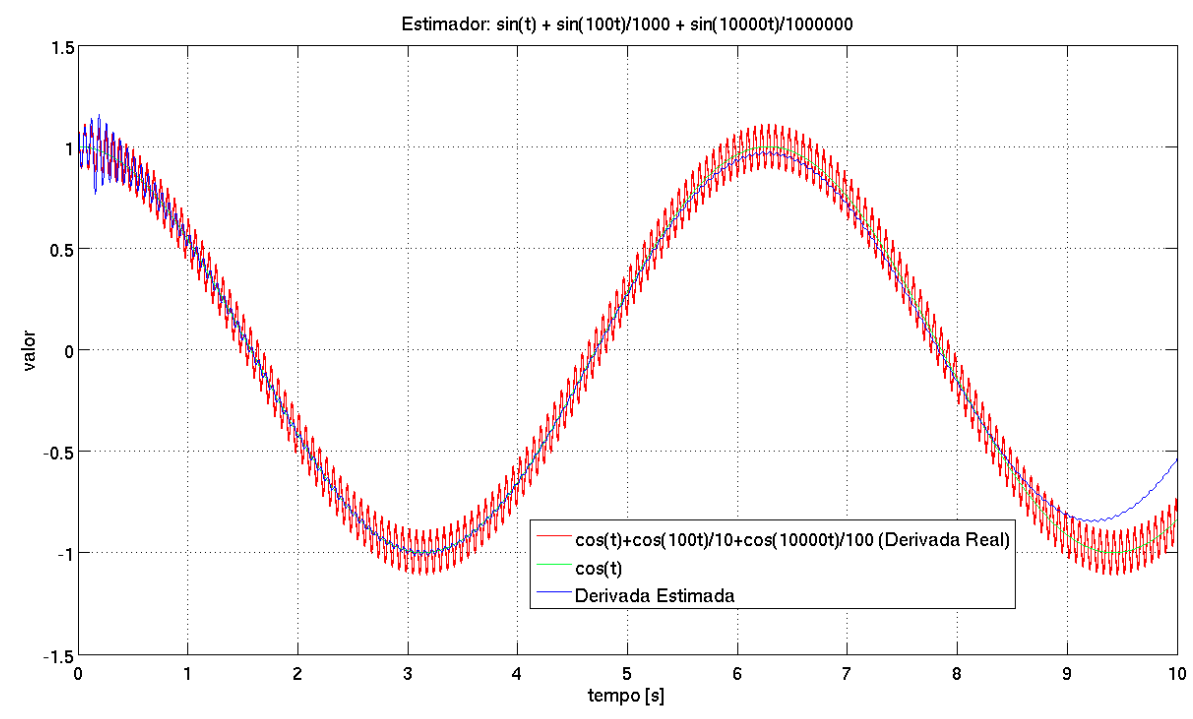

Figura 2.4: Estimando a primeira derivada de $y(t)=\sin (t)+\frac{\sin (100 t)}{1000}+\frac{\sin (10000 t)}{1000000}$.

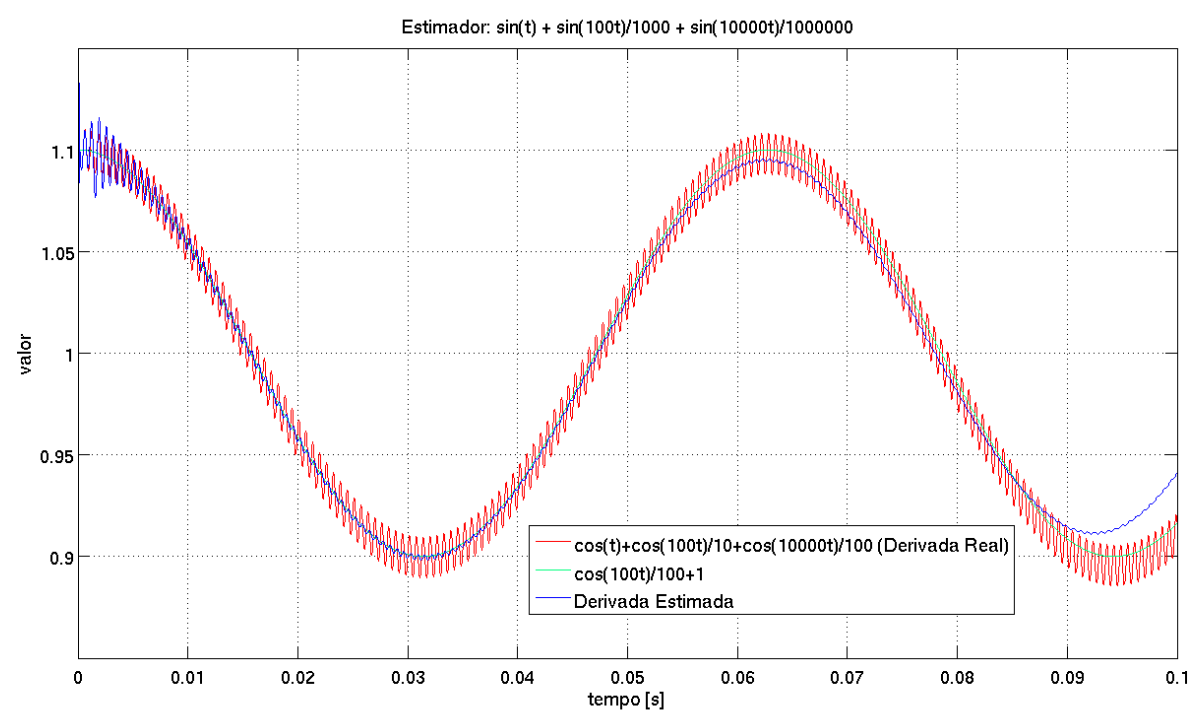

Figura 2.5: Detalhe da estimativa da primeira derivada de $y(t)=\sin (t)+\frac{\sin (100 t)}{1000}+$ $\frac{\sin (10000 t)}{1000000}$. 


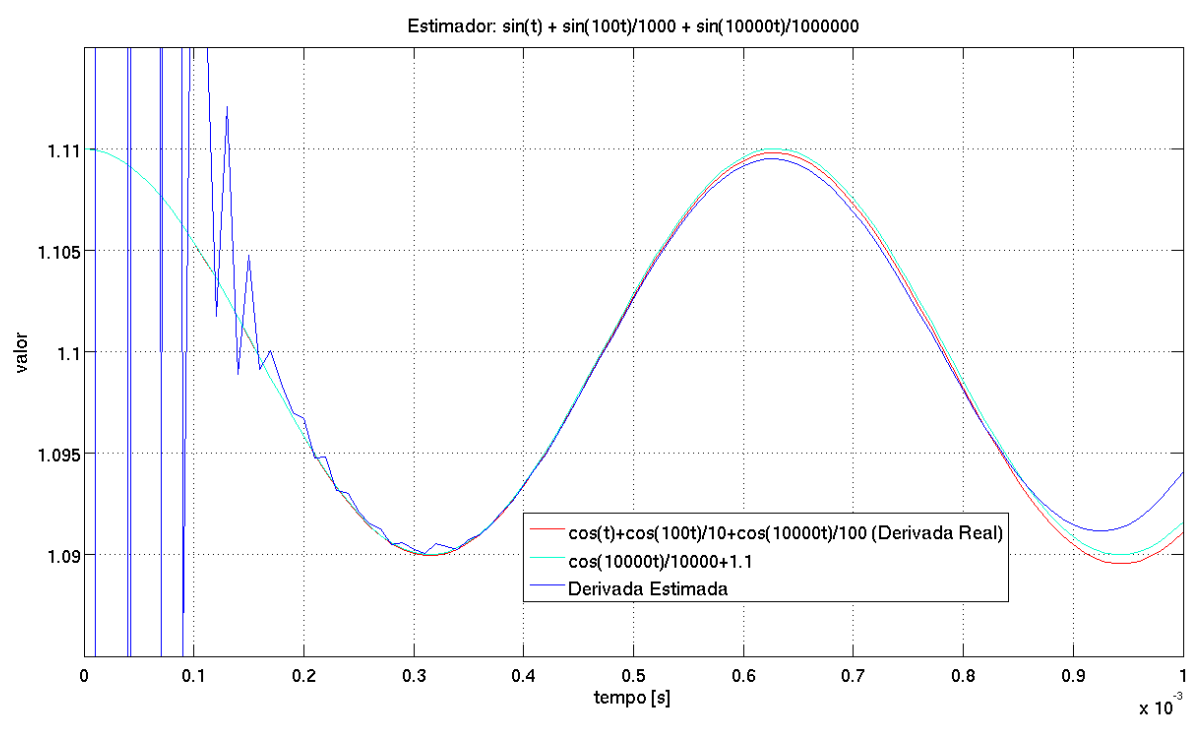

Figura 2.6: Detalhe da estimativa da primeira derivada de $y(t)=\sin (t)+\frac{\sin (100 t)}{1000}+$ $\frac{\sin (10000 t)}{1000000}$.

O comportamento do estimador proposto foi analisado também quando o sinal está comprometido por ruído. Para as simulações a seguir, com o intuito de obter-se uma função geradora de ruído contínuo, não nos utilizamos das funções usuais do software Matlab. O ruído foi gerado por uma soma de dez mil senóides com freqüências escolhidas aleatoriamente dentro de um determinado intervalo, assim obteve-se uma função contínua que aproxima o ruído branco e que pode ser integrada numericamente pela rotina ode45. A figura 2.7 ilustra o resultado obtido.

Novamente, observa-se o estreitamento da banda passante do estimador com o passar do tempo, resultando em uma atenuação progressiva do ruído. 


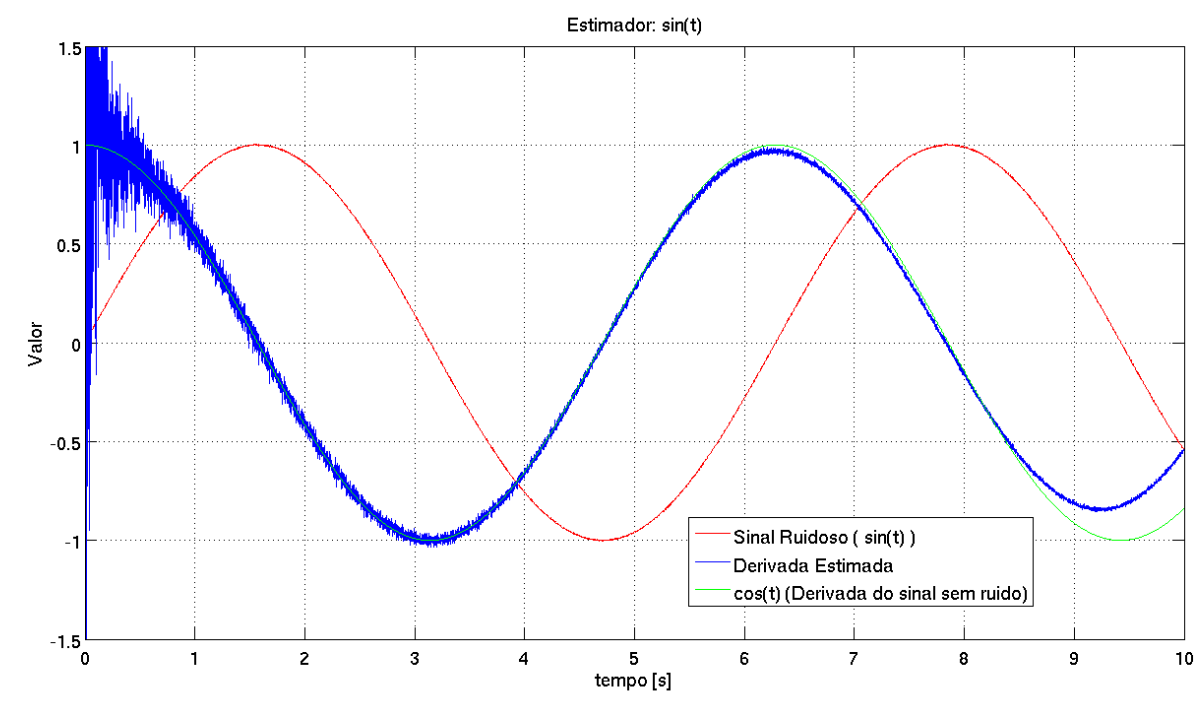

Figura 2.7: Estimando a primeira derivada de $y(t)=\sin (t)$ quando comprometido por ruído.

Fica evidente que existe um compromisso entre a atenuação de ruídos de alta freqüência e a precisão das derivadas estimadas, o intervalo de reset deve ser escolhido de forma chegar a uma boa atenuação de ruídos mantendo as estimativas válidas. Aumentando o tempo de reset chegamos a uma menor banda passante à medida que o tempo passa e isto leva a uma maior atenuação do ruído, porém diminuímos a precisão de estimação. 


\title{
3 Implementação em tempo discreto
}

\author{
“"Eu... eu sou uma menininha', respondeu Alice, bastante insegura, \\ lembrando-se do número de mudanças que sofrera aquele dia. \\ 'Realmente uma história muito plausível!' disse a Pomba num tom \\ do mais profundo desprezo, 'Vi muitas menininhas no meu tempo, \\ mas nunca uma com um pescoço desse!
} Não, não! Você é uma cobra; e não adianta negar. Suponho agora que vai dizer que nunca provou um ovo!' 'Provei ovos, sem dúvida', disse Alice, que era uma criança muito sincera; 'mas meninas comem quase tantos ovos quanto as cobras, sabe'.

'Não acredito nisso', declarou a Pomba; 'mas, se comem, então são uma espécie de cobra, é só o que posso dizer'.”

(CARROL, 2002, p. 51-53)

Aqui descreve-se os problemas enfrentados para implementar a metodologia proposta no capítulo anterior, uma análise do efeito da integração numérica sobre o filtro variante no tempo. Em seguida propomos uma implementação baseada em aproximação polinomial, capaz de superar estas dificuldades, e desenvolvimento de fórmulas para pré-calcular parâmetros que facilitarão a implementação em tempo real.

Apresentamos ainda um pequeno exemplo de controle que comprova a aplicabilidade desta metodologia. 


\subsection{Dificuldades relativas à implementação}

Implementar a teoria descrita no capitulo anterior e, desta maneira, utilizá-la em sistemas de controle em tempo real não é uma tarefa trivial. Operações de multiplicação e divisão por potências do tempo decorrido são necessárias e muito difíceis de construir apenas com circuitos analógicos. A solução imediata seria então utilizar um computador para efetuar estes cálculos. A implementação com auxílio de um computador envolve logicamente a discretização do processo, além de obter-se a resposta do filtro variante no tempo através de algum método como a integração trapezoidal de Euler ou métodos de Runge-Kutta.

Em ensaios preliminares, entretanto, observou-se que para alcançar uma rápida convergência destas equações diferenciais, o passo de integração deve ser extremamente reduzido nas proximidades de $t=0$, implicando em uma taxa de amostragem exageradamente elevada. Para ilustrar tal observação, a figura 3.1 exibe os resultados da primeira derivada de um sinal senoidal. Utilizou-se diversos intervalos de integração numérica no método de Runge-Kutta de quarta ordem e diferentes resultados foram obtidos para cada passo de integração. Na figura 3.2 demonstra-se mais detalhadamente a convergência numérica. Na figura 3.3 apresentamos os erros absolutos.

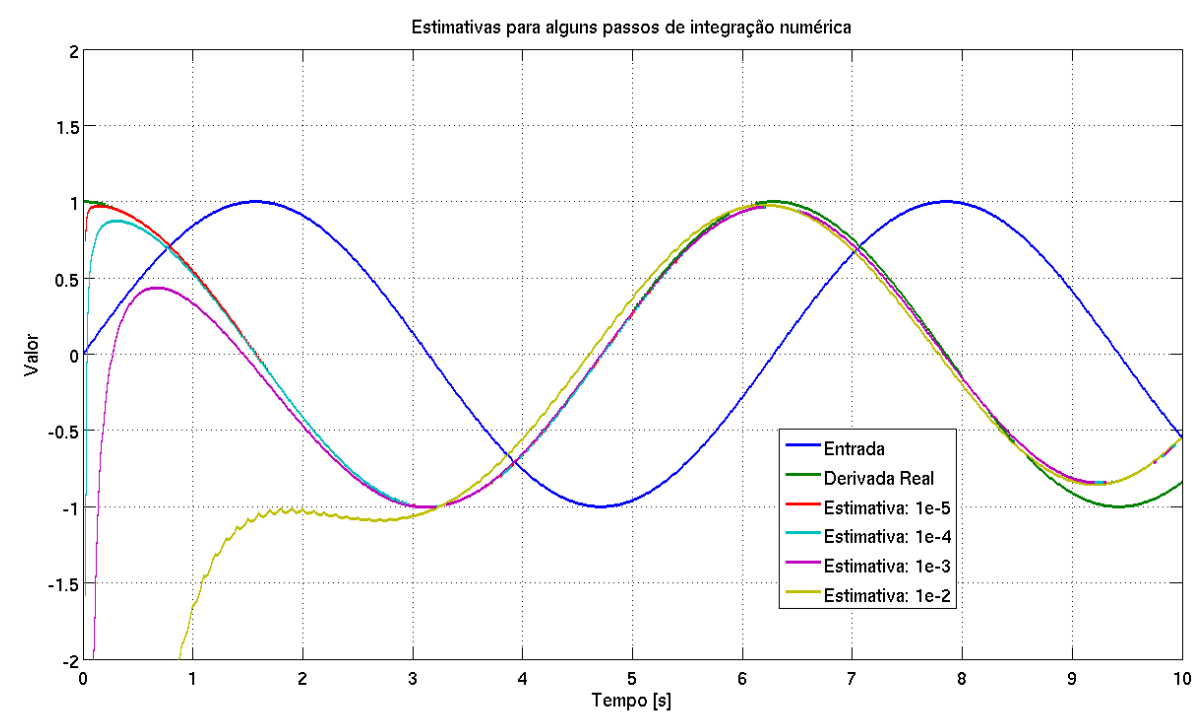

Figura 3.1: Resultado da integração numérica para diferentes passos de integração (de 1e-2 a $1 \mathrm{e}-5)$. 


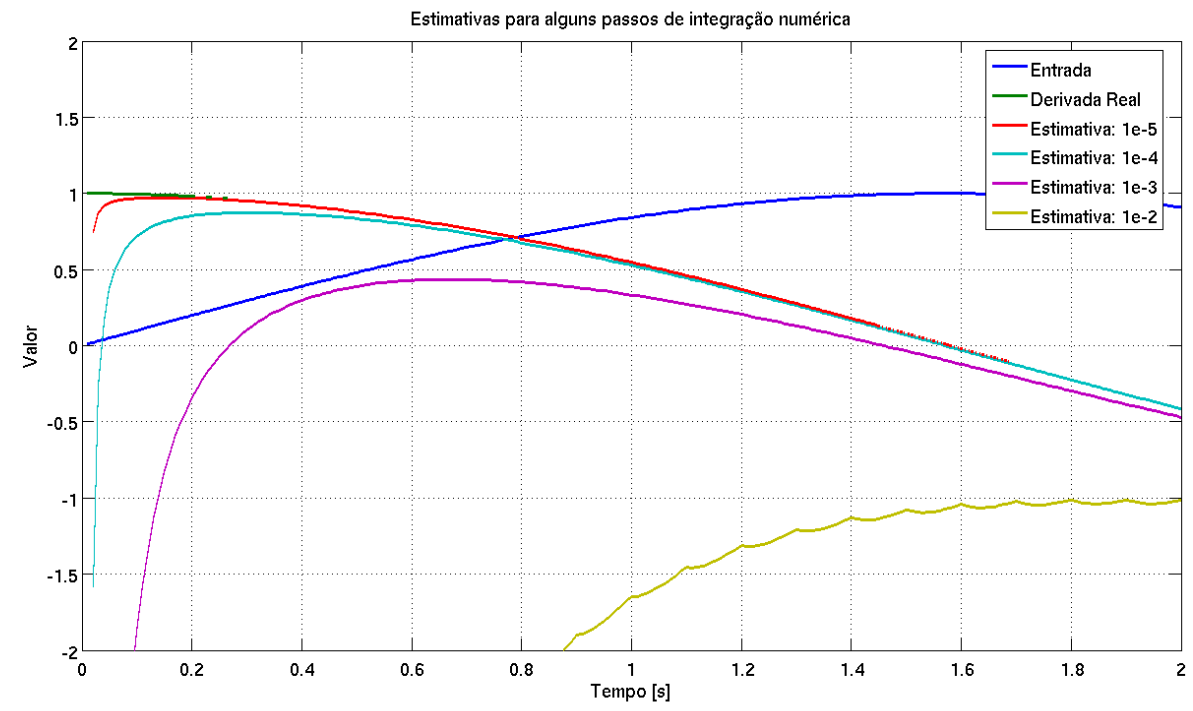

Figura 3.2: Detalhamento da convergência da integração numérica

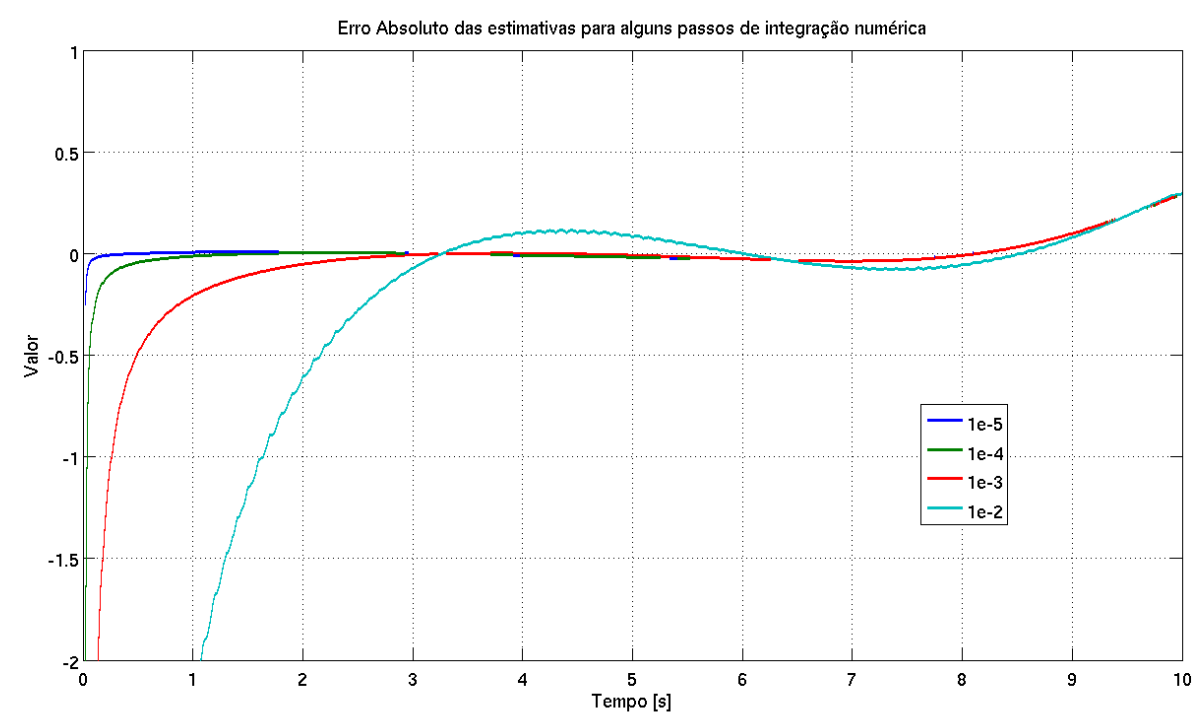

Figura 3.3: Erro absoluto da integração numérica em função do passo de integração.

Nesta simulação comprova-se que para obter um resultado rápido, foi preciso uma taxa de amostragem muito elevada, da ordem de 10000 amostras por segundo para estimar a derivada de um sinal senoidal com freqüência de apenas $\frac{1}{2 \pi}$.

Por certo os resultados relativos ao tempo de convergência não são encorajadores, e em [3], aborda-se uma solução viabilizadora, que constitui contribuição teórica deste trabalho. 


\subsection{Abordagem via aproximação do sinal amostrado por seg- mentos de polinômios}

O resultado fundamental de [3] viabiliza a construção da teoria de estimadores algébricos em sistemas de controle de tempo real, sem entretanto fazer uso de elevada taxa de amostragem. Basicamente, os últimos $p+1$ pontos amostrados do sinal cujas derivadas serão estimadas são aproximados por um polinômio de grau $p$. Posteriormente, a equação do filtro é resolvida, num dado intervalo de amostragem, usando os coeficientes do polinômio que aproxima o sinal de entrada, obtendo-se um resultado analítico exato.

Fazendo, para cada intervalo de amostragem, a entrada $y(t)$ ser aproximada por um polinômio de ordem $p$ dado por:

$$
y(t) \approx \sum_{g=0}^{p} \alpha_{g} t^{g}
$$

E utilizando o resultado, demonstrado no anexo A.1:

Seja uma função $f\left(t_{0}+\Delta_{t}\right)$ definida por uma integral múltipla:

$$
f\left(t_{0}+\Delta_{t}\right) \triangleq \int_{t_{0}}^{t_{0}+\Delta_{t}} \cdots \int \tau^{m} d \tau^{n}
$$

onde $m \in \mathbb{N}^{+}, n \in \mathbb{N}^{+}$e constantes de integração nulas, então:

$$
f\left(t_{0}+\Delta_{t}\right)=\sum_{j=0}^{m} \frac{m !}{j !(m+n-j) !} t_{0}^{j} \Delta_{t}^{m+n-j}
$$

conforme demonstrado em [3] e no anexo A.2, a equação do filtro fica reescrita como:

$$
\begin{aligned}
Z_{l}\left(t_{0}+\Delta_{t}\right) & =\sum_{g=0 h=1}^{p} \sum_{j}^{k-l}\left[\alpha_{g} \sum_{j=0}^{k+g-h-l} B_{l-1+h} \times\right. \\
& \times \frac{(k+g-h-l) !}{j !(k+g-l-j) !} \Delta_{t}^{k+g-l-j} t_{0}^{j}+ \\
& \left.+\quad Z_{l+h}\left(t_{0}\right) \frac{\Delta_{t}^{h}}{h !}\right]+Z_{l}\left(t_{0}\right)
\end{aligned}
$$

onde $\Delta_{t}$ é o intervalo entre as amostras, $\alpha_{g}$ são os coeficientes do polinômio pelo qual se aproxima o sinal de entrada, $t_{o}$ é o instante da amostra que é processada e $B_{l}$ são as constantes já definidas na equação do filtro variante no tempo. 
Desta maneira, é possível utilizar a teoria de estimadores não assintóticos em sistemas de controle de tempo real. $\mathrm{O}$ algoritmo se resume a calcular o polinômio que aproxima a entrada a partir da seguinte matriz:

$$
\left(\begin{array}{c}
y\left(t_{0}\right) \\
y\left(t_{1}\right) \\
y\left(t_{2}\right) \\
\vdots \\
y\left(t_{p}\right)
\end{array}\right)=\left(\begin{array}{ccccc}
t_{0}^{0} & t_{0}^{1} & t_{0}^{2} & \cdots & t_{0}^{p} \\
t_{1}^{0} & t_{1}^{1} & t_{1}^{2} & \cdots & t_{1}^{p} \\
t_{2}^{0} & t_{2}^{1} & t_{2}^{2} & \cdots & t_{2}^{p} \\
\vdots & \vdots & \vdots & \vdots & \vdots \\
t_{p}^{0} & t_{p}^{1} & t_{p}^{2} & \cdots & t_{p}^{p}
\end{array}\right)\left(\begin{array}{c}
\alpha_{0} \\
\alpha_{1} \\
\alpha_{2} \\
\vdots \\
\alpha_{p}
\end{array}\right)
$$

Onde $t_{1}, t_{2}, \ldots, t_{p}=t_{n}$ deve ser lido como $t_{0}-n \Delta_{t}$ e $\alpha_{n}$ são os coeficientes do polinômio de grau $p$

A matriz é quadrada e sempre invertível devido a sua construção como uma matriz de Vandermonde. Então, para cada $t_{0}$, os coeficientes do polinômio serão uma particular combinação linear da amostra atual e das anteriores.

$$
\left(\begin{array}{c}
\alpha_{0} \\
\alpha_{1} \\
\alpha_{2} \\
\vdots \\
\alpha_{p}
\end{array}\right)=\mathbb{A}\left(t_{0}\right)\left(\begin{array}{c}
y\left(t_{0}\right) \\
y\left(t_{1}\right) \\
y\left(t_{2}\right) \\
\vdots \\
y\left(t_{p}\right)
\end{array}\right)
$$

Cada $\mathbb{A}\left(t_{0}\right)$ pode ser previamente calculada, o que será útil para reduzir o tempo de processamento para cada amostra.

Como o intervalo de amostragem é fixo, as potências de $\Delta_{t}$ também podem ser calculadas previamente. A cada amostra, $t_{0}$ será diferente, mas sempre um múltiplo inteiro de $\Delta_{t}$, de forma que ele próprio e suas potências podem também ser pré calculadas. $\mathrm{O}$ algoritmo deve fazer uso da transformação linear adequada $\mathbb{A}$ para obter o polinômio que aproxima a entrada, tomar o valor de $Z\left(t_{0}\right)$ e fazer os cálculos da equação (3.2).

\subsubsection{Comparando a técnica descrita com a integração numérica de um sistema caótico}

As duas abordagens foram usadas para estimar o estado $x_{1}$ do sistema caótico que segue (trata-se do mesmo sistema descrito em [2]: 


$$
\begin{array}{rlc}
\dot{x}_{1} & = & -x_{2}-x_{3} \\
\dot{x}_{2} & = & x_{1}+\frac{x_{2}}{5} \\
\dot{x}_{3} & = & \frac{1}{5}+x_{1} x_{3}-5 x_{3}
\end{array}
$$

usando $x_{2}$ e suas derivadas, podemos estimar $x_{1}$ e $x_{3}$ :

$$
\begin{array}{llc}
x_{1} & = & \dot{x}_{2}-\frac{x_{2}}{5} \\
x_{2} & = & x_{2} \\
x_{3} & = & -x_{2}+\frac{\dot{x}_{2}}{5}-\ddot{x}_{2}
\end{array}
$$

Ambos os estimadores (discreto e contínuo) foram simulados na ausência de pertubações e na presença de ruído aditivo com variância de $1 e-5$, uma taxa de amostragem de $1 e-2$ segundos e intervalo de reset de 0.5 segundos. Adicionalmente, aplicamos uma quantização de $\frac{1}{1024}$ no estimador discreto. As figuras 3.4, 3.5, 3.6, 3.7, 3.8, 3.9, 3.10 e 3.11 ilustram os resultados.

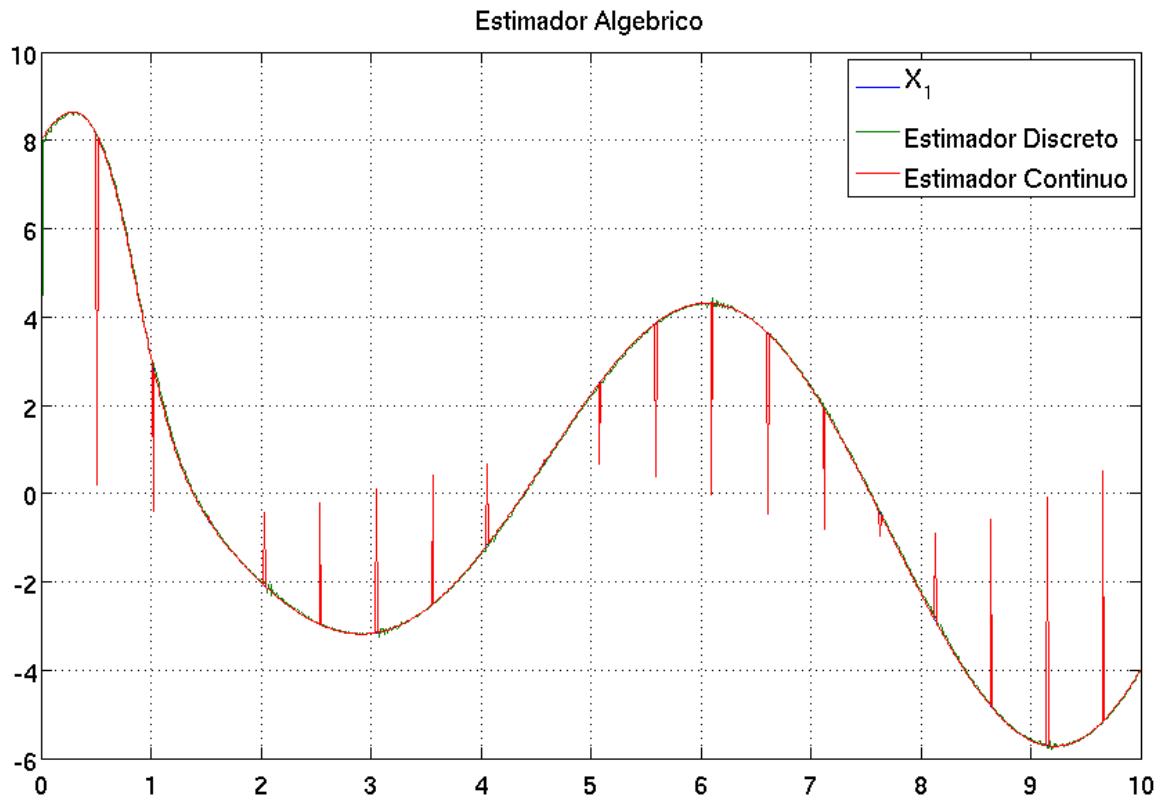

Figura 3.4: Estimador Algébrico sem ruído. 


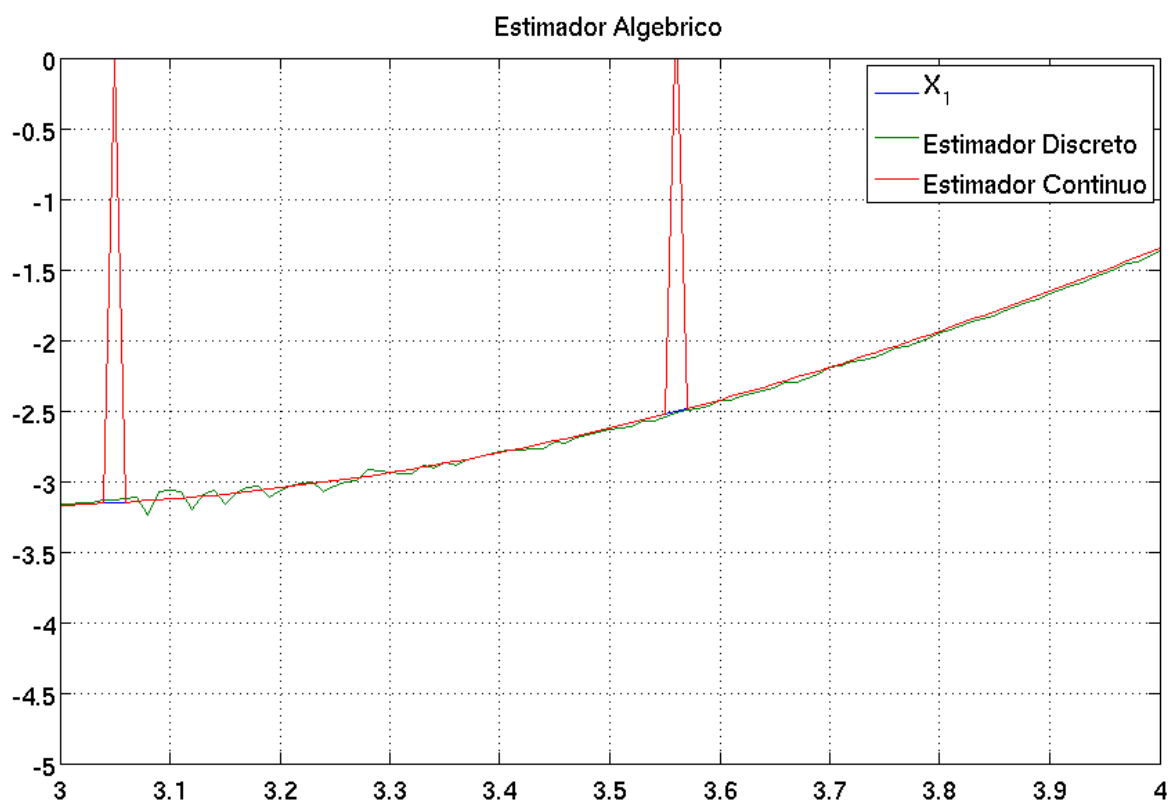

Figura 3.5: Detalhe do Estimador Algébrico sem ruído.

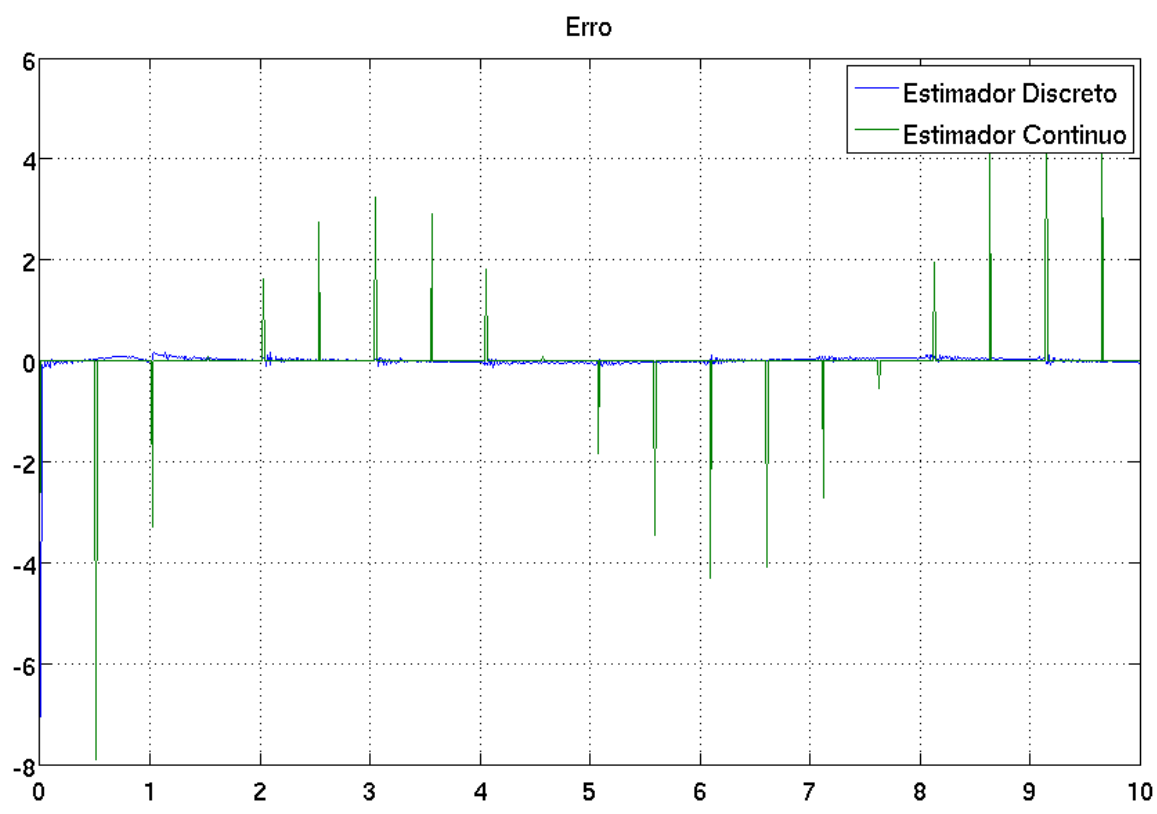

Figura 3.6: Erro no Estimador Algébrico sem ruído. 


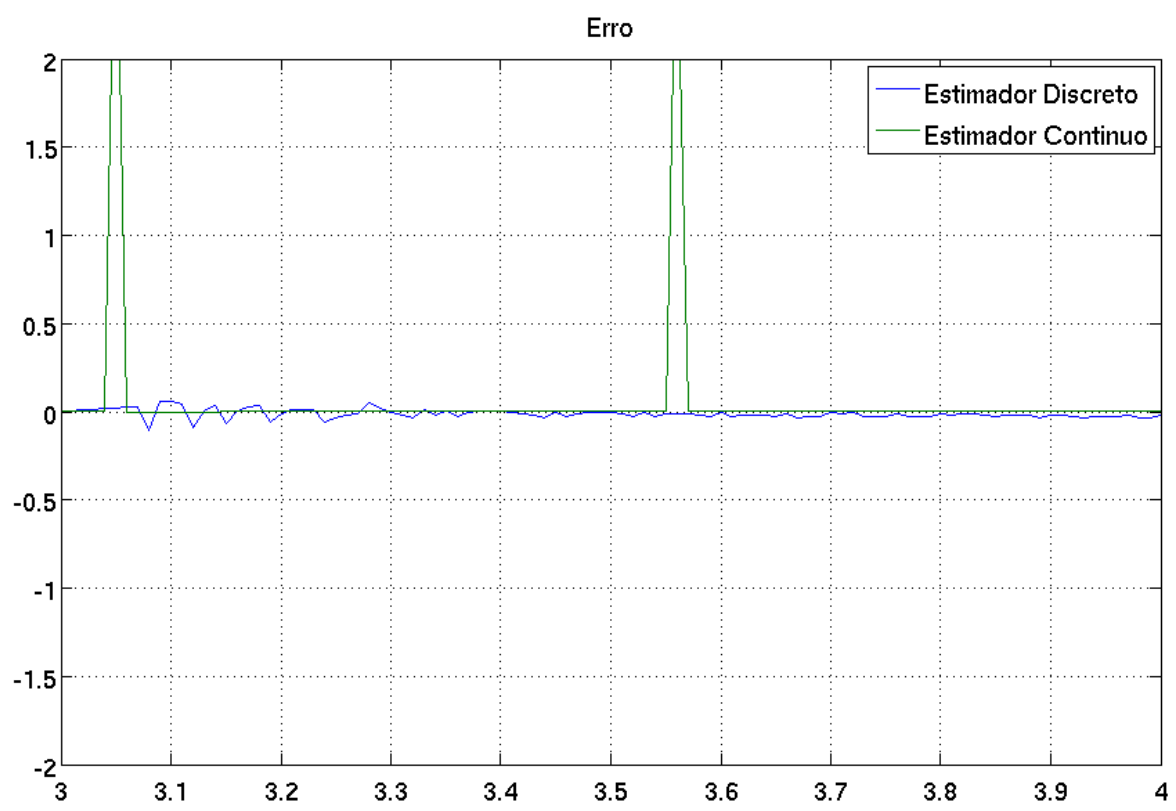

Figura 3.7: Detalhe do erro no Estimador Algébrico sem ruído.

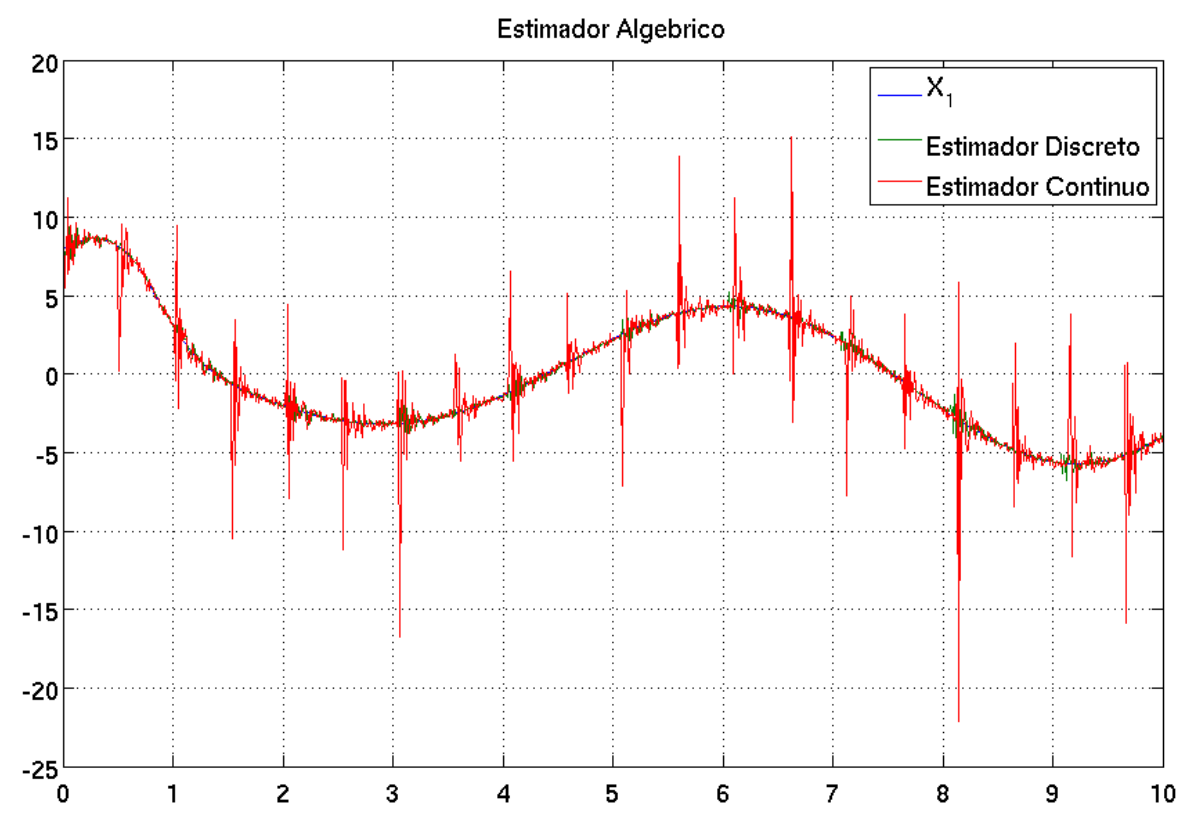

Figura 3.8: Estimador Algébrico com ruído. 


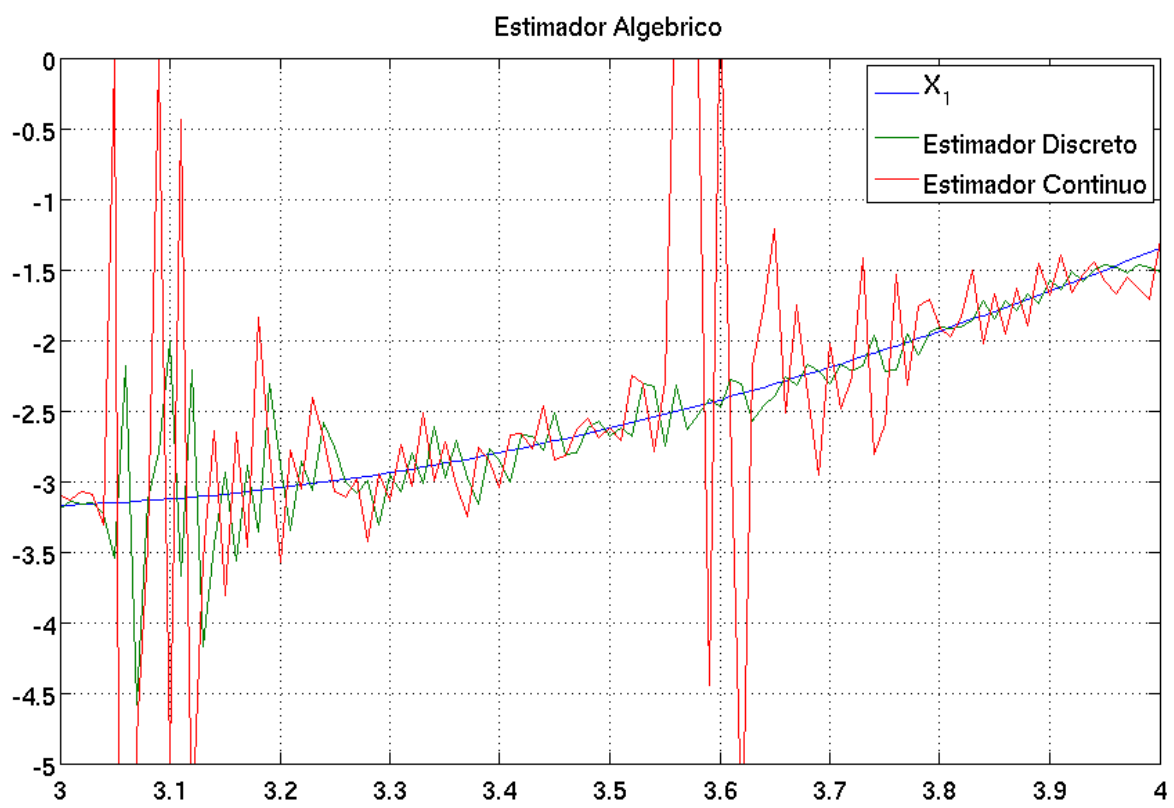

Figura 3.9: Detalhe do Estimador Algébrico com ruído.

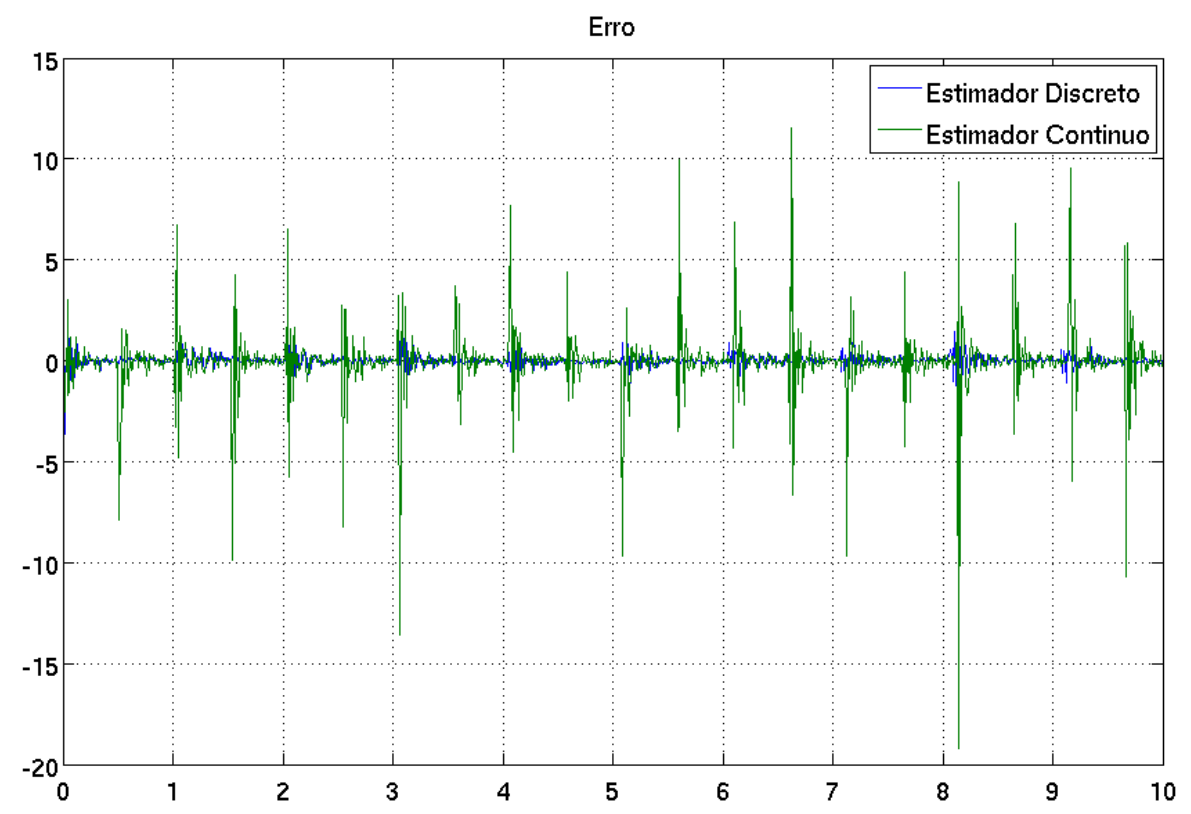

Figura 3.10: Erro no Estimador Algébrico com ruído. 


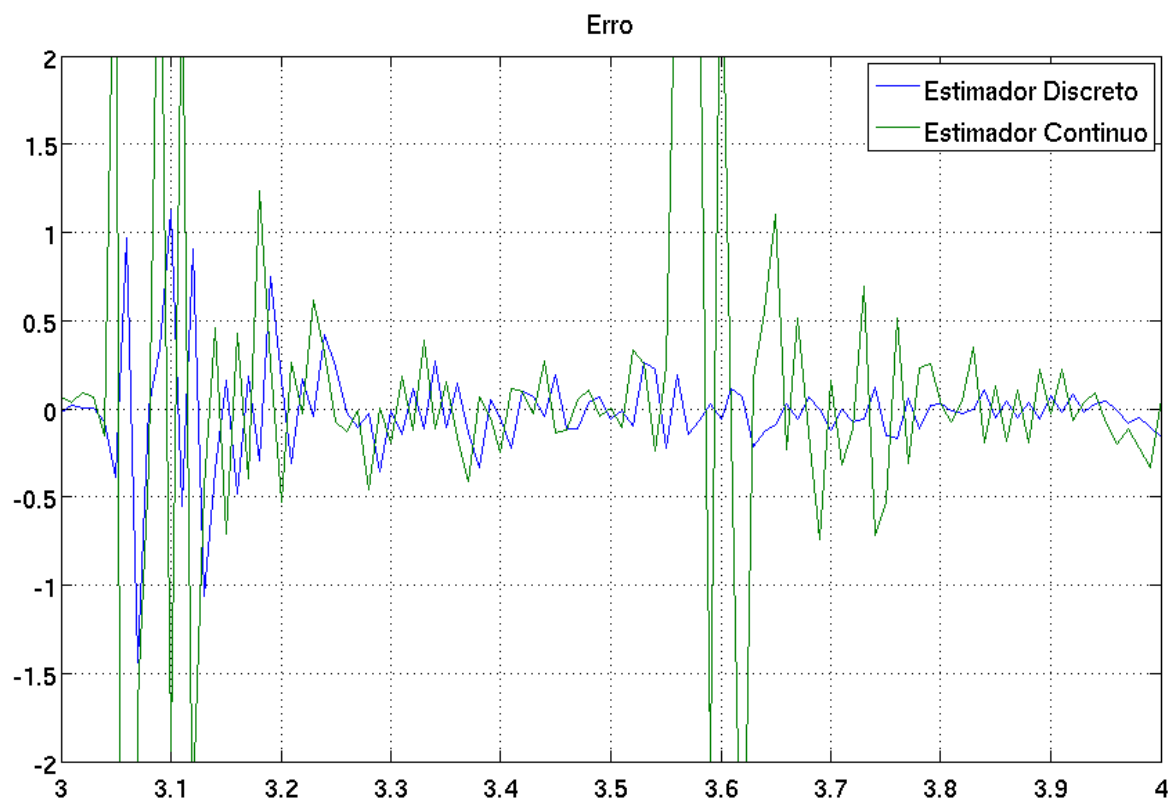

Figura 3.11: Detalhe do erro no Estimador Algébrico com ruído.

Observa-se que os resultados foram satisfatórios já que o estado foi estimado rápidamente e acompanhou muito bem o estado real. De um certo modo, observa-se que existe um delay de um intervalo de amostragem entre o estimador em tempo contínuo e nossa proposta para implementação em tempo discreto. Nas simulações utilizando o estimador em tempo discreto utilizamos dois estimadores em paralelo, conforme implementação descrita em [2]. Isto fez com que as estimativas em tempo discreto apresentassem um melhor comportamento em relação ao ruído.

Em ambiente livre de ruído o estimador discreto apresenta uma pequena oscilação, mas acompanha de maneira muito boa o resultado obtido pelo estimador em tempo contínuo. Já na presença de ruído, não se pode julgar qual estimador se comportou melhor, ambos apresentaram resultados bons e sem diferenças consideráveis. Deve-se recordar que o estimador em tempo discreto pode ser realizado na prática e com uma baixa taxa de amostragem, diferentemente do estimador em tempo contínuo que só pode ser simulado em ambiente computacional.

\subsection{Breve aplicação em controle}

Vamos descrever agora, um pequeno sistema não linear, encontrar uma lei de controle e observar o comportamento desta lei quando se faz uso dos estados estimados.

O sistema utilizado neste breve ensaio é descrito pelas equações a seguir: 


$$
\begin{aligned}
& \dot{x_{1}}=u+2 x_{1} \\
& \dot{x_{2}}=-x_{1}-x_{2}^{3} \\
& y_{1}=x_{1}^{2} \\
& y_{2}=x_{2}-x_{1}^{2}
\end{aligned}
$$

Trata-se de um sistema não linear de uma entrada $u$, duas saídas $\left(\begin{array}{l}y_{1} \\ y_{2}\end{array}\right)$ e dois estados internos $\left(\begin{array}{c}x_{1} \\ x_{2}\end{array}\right)$. Este sistema pode ser estabilizado por Lyapunov, se fizermos;

$$
\begin{aligned}
& V=x_{1}^{2}+x_{2}^{2}, \text { definida positiva. } \\
& \dot{V}=2 x_{1} \dot{x_{1}}+2 x_{2} \dot{x_{2}} \\
& \dot{V}=2 x_{1}\left(u+2 x_{1}\right)+2 x_{2}\left(-x_{1}-x_{2}^{3}\right) \\
& \dot{V}=2 x_{1} u+4 x_{1}^{2}-2 x_{2} x_{1}-2 x_{2}^{4}
\end{aligned}
$$

Escolhendo $u=-3 x_{1}+x_{2}$

$$
\begin{aligned}
& \dot{V}=2 x_{1}\left(-3 x_{1}+x_{2}\right)+4 x_{1}^{2}-2 x_{2} x_{1}-2 x_{2}^{4} \\
& \dot{V}=-6 x_{1}^{2}+2 x_{1} x_{2}+4 x_{1}^{2}-2 x_{2} x_{1}-2 x_{2}^{4} \\
& \dot{V}=-2 x_{1}^{2}-2 x_{2}^{4}, \text { definida negativa. }
\end{aligned}
$$

Portanto se fizermos $u=-3 x_{1}+x_{2}$, o sistema será globalmente e assintoticamente estável. A seguir apresentamos o comportamento obtido via simulação, figuras 3.12 e 3.13 para o sistema proposto quando partindo do estado interno definido por $x=\left(\begin{array}{c}2 \\ -2\end{array}\right)$ : 


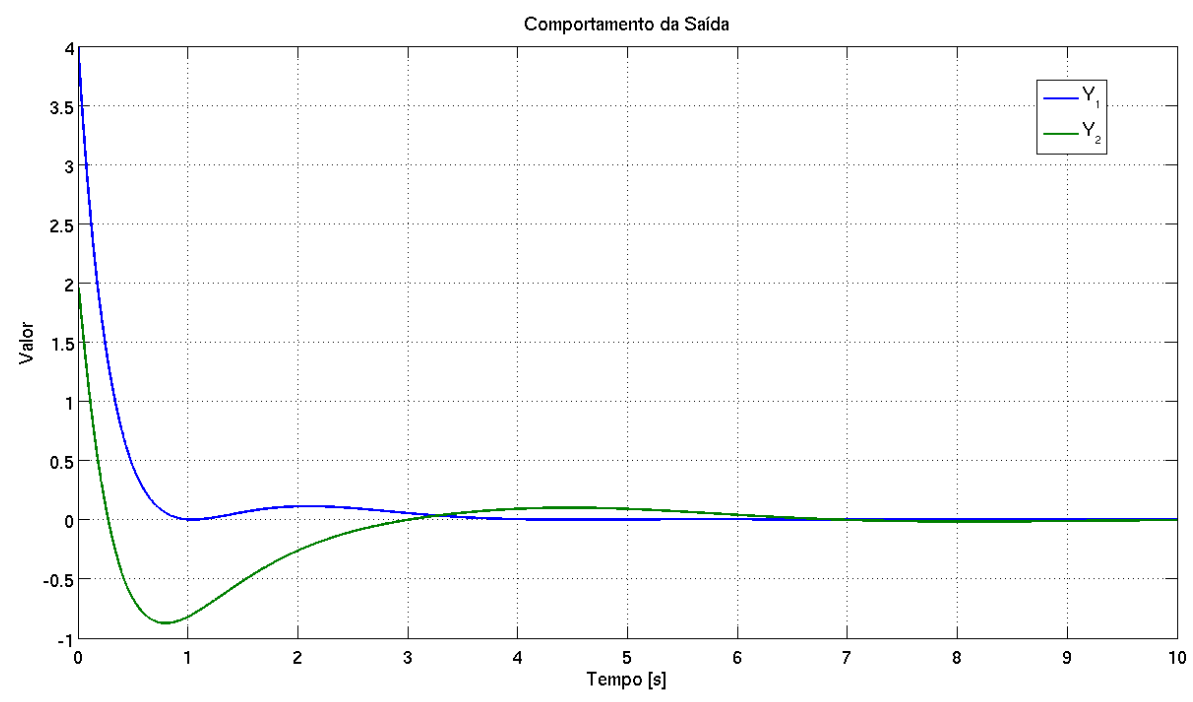

Figura 3.12: Comportamento da saída.

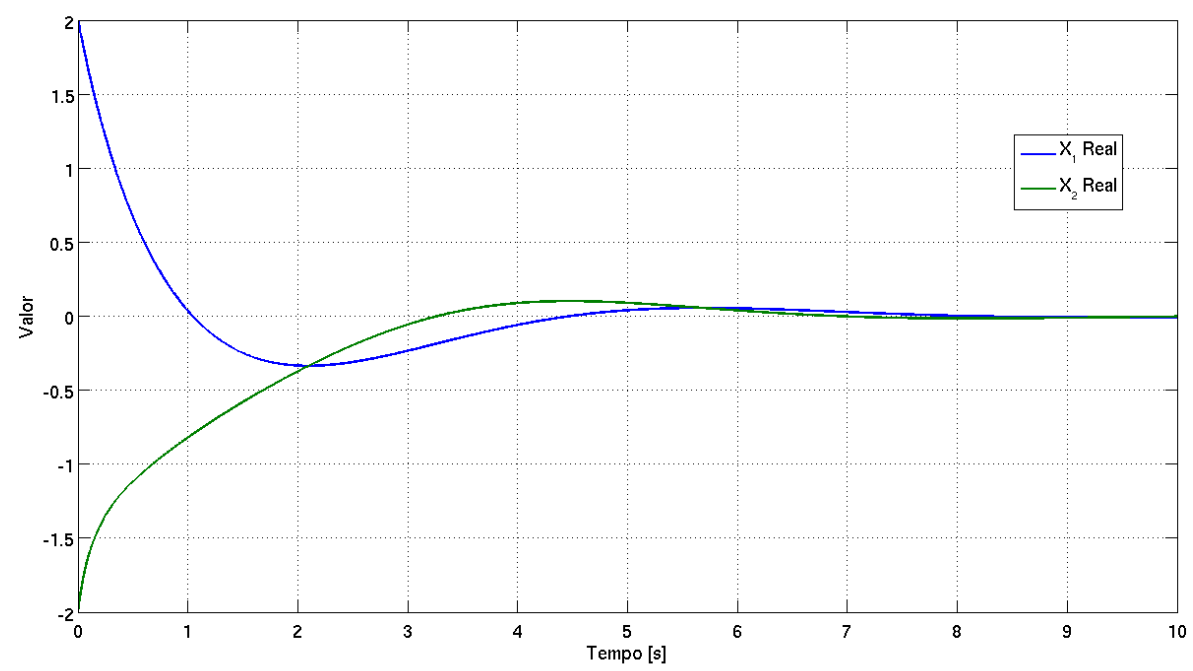

Figura 3.13: Comportamento dos estados internos

É possível obter os estados internos a partir das saídas e de suas derivadas. Para isto, basta observar que:

$x_{2}=y_{2}-y_{1}$

então:

$\dot{x_{2}}=\dot{y_{2}}-\dot{y_{1}}$

mas:

$\dot{x_{2}}=-x_{1}-x_{2}^{3}$ 
assim:

$$
\begin{aligned}
& -x_{1}-x_{2}^{3}=\dot{y_{2}}-\dot{y_{1}} \\
& -x_{1}=x_{2}^{3}+\dot{y_{2}}-\dot{y_{1}} \\
& -x_{1}=\left(y_{2}-y_{1}\right)^{3}+\dot{y}_{2}-\dot{y_{1}} \\
& x_{1}=-\left(y_{2}-y_{1}\right)^{3}-\dot{y_{2}}+\dot{y_{1}}
\end{aligned}
$$

Nas figuras 3.14 e 3.15, exibimos o comportamento do estimador em ambiente livre de ruído e quando sujeito a um ruído aditivo:

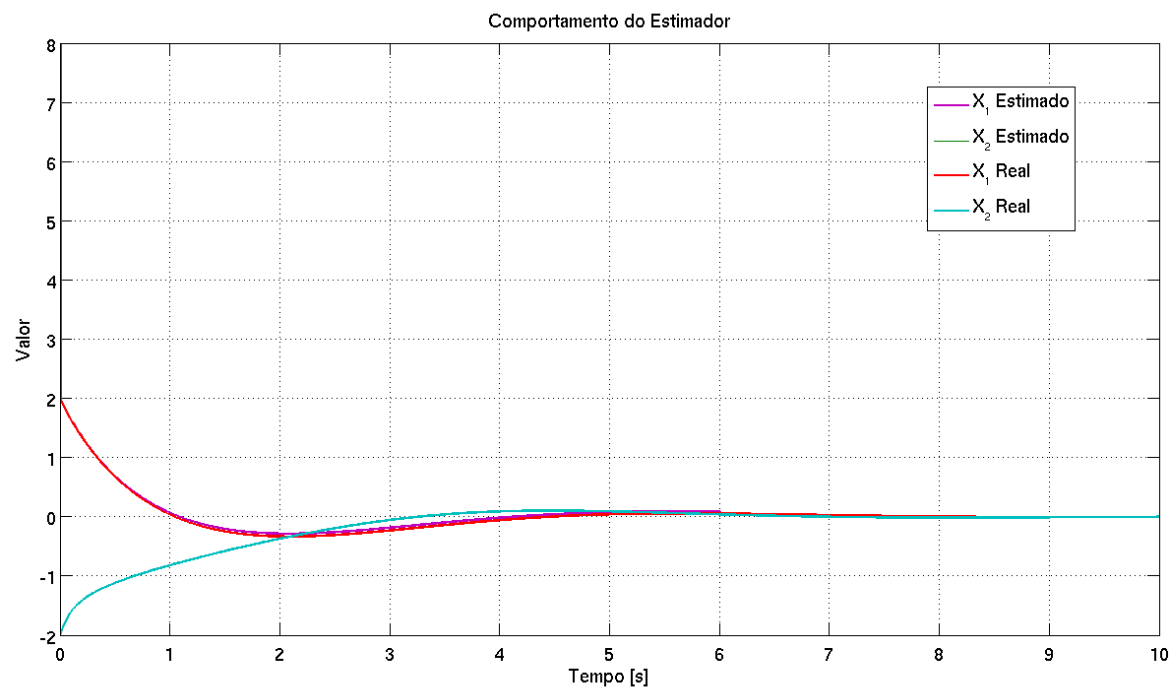

Figura 3.14: Comportamento do estimador.

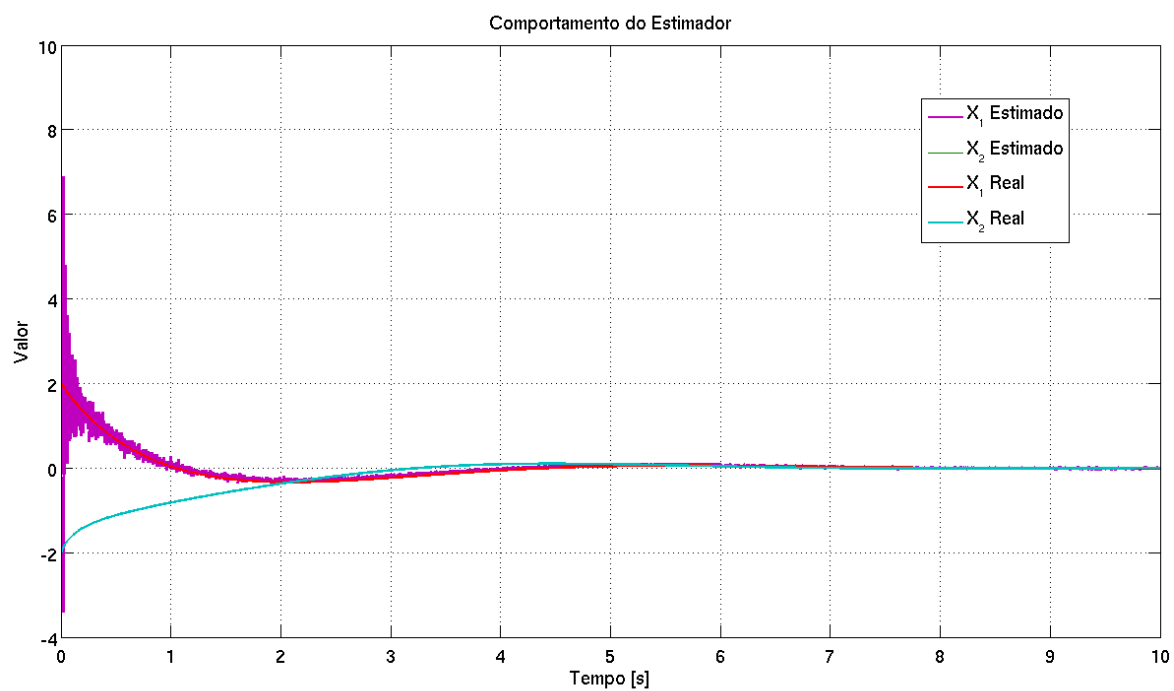

Figura 3.15: Comportamento do estimador na presença de ruído. 
Por fim, nas figuras 3.16, 3.18, 3.17 e 3.19, exibimos o comportamento do sistema quando a lei de controle faz uso dos estados estimados através das saídas:

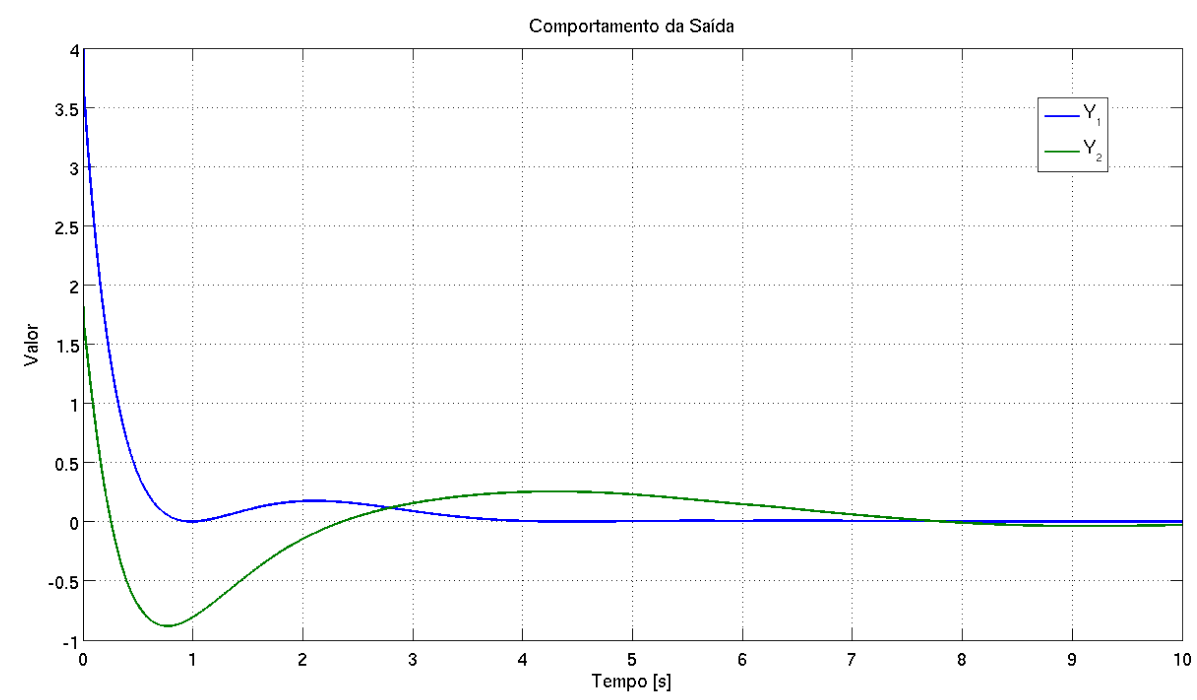

Figura 3.16: Comportamento da saída.

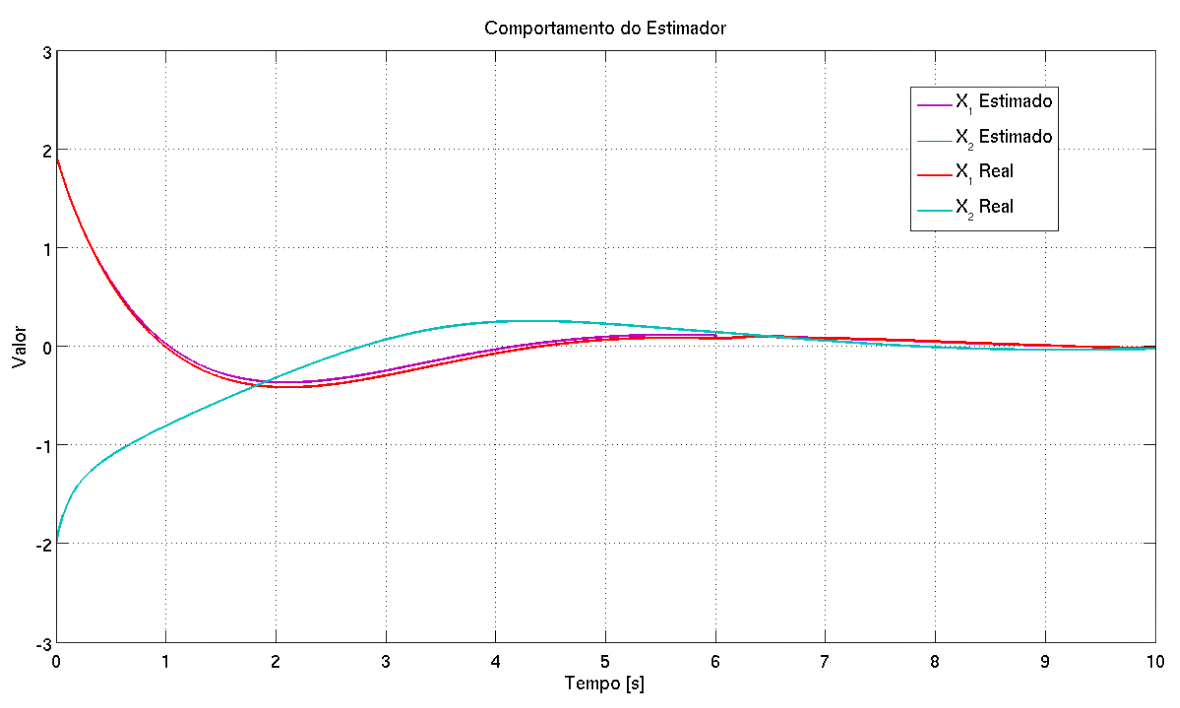

Figura 3.17: Comportamento do estimador. 


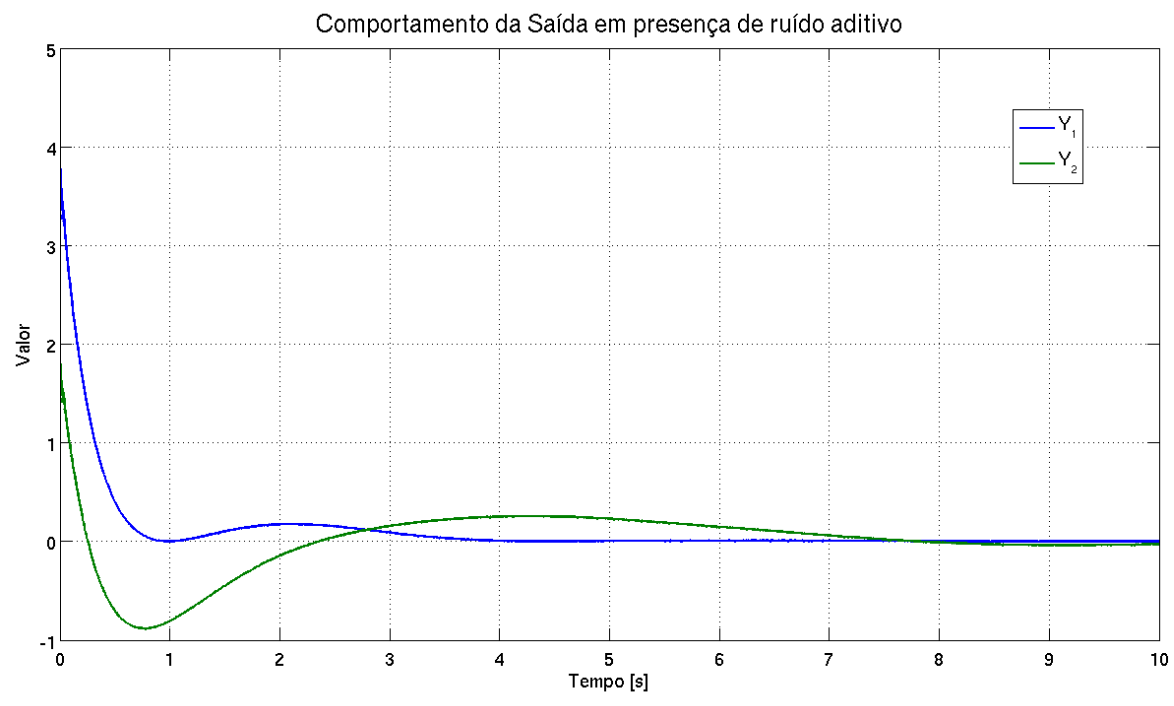

Figura 3.18: Comportamento da saída quando o estimador é perturbado por ruído.

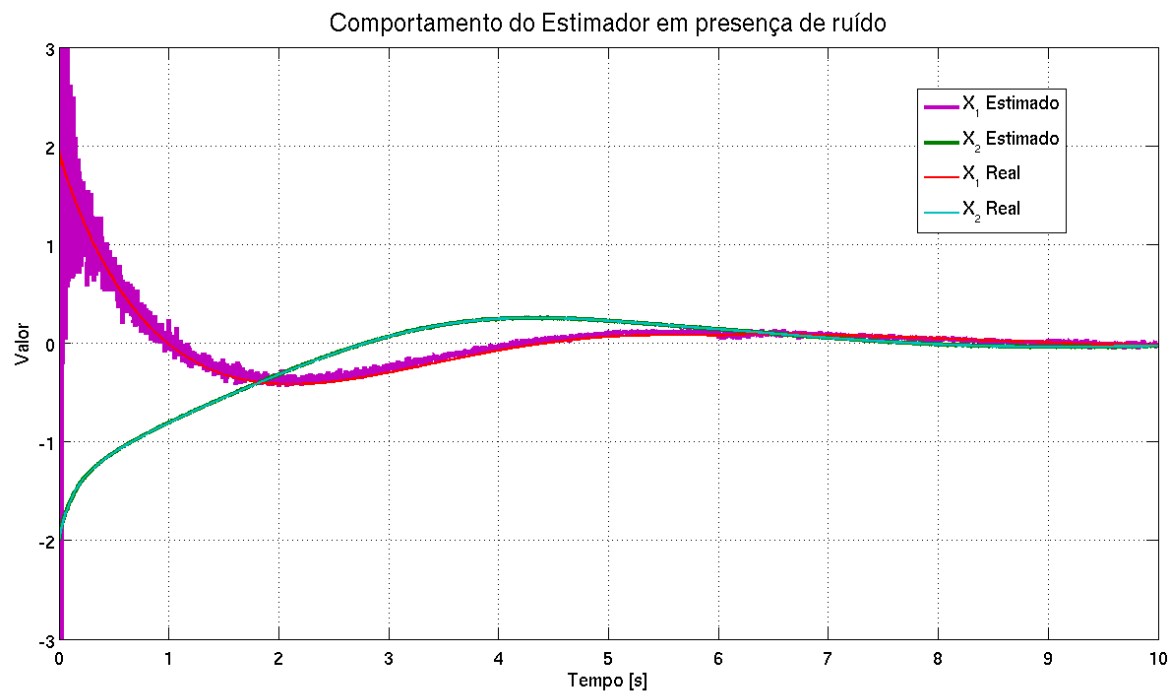

Figura 3.19: Comportamento do estimador quando perturbado por ruído.

Podemos observar que a realimentação dos estados estimados realmente se aproxima muito da realimentação de estados reais e que o ruído é atenuado com o passar do tempo.

\subsection{Possibilidades não exploradas}

A técnica abordada em [3] e detalhada na seção 3.2 é apenas uma maneira encontrada para realizar o filtro da equação (2.22) em tempo discreto. Outras possibilidades podem levar a resultados melhores ou piores, mas não foram exploradas no presente trabalho. 
Em especial, sabe-se que aproximar por um polinômio um sinal com sequiências de amostras com valor constante não gera bons resultados. O polinômio aproximador tende a gerar ondulações que originalmente não existiam e isto compromete o comportamento do estimador. Uma possível extensão do presente trabalho seria estudar o comportamento de outras técnicas de representação do sinal amostrado, aproximando-o por um "spline" por exemplo. Obviamente alguns detalhes de cálculo deverão ser modificados, mas possivelmente se produzam estimativas ainda mais robustas. 


\section{Sistema Mecânico}

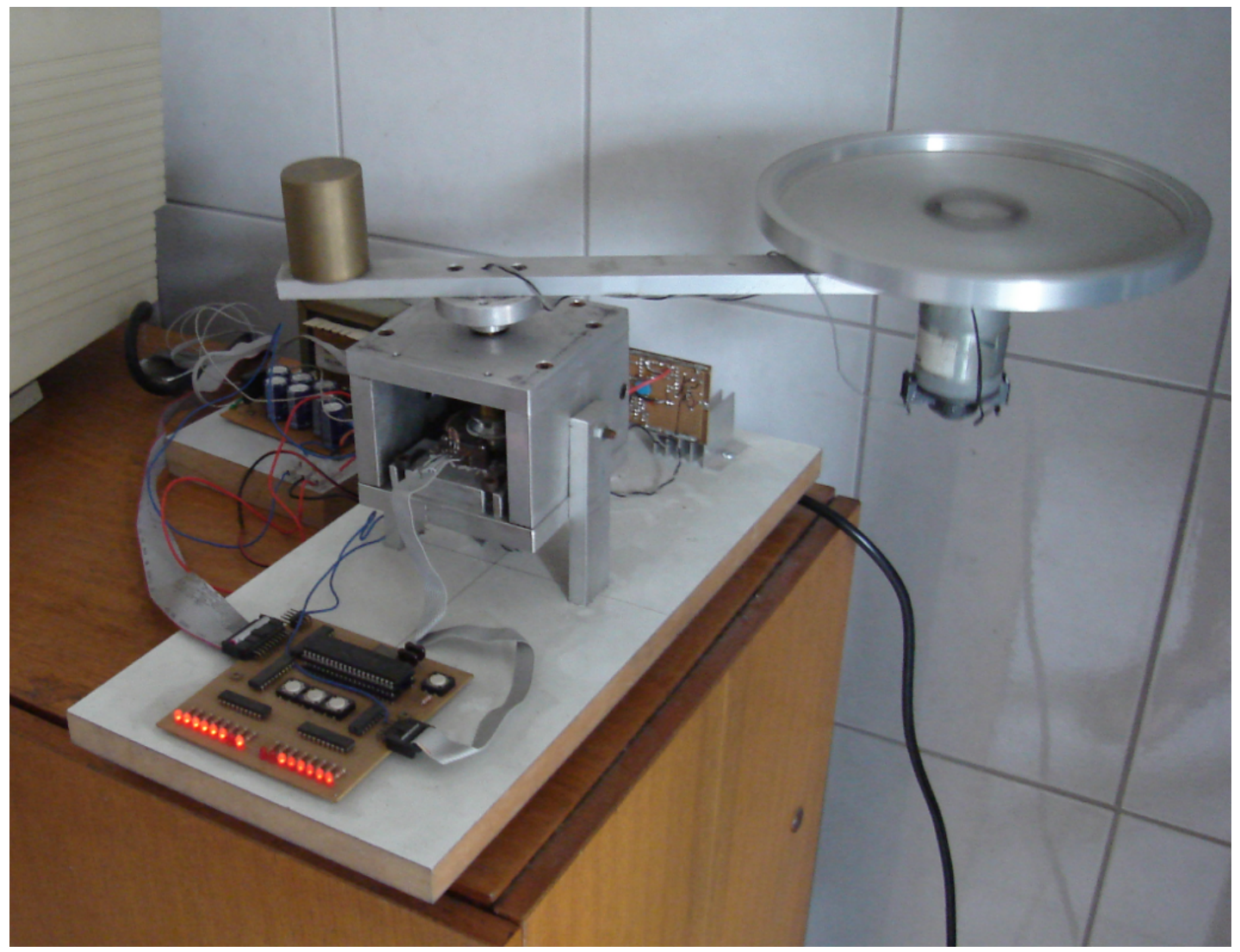

Neste capítulo vamos descrever o sistema mecânico construído e elaborar o seu modelo representativo. Um modelo de atrito é apresentado e após alguns ensaios identificamos os parâmetros pertinentes de atrito. 


\subsection{Descrição do sistema}

O pêndulo invertido via transferência de torque que será analisado, equacionado e simulado neste trabalho é composto basicamente por uma barra que pode girar livremente em torno de um eixo fixo. Em uma das extremidades da barra e fixo a esta, existe um motor de corrente contínua. Preso ao eixo do motor está um disco. Na outra extremidade da barra temos um contra-peso.

A barra gira sem sair do plano perpendicular ao eixo fixo, que por sua vez pode estar em qualquer posição e formando ângulos diversos com o plano horizontal. Na figura 4.1 temos uma ilustração do sistema onde o plano da barra é perpendicular ao plano horizontal, situação em que se exige o maior esforço do motor para obter o equilíbrio não trivial.

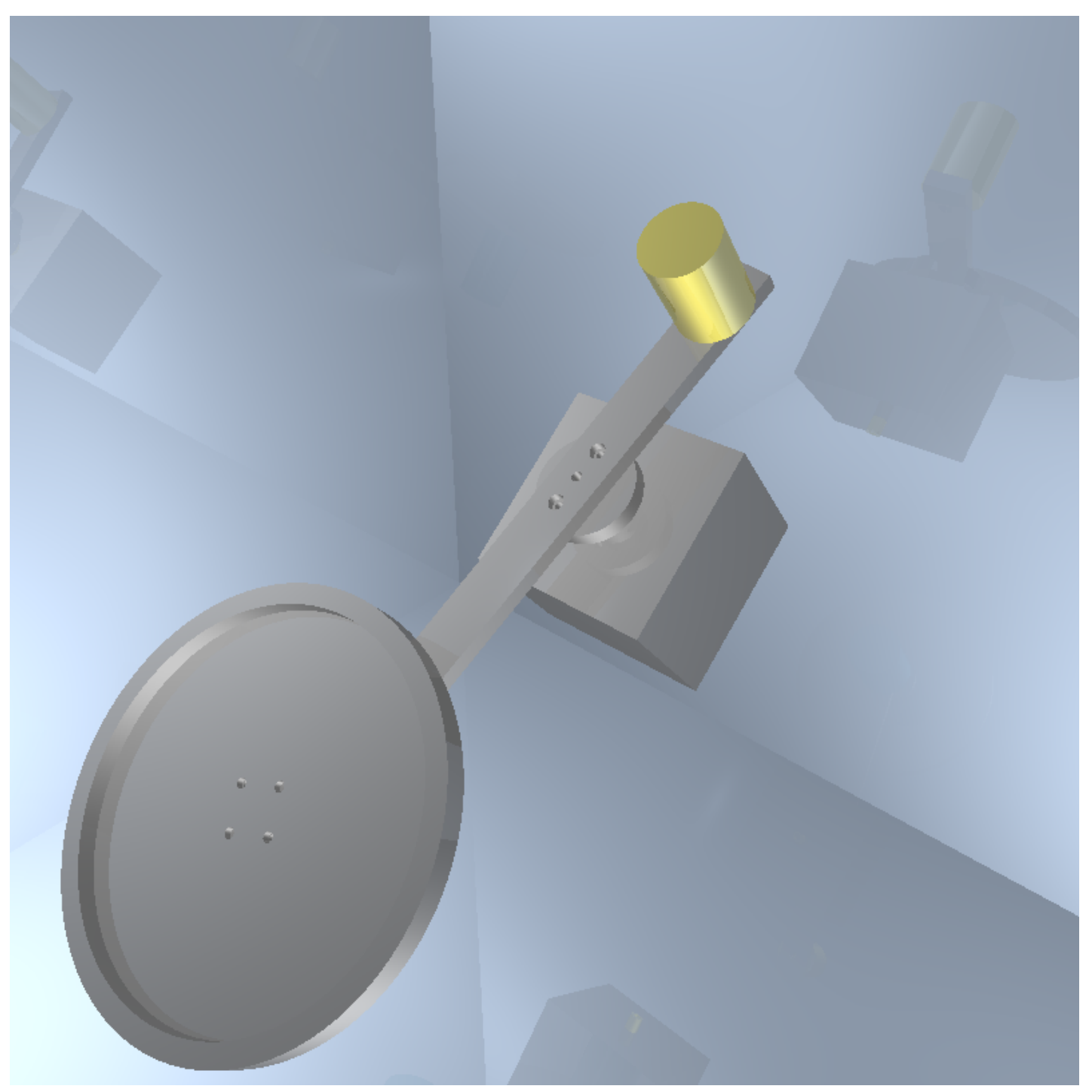

Figura 4.1: Visão geral do pêndulo. 
Ao se aplicar uma corrente no motor, temos um torque aplicado ao disco e como reação, um torque é aplicado ao motor e à barra, transferindo movimento a esta. Na situação ilustrada pela figura 4.1, tem-se intuitivamente dois pontos de equilíbrio, um estável e outro instável.

O sistema em questão é não linear, devido à inclinação da barra modificar o torque através de uma função seno. Apesar de ser simples, o comportamento deste sistema nem sempre é intuitivo.

\subsection{Modelagem do sistema}

\subsubsection{Definição da simbologia adotada}

Para proceder a modelagem do sistema, cabe definir e entender o sistema de coordenadas adotado, bem como a nomenclatura das constantes utilizadas.

As coordenadas fundamentais do sistema serão definidas, deste ponto em diante, como:

\begin{tabular}{|c|c|}
\hline Símbolo & Descrição \\
\hline \hline$\phi$ & $\begin{array}{c}\text { Ângulo formado entre a barra e o plano vertical, nulo quando } \\
\text { em equilíbrio não trivial, para } 0<\eta<2 \pi\end{array}$ \\
\hline$\theta$ & Ângulo formado entre um ponto de referência no disco e a barra. \\
\hline$\eta$ & $\begin{array}{c}\text { Ângulo formado entre o plano da barra e o plano horizontal. } \\
\text { Constante durante os ensaios. }\end{array}$ \\
\hline
\end{tabular}

Tabela 4.1: Coordenadas Utilizadas.

Para tornar mais claro o equacionamento do sistema escolhido, as coordenadas estão explicitadas na figura 4.2. 


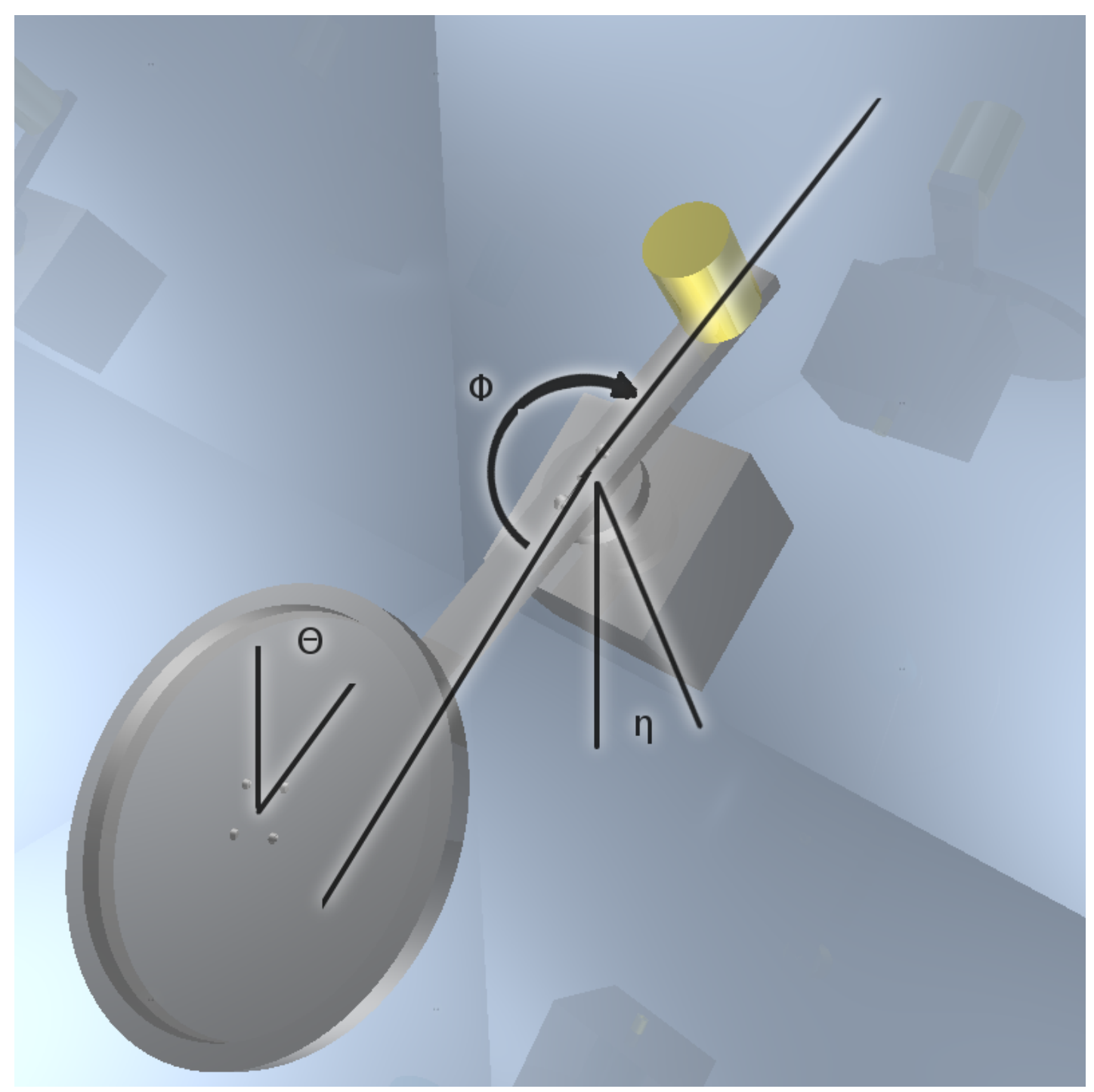

Figura 4.2: Ilustração das Coordenadas Utilizadas.

As constantes serão definidas utilizando a nomenclatura Grandeza $a_{\text {Objeto }}$, identificando a natureza da grandeza física e em sub-escrito o objeto ou parte do sistema ao qual a grandeza física em questão diz respeito.

As representações que serão utilizadas encontram-se nas tabelas 4.2 e 4.3:

\begin{tabular}{|c||c|}
\hline Simbolo de Grandeza & Descrição \\
\hline \hline$h$ & Altura \\
\hline$M$ & Massa \\
\hline$L$ & Comprimento \\
\hline$\tau$ & Torque \\
\hline$J$ & Momento de Inercia \\
\hline$\tau_{a t,}$ & Torque de Atrito \\
\hline
\end{tabular}

Tabela 4.2: Simbologia das grandezas físicas. 


\begin{tabular}{|c||c|}
\hline Simbolo de parte ou objeto & Descrição \\
\hline$M O T$ & Motor \\
\hline$D I S$ & Disco \\
\hline$C I L$ & Cilindro de Contra Peso \\
\hline$B A R$ & Barra em sua totalidade \\
\hline$B A R 1$ & Trecho da barra que sustenta o motor e disco \\
\hline$B A R 2$ & Trecho da barra que sustenta o contra peso \\
\hline
\end{tabular}

Tabela 4.3: Simbologia dos componentes do sistema.

Desta maneira, $M_{C I L}$ por exemplo, faz referência à massa do cilindro compensador de peso.

\subsubsection{Modelagem do sistema através do formalismo Lagrangiano}

\section{Energia potencial}

A energia potencial do sistema é dada pela soma das energias potenciais de cada elemento que o constitui, assim:

$$
\begin{aligned}
& V=V_{C I L}+V_{B A R 1}+V_{B A R 2}+V_{M O T}+V_{D I S} \\
& V=g\left(M_{C I L} h_{C I L}+M_{B A R 1} h_{B A R 1}+M_{B A R 2} h_{B A R 2}+M_{M O T} h_{M O T}+M_{D I S} h_{D I S}\right)
\end{aligned}
$$

Observa-se que as alturas dos centros de massa são:

$$
\begin{aligned}
& h_{C I L}=-L_{B A R 2} \sin (\eta) \cos (\phi) \\
& h_{M O T}=h_{D I S}=L_{B A R 1} \sin (\eta) \cos (\phi) \\
& h_{B A R 1}=\frac{L_{B A R 1}}{2} \sin (\eta) \cos (\phi) \\
& h_{B A R 2}=-\frac{L_{B A R 2}}{2} \sin (\eta) \cos (\phi)
\end{aligned}
$$

Efetuando os cálculos temos:

$$
\begin{aligned}
& V=g \sin (\eta) \cos (\phi)\left(M_{M O T} L_{B A R 1}+M_{D I S} L_{B A R 1}+M_{B A R 1} \frac{L_{B A R 1}}{2}-M_{C I L} L_{B A R 2}-M_{B A R 2} \frac{L_{B A R 2}}{2}\right) \\
& V=g \sin (\eta) \cos (\phi)\left(L_{B A R 1}\left(\frac{M_{B A R 1}}{2}+M_{M O T}+M_{D I S}\right)-L_{B A R 2}\left(M_{C I L}+\frac{M_{B A R 2}}{2}\right)\right)
\end{aligned}
$$

Chamando:

$\mathbb{C}_{3}=g \sin (\eta)\left(L_{B A R 1}\left(\frac{M_{B A R 1}}{2}+M_{M O T}+M_{D I S}\right)-L_{B A R 2}\left(M_{C I L}+\frac{M_{B A R 2}}{2}\right)\right)$

Então:

$V=\mathbb{C}_{3} \cos (\phi)$ 


\section{Energia cinética}

A energia cinética do sistema é dada pela soma das energias cinéticas de cada elemento, assim:

$$
T=T_{C I L}+T_{B A R 1}+T_{B A R 2}+T_{M O T}+T_{D I S}
$$

$$
T=\frac{J_{C I L}}{2} \dot{\phi}^{2}+\frac{J_{B A R 1}}{2} \dot{\phi}^{2}+\frac{J_{B A R 2}}{2} \dot{\phi}^{2}+\frac{J_{M O T}}{2} \dot{\phi}^{2}+\frac{J_{D I S}}{2}(\dot{\theta}+\dot{\phi})^{2}+\frac{M_{D I S}}{2}\left(\dot{\phi} L_{B A R 1}\right)^{2}
$$

$$
T=\frac{J_{C I L}+J_{B A R 1}+J_{B A R 2}+J_{M O T}+L_{B A R 1}^{2} M_{D I S}}{2} \dot{\phi}^{2}+\frac{J_{D I S}}{2}(\dot{\theta}+\dot{\phi})^{2}
$$

Chamando:

$$
\begin{gathered}
\mathbb{C}_{1}=\frac{1}{J_{C I L}+J_{B A R 1}+J_{B A R 2}+J_{M O T}+L_{B A R 1}^{2} M_{D I S}} \\
\mathbb{C}_{2}=\frac{1}{J_{D I S}}
\end{gathered}
$$

Então:

$$
T=\frac{\dot{\phi}^{2}}{2 \mathbb{C}_{1}}+\frac{(\dot{\theta}+\dot{\phi})^{2}}{2 \mathbb{C}_{2}}
$$

\section{Lagrangiana}

$$
\mathscr{L}=T-V=\frac{\dot{\phi}^{2}}{2 \mathbb{C}_{1}}+\frac{(\dot{\theta}+\dot{\phi})^{2}}{2 \mathbb{C}_{2}}-\mathbb{C}_{3} \cos (\phi)
$$

Definindo as coordenadas generalizadas

$$
q_{1}=\theta
$$




$$
q_{2}=\phi
$$

$\mathrm{E}$ as forças generalizadas

$$
\begin{gathered}
Q_{1}=\tau_{M O T}+\tau_{a t, D I S} \\
Q_{2}=\tau_{a t, B A R} \\
\mathscr{L}=\frac{\dot{q}_{2}^{2}}{2 \mathbb{C}_{1}}+\frac{\left(\dot{q}_{1}+\dot{q}_{2}\right)^{2}}{2 \mathbb{C}_{2}}-\mathbb{C}_{3} \cos \left(q_{2}\right)
\end{gathered}
$$

Equação de Lagrange para a variável generalizada $q_{1}=\theta$

$$
\begin{gathered}
\frac{\partial \mathscr{L}}{\partial \dot{q}_{1}}=\frac{\dot{q}_{1}}{\mathbb{C}_{2}}+\frac{\dot{q}_{2}}{\mathbb{C}_{2}} \\
\frac{d}{d t}\left(\frac{\partial \mathscr{L}}{\partial \dot{q}_{1}}\right)=\frac{\ddot{q}_{1}}{\mathbb{C}_{2}}+\frac{\ddot{q}_{2}}{\mathbb{C}_{2}} \\
\frac{\partial \mathscr{L}}{\partial q_{1}}=0
\end{gathered}
$$

Então a equação de Lagrange fica:

$$
\frac{\ddot{q}_{1}}{\mathbb{C}_{2}}+\frac{\ddot{q}_{2}}{\mathbb{C}_{2}}=Q_{1}
$$

Equação de Lagrange para a variável generalizada $q_{2}=\phi$

$$
\frac{\partial \mathscr{L}}{\partial \dot{q}_{2}}=\frac{\dot{q}_{1}}{\mathbb{C}_{2}}+\frac{\dot{q}_{2}}{\mathbb{C}_{2}}+\frac{\dot{q}_{2}}{\mathbb{C}_{1}}
$$




$$
\begin{gathered}
\frac{d}{d t}\left(\frac{\partial \mathscr{L}}{\partial \dot{q}_{2}}\right)=\frac{\ddot{q}_{1}}{\mathbb{C}_{2}}+\frac{\ddot{q}_{2}}{\mathbb{C}_{2}}+\frac{\ddot{q}_{2}}{\mathbb{C}_{1}} \\
\frac{\partial \mathscr{L}}{\partial \phi}=\mathbb{C}_{3} \sin \left(q_{2}\right)
\end{gathered}
$$

Então, a equação de Lagrange fica:

$$
\frac{\ddot{q}_{1}}{\mathbb{C}_{2}}+\frac{\ddot{q}_{2}}{\mathbb{C}_{2}}+\frac{\ddot{q}_{2}}{\mathbb{C}_{1}}-\mathbb{C}_{3} \sin \left(q_{2}\right)=Q_{2}
$$

\section{Equações desacopladas}

Isolando $\frac{\ddot{q}_{1}}{\mathbb{C}_{2}}$ da equação (4.16):

$$
\frac{\ddot{q}_{1}}{\mathbb{C}_{2}}=Q_{1}-\frac{\ddot{q}_{2}}{\mathbb{C}_{2}}
$$

e substituindo na equação (4.20), isolamos $\ddot{q}_{2}$ :

$$
\begin{gathered}
Q_{1}-\frac{\ddot{q}_{2}}{\mathbb{C}_{2}}+\frac{\ddot{q}_{2}}{\mathbb{C}_{2}}+\frac{\ddot{q}_{2}}{\mathbb{C}_{1}}-\mathbb{C}_{3} \sin \left(q_{2}\right)=Q_{2} \\
\ddot{q}_{2}=\mathbb{C}_{1} Q_{2}-\mathbb{C}_{1} Q_{1}+\mathbb{C}_{1} \mathbb{C}_{3} \sin \left(q_{2}\right)
\end{gathered}
$$

E em seguida, substituindo na equação (4.16):

$$
\begin{aligned}
& \frac{\ddot{q}_{1}}{\mathbb{C}_{2}}+\frac{\mathbb{C}_{1} Q_{2}-\mathbb{C}_{1} Q_{1}+\mathbb{C}_{1} \mathbb{C}_{3} \sin \left(q_{2}\right)}{\mathbb{C}_{2}}=Q_{1} \\
& \ddot{q}_{1}=\left(\mathbb{C}_{1}+\mathbb{C}_{2}\right) Q_{1}-\mathbb{C}_{1} Q_{2}-\mathbb{C}_{1} \mathbb{C}_{3} \sin \left(q_{2}\right)
\end{aligned}
$$

Por fim, as equações de movimento, são:

$$
\ddot{\theta}=\left(\mathbb{C}_{2}+\mathbb{C}_{1}\right) \tau_{M O T}+\left(\mathbb{C}_{2}+\mathbb{C}_{1}\right) \tau_{a t, D I S}-\mathbb{C}_{1} \mathbb{C}_{3} \sin (\phi)-\mathbb{C}_{1} \tau_{a t, B A R}
$$




$$
\ddot{\phi}=\mathbb{C}_{1} \mathbb{C}_{3} \sin (\phi)-\mathbb{C}_{1} \tau_{M O T}+\mathbb{C}_{1} \tau_{a t, B A R}-\mathbb{C}_{1} \tau_{a t, D I S}
$$

\subsubsection{Modelagem do atrito}

Modelar o atrito seco é uma tarefa um tanto complicada, uma vez que tal fenômeno exibe um comportamento com muitos detalhes e dependente de algumas condições do sistema. Um modelo mais representativo de atrito, como é o modelo de Coulomb, apresenta uma descontinuidade para velocidade nula, impedindo o uso de integração numérica nas simulações. No presente trabalho, adotaremos um modelo definido em [10], que aproxima o modelo de Coulomb, mas é continuamente diferenciável.

Tal modelo é descrito pela equação a seguir:

$$
F_{a t}(\dot{q})=\gamma_{1}\left(\tanh \left(\gamma_{2} \dot{q}\right)-\tanh \left(\gamma_{3} \dot{q}\right)\right)+\gamma_{4} \tanh \left(\gamma_{5} \dot{q}\right)+\gamma_{6} \dot{q}
$$

onde $\gamma_{i} \in \mathbb{R}^{+} \forall i=1,2, \ldots, 6$ são constantes positivas.

Os parâmetros $\gamma_{1}, \gamma_{2}$ e $\gamma_{3}$ são responsáveis pelo efeito Stribeck que modela o atrito estático, $\gamma_{4}$ e $\gamma_{5}$ determinam o comportamento do atrito cinético, e finalmente, $\gamma_{6}$ determina o fator linear diretamente proporcional à velocidade.

A figura 4.3 ilustra o modelo de atrito utilizado, para as seguintes constantes $\gamma=\left(\begin{array}{llllll}4 & 3 & 1 & \frac{1}{2} & 3 & \frac{1}{50}\end{array}\right)$ :

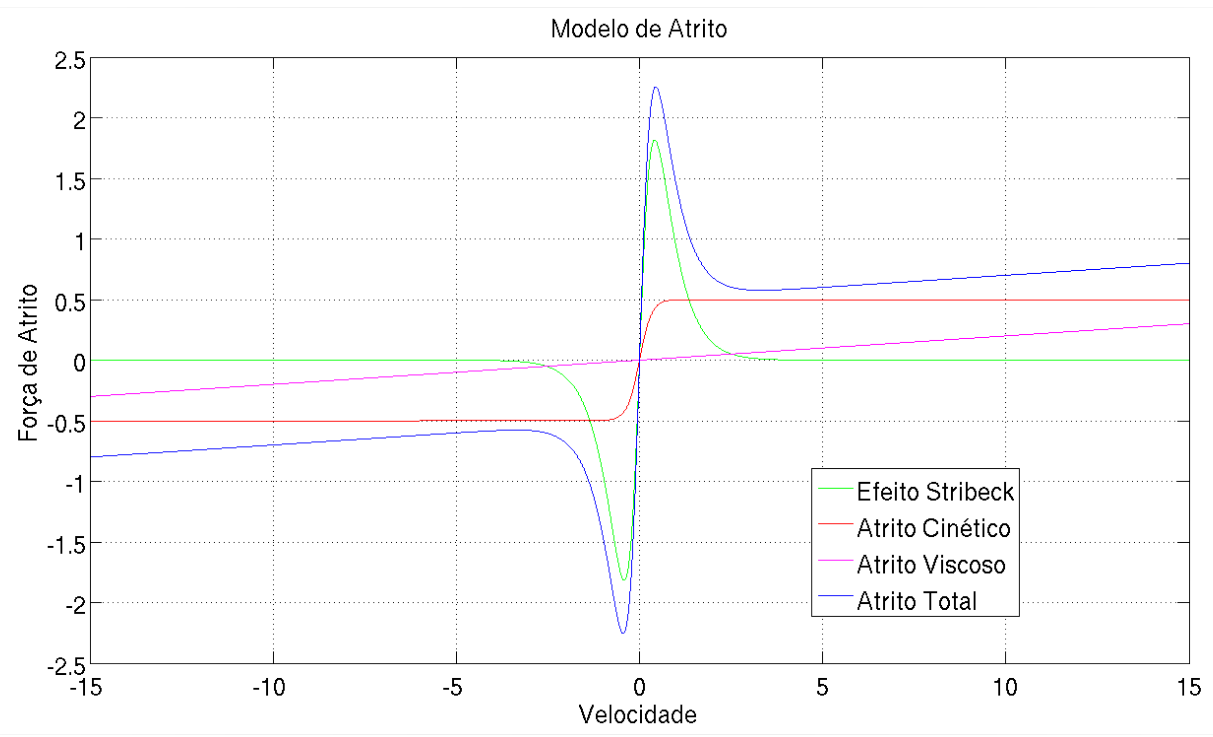

Figura 4.3: Gráfico de comportamento do modelo de atrito. 
Deve-se ressaltar que este modelo reproduz a força (ou torque) de atrito, representada com sentido contrário à velocidade. Na dedução do modelo do sistema mecânico, adotamos uma representação absoluta para todas as forças, momentos, velocidades e acelerações, de forma que o torque de atrito é representado com o mesmo sentido da velocidade e por este motivo, invertemos o sinal do torque nos modelos de atrito usados em conjunto com o modelo do sistema mecânico.

A obtenção das constantes a partir dos valores de atrito estático, cinético e viscoso é feita, inicialmente, de maneira aproximada. Esta aproximação é então refinada por um processo inspirado em simulações de recozimento (annealing simulated optimization) ${ }^{1}$ [11], de forma a encontrar parâmetros que minimizem a distância da curva resultante de alguns pontos desejáveis. Estes pontos são o máximo de atrito estático, o mínimo de atrito cinético, ambos de velocidade diminuta, e um ponto de velocidade elevada que deve aproximar o atrito cinético somado ao termo proporcional à velocidade.

$$
\text { O vetor de constantes } \gamma=\left(\begin{array}{llllll}
27.8864 & 133.6172 & 114.9599 & 0.2187 & 31.1378 & 0.0200
\end{array}\right)
$$
foi otimizado para gerar um coeficiente de atrito estático de $2 \mathrm{~N}$, um coeficiente de atrito cinético de $0,2 \mathrm{~N}$ e um atrito viscoso equivalente a velocidade multiplicada por 0,02 . O pico de atrito estático ocorre a $0,02 \frac{\mathrm{m}}{\mathrm{s}}$ e o vale de atrito cinético ocorre a $0,05 \frac{\mathrm{m}}{\mathrm{s}}$. Para este cenário de atrito, apresentamos o comportamento de um bloco de massa $1 \mathrm{Kg}$ em algumas situações distintas:

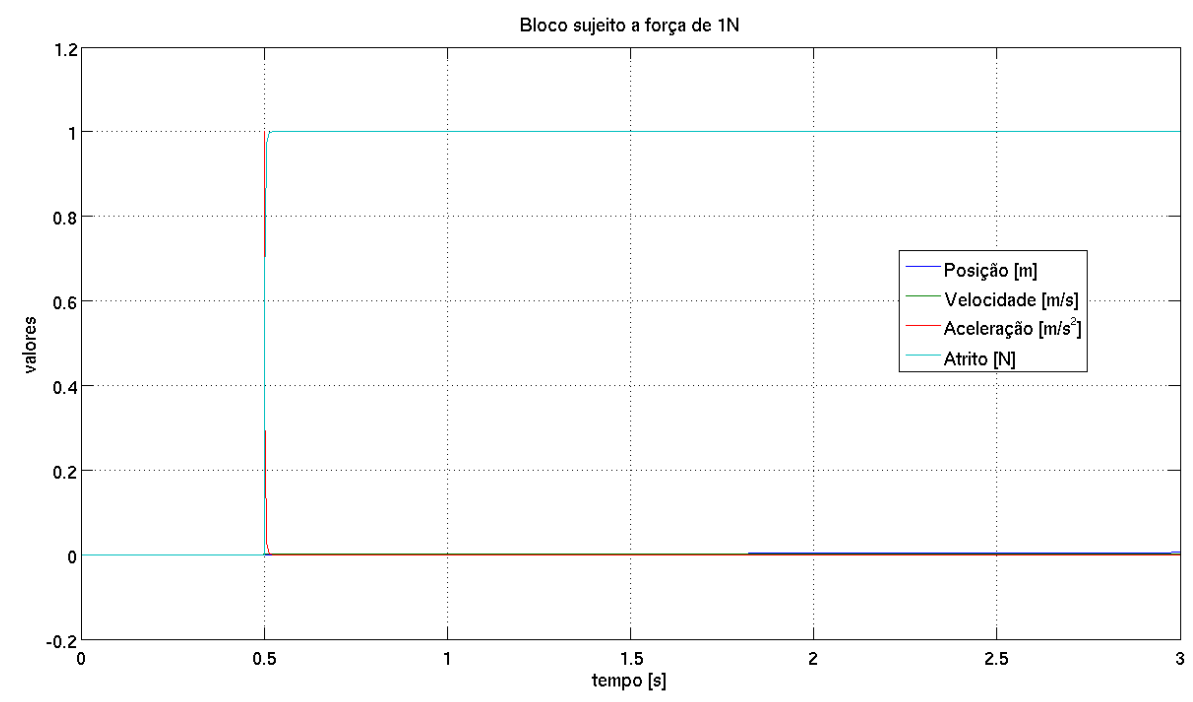

Figura 4.4: Ensaio do modelo de atrito, força de $1 \mathrm{~N}$ aplicada ao bloco

\footnotetext{
${ }^{1}$ As constantes são escolhidos de forma aleatória e se registra a cada teste o valor do grupo que ofereceu melhor aproximação segundo um índice previamente definido de qualidade. A cada novo teste os valores são escolhidos como pequenas flutuações do melhor grupo, a variância do termo aleatório é reduzida até zero quando se seleciona o melhor grupo obtido no ensaio.
} 


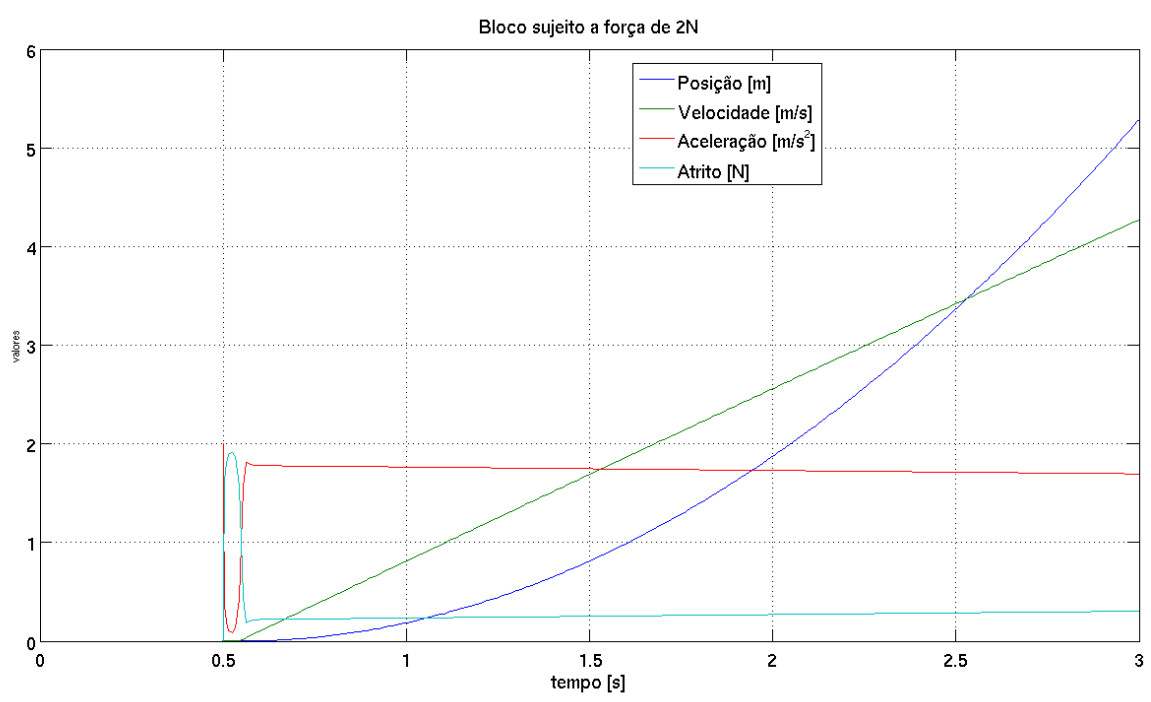

Figura 4.5: Ensaio do modelo de atrito, força de $2 \mathrm{~N}$ aplicada ao bloco

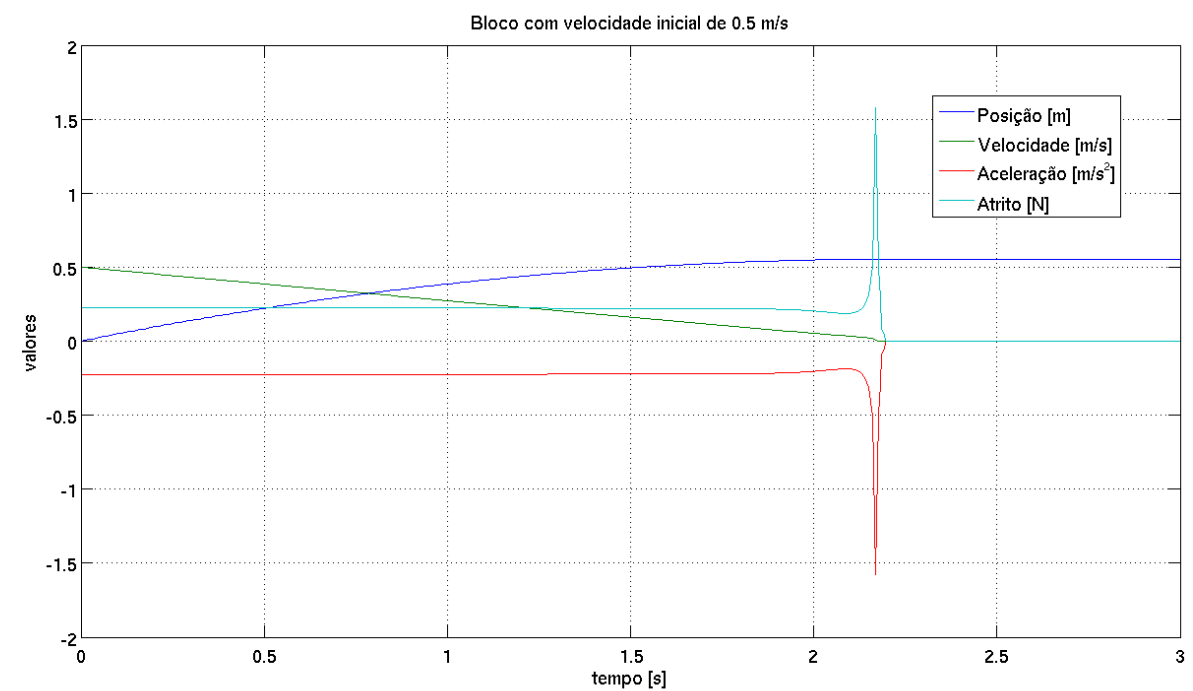

Figura 4.6: Ensaio do modelo de atrito, bloco com velocidade inicial.

\subsubsection{Motor como transdutor de torque}

Vamos considerar o motor como um transdutor linear de tensão em torque, ou seja, o torque será proporcional à tensão de armadura. Para tal, devemos desconsiderar a força contra eletromotriz induzida e encontrar a constante de proporcionalidade que para velocidades baixas pode ser aproximada por:

$$
K \tau_{, M O T}=\frac{K_{M O T}}{R a_{M O T}}
$$


desta forma:

$$
\tau_{M O T}=V a \times K \tau_{, M O T}
$$

\subsubsection{Modelo linearizado}

Para o projeto de controle, assumiremos inicialmente um modelo simplificado e linearizado do sistema. Se tomarmos as equações (4.27), linearizada em torno de $\bar{\phi}=0$ e (4.29), podemos então escrever em notação matricial, num modelo que desconsidera a posição $\theta$ e a velocidade $\dot{\theta}$ do disco:

$$
\begin{aligned}
& \dot{x}=\left(\begin{array}{cc}
0 & 1 \\
\mathbb{C}_{1} \mathbb{C}_{3} & 0
\end{array}\right) x+\left(\begin{array}{c}
0 \\
-\frac{\mathbb{C}_{1} K_{M O T}}{R a_{M O T}}
\end{array}\right) u \\
& y=\left(\begin{array}{ll}
1 & 0
\end{array}\right) x+(0) u
\end{aligned}
$$

Onde $x=\left(\begin{array}{c}\phi \\ \dot{\phi}\end{array}\right)$, a entrada é a tensão de armadura $u=V a$, e a saída $y=\phi$.

\subsubsection{Parâmetros numéricos do modelo}

O modelo escolhido deve ter seus parâmetros escolhidos de maneira a representar fielmente o comportamento do sistema construído.

Alguns parâmetros podem ser determinados facilmente pelo projeto, como por exemplo, os momentos de inercia. Para estes parâmetros, é de se esperar que os valores calculados sejam muito próximos dos reais.

Já parâmetros como os representativos do atrito, a menos de aproximações grosseiras, não são previamente conhecidos. Devem portanto ser determinados com o auxílio de ensaios.

A esta altura, temos condições de encontrar os valores numéricos das constantes do sistema mecânico e do motor, bem como, a partir de experimentos em bancada, determinar o comportamento aproximado do atrito. 
Dimensões e massas do sistema mecânico

A tabela a seguir apresenta as propriedades físicas e seus valores. 


\begin{tabular}{|c|c|c|}
\hline Simbolo & Valor & Descrição \\
\hline$g$ & $9.8\left[\frac{m}{s^{2}}\right]$ & Aceleração da gravidade. \\
\hline$\eta$ & $0,035[\mathrm{rad}]$ & $\begin{array}{l}\text { Inclinação do plano da barra em } \\
\text { relação ao plano horizontal }\end{array}$ \\
\hline$L_{B A R 1}$ & $0.2[\mathrm{~m}]$ & $\begin{array}{c}\text { Distância, no plano da barra, entre a } \\
\text { articulação desta e o motor. }\end{array}$ \\
\hline$L_{B A R 2}$ & $0.1[\mathrm{~m}]$ & $\begin{array}{c}\text { Distância, no plano da barra, entre a } \\
\text { articulação desta e o contra peso. }\end{array}$ \\
\hline$M_{B A R 1}$ & $0.141[\mathrm{Kg}]$ & $\begin{array}{c}\text { Massa da barra de alumínio, relativa } \\
\text { apenas ao braço do motor. }\end{array}$ \\
\hline$M_{B A R 2}$ & $0.0799[\mathrm{Kg}]$ & $\begin{array}{c}\text { Massa da barra de alumínio, relativa } \\
\text { apenas ao braço do contrapeso. }\end{array}$ \\
\hline$M_{M O T}$ & $0.31[\mathrm{Kg}]$ & Massa do motor. \\
\hline$M_{C I L}$ & $0.546[\mathrm{Kg}]$ & Massa do contra peso. \\
\hline$M_{D I S}$ & $0.458[\mathrm{Kg}]$ & Massa do disco de alumínio. \\
\hline$J_{B A R 1}$ & $0.00229\left[K g \times m^{2}\right]$ & $\begin{array}{l}\text { Momento de inércia do primeiro } \\
\text { braço da barra em relação à sua } \\
\text { articulação. }\end{array}$ \\
\hline$J_{B A R 2}$ & $0.000422\left[K g \times m^{2}\right]$ & $\begin{array}{l}\text { Momento de inércia do segundo } \\
\text { braço da barra em relação à sua } \\
\text { articulação. }\end{array}$ \\
\hline$J_{M O T}$ & $0.0125\left[K g \times m^{2}\right]$ & $\begin{array}{l}\text { Momento de inércia do motor em } \\
\text { relação à articulação da barra. }\end{array}$ \\
\hline$J_{C I L}$ & $0.00544\left[K g \times m^{2}\right]$ & $\begin{array}{l}\text { Momento de inércia do contra peso } \\
\text { em relação à articulação da barra. }\end{array}$ \\
\hline$J_{D I S}$ & $0.00289\left[K g \times m^{2}\right]$ & $\begin{array}{l}\text { Momento de inércia do disco (e } \\
\text { flange) em relação ao seu centro de } \\
\text { rotação própria (eixo do motor) }\end{array}$ \\
\hline$V E L_{M O T}$ & $270.177\left[\frac{\mathrm{rad}}{s}\right]$ & $\begin{array}{c}\text { Rotação nominal do motor quando } \\
\text { submetido a tensão nominal de } 12 \\
\text { volts. }\end{array}$ \\
\hline$K_{M O T}$ & $0.0265\left[\frac{N}{A}\right]$ & $\begin{array}{l}\text { Constante de torque do motor, } \\
\text { conforme definido pelo fabricante. }\end{array}$ \\
\hline$R a_{M O T}$ & $1.94[\Omega]$ & $\begin{array}{c}\text { Resistência elétrica da armadura do } \\
\text { motor. }\end{array}$ \\
\hline
\end{tabular}


Podemos então determinar os valores das constantes $\mathbb{C}_{1}, \mathbb{C}_{2}$ e $\mathbb{C}_{3}$ :

$$
\begin{aligned}
& \mathbb{C}_{1}=\frac{1}{0.00544+0.00229+0.000422+0.0125+(0.2)^{2} 0.458}=\frac{1}{0.038972}=25.66 \\
& \mathbb{C}_{2}=\frac{1}{0.00289}=346.02 \\
& \mathbb{C}_{3}=1.069229 \cdot \sin (\eta)=0.03743
\end{aligned}
$$

\section{Atrito no motor}

Podemos ainda, determinar de forma aproximada, os coeficientes de atrito do motor, pois sabemos sua rotação nominal, a constante de torque e a resistência de armadura. Quando em regime permanente, o torque gerado pelo motor será completamente consumido pelo atrito do rotor em velocidade nominal $(\mathrm{rad} / \mathrm{s})$.

A tensão contra eletromotriz será:

$$
V_{\text {cem }}=270.177 \cdot K_{M O T}=270.177 \cdot 0.0265=7.1597
$$

A corrente resultante sobre a armadura é:

$$
\text { Ia } a_{M O T}=\frac{12-7.1597}{1.94}=2.495
$$

E o torque de atrito total resulta:

$$
\tau_{\text {tot }, a t, M O T}=0.0661
$$

Em ensaios, verificou-se que a mínima tensão aplicada à armadura do motor que é capaz de tirar o rotor do repouso está em torno de 0.8 volts. Então, podemos estimar o torque de atrito estático que nesta condição vale:

$$
\tau_{e, a t, M O T}=\frac{0.8}{1.94} \cdot 0.0265=0.0109
$$

Supondo o torque de atrito cinético em torno de 20 por cento do atrito estático, temos:

$\tau_{c, a t, M O T}=0.0022$

E por fim, a constante de atrito viscoso no motor será dada por:

$$
\tau_{v, a t, M O T}=\frac{0.0661-0.0027}{270.177}=0.00023663
$$

Tomando uma velocidade limiar para o atrito estático em torno de $10^{-4}$ da velocidade máxima do rotor, ou seja, $0.027 \mathrm{rad} / \mathrm{s}$, e uma velocidade de $0.1 \mathrm{rad} / \mathrm{s}$ para o limiar de atrito cinético, as constantes do modelo de atrito foram estimadas em:

$$
\gamma_{\text {MOT }}=\left(\begin{array}{llllll}
0.2809 & 54.58 & 49.9825 & 0.0027 & 60.2917 & 0.0002346
\end{array}\right)
$$


E para estas constantes, o gráfico de atrito em função da velocidade do rotor é exibido nas figuras 4.7 e 4.8 :

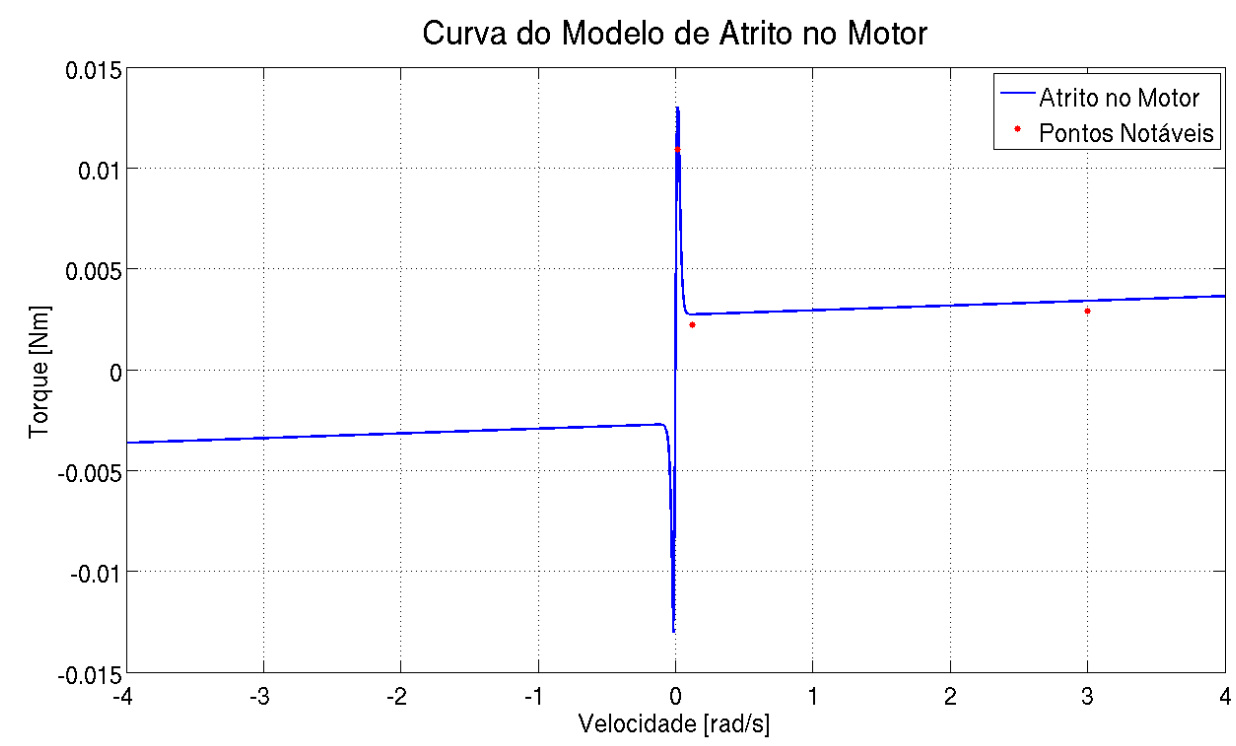

Figura 4.7: Gráfico de comportamento do modelo de atrito.

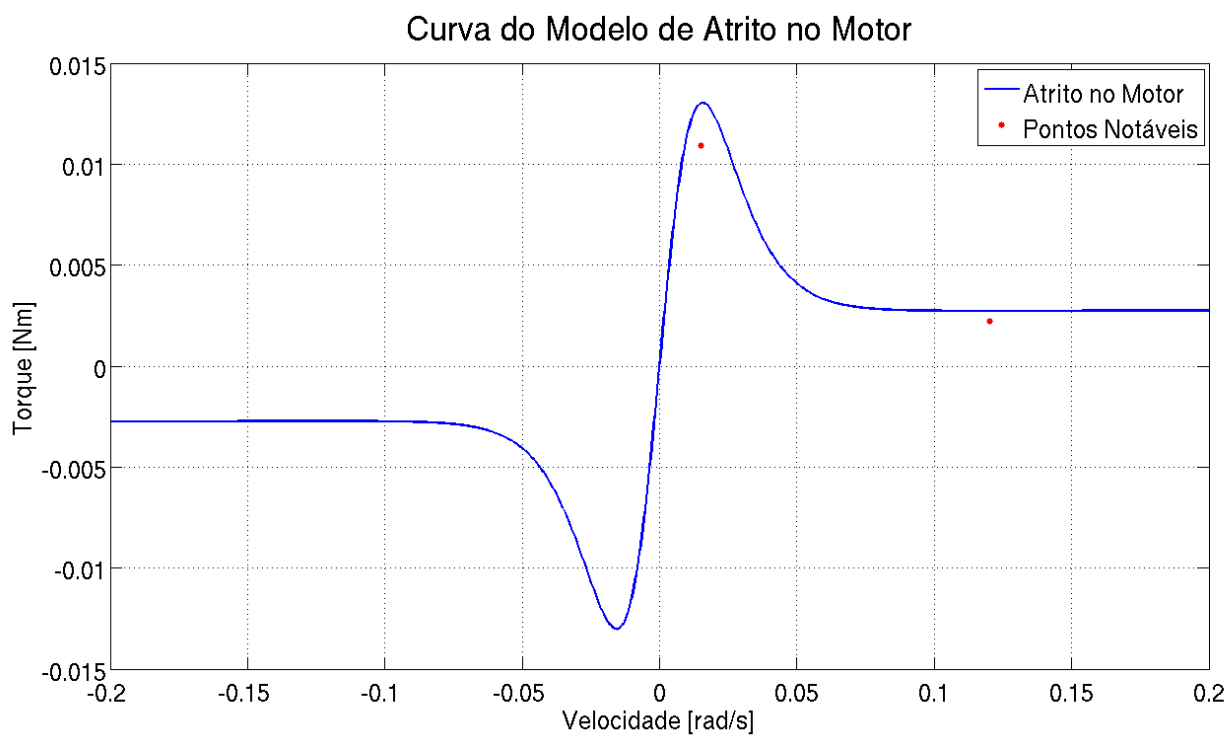

Figura 4.8: Gráfico de comportamento do modelo de atrito.

\section{Atrito na barra}

Em ensaios realizados em bancada, com o controle desligado, observou-se que existe uma região em torno do ponto de equilíbrio instável, na qual não existe movimento da barra. Nesta região, o torque gerado pela atuação da força da gravidade no centro de massa do mecanismo 
da barra não é suficiente para vencer o torque de atrito estático na articulação da barra. Esta região foi determinada como sendo aproximadamente 6 por cento de uma revolução completa da barra, $-3 \%$ a $+3 \%$, em torno do ponto de equilíbrio instável. No limiar de movimentação, o torque gerado pela gravidade iguala-se ao torque de atrito estático, então:

$$
\tau_{e, a t, B A R}=\left\|\mathbb{C}_{3} \sin (0.03 * 2 \pi)\right\|=0.0093
$$

Para estimar-se o atrito dinâmico, vamos utilizar os resultados de ensaio, devidamente filtrados com o auxílio de um filtro Savitzky-Golay [12], onde utilizaremos uma janela de 501 amostras, 250 antes do ponto em análise e 250 depois. O filtro Savitzky-Golay é uma técnica de filtragem off-line de janela móvel, onde o valor (e suas derivadas) em cada amostra é obtido pela aproximação de um polinômio de grau fixo que garanta a minimização dos quadrados dos desvios dentro de cada janela. Esta técnica permite a obtenção de um sinal razoavelmente livre de ruídos e boas estimativas para suas derivadas, mas por se utilizar de amostras futuras, não pode ser empregada em sistemas de tempo real.

Os resultados de ensaio, filtrados e o torque teórico são mostrados na figura 4.9.

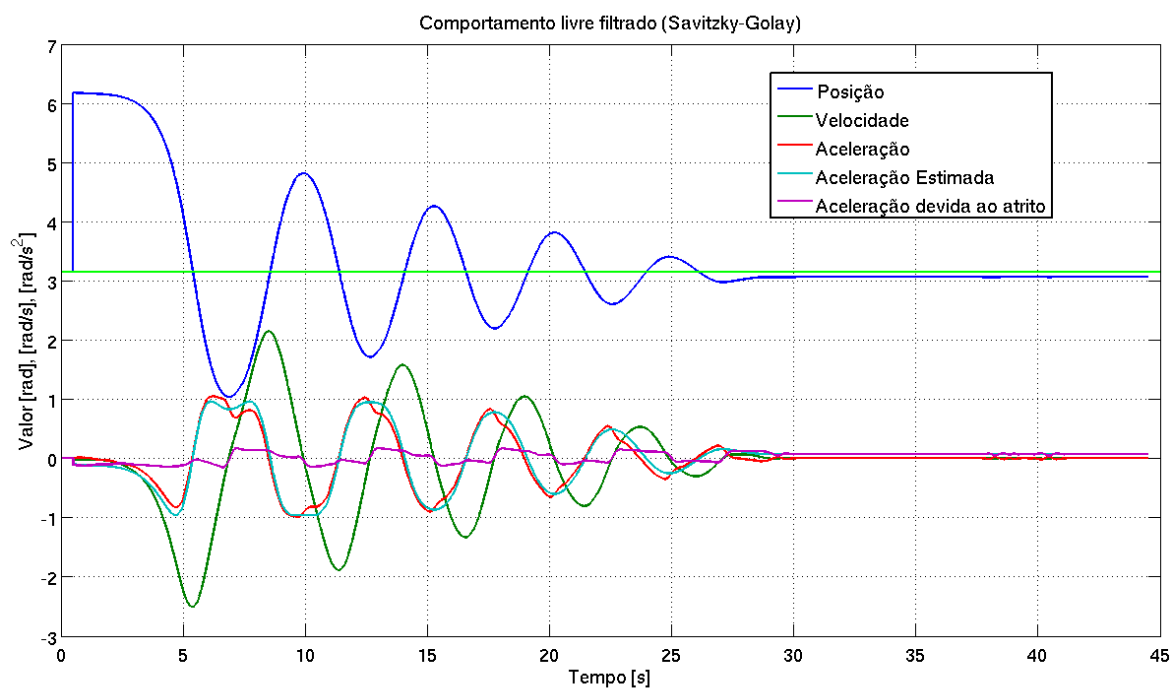

Figura 4.9: Resultado de ensaio, após filtragem.

A curva em vermelho representa a aceleração realmente obtida no sistema, enquanto que a curva em ciano representa a aceleração teórica. A diferença entre as duas é uma estimativa da aceleração devida ao torque de atrito e é representada em roxo.

A seguir plotamos as diversas acelerações de atrito em função da velocidade. Nosso intuito é aproximar, via método dos mínimos quadrados, duas retas que modelem o atrito cinético e viscoso como função da velocidade. Para esta aproximação, eliminamos os pontos para os 
quais o módulo da velocidade é muito pequeno.

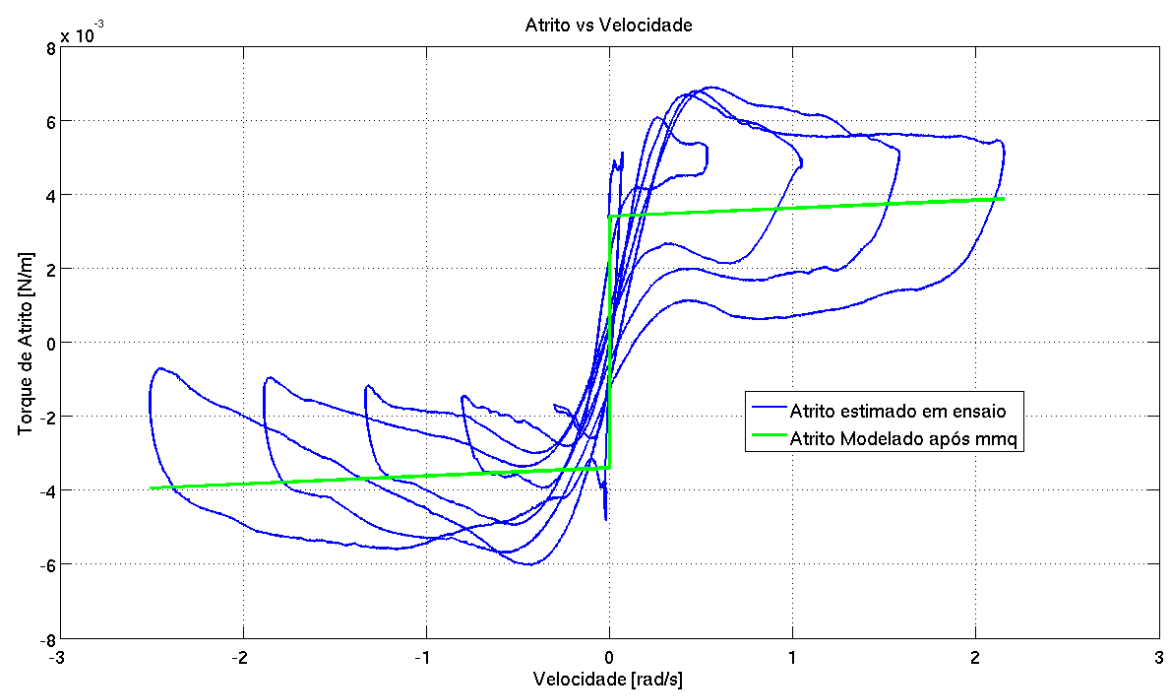

Figura 4.10: Atrito cinético + viscoso.

Por fim, ilustramos o modelo de atrito obtido para as constantes estimadas:

$$
\gamma_{B A R}=\left(\begin{array}{llllll}
0.03686 & 5388 & 3250 & 0.003446 & 1742.7 & 0.0002199
\end{array}\right)
$$

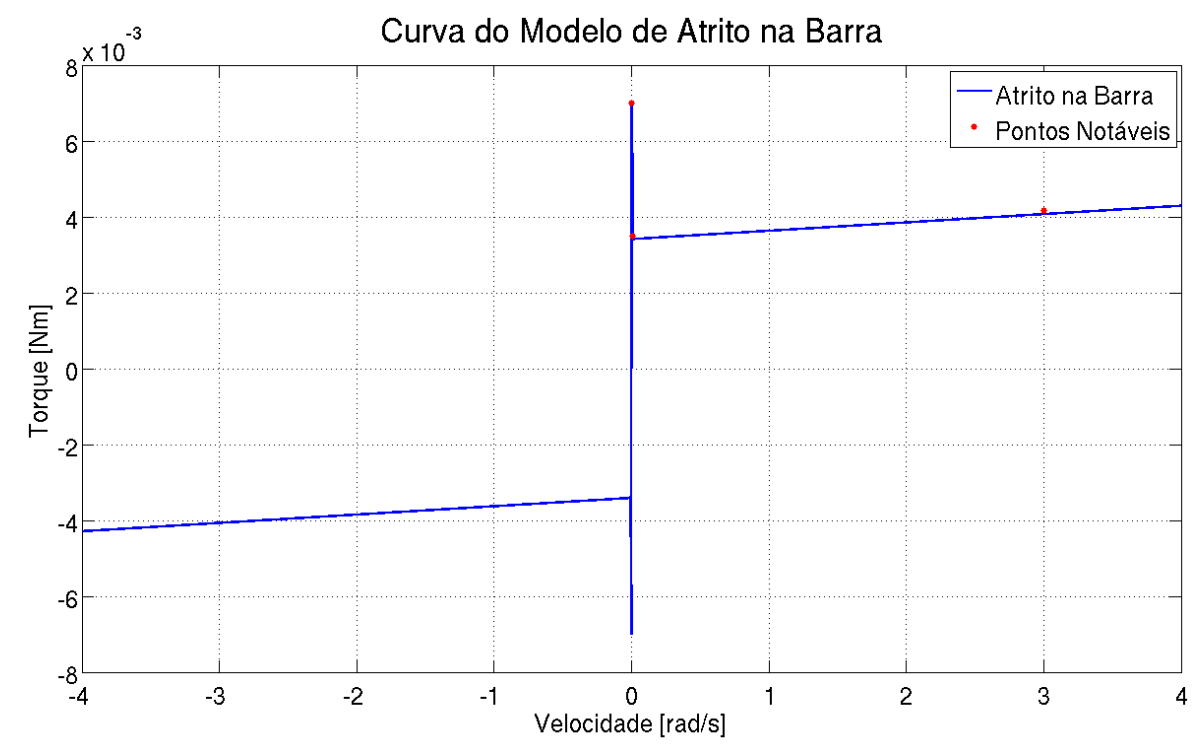

Figura 4.11: Atrito cinético + viscoso. 


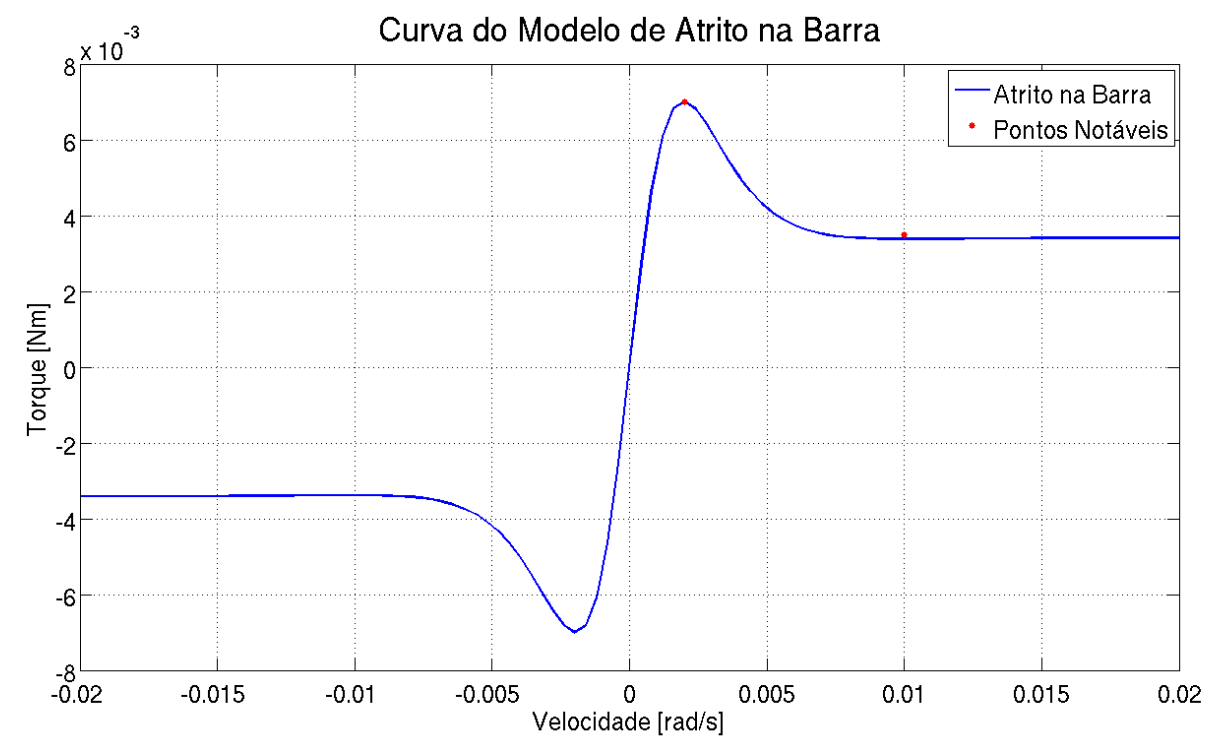

Figura 4.12: Detalhe do Atrito cinético + viscoso.

Finalizando este capítulo, apresentamos as equações do modelo construído e mostramos os resultados de ensaio.

O modelo do sistema mecânico será descrito pelas seguintes equações:

$$
\begin{gathered}
\ddot{\phi}=-25.66 \tau_{M O T}-25.66 \tau_{a t, D I S}+25.66 \tau_{a t, B A R}+0.9605 \sin (\phi) \\
\ddot{\theta}=371.68 \tau_{M O T}+371.68 \tau_{a t, D I S}-25.66 \tau_{a t, B A R}+0.9605 \sin (\phi) \\
\tau_{M O T}=0.0137 v_{a}-0.00036198 \dot{\theta}
\end{gathered}
$$




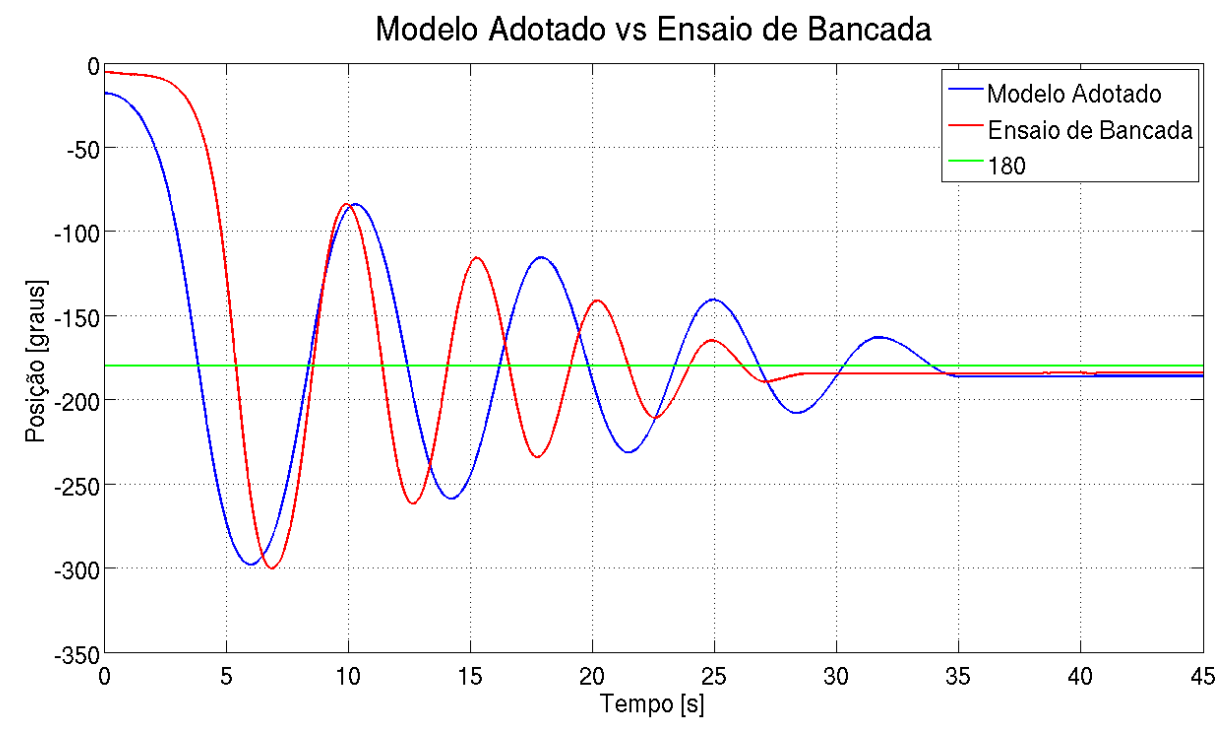

Figura 4.13: Modelo e Ensaio

\section{Modelo simplificado e linearizado}

O modelo simplificado e linearizado, descrito matricialmente por (4.30), fica:

$$
\begin{aligned}
\dot{x} & =\left(\begin{array}{cc}
0 & 1 \\
0.9605 & 0
\end{array}\right) x+\left(\begin{array}{c}
0 \\
-0.3505
\end{array}\right) u \\
y & =\left(\begin{array}{ll}
1 & 0
\end{array}\right) x
\end{aligned}
$$




\section{Controle do Pêndulo por transmissão de torque}

Neste capítulo vamos projetar uma lei de controle simples e observar o funcionamento com o estimador proposto em comparação a um observador tradicional. 


\subsection{Realimentação linear de estados}

Para projetar uma lei de controle linear, precisamos do modelo linearizado do sistema, equação (4.34).

As matrizes de controlabilidade e observabilidade resultam:

$$
\begin{aligned}
& \mathbb{C}=\left(\begin{array}{cc}
0 & -0.3505 \\
-0.3505 & 0
\end{array}\right) \text {, com posto pleno. O sistema é completamente controlável. } \\
& \mathbb{O}=\left(\begin{array}{ll}
1 & 0 \\
0 & 1
\end{array}\right) \text {, com posto pleno. O sistema é observável. }
\end{aligned}
$$

Uma lei de controle capaz de alocar os pólos do sistema linearizado em $-2.5 \pm 2.5 i$, obtida via comando place do software MATLAB é:

$$
G=\left(\begin{array}{ll}
-38.4 & -14.2
\end{array}\right)
$$

O comportamento simulado desta realimentação de estados, em simulação conjunta com o modelo completo sujeito a limitação de tensão máxima de armadura de \pm 10 volts é mostrado na figura 5.1:

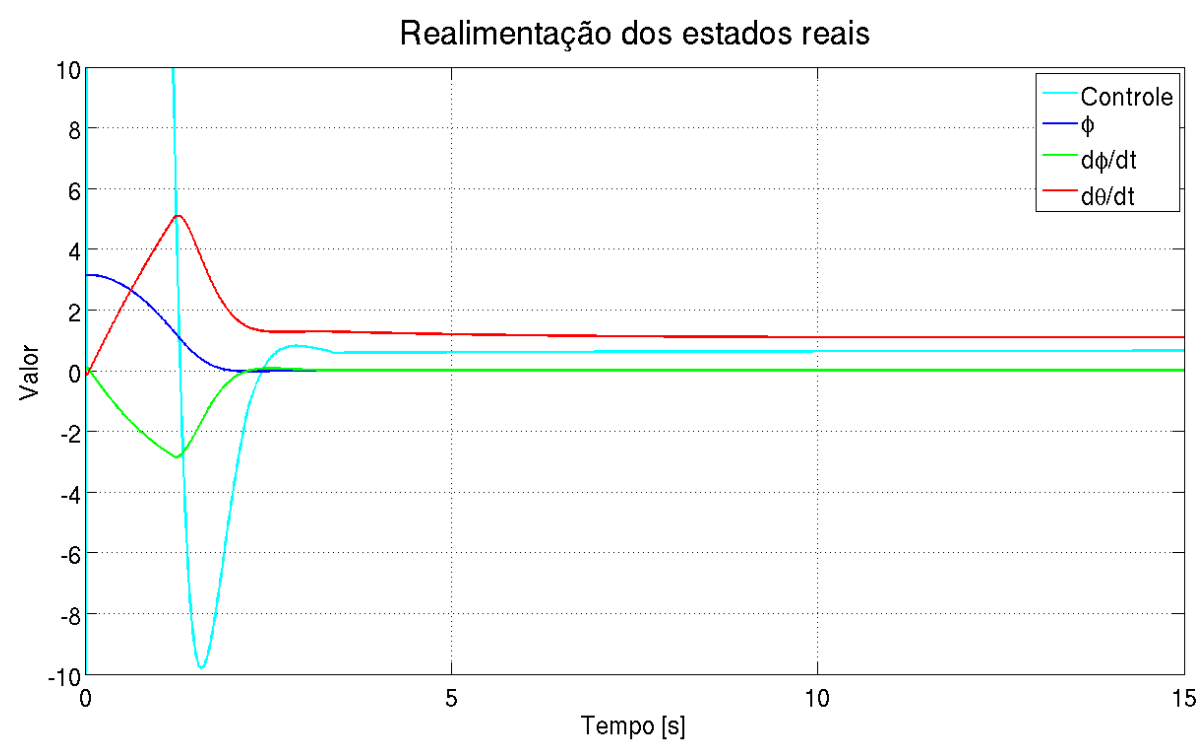

Figura 5.1: Simulação de Controle Linear 


\subsection{Observador assintótico para o sistema linearizado}

Sabemos que o subsistema considerado é observável, então podemos alocar livremente os pólos de um observador assintótico. A matriz de ganhos que aloca os pólos do observador em $-50 \pm 50 i$, também obtida via comando place do software MATLAB é:

$$
K=\left(\begin{array}{c}
100 \\
5001
\end{array}\right)
$$

O comportamento do sistema foi simulado nas mesmas condições da seção 5.1, na figura 5.2 ilustramos o comportamento obtido sem ruído. Na figura 5.3, apresentamos os resultados obtidos na presença de ruído aditivo de variância 0.001 :

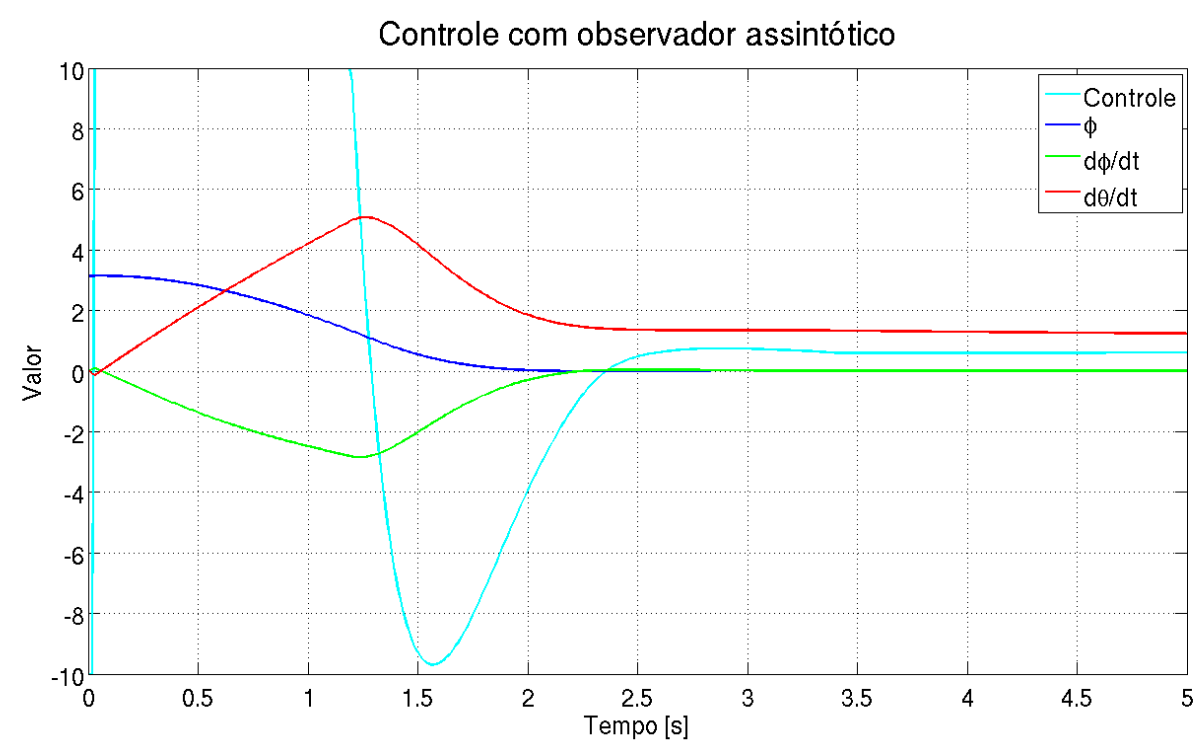

Figura 5.2: Simulação de Controle Linear por observadores assintóticos de estado. 


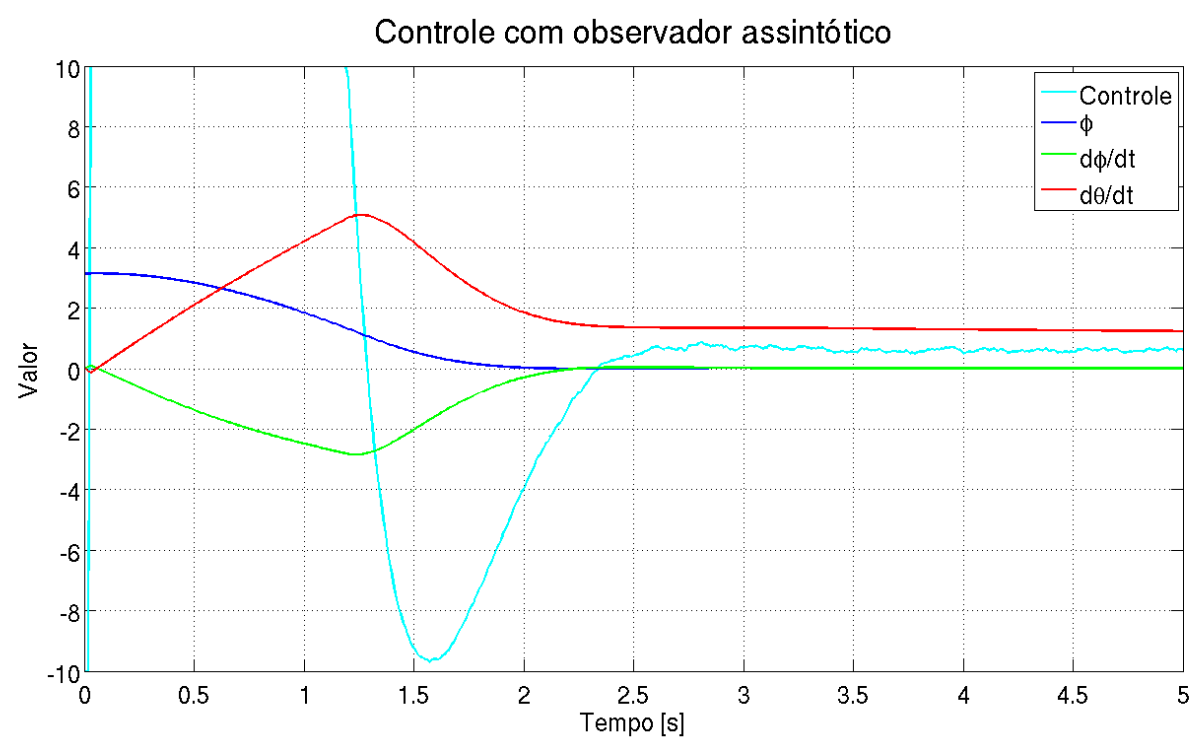

Figura 5.3: Simulação de Controle Linear por observadores assintóticos de estado em presença de ruído aditivo.

\subsection{Controle com estimador algébrico}

Utilizando dois estimadores de derivadas de ordem 7 intercalados e reinicializados alternadamente a cada 2 segundos (vide ilustração 2.1), construímos um observador não assintótico. Os estados observados são a saída medida pelo encoder, e sua primeira derivada estimada. Nas figura 5.4 exibimos o comportamento obtido via simulação e repetimos o resultado para uma saída corrompida por ruído aditivo de variância 0.001 na figura 5.5.

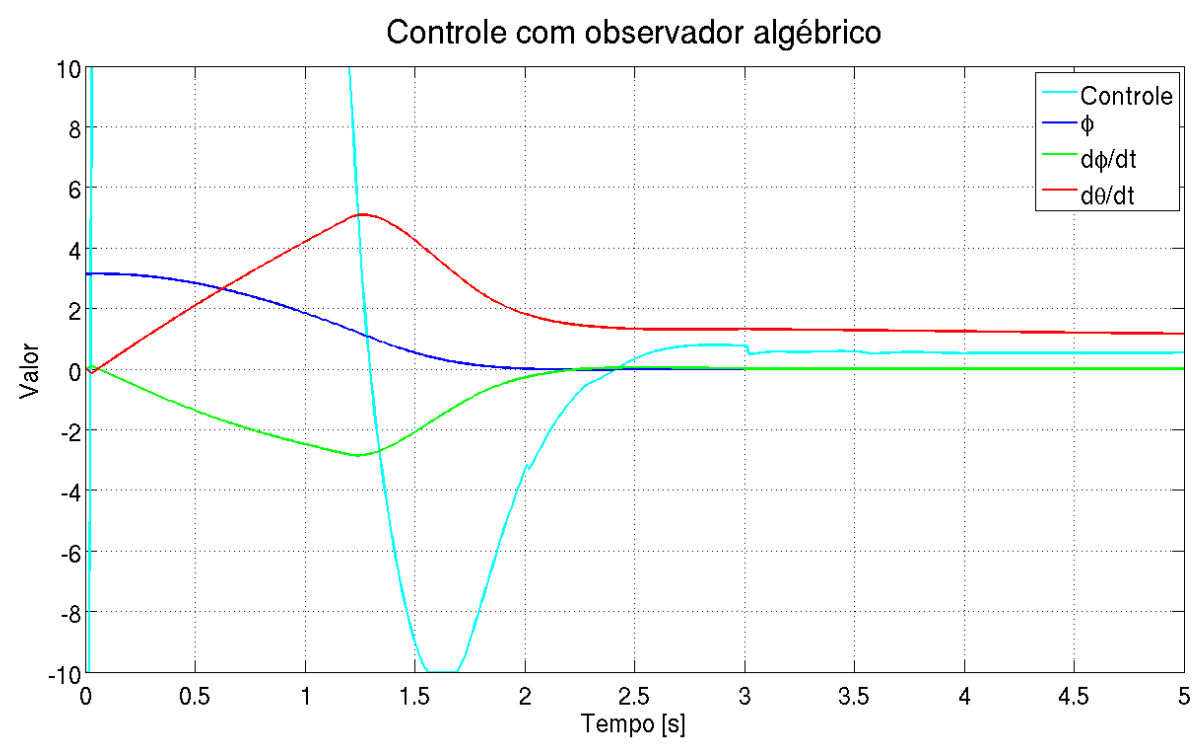

Figura 5.4: Simulação de Controle Linear por observador algébrico 


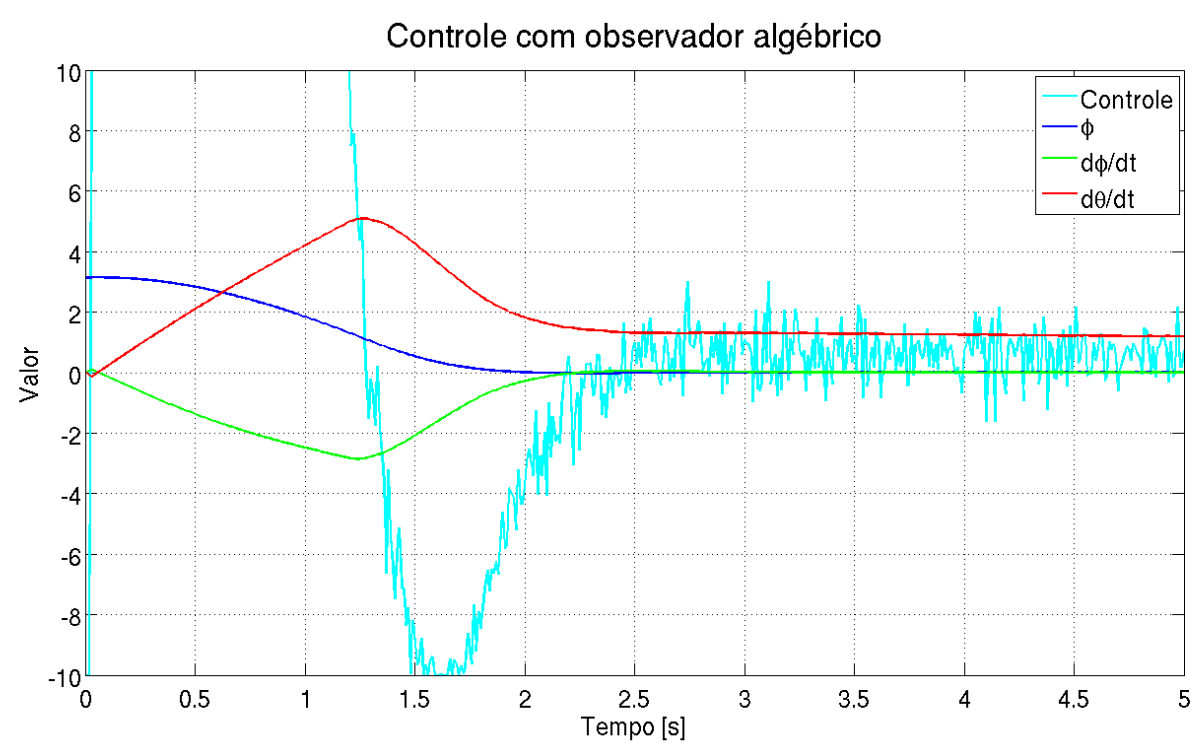

Figura 5.5: Simulação de Controle Linear por observador algébrico na presença de ruído

\subsection{Comparações entre os observadores}

Representamos nas figuras seguintes, algumas comparações entre os dois observadores construídos:

\subsubsection{Posicionamento do braço do pêndulo.}

As figuras 5.6 e 5.8 ilustram o comportamento obtido no posicionamento do braço do pêndulo em simulação com e sem ruído. 


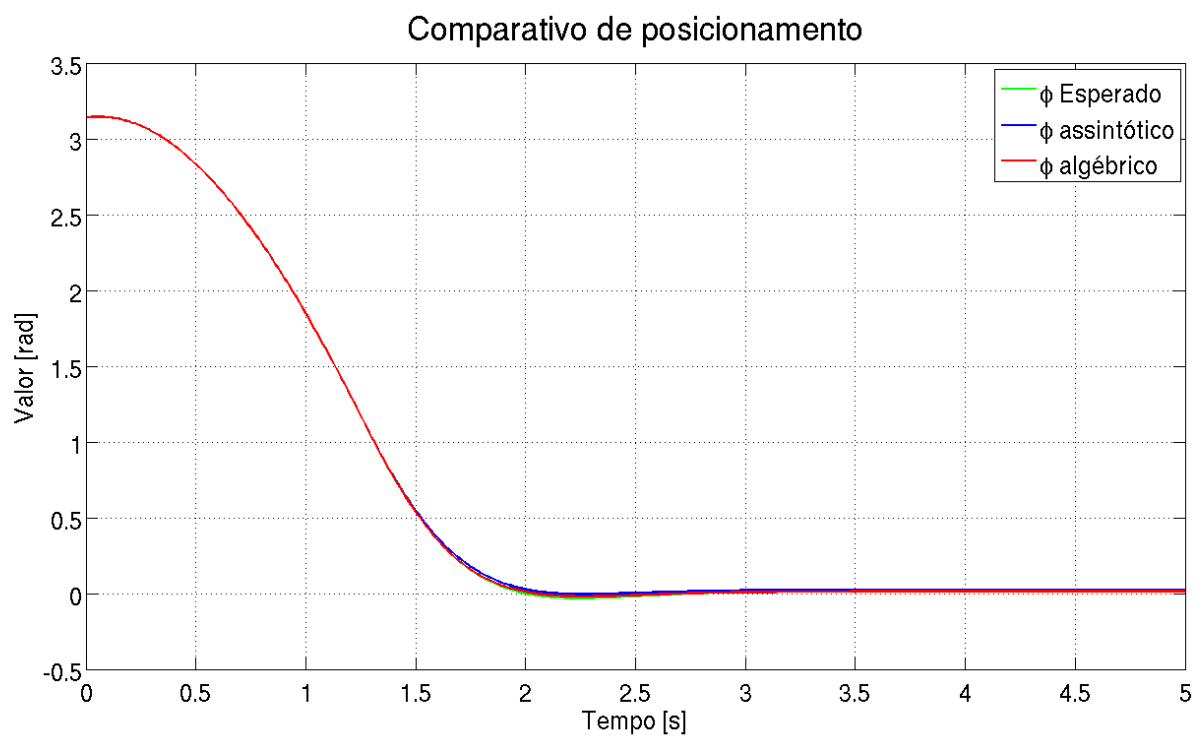

Figura 5.6: Comparativo de posicionamento.

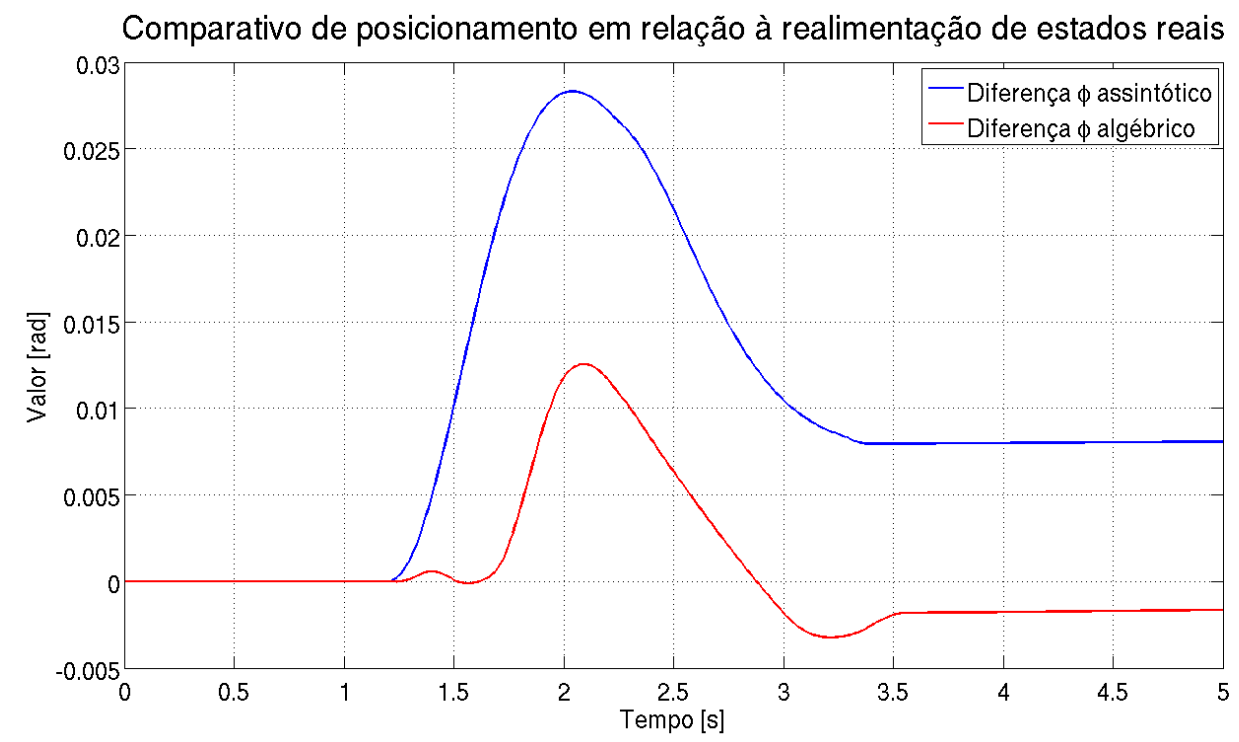

Figura 5.7: Diferença de posicionamento do braço em relação à realimentação de estados reais. 


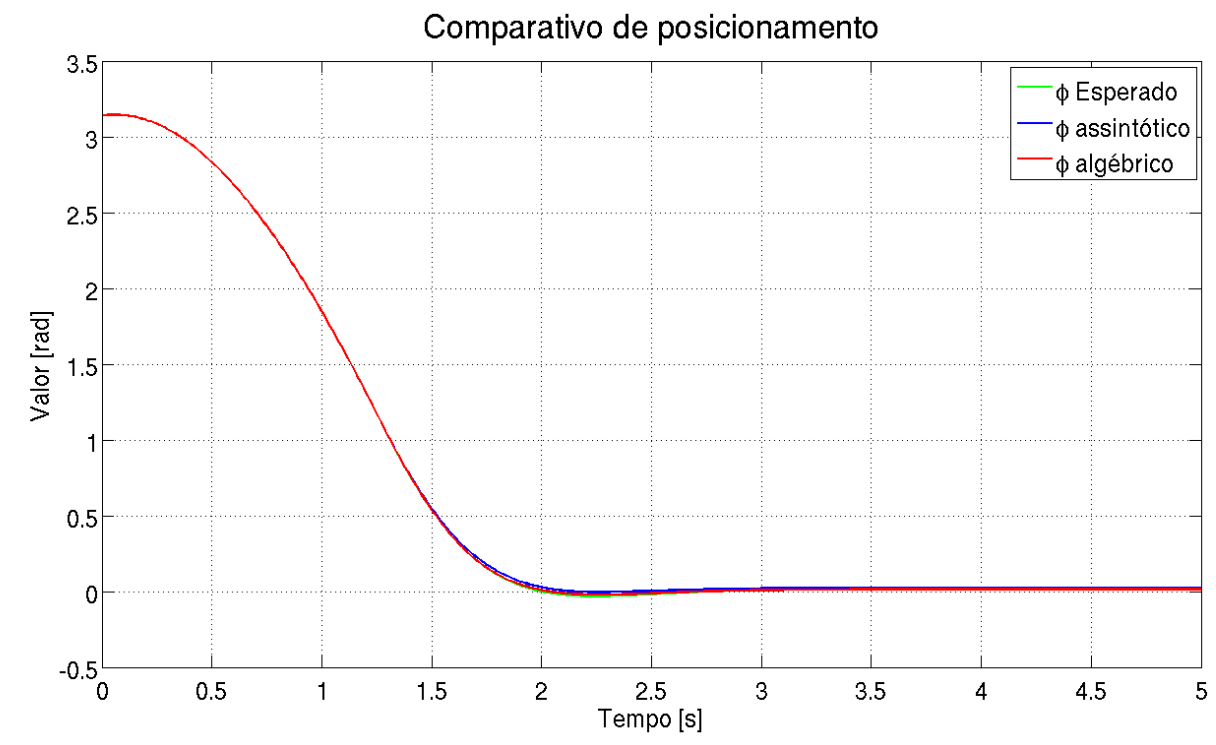

Figura 5.8: Comparativo de posicionamento em ambiente ruidoso.

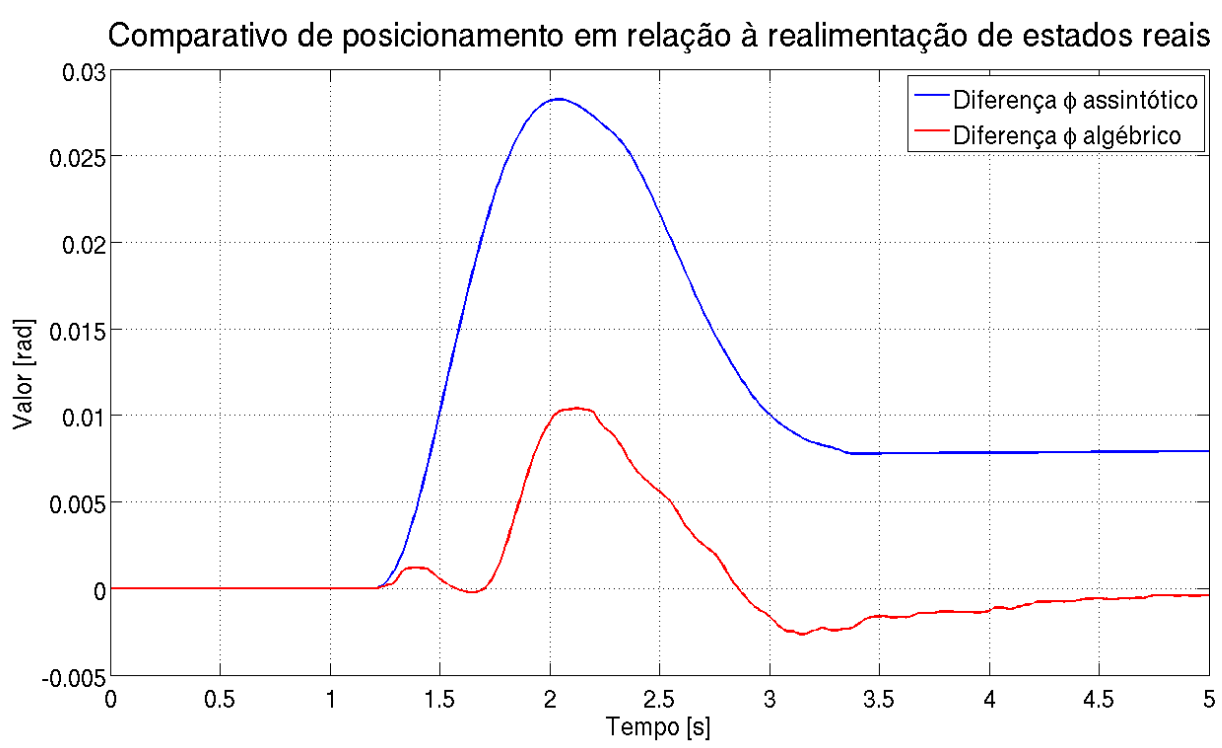

Figura 5.9: Diferença de posicionamento em relação à realimentação de estados reais, ambiente ruidoso.

Verifica-se que na ausência de ruídos, o estimador algébrico produz um comportamento mais próximo da realimentação pura de estados

\subsubsection{Estimador de estados $\phi$}

Apresentamos o comportamento dos estimadores de estado para a variável $\phi$ (algébrico e observador assintótico) e seu erro em relação ao estado real. 


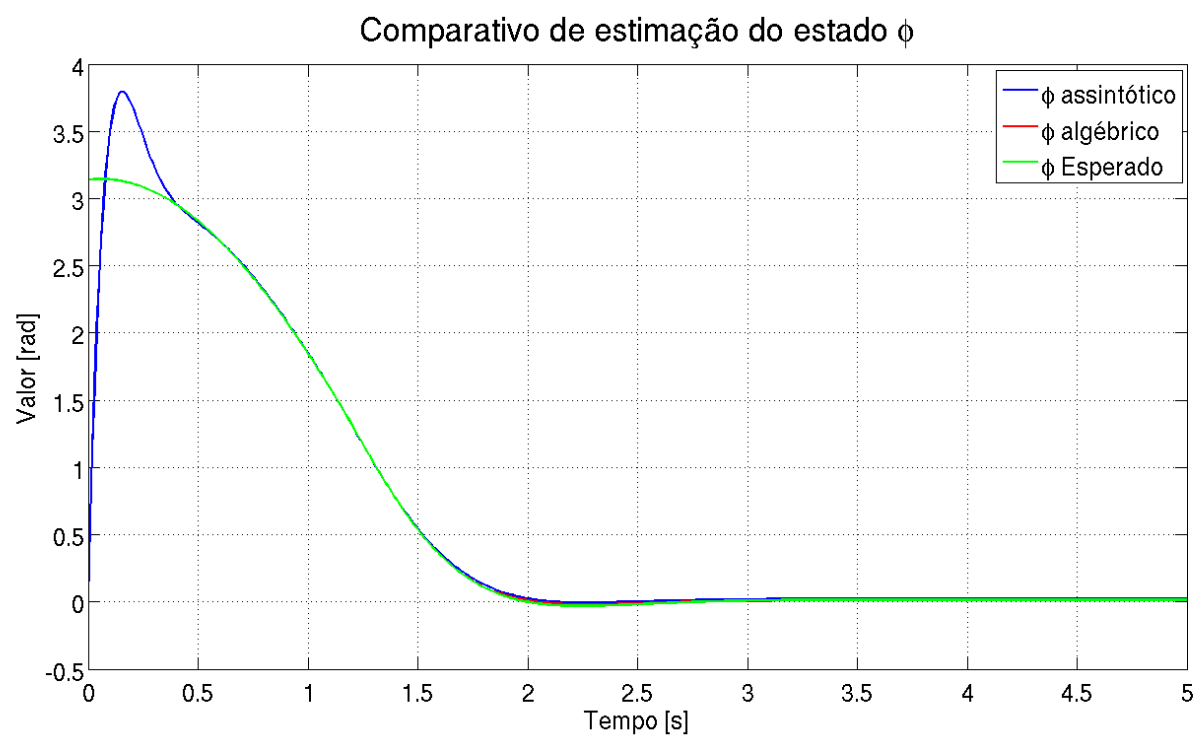

Figura 5.10: Valores Estimados de $\phi$

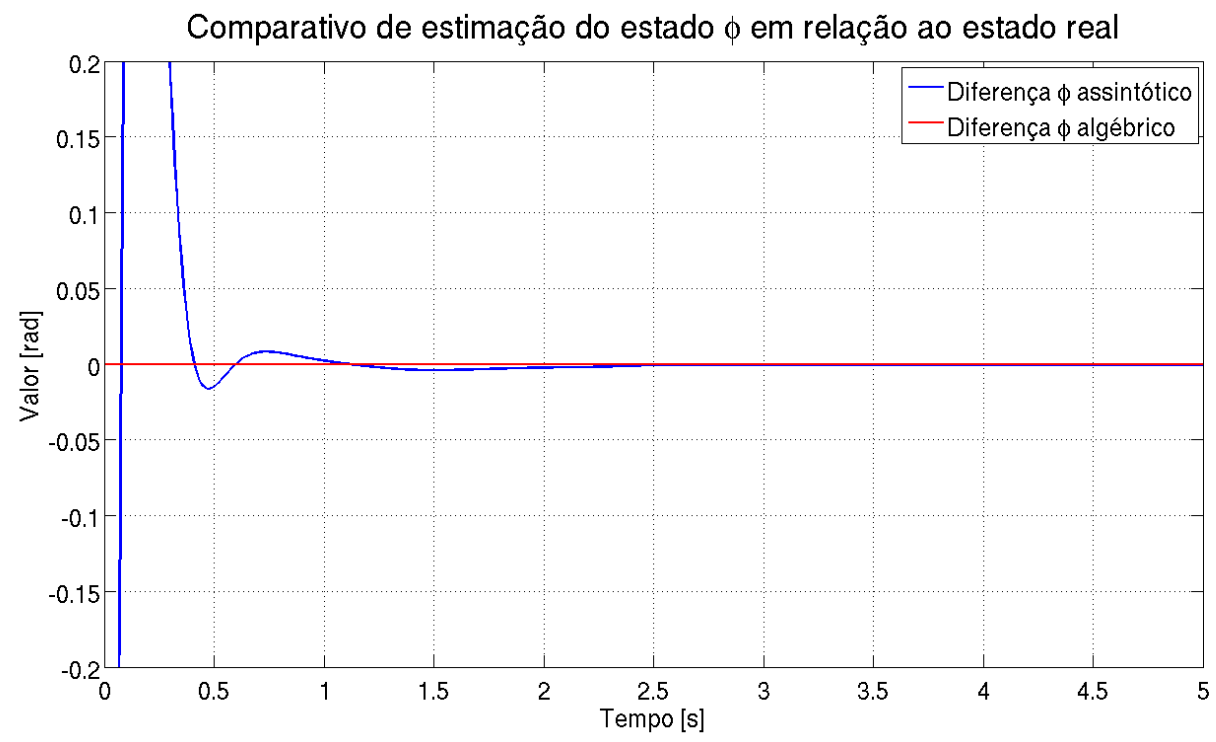

Figura 5.11: Diferença entre os valores estimados e o real valor de $\phi$.

Nas figuras 5.12 e 5.13, repetimos os gráficos anteriores agora com a saída do sistema corrompida por ruído aditivo. 


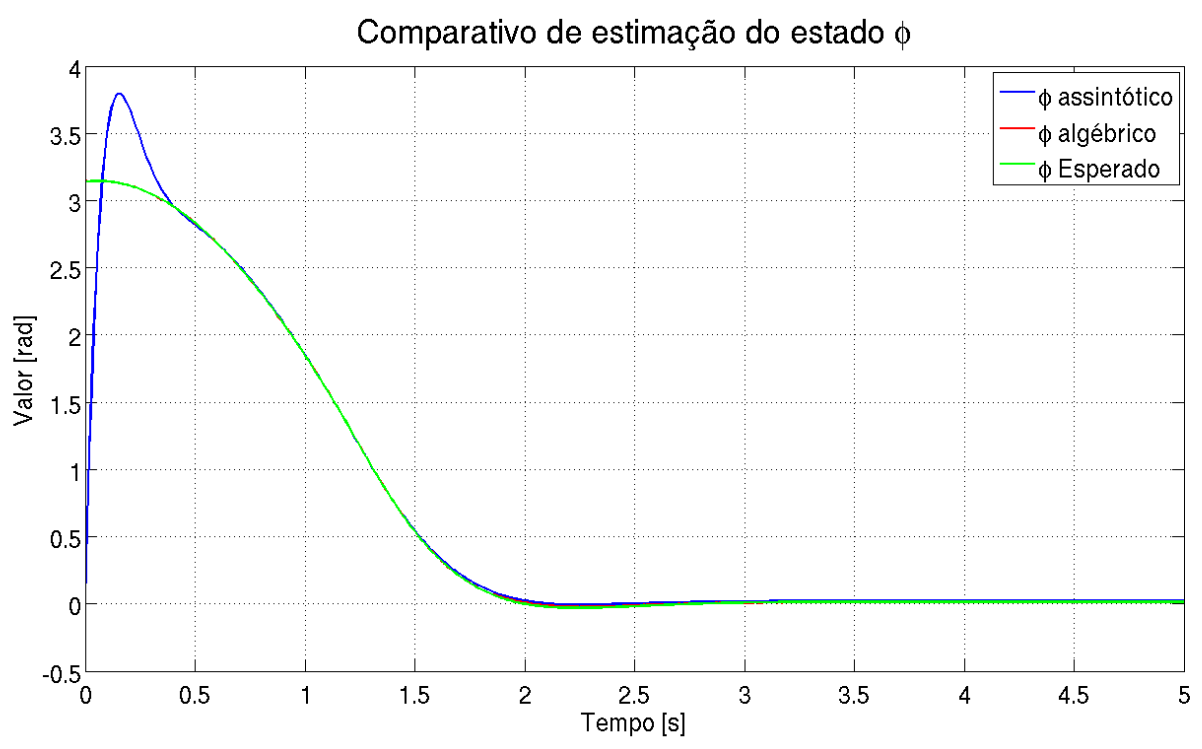

Figura 5.12: Valores Estimados de $\phi$

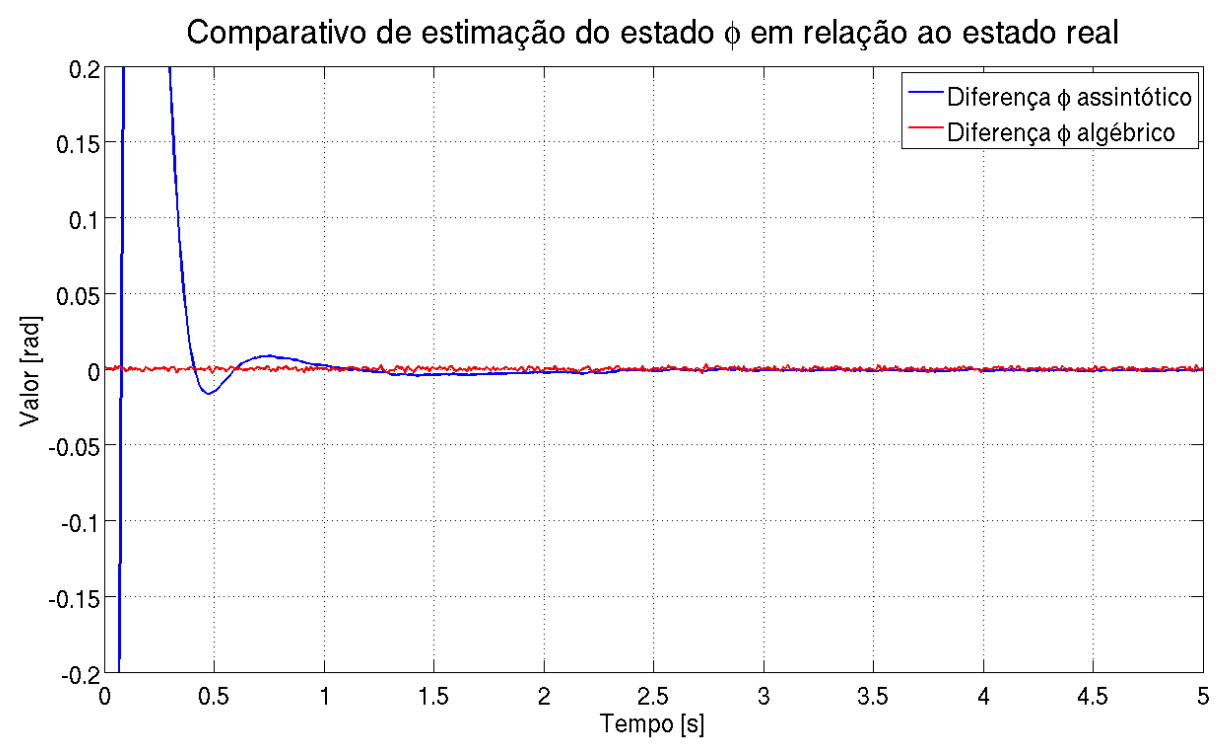

Figura 5.13: Diferença entre os valores estimados e o real valor de $\phi$, ambiente ruidoso.

\subsubsection{Estimador de estados $\dot{\phi}$}

Apresentamos o comportamento dos estimadores de estado para a variável $\dot{\phi}$ (algébrico e observador assintótico) e seu erro em relação ao estado real. 


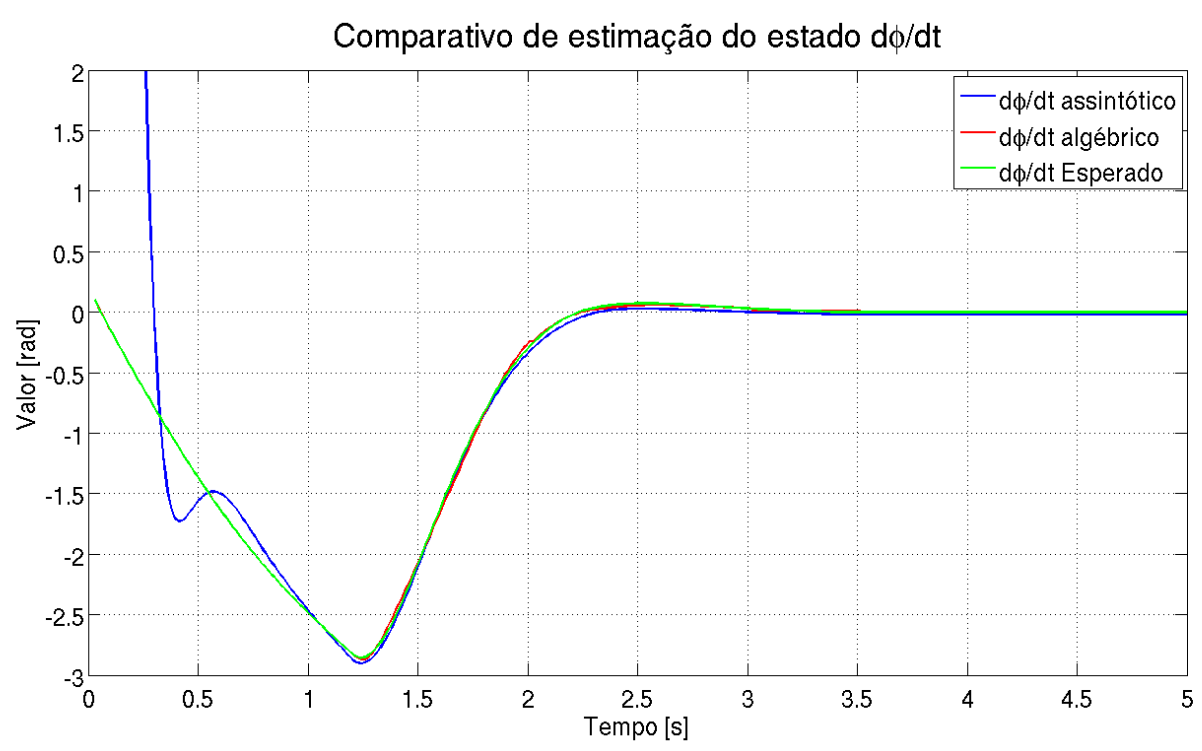

Figura 5.14: Valores Estimados de $\dot{\phi}$

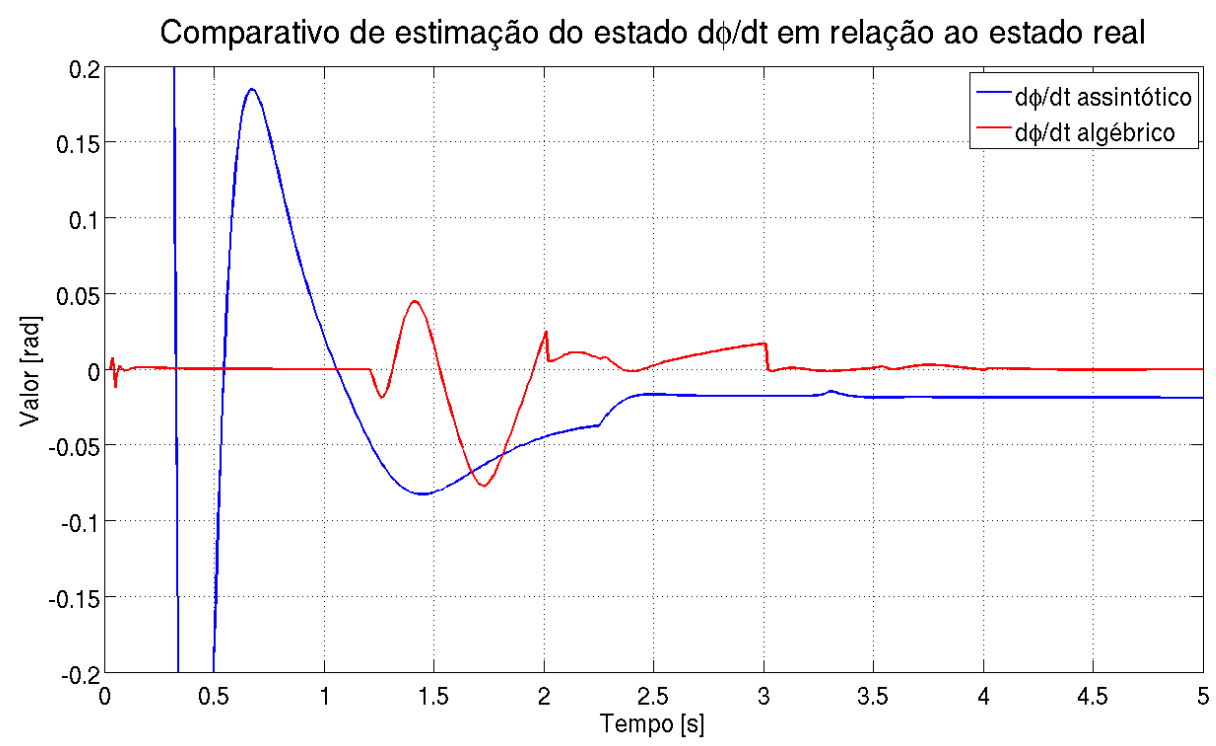

Figura 5.15: Diferença entre os valores estimados e o valor real de $\dot{\phi}$.

Nas figuras 5.16 e 5.17, repetimos os gráficos anteriores agora com a saída do sistema corrompida por ruído aditivo. 


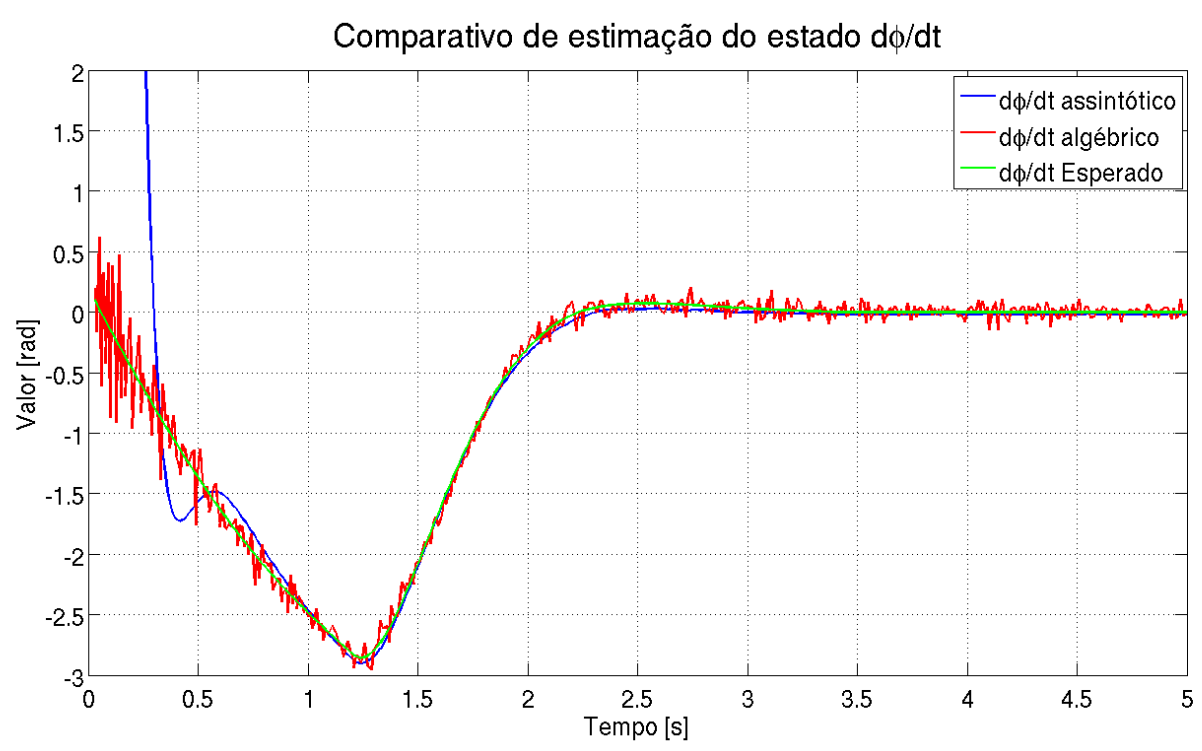

Figura 5.16: Valores Estimados de $\dot{\phi}$, ambiente ruidoso.

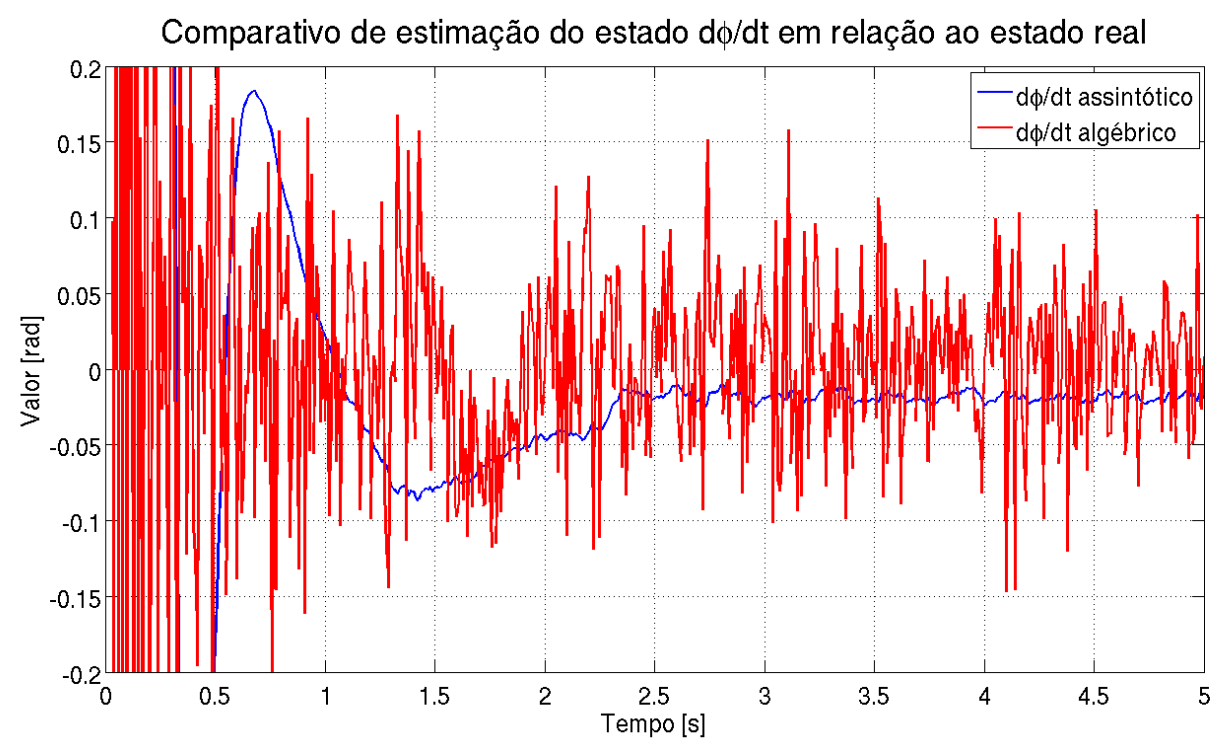

Figura 5.17: Diferença entre os valores estimados e o valor real de $\dot{\phi}$, ambiente ruidoso.

Na figura 5.17 podemos observar que o erro de estimação, apesar de diminuto é maior no estimador algébrico. Isto ocorre pois o estimador é rápido e não se utilizou nenhum tipo de filtragem adicional. Já o estimador assintótico, por ser mais lento, apresentou um resultado mais preciso e livre de ruídos após o tempo de convergência. 


\subsection{Ensaio de Bancada com o pêndulo por transferência de torque}

\subsubsection{Comparativo do resultado de controle}

A lei de controle e o estimador proposto nos ensaios anteriores foi implementada no controle do sistema construído. O primeiro gráfico deste ensaio, figura 5.18, é exibido a seguir e demonstra o posicionamento do braço do pêndulo (variável $\phi$ ) em ambos os tipos de controle.

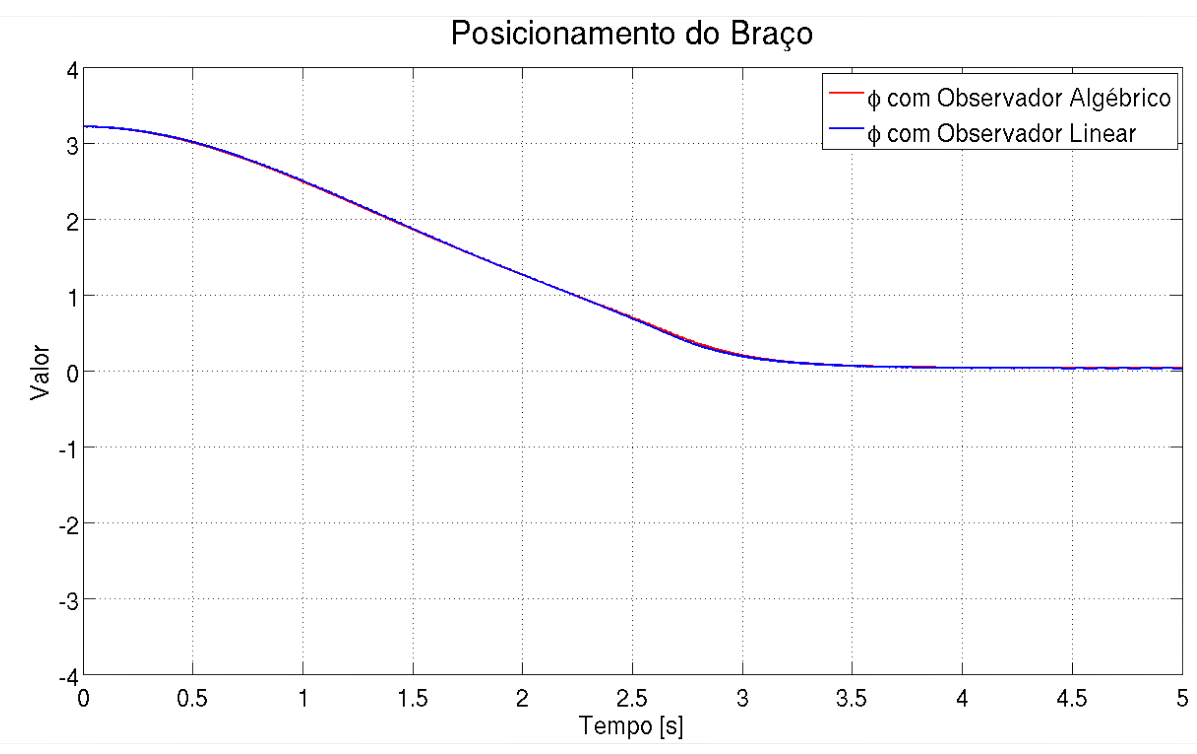

Figura 5.18: Posicionamento do braço no ensaio em bancada.

Não se percebe diferença significativa entre os controles, ambos atenderam muito bem às expectativas.

\subsubsection{Comparativo entre os observadores}

Nas figuras 5.19, 5.20, 5.21 e 5.22, exibimos o comportamento dos observadores de estados. Foram realizados diversos ensaios utilizando os dois tipos de observadores e os resultados foram muito repetitivos. Inclusive, como histórico de posicionamento de ambas as construções é praticamente idêntico (ver figura 5.18), assumimos a média entre os dois tipos de controle como referência nas comparações a seguir. 


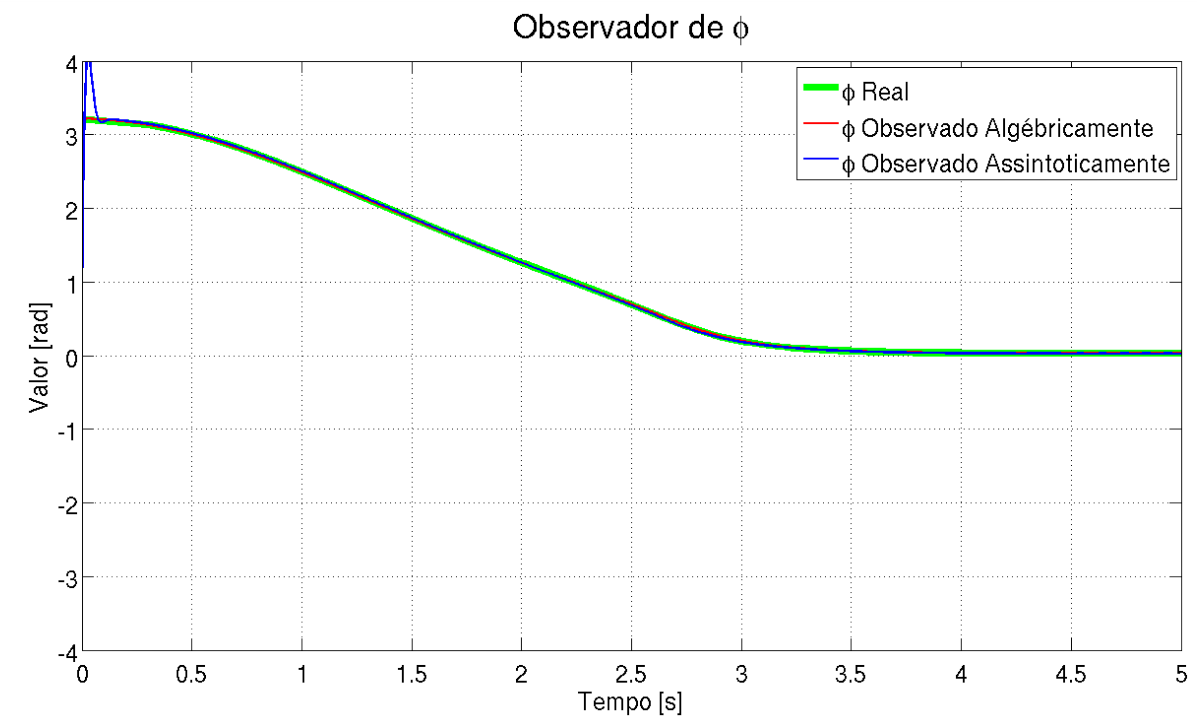

Figura 5.19: Comparativo entre os observadores de estado.

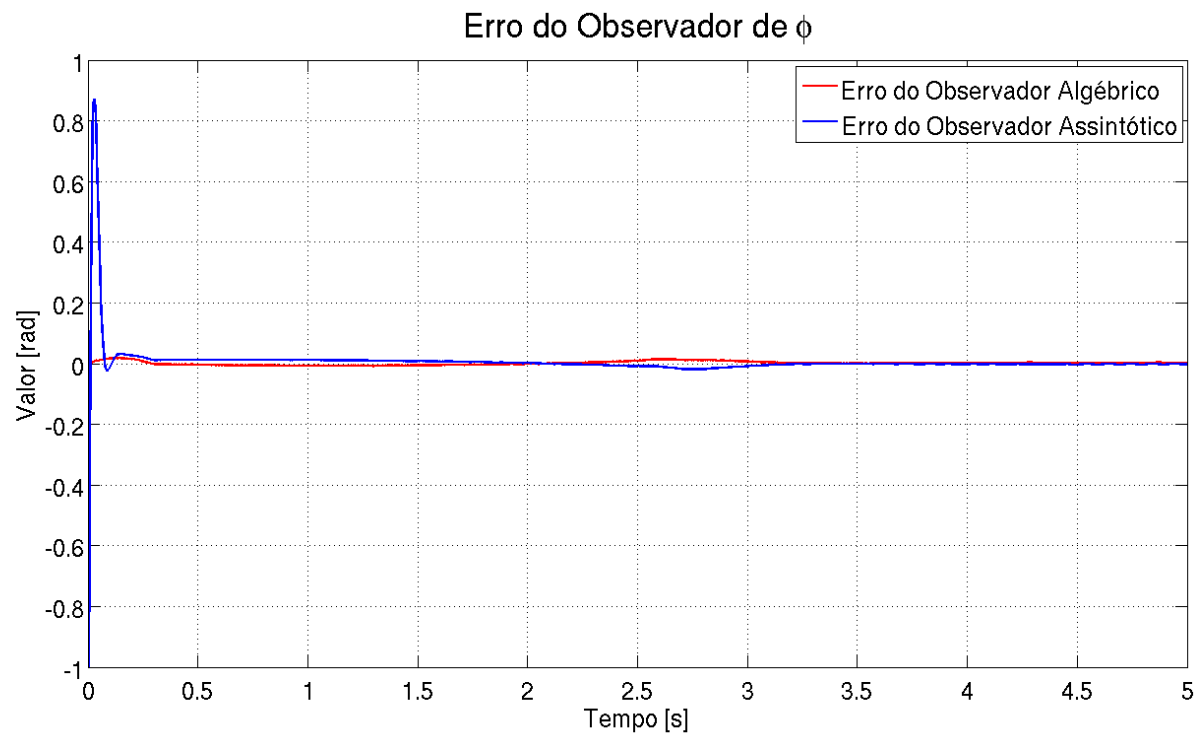

Figura 5.20: Comparativo entre os observadores de estado.

A variável de estado $\phi$ é estimada de maneira muito mais próxima no estimador algébrico. 


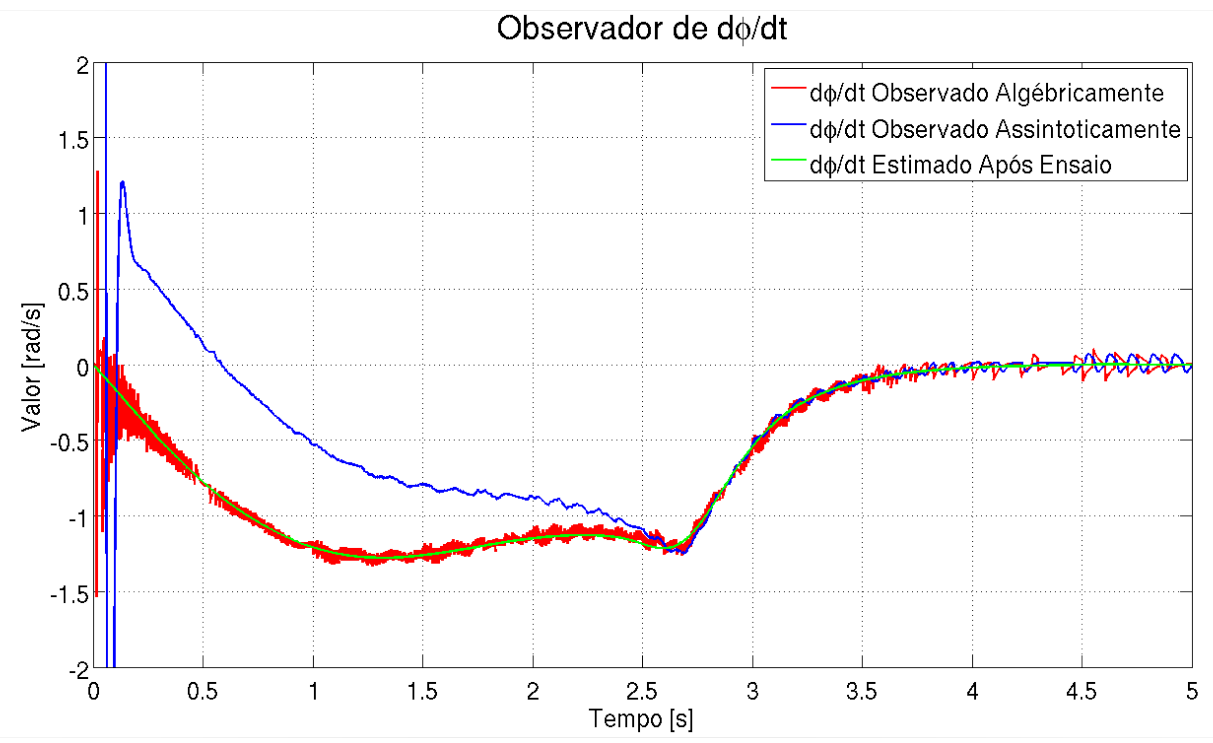

Figura 5.21: Comparativo entre os observadores de estado.

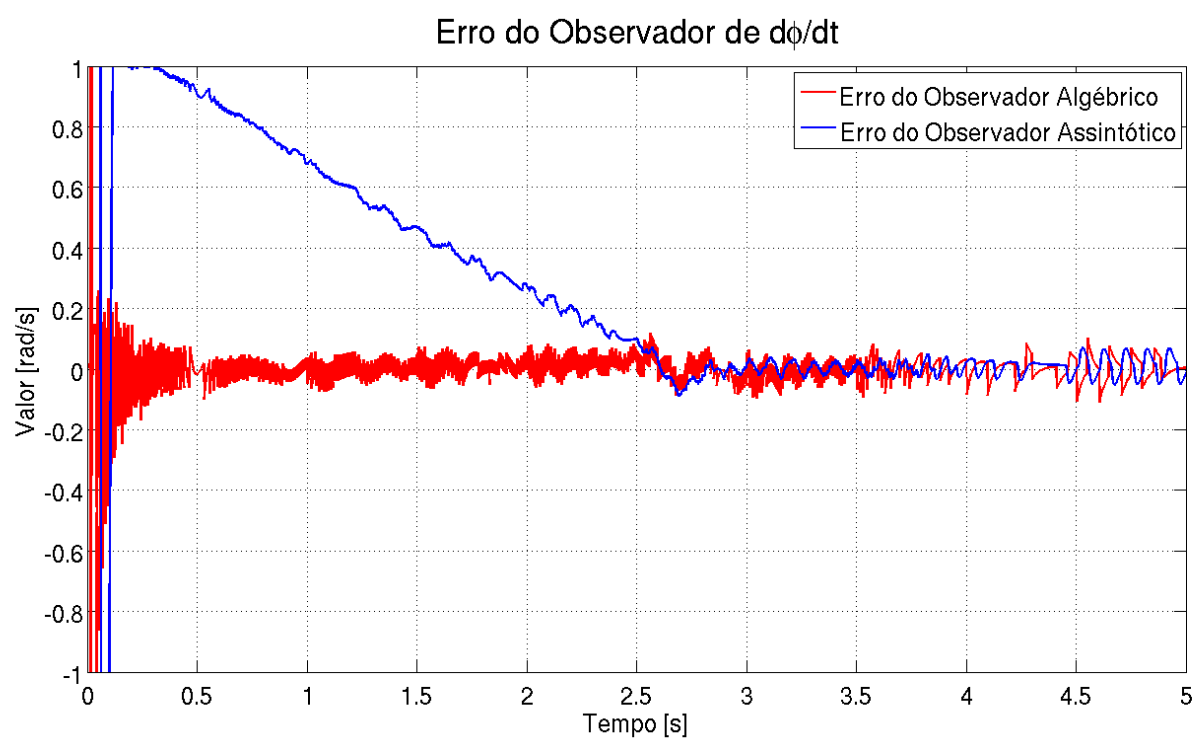

Figura 5.22: Comparativo entre os observadores de estado.

A variável de estado $\dot{\phi}^{1}$ é estimada de maneira muito mais próxima no estimador algébrico, observa-se que a menos do ruído, o erro é sempre muito próximo de zero.

\footnotetext{
${ }^{1} \mathrm{O}$ valor de referência é na realidade uma estimativa feita após ensaio utilizando a técnica de Savitzky-Golay [12]com um polinômio de ordem 3 e uma janela de 299 amostras. Desta maneira, utilizando valores futuros em cálculo efetuado após o ensaio, obtivemos uma estimativa da primeira derivada do sinal medido e comparamos com o observado "on-line" e em tempo real, numa realização puramente causal.
} 


\subsubsection{Comparativo entre os esforços de controle}

Para concluir os comparativos, apresentamos o esforço de controle em cada tipo de abordagem:

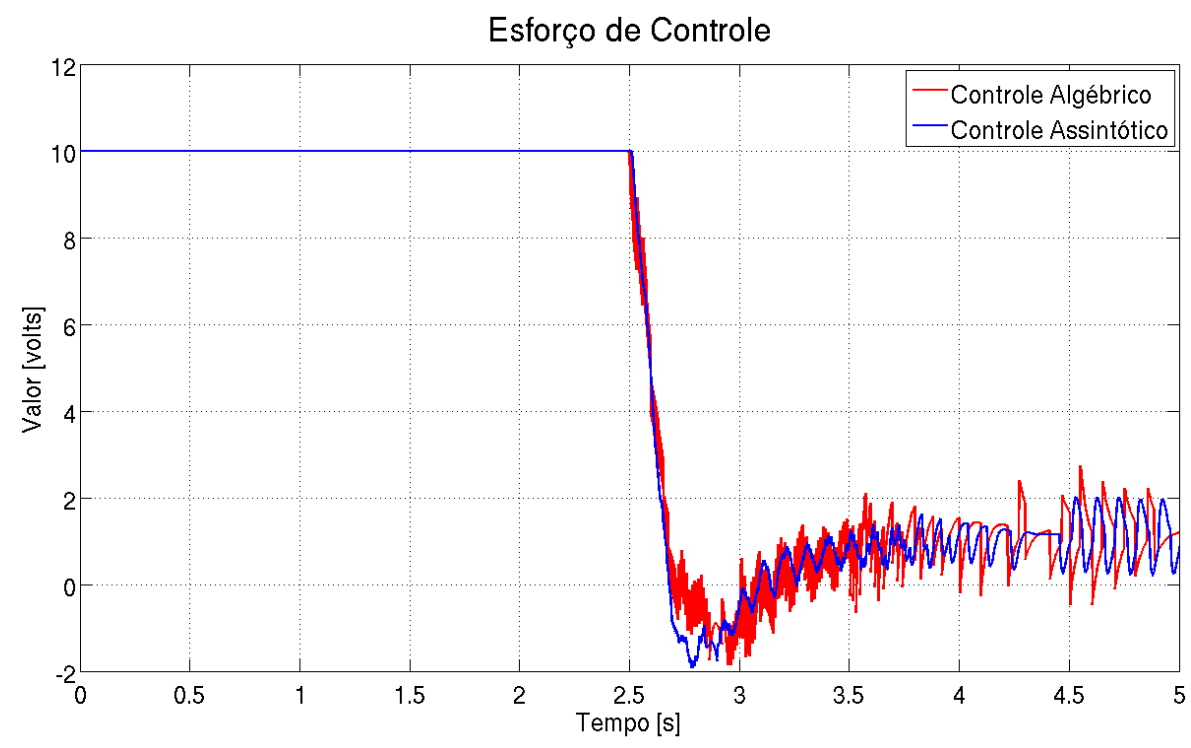

Figura 5.23: Comparativo entre os esforços de controle.

O esforço de controle é praticamente o mesmo em ambas as abordagens.

\subsubsection{Considerações finais sobre o ensaio realizado}

Como metodologia de controle a teoria de estimadores algébricos se mostrou bem eficaz, se comportando tão bem quanto a teoria convencional. O sistema construído apresenta uma não linearidade que não chegou a comprometer o controle linear que bastou para realizar o controle do pêndulo, mas, validou o funcionamento do controlador algébrico construído, de forma que sua utilização pode ser considerada em sistemas com não linearidades mais marcantes.

O comportamento obtido em ensaio foi ligeiramente diferente do obtido nas simulações devido a discrepâncias entre o sistema real e o modelo adotado. Não consideramos, por exemplo, a resistência elétrica do condutor que interliga o circuíto de potência e o motor. Tal condutor foi escolhido fino e flexível para não prejudicar o movimento do pêndulo e em contrapartida, sua resistência elétrica se mostrou não completamente desprezível. Mesmo diante deste e possivelmente de outros erros de modelagem, o resultado de controle foi muito bom, sendo possível posicionar o braço do pêndulo na posição vertical de forma rápida.

Também ficou evidente nos ensaios que o ruído presente não é tão grande quanto o utilizado 
nas simulações em computador. De fato, devido ao uso de um encoder, as únicas fontes de incertezas foram a própria quantização do encoder (comparável à quantização da placa DA/AD) e às trepidações e do sistema mecânico. 


\section{Conclusões e Trabalhos Futuros}

Pelos diversos ensaios e simulações realizadas, concluímos que a teoria de estimadores rápidos é promissora e pode produzir observadores que tem algumas vantagens em relação aos observadores tradicionais. Como vantagens mais evidentes estão a rápida estimação dos estados internos e uma rápida atenuação do ruído de medição. Por outro lado, o ruído resultante ainda é elevado em algumas situações, sendo necessária a escolha correta dos parâmetros do estimador para obter o melhor compromisso entre validade da estimativa e rejeição dos ruídos. No presente momento não existe uma metodologia estabelecida para se encontrar estes parâmetros. Faltam ainda, pesquisas com relação ao correto ajuste do intervalo de reinicialização do estimador, na busca de uma metodologia robusta e que permita melhorar a rejeição de ruídos e também minimizar o erro das derivadas estimadas.

A possibilidade de estimar quase que instantaneamente o estado interno de um sistema não linear nos permite projetar uma lei de controle e construir um controlador sem preocupações com a validade do teorema da separação para a planta em questão. Entretanto esta não foi uma necessidade muito evidente no sistema proposto construído para ensaios em bancada, possivelmente devido à lei de controle utilizada que mascarou em grande parte suas características não lineares.

A maioria das possibilidades abertas para pesquisas futuras são relacionadas à implementação da teoria em tempo discreto, dando assim continuidade ao presente trabalho. Na seção 3.4, menciona-se a possibilidade de utilizar outra técnica para encontrar um polinômio que aproxime as últimas amostras do sinal de entrada. Durante a realização deste trabalho, uma idéia que surgiu foi a utilização de um intervalo com mais amostras do que o mínimo necessário e utilizar um método de mínimos quadrados ponderado para encontrar um polinômio de ordem baixa. Esta implementação diferenciada pode unir as vantagens da teoria de estimadores rápidos e a atenuação de ruídos obtida pelos filtros de Savitzky-Golay [12], por outro lado será introduzido algum atraso de fase.

Em relação ao controle do sistema mecânico construído, uma alternativa mais elaborada 
pode se utilizar da segunda derivada da posição do braço para estimar a velocidade do disco. Desta maneira podemos considerar o modelo completo do motor e assim aplicar a tensão adequada em sua armadura para produzir exatamente o torque necessário, compensando a aproximação feita para o motor operando em baixas velocidades. 


\section{Referências Bibliográficas}

[1] REGER, J.; RAMIREZ, H.; FLIESS, M. On non-asymptotic observation of nonlinear systems. In: Decision and Control, 2005 and 2005 European Control Conference. CDCECC'05. 44th IEEE Conference on. [S.1.: s.n.], 2005. p. 4219-4224.

[2] REGER, J.; MAI, P.; SIRA-RAMIREZ, H. Robust algebraic state estimation of chaotic systems. In: Control Applications, 2006 IEEE International Conference on. [S.1.: s.n.], 2006. p. 326-331.

[3] NOVAES, C. E. d. B.; SILVA, P. S. P. On non-asymptotic state observers and their implementation in discrete time. DINCON'09 - Bauru (8. : 2009 : Bauru) ISBN: 978-85-8688345-3, p. 6, May 2009. Disponível em: < http://carlosnovaes.files.wordpress.com/2009/06/03novaes.pdf $>$.

[4] KHALIL, H. Nonlinear systems. [S.1.]: Prentice Hall Upper Saddle River, NJ, 2002.

[5] KAILATH, T. Linear Systems. [S.1.]: Prentice Hall, 1980. Paperback. ISBN 0135369614.

[6] FLIESS, M.; LAMNABHI, M.; LAMNABHI-LAGARRIGUE, F. An algebraic approach to nonlinear functional expansions. Circuits and Systems, IEEE Transactions on, v. 30, n. 8, p. 554-570, 1983.

[7] FLIESS, M.; SIRA-RAMIREZ, H. An algebraic framework for linear identification. ESAIM CONTROLE OPTIMISATION ET CALCUL DES VARIATIONS, EDP SCIENCES, v. 9, p. 151, 2004.

[8] GIBARU, O. D. L. et al. An error analysis in the algebraic estimation of a noisy sinusoidal signal. 16th Mediterranean Conference on Control and Automation, p. 1296-1301, 2008.

[9] MIKUSINSKI, J. Operational Calculus. [S.1.]: Elsevier, 1960.

[10] MAKKAR, C. et al. A new continuously differentiable friction model for control systems design. Proceedings of the 2005 IEEE/ASME International Conference on Advanced Intelligent Mechatronics, p. 6, July 2005.

[11] KIRKPATRICK, S.; JR., C. D. G.; VECCHI, M. P. Optimization by simulated annealing. Science, v. 220, p. 671-680, May 1983. Disponível em: $<$ http://www.idi.ntnu.no/emner/tdt4/kirkpatrick83optimizationsimulatedannealing.pdf $>$.

[12] SAVITZKY, A.; GOLAY, M. J. E. Smoothing and differentiation of data by simplified least squares procedures. Analytical Chemistry, v. 36, n. 8, p. 1627+, July 1964. 


\section{ANEXO A - Demonstrações}

\section{A.1 Demonstração do resultado sobre integrais de polinômios}

\section{Lema:}

Seja $f\left(t_{0}+\Delta_{t}\right)$ uma função definida como uma integral múltipla:

$$
f\left(t_{0}+\Delta_{t}\right)=\int_{t_{0}}^{t_{0}+\Delta_{t}} \cdots \int \tau^{m} d \tau^{n} t_{0}, \Delta_{t} \in \mathbb{R}^{+} ; m, n \in \mathbb{N}^{+*}
$$

Então, podemos afirmar que:

$$
f\left(t_{0}+\Delta_{t}\right)=\sum_{j=0}^{m} \frac{m !}{j !(m+n-j) !} t_{0}^{j} \Delta_{t}^{m+n-j}
$$

\section{Prova:}

Seja uma nova função $g\left(t_{0}+\Delta_{t}\right)$ definida por:

$$
g\left(t_{0}+\Delta_{t}\right)=\int_{t_{0}}^{t_{0}+\Delta_{t}} f(\tau) d \tau
$$

então:

$$
f\left(t_{0}+\Delta_{t}\right)=\sum_{j=0}^{m} \frac{m !}{j !(m+n-j) !} t_{0}^{j} \Delta_{t}^{m+n-j}
$$

$\mathrm{e}$

$$
g\left(t_{0}+\Delta_{t}\right)=\sum_{j=0}^{m} \frac{m !}{j !(m+n+1-j) !} t_{0}^{j} \Delta_{t}^{m+n+1-j}
$$


mas, $g\left(t_{0}+\Delta_{t}\right)$ também pode ser calculada como se segue:

$$
\begin{gathered}
g\left(t_{0}+\Delta_{t}\right)=\int_{t_{0}}^{t_{0}+\Delta_{t}} f(\tau) d \tau \\
g\left(t_{0}+\Delta_{t}\right)=\int_{t_{0}}^{t_{0}+\Delta_{t}} f\left(t_{0}+\left(\tau-t_{0}\right)\right) d \tau \\
g\left(t_{0}+\Delta_{t}\right)=\int_{t_{0}}^{t_{0}+\Delta_{t}}\left[\sum_{j=0}^{m} \frac{m !}{j !(m+n-j) !} t_{0}^{j}\left(\tau-t_{0}\right)^{m+n-j}\right] d \tau \\
g\left(t_{0}+\Delta_{t}\right)=\sum_{j=0}^{m} \frac{m !}{j !(m+n-j) !} t_{0}^{j}\left[\int_{t_{0}}^{t_{0}+\Delta_{t}}\left(\tau-t_{0}\right)^{m+n-j} d \tau\right] \\
g\left(t_{0}+\Delta_{t}\right)=\sum_{j=0}^{m} \frac{m !}{j !(m+n-j) !} t_{0}^{j}\left[\frac{\left(\tau-t_{0}\right)^{m+n+1-j}}{m+n+1-j}\right]_{t_{0}}^{t_{0}+\Delta_{t}} \\
g\left(t_{0}+\Delta_{t}\right)=\sum_{j=0}^{m} \frac{m !(m+n+1-j) !}{j !} t_{0}^{j} \Delta_{t}^{m+n+1-j}
\end{gathered}
$$

Que é exatamente o mesmo resultado de (A.1).

\section{A.2 Equação do filtro $Z$ em tempo discreto}

A derivada de cada estágio do filtro é uma função do sinal de entrada $(y(t))$, tempo $t$ e, exceto para o último estágio, também uma função do estágio predecessor. Se a entrada puder ser aproximada por um polinômio durante um intervalo de tempo, então poderemos calcular a variação de cada estágio neste intervalo de tempo. De posse do valor anterior de cada estágio do filtro no tempo inicial $t=t_{0}$ de cada amostra, então podemos saber o valor final após este intervalo de amostragem.

Para exemplificar, seja um sinal de entrada aproximado numa dada amostra por:

$$
\widetilde{y}(t)=\alpha_{1} t+\alpha_{2}
$$

Então, o último estágio do filtro $\mathrm{Z}$ será: 


$$
\dot{Z}_{k-1}(t)=B_{k-1}\left[\alpha_{1} t+\alpha_{2}\right] t^{0}
$$

Integrando no intervalo $t_{0}$ e $t_{f}=t_{0}+\Delta_{t}$ :

$$
\begin{aligned}
Z_{k-1}\left(t_{0}+\Delta_{t}\right) & =\int_{t_{0}}^{t_{0}+\Delta_{t}} B_{k-1}\left(\alpha_{1} \tau+\alpha_{2}\right) d \tau+ \\
& +Z_{k-1}\left(t_{0}\right)
\end{aligned}
$$

$$
\begin{aligned}
Z_{k-1}\left(t_{0}+\Delta_{t}\right) & =B_{k-1} \int_{t_{0}}^{t_{0}+\Delta_{t}} \alpha_{1} \tau d \tau+ \\
& +B_{k-1} \int_{t_{0}}^{t_{0}+\Delta_{t}} \alpha_{2} d \tau+ \\
& +Z_{k-1}\left(t_{0}\right)
\end{aligned}
$$

para o penúltimo estágio:

$$
\dot{Z}_{k-2}(t)=B_{k-2}\left[\alpha_{1} t+\alpha_{2}\right] t^{1}+Z_{k-1}(t)
$$

Integrando no intervalo de tempo já definido: 


$$
\begin{aligned}
Z_{k-2}\left(t_{0}+\Delta_{t}\right) & =B_{k-2} \int_{t_{0}}^{t_{0}+\Delta_{t}} \alpha_{1} \tau^{2} d \tau+ \\
& +B_{k-2} \int_{t_{0}}^{t_{0}+\Delta_{t}} \alpha_{2} \tau d \tau+ \\
& +B_{k-1} \int^{t_{0}+\Delta_{t}} \ldots \int \alpha_{1} \tau d \tau^{2}+ \\
& +B_{k-1} \int^{t_{0}+\Delta_{t}} \ldots \int \alpha_{2} d \tau^{2}+ \\
& +\int_{t_{0}}^{t_{0}+\Delta_{t}} Z_{k-1}\left(t_{0}\right) \\
& +Z_{k-2}\left(t_{0}\right)
\end{aligned}
$$

Avançando para os outros estágios, veremos que cada equação é composta pela primeira integral das parcelas deste estágio, a segunda integral das parcelas do estágio seguinte e assim sucessivamente até o último estágio. Além disto, as condições iniciais surgem como constantes de integração no estágio atual e como integrais nos estágios seguintes. Generalizando:

$$
\begin{aligned}
Z_{l}\left(t_{0}+\Delta_{t}\right) & =\sum_{h=1}^{k-l}\left[B_{l-1+h} \sum_{g=0}^{p}\left[\alpha_{g} \int \cdots \int \tau^{k+g-h-l} d \tau^{h}\right]+\right. \\
& \left.+Z_{l+h}\left(t_{0}\right) \int \cdots \int d \tau^{h}\right]+Z_{l}\left(t_{0}\right)
\end{aligned}
$$

Onde $p$ é a ordem do polinômio que aproxima a entrada $y(t)$. Por clareza, os intervalos de integração foram omitidos nesta equação.

Usando o resultado (3.1), escrevemos:

$$
\begin{aligned}
Z_{l}\left(t_{0}+\Delta_{t}\right) & =\sum_{g=0 h=1}^{p} \sum_{h=1}^{k-l}\left[\alpha_{g} \sum_{j=0}^{k+g-h-l} B_{l-1+h} \times\right. \\
& \times \frac{(k+g-h-l) !}{j !(k+g-l-j) !} \Delta_{t}^{k+g-l-j} t_{0}^{j}+ \\
& \left.+\quad Z_{l+h}\left(t_{0}\right) \frac{\Delta_{t}^{h}}{h !}\right]+Z_{l}\left(t_{0}\right)
\end{aligned}
$$


Assim, a equação que define o valor de cada estágio do filtro $\mathrm{Z}$ em um dado intervalo de amostragem é uma combinação linear de produtos de potências inteiras de $\Delta_{t}$ por potências inteiras de $t_{0}$, e então multiplicada por cada coeficiente do polinômio que aproxima as últimas amostras do sinal de entrada. 


\section{ANEXO B - Resumo de Implementação}

Vamos apresentar agora um resumo focado na implementação de estimadores algébricos. A figura B.1 representa o diagrama geral de controle e a figura B.2 na página seguinte ilustra os detalhes construtivos do estimador de derivadas.

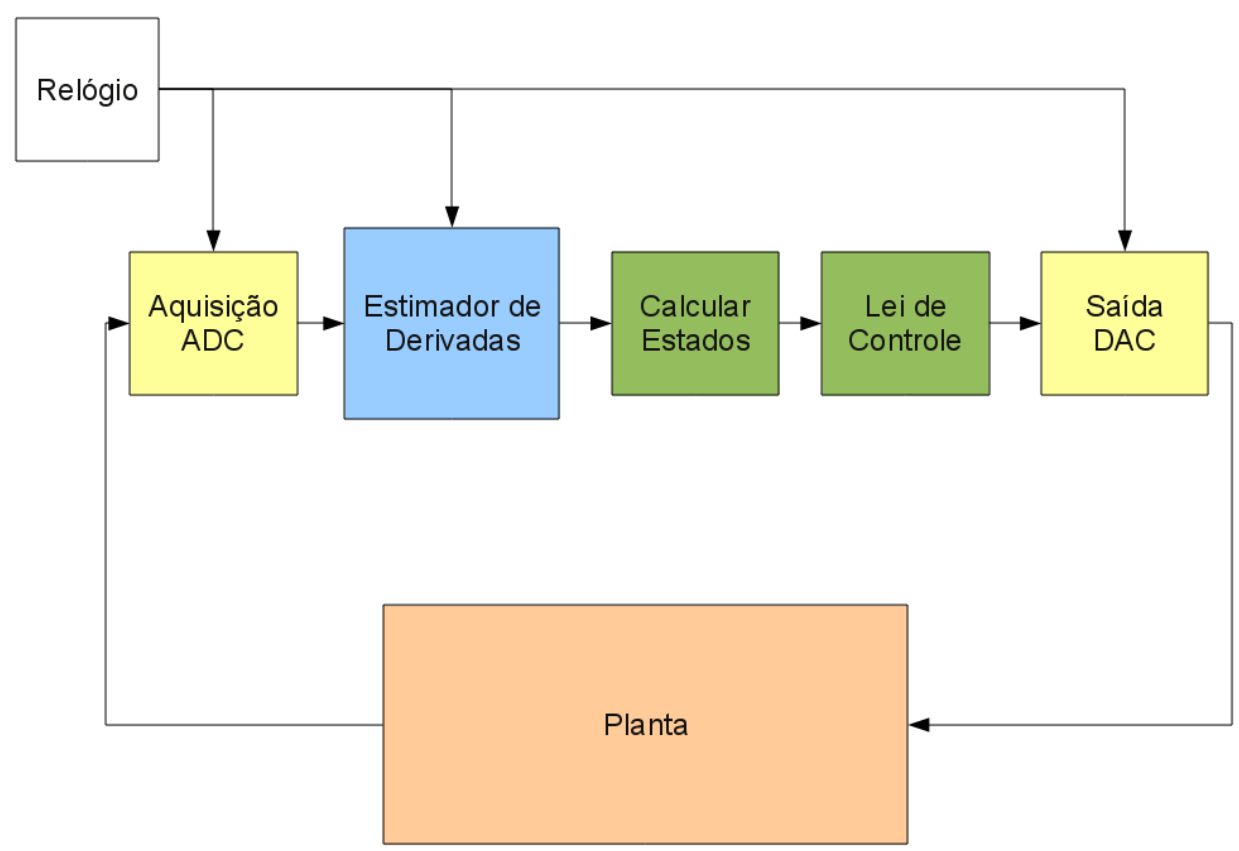

Figura B.1: Esquema fundamental do estimador. 


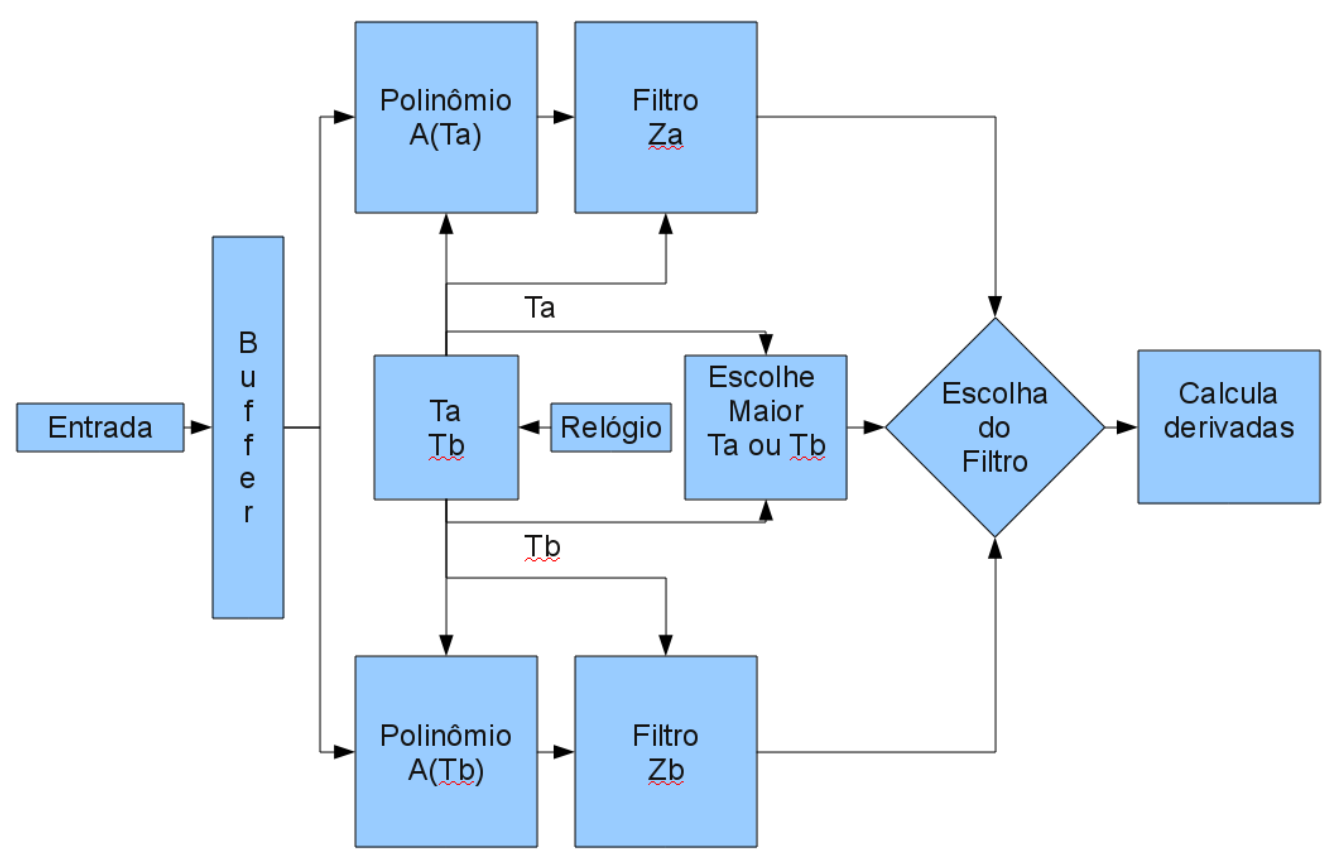

Figura B.2: Esquema detalhado do estimador de derivadas

\section{B.1 Definição dos parâmetros do sistema}

Devemos primeiramente definir alguns parâmetros do projeto. Primeiramente a taxa de amostragem que é determinada pela banda passante do sistema, os outros parâmetros são relativos ao estimador e podem ser ajustados via simulação.

A tabela B.1 apresenta estes parâmetros e uma sugestão preliminar de valor. 
Tabela B.1: Parâmetros do Estimador em tempo discreto.

\begin{tabular}{|c|c|c|c|}
\hline Parâmetro & Descrição & Comportamento & Sugestão \\
\hline$\Delta_{t}$ & $\begin{array}{l}\text { Taxa de amostragem do sinal } \\
\text { cujas derivadas serão estimadas. }\end{array}$ & $\begin{array}{l}\text { Aumentando-se a taxa de } \\
\text { amostragem, aumenta-se a } \\
\text { quantidade de detalhes } \\
\text { capturados. }\end{array}$ & $\begin{array}{l}\text { Dobro da maior freqüência } \\
\text { de interesse. }\end{array}$ \\
\hline$d$ & $\begin{array}{l}\text { Ordem da maior derivada que se } \\
\text { deseja estimar. }\end{array}$ & - & - \\
\hline$k$ & Ordem do estimador. & $\begin{array}{l}\text { Estimadores de ordem maior } \\
\text { produzem estimativas válidas por } \\
\text { tempo maior. Precisa ser superior } \\
\text { a } d .\end{array}$ & $\begin{array}{l}\text { Ao menos } d+1 . \text { Uma boa } \\
\text { região é entre } 5 \text { e } 9 \text { se for } \\
\text { suficiente para atender o } \\
\text { critério anterior. }\end{array}$ \\
\hline$p$ & $\begin{array}{c}\text { Grau do polinômio que aproxima } \\
\text { as amostras de } y(t) .\end{array}$ & $\begin{array}{l}\text { Um grau mais elevado aumenta a } \\
\text { riqueza de detalhes mas diminui a } \\
\text { atenuação do ruído. }\end{array}$ & $\begin{array}{l}1 \text { grau superior à ordem da } \\
\text { maior derivada desejada }(d) .\end{array}$ \\
\hline$t_{\text {reset }}$ & $\begin{array}{l}\text { Intervalo de reinicialização dos } \\
\text { estimadores. }\end{array}$ & $\begin{array}{l}\text { Esta intimamente relacionado à } \\
\text { frequiência máxima do sinal de } \\
\text { entrada, devendo ser reduzido } \\
\text { para reproduzir comportamentos } \\
\text { de frequiencia mais elevada. Este } \\
\text { intervalo determina o } \\
\text { compromisso entre rejeição de } \\
\text { ruídos e estimativas corretas das } \\
\text { derivadas. Se for grande as } \\
\text { derivadas estimadas podem } \\
\text { desviar muito do valor real, se for } \\
\text { pequeno não há atenuação do } \\
\text { ruído. } \\
\text { Se a ordem do estimador é } \\
\text { reduzida, este intervalo de reset } \\
\text { deve ser reduzido para preservar a } \\
\text { validade das estimativas. } \\
\text { Se a ordem da maior derivada } \\
\text { desejada é aumentada, o intervalo } \\
\text { de reset deve ser reduzido. }\end{array}$ & $\begin{array}{c}\text { Deve ser ensaiado em } \\
\text { simulação até se obter o } \\
\text { comportamento desejado. } \\
\text { Como sugestão inicial, } \\
\text { pode-se verificar nos } \\
\text { ensaios apresentados que } \\
\text { para estimar a primeira } \\
\text { derivada de um sinal } \\
y=\text { sin }(t) \text { com um } \\
\text { estimador de ordem } k=7, \text { o } \\
\text { intervalo de reset foi } \\
\text { próximo de } 8 \text { segundos. }\end{array}$ \\
\hline
\end{tabular}




\section{B.2 Pré cálculo dos coeficientes}

Muitos coeficientes podem ser previamente calculados de forma a acelerar o estimador.

\section{B.2.1 Cálculo da matriz de aproximação polinomial para cada instante possível de amostragem}

A matriz que define os coeficientes do polinômio de grau $p$ que aproxima as últimas $p+1$ amostras do sinal de entrada é variante no tempo e deve ser pré calculada para cada instante $t_{0}$ (instante em que ocorre a amostra atual) possível. Determina-se os instantes $t_{0}$ conhecendo-se a taxa de amostragem e o intervalo de reset:

$$
t_{0}=n \Delta_{t}, n \in\left[1,2,3,4, \ldots, \frac{t_{\text {reset }}}{\Delta_{t}}\right]
$$

Trata-se de uma matriz quadrada com dimensão $p+1$ :

$$
\mathbb{A}(t)=\left(\begin{array}{ccccc}
t_{0}^{0} & t_{0}^{1} & t_{0}^{2} & \cdots & t_{0}^{p} \\
\left(t_{0}-\Delta_{t}\right)^{0} & \left(t_{0}-\Delta_{t}\right)^{1} & \left(t_{0}-\Delta_{t}\right)^{2} & \cdots & \left(t_{0}-\Delta_{t}\right)^{p} \\
\left(t_{0}-2 \Delta_{t}\right)^{0} & \left(t_{0}-2 \Delta_{t}\right)^{1} & \left(t_{0}-2 \Delta_{t}\right)^{2} & \cdots & \left(t_{0}-2 \Delta_{t}\right)^{p} \\
\vdots & \vdots & \vdots & \vdots & \vdots \\
\left(t_{0}-p \Delta_{t}\right)^{0} & \left(t_{0}-p \Delta_{t}\right)^{1} & \left(t_{0}-p \Delta_{t}\right)^{2} & \cdots & \left(t_{0}-p \Delta_{t}\right)^{p}
\end{array}\right)^{-1}
$$

\section{B.2.2 Cálculo das constantes do estimador}

A tabela B.2 demonstra as constantes do estimador e como proceder a sua determinação.

Tabela B.2: Coeficientes do estimador.

\begin{tabular}{|c|c|c|}
\hline \hline Identificação & Equação & Comentários \\
\hline \hline$B(k, i, 1)$ & $\left(\begin{array}{c}k \\
i+1\end{array}\right)^{2}(-1)^{-i}(i+1) !$ & Número Inteiro, $i \in[1,2,3, \ldots, k-1]$. \\
\hline$D(k, i, j)$ & $\left(\begin{array}{c}k+i-j-1 \\
i-j\end{array}\right) \frac{(k-j-1) !}{(k-i-1) !}$ & Número Inteiro positivo ou zero, $i, j \in[1,2,3, \ldots, k-1]$. \\
\hline$E(k, i)$ & $\frac{(k+i-1) !}{i !(k-i-1) !}$ & Número Inteiro positivo, $i \in[1,2,3, \ldots, k-1]$. \\
\hline
\end{tabular}




\section{B.3 Construção do estimador}

O estimador proposto é composto na realidade por dois conjuntos de filtros variantes no tempo, operando em paralelo e reinicializados alternadamente. Para tal, devemos então criar dois vetores chamados de $Z a$ e $Z b$, de dimensão $k-1$. Devemos definir também dois contadores de tempo, um para cada conjunto de filtros variantes no tempo e que denominaremos $t a$ e $t b$. Inicialmente, ambos os vetores e seus respectivos contadores de tempo estarão zerados.

As últimas $p+1$ amostras do sinal de entrada devem ser armazenadas, utilizaremos então um vetor de dimensão $p+1$ onde os elementos serão deslocados a cada nova amostra, descartando a amostra mais antiga e inserindo a amostra atual na primeira posição. Denominaremos este vetor como $y_{\text {buffer }}$

\section{B.4 Algorítimo}

O algorítimo descrito a seguir é ilustrado na figura B.3. A cada intervalo de amostragem, seguiremos os seguintes passos:

B.4.1 Ambos os contadores de tempo $(t a$ e $t b)$ serão incrementados de $\Delta_{t}$

$$
\begin{aligned}
& t a=t a+\Delta_{t} \\
& t b=t b+\Delta_{t}
\end{aligned}
$$

\section{B.4.2 Deslocar amostras em $y_{b u f f e r}$ e inserir amostra atual na primeira posição}

$$
\begin{aligned}
& y_{\text {buffer }}\left(t_{0}-\Delta_{t}\right)=\left[\begin{array}{lllll}
y_{5} & y_{4} & y_{3} & y_{2} & y_{1}
\end{array}\right]^{\prime}
\end{aligned}
$$

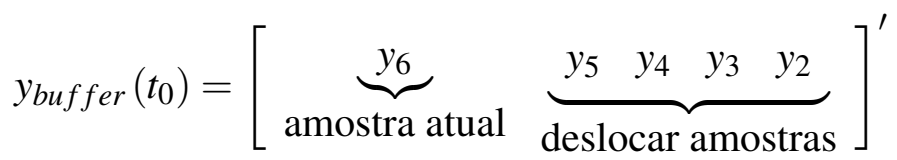


B.4.3 Determinar os coeficientes $\alpha$ para o filtro $Z a$

$$
\alpha=\mathbb{A}(t a) \times y_{\text {buffer }}
$$

B.4.4 Determinar a evolução dos estágios do filtro $Z a$

$$
\begin{aligned}
Z_{l}\left(t_{a}\right) & =\quad \sum_{g=0 h=1}^{p} \sum_{j}^{k-l}\left[\alpha_{g} \sum_{j=0}^{k+g-h-l} B_{l-1+h} \times\right. \\
& \times \frac{(k+g-h-l) !}{j !(k+g-l-j) !} \Delta_{t}^{k+g-l-j}\left(t_{a}-\Delta_{t}\right)^{j}+ \\
& \left.+\quad Z_{l+h}\left(t_{a}-\Delta_{t}\right) \frac{\Delta_{t}^{h}}{h !}\right]+Z_{l}\left(t_{a}-\Delta_{t}\right)
\end{aligned}
$$

B.4.5 Determinar os coeficientes $\alpha$ para o filtro $Z b$

$$
\alpha=\mathbb{A}(t b) \times y_{\text {buffer }}
$$

B.4.6 Determinar a evolução dos estágios do filtro $Z b$

$$
\begin{aligned}
Z_{l}\left(t_{b}\right) & =\quad \sum_{g=0 h=1}^{p} \sum_{j=0}^{k-l}\left[\alpha_{g} \sum_{j=0}^{k+g-h-l} B_{l-1+h} \times\right. \\
& \times \frac{(k+g-h-l) !}{j !(k+g-l-j) !} \Delta_{t}^{k+g-l-j}\left(t_{b}-\Delta_{t}\right)^{j}+ \\
& \left.+\quad Z_{l+h}\left(t_{b}-\Delta_{t}\right) \frac{\Delta_{t}^{h}}{h !}\right]+Z_{l}\left(t_{b}-\Delta_{t}\right)
\end{aligned}
$$

B.4.7 Escolher os estágios do filtro cujo tempo relativo ( $t a$ ou $t b$ ) seja maior e usar estes valores no cálculo das estimativas das derivadas

$$
\tilde{y}^{(i)}\left(t_{0}\right)=E(k, i) \frac{1}{t[a, b]^{i}} y(t[a, b])+\sum_{j=1}^{i} D(k, i, j) \frac{1}{t[a, b]^{k+i-j}} Z[a, b]_{j}(k, t[a, b])
$$


B.4.8 Se o contador de tempo $t a$ tiver valor igual a metade do valor $t_{\text {reset }}$, reinicializar o vetor $Z b$ e o contador $t b$. Se isto não ocorrer, então verificar se o contador $t b$ tem valor igual ou superior a metade do valor $t_{\text {reset }}$ e neste caso reinicializar o vetor $Z a$ e o contador $t a$. Esta política implementa o procedimento de reinicialização alternada ilustrado em 2.1

\section{B.4.9 Prosseguir com a próxima amostra}

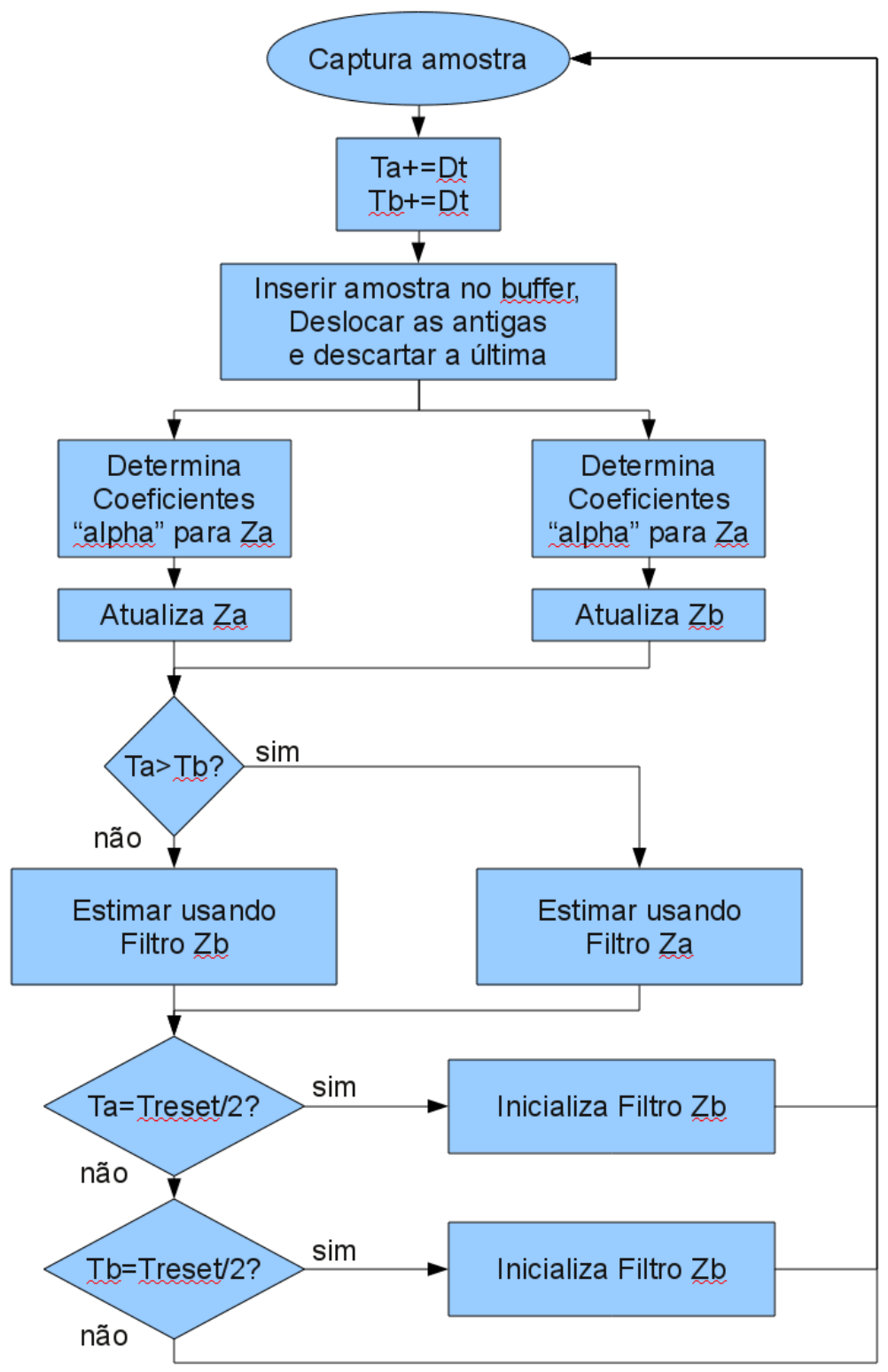

Figura B.3: Fluxograma do algorítimo. 


\section{ANEXO C - Ferramentas utilizadas}

\section{C.1 Introdução}

No desenvolvimento do presente trabalho, utilizou-se de diversos softwares e linguagens de programação. As simulações foram executadas em MATLAB. Os desenhos foram feitos utilizando o software PovRay e as peças usinadas na construção do pêndulo foram desenhadas com auxílio do software VariCAD. Os programas em linguagem $\mathrm{C} / \mathrm{C}++$ foram compilados em ambiente GNU/Linux com o software GCC (GNU Compiler Colection) e o firmware escrito para o microcontrolador PIC foi compilado utilizando a suíte GPUtils (GNU PIC Utilities).

O ensaio em laboratório utilizou alguns componentes de hardware, uma placa de aquisição de dados LYNX modelo CAD12/36, e um computador pessoal PC AMD K6II 300MHz dotado de 32MB de memória RAM. A construção do pêndulo propriamente utilizou um encoder com capacidade para discretizar uma volta completa em 4000 pulsos, e um circuito feito com um microcontrolador PIC foi utilizado para converter os pulsos do encoder (quadratura) em código binário para aquisição pela placa LYNX.

\section{C.2 Programas para ambiente MATLAB}

\section{C.2.1 Programa Makkar_Solve.m}

Este programa encontra os parâmetros para o modelo de atrito de Makkar com base nas informações fornecidas. A busca é feita utilizando-se um processo inspirado em simulação de recozimento (annealing).

function [ Gamma ] = Makkar_Solve( Ff, Vf )

\%MAKKAR_SOLVE Return a Vector of constants for makkar friction model

$\%$ Detailed explanation goes here 


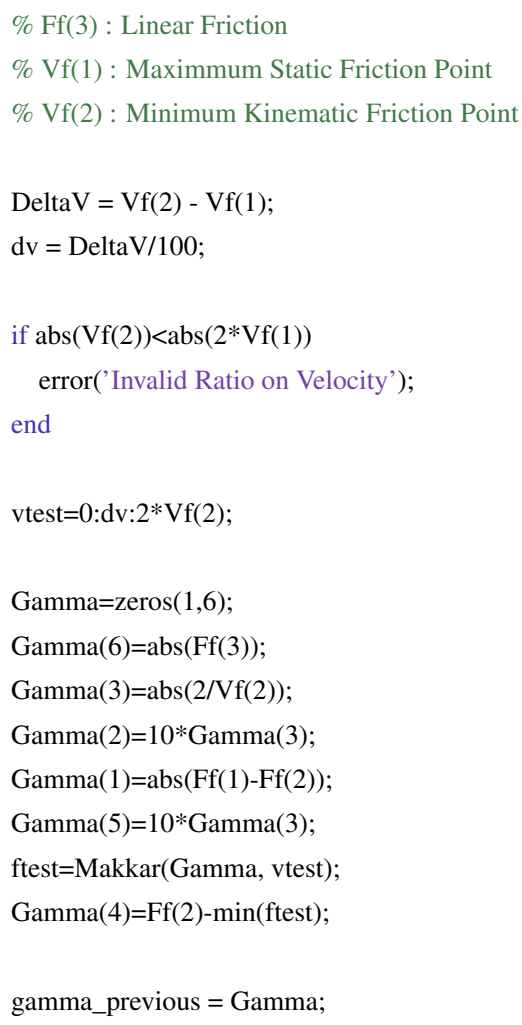




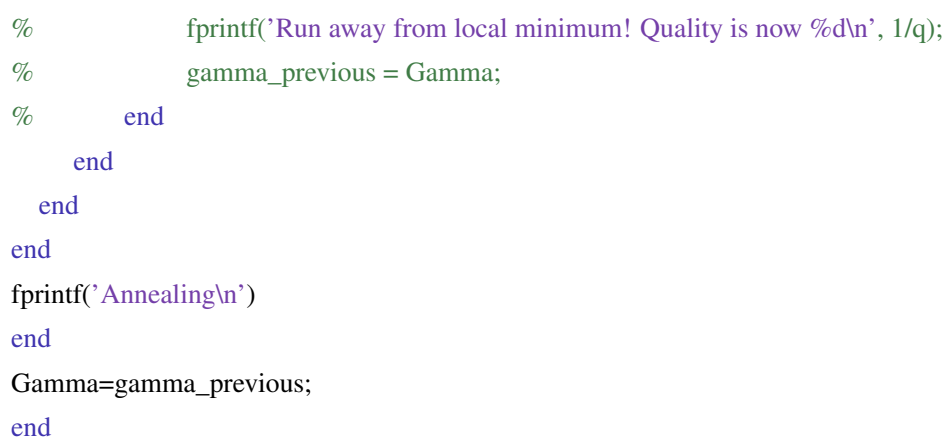

end

function val = MakkarQualityTest $(\mathrm{Ff}, \mathrm{Vf}, \mathrm{Fc}, \mathrm{Vc}$, distant_force, desired_distant_force $)$ $\mathrm{val}=((\operatorname{Ff}(1)-\mathrm{Fc}(1)) / \mathrm{Fc}(1))^{\wedge} 2+((\mathrm{Ff}(2)-\mathrm{Fc}(2)) / \mathrm{Fc}(2))^{\wedge} 2+0.5^{*}((\mathrm{Vf}(1)-\mathrm{Vc}(1)) / \mathrm{Vc}(1))^{\wedge} 2+0.5^{*}((\mathrm{Vf}(2)-\mathrm{Vc}(2)) / \mathrm{Vc}(2))^{\wedge} 2+\left(\left(\operatorname{distant} \_\right.\right.$forcedesired_distant_force)/desired_distant_force $)^{\wedge} 2$; end

function $[$ Force $]=$ Makkar $($ Gamma, Vel)

Force $=\operatorname{Gamma}(1) *(\tanh (\operatorname{Gamma}(2) * \mathrm{Vel})-\tanh (\mathrm{Gamma}(3) * \mathrm{Vel}))+\operatorname{Gamma}(4) * \tanh (\mathrm{Gamma}(5) * \mathrm{Vel})+\mathrm{Gamma}(6) * \mathrm{Vel}$; end

\section{C.2.2 Programa NAO_disc_sfunc.m}

Programa MATLAB LEVEL 2 para implementar um bloco no simulink capaz de realizar a teoria de estimação de derivadas em tempo discreto, da forma proposta no presente trabalho. 
\% Level-2 M file S-Function for Non Assymptotic Observation

\% Revision : 0.2

setup(block);

$\%$ endfunction

function setup(block)

$\%$ Estimator Order

$\%$ Input Approximation Order (2)

$\%$ Reset Interval

$\%$ SampleTime

block.NumDialogPrms $=4$;

$\% \%$ Register number of input and output ports

block.NumInputPorts $=1$;

block.NumOutputPorts = 1;

$\% \%$ Setup functional port properties to dynamically

$\% \%$ inherited.

block.SetPreCompInpPortInfoToDynamic;

block.SetPreCompOutPortInfoToDynamic;

$\%$ Input Dimension is 1

block.InputPort(1).Dimensions $\quad=1$;

block.InputPort(1).DirectFeedthrough = true;

$\%$ Output Dimension is Order - 1

block.OutputPort(1).Dimensions = block.DialogPrm(1).Data-1;

$\% \%$ Set block sample time

block.SampleTimes $=[$ block.DialogPrm(4).Data 0];

$\% \%$ Register methods

block.RegBlockMethod('PostPropagationSetup', @DoPostPropSetup);

block.RegBlockMethod('InitializeConditions', @InitConditions);

block.RegBlockMethod('Outputs', @ @Output);

block.RegBlockMethod('Update', @ @Update);

\%endfunction

function DoPostPropSetup(block)

$\% \%$ Setup Dwork

$\%$ Discrete State Z (1) $\mathrm{Z}$

$\%$ Discrete Input (1) $\mathrm{x}$

$\%$ Reset Time (1) $\mathrm{x}$

$\%$ E Constants (1) $\mathrm{x}$

$\%$ D Constants (1) $\mathrm{x}$

$\%$ Integration Constants (1) $\mathrm{x}$

$\%$ Input Approximation Constants (p)x

$\%$ Control DWork

block.NumDworks $=8$;

$\%$ Discrete State ' $Z$ '

block. Dwork(1).Name = 'Zdisc'; 


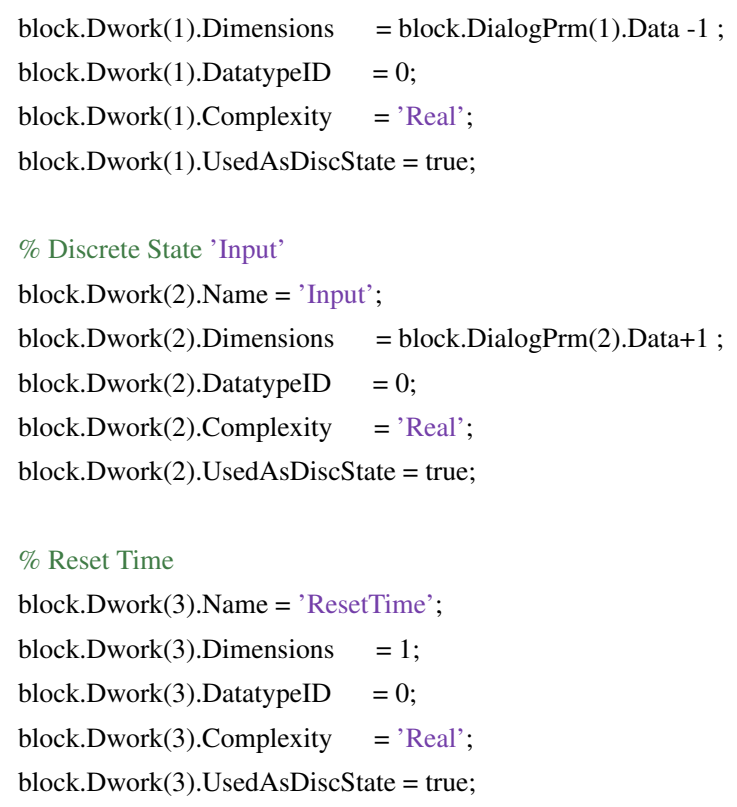




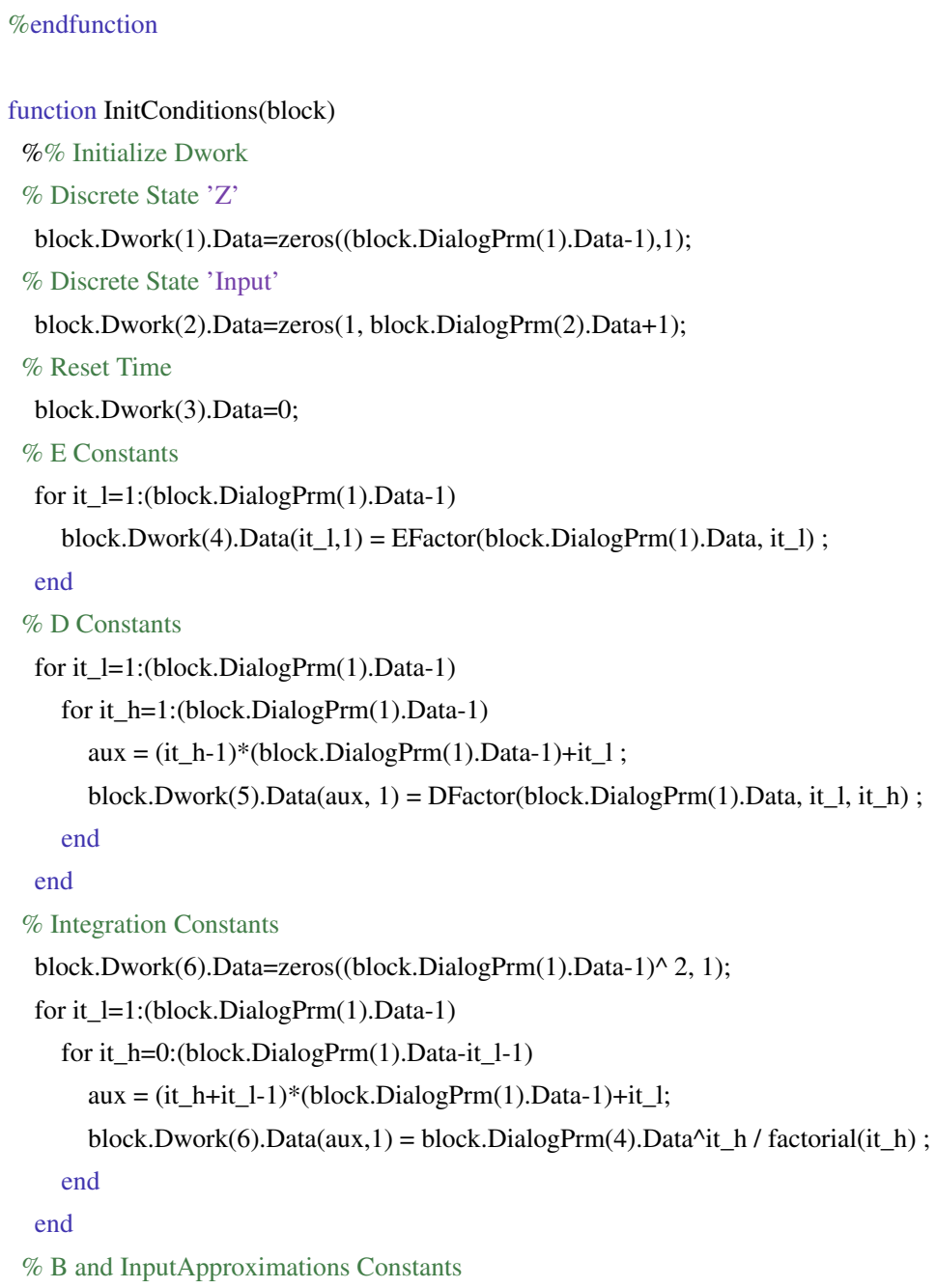




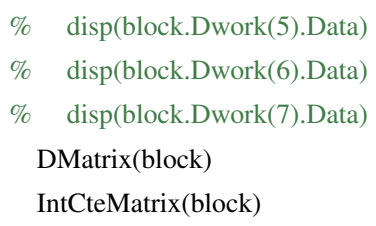

for it_g=0:block.DialogPrm(2).Data

disp(sprintf('Matrix for coefficient Alpha\%d',it_g));

InpApproxMatrix(block,it_g)

end

\%endfunction

function Output(block)

$\%$ disp(block.CurrentTime);

$\%$ Update Discrete Input

block.Dwork(2).Data(1:block.DialogPrm(2).Data, 1) = block.Dwork(2).Data(2:block.DialogPrm(2).Data+1, 1);

block.Dwork(2).Data(block.DialogPrm(2).Data+1) = block.InputPort(1).Data ;

if block.Dwork(8).Data $<0$

block.Dwork(8).Data = block.Dwork(8).Data +1 ;

block.Dwork(3).Data = block.CurrentTime ;

end

if block.CurrentTime-block.Dwork(3).Data >= block.DialogPrm(3).Data

block.Dwork(1).Data $=0 *$ block.Dwork(1).Data ;

block.Dwork(3).Data = block.CurrentTime ;

end

$\%$ Input Approximation Coefficients

times = block.CurrentTime - block.Dwork(3).Data + (-block.DialogPrm(2).Data:0) ${ }^{*}$ block.DialogPrm(4).Data ;

$\%$ disp(times);

$\%$ disp(block.Dwork(2).Data);

$\% \quad$ [coefs, scoefs, mucoefs]=polyfit(times,block.Dwork(2).Data,block.DialogPrm(2).Data);

$\% \quad$ coefs=polyfit(times, block.Dwork(2).Data,block.DialogPrm(2).Data);

coefs=mypolyfit(times',block.Dwork(2).Data');

$\%$ disp(coefs);

if times(block.DialogPrm(2).Data) $>=-0.5 *$ block.DialogPrm(4).Data

$\% \quad \operatorname{disp}($ 'Updating');

tzero_power $=$ times(block.DialogPrm(2).Data).$^{\wedge}(0:$ block.DialogPrm(1).Data+block.DialogPrm(2).Data-2)';

Z_new $=0 *$ block.Dwork(1).Data ;

for it_g=0:block.DialogPrm(2).Data

$\mathrm{mtz}=$ InpApproxMatrix $($ block, it_g) ;

Z_new $=$ Z_new + coefs(block.DialogPrm(2).Data+1-it_g) * mtz * tzero_power ;

end

Z_new $=$ Z_new + IntCteMatrix(block) $*$ block.Dwork(1).Data;

block.Dwork(1).Data $=Z \_$new ;

t_power = times(block.DialogPrm(2).Data+1).$^{\wedge}\left(1: 2^{*}\right.$ block.DialogPrm(1).Data-2)’ ;

$\%$ Derivatives_new $=0 *$ block.OutputPort(1).Data;

Derivatives_new = block.Dwork(4).Data ./ t_power(1:block.DialogPrm(1).Data-1)* block.InputPort(1).Data ;

$\mathrm{mtz}=$ DMatrix $($ block$)$;

for it_j=1:(block.DialogPrm(1).Data-1)

$\% \quad \operatorname{disp}(\operatorname{mtz}(:$, it_j $))$; 
$\% \quad$ disp(t_power(block.DialogPrm(1).Data-it_j+1:2*block.DialogPrm(1).Data-1-it_j));

Derivatives_new $=$ Derivatives_new $+\operatorname{mtz}\left(:, i t \_j\right) . / t \_p o w e r\left(b \operatorname{lock} \cdot \operatorname{Dialog} \operatorname{Prm}(1) \cdot \operatorname{Data}-i t \_j+1: 2 *\right.$ block.DialogPrm(1).Data-1-it_j $) *$ block.Dwork(1).Data(it_j,1) ;

end

block.OutputPort(1).Data = Derivatives_new ;

end

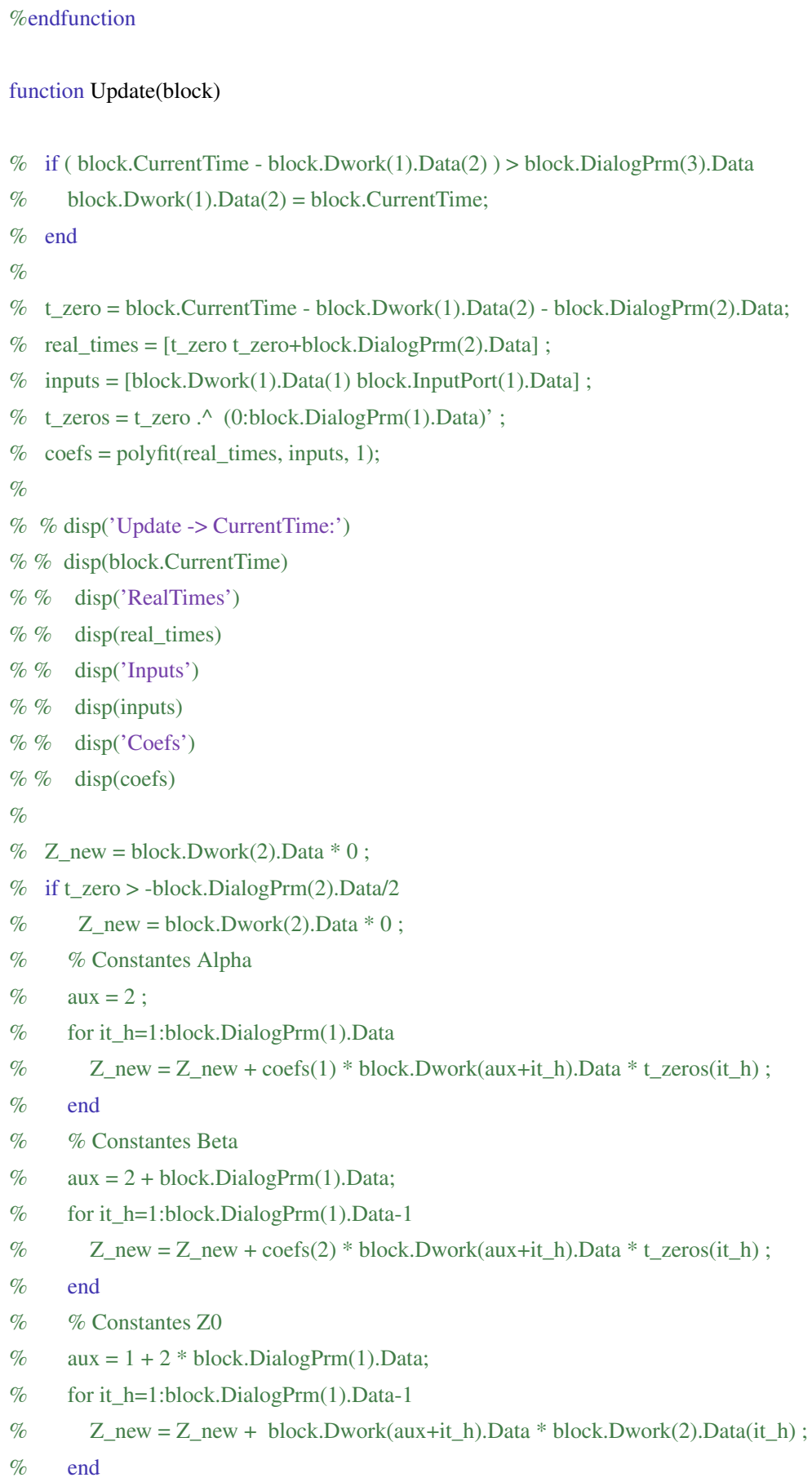




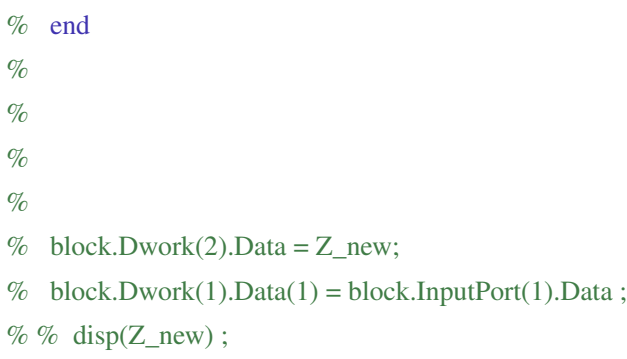




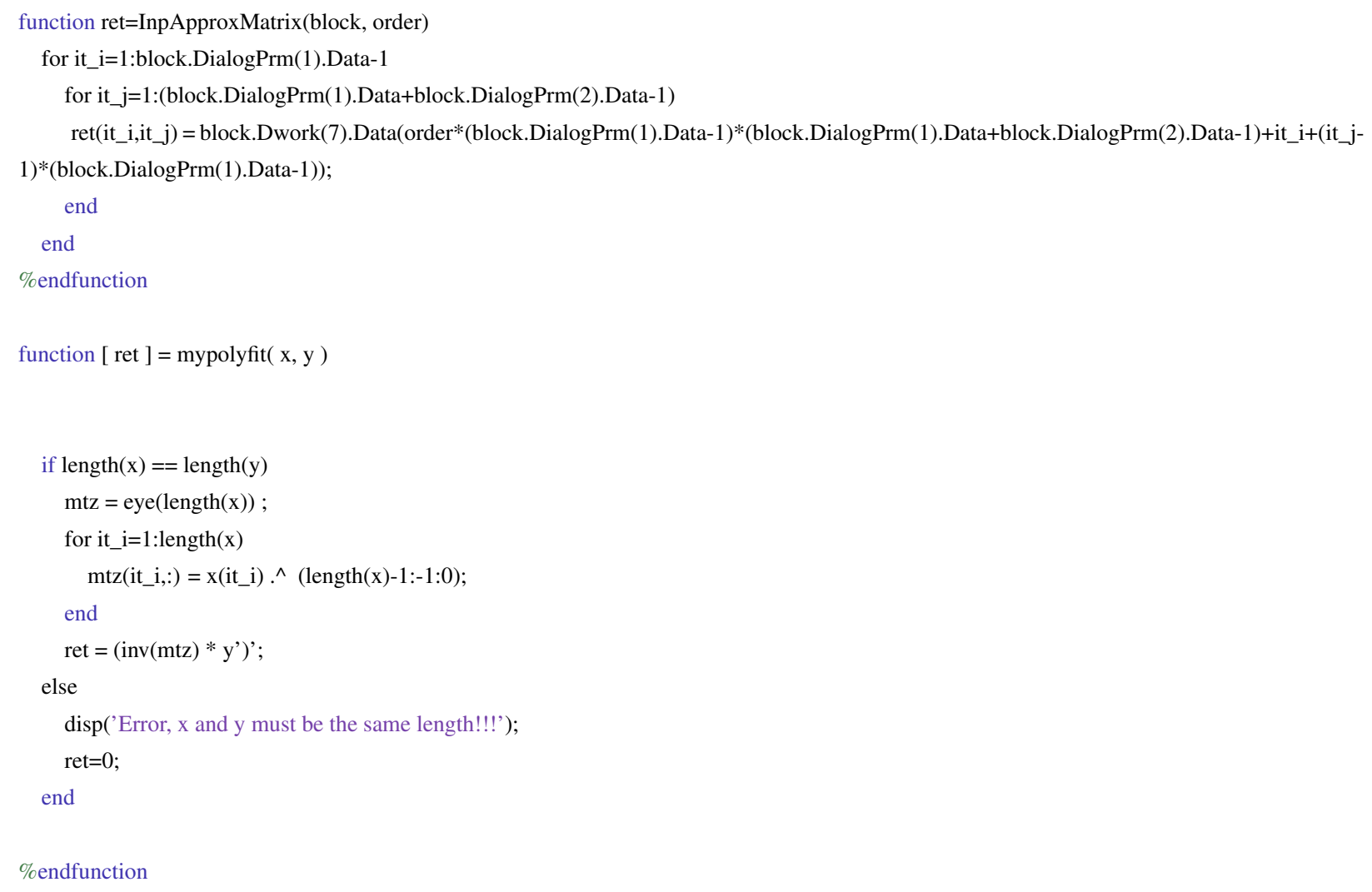

\section{C.2.3 Programa SGDerivation.m}

Programa que utiliza filtros de Savitzky-Golay para obter derivadas suaves de um sinal ruidoso.

function [ rety, rett ] = SGDerivation( $y, d t$, poly_order, window_size, deriv_order )

$\%$ SGDERIVATION Summary of this function goes here

$\%$ Detailed explanation goes here

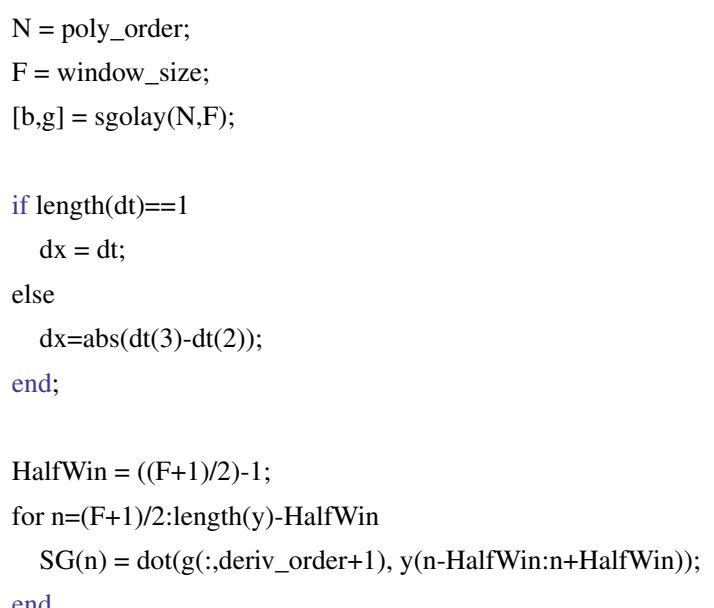


rety $=\mathrm{SG} /(\mathrm{dx} \wedge$ (deriv_order) $)$;

rett $=(1$ length $(\mathrm{y})$-HalfWin $) * d x$;

end

\section{C.2.4 Programa Parametros.m}

Introduz os parâmetros do pêndulo, calcula as matrizes do sistema linear e os ganhos do controlador e observador.

$\begin{array}{lc}\text { g }=9.8 ; & \% \text { Gravity } 9.8 \mathrm{~m} / \mathrm{s}^{\wedge} 2 \\ \text { eta }=0.035 ; & \% \mathrm{rad} \\ \text { L_BAR1 }=0.2 ; & \% \mathrm{~m} \\ \text { L_BAR2 }=0.1 ; & \% \mathrm{~m} \\ \text { M_BAR1 }=0.141 ; & \% \mathrm{Kg} \\ \text { M_BAR2 }=0.0799 ; & \% \mathrm{Kg} \\ \text { M_MOT }=0.31 ; & \% \mathrm{Kg} \\ \text { M_CIL }=0.546 ; & \% \mathrm{Kg} \\ \text { M_DIS }=0.458 ; & \% \mathrm{Kg} \\ \text { J_BAR1 }=0.00229 ; & \% \mathrm{Kg} \cdot \mathrm{m}^{\wedge} 2 \\ \text { J_BAR2 }=0.000422 ; & \% \mathrm{Kg} \cdot \mathrm{m}^{\wedge} 2 \\ \text { J_MOT }=0.0125 ; & \% \mathrm{Kg} \cdot \mathrm{m}^{\wedge} 2 \\ \text { J_CIL }=0.00544 ; & \% \mathrm{Kg} \cdot \mathrm{m}^{\wedge} 2 \\ \text { J_DIS }=0.00289 ; & \% \mathrm{Kg} . \mathrm{m}^{\wedge} 2 \\ \text { VEL_MOT }=270.177 ; & \% \mathrm{rad} / \mathrm{s} \\ \text { K_MOT }=0.0265 ; & \% \mathrm{~N} / \mathrm{A} \\ \text { Ra_MOT }=1.94 ; & \% \mathrm{Ohm} \\ \text { Va_MOT_min }=0.8 ; & \% \mathrm{Volt} \\ \text { Va_MOT_max }=12.0 ; & \% \text { Volt } \\ \text { Phi_MAX_ate }=0.03 * 2 * \mathrm{pi} ; & \% \text { rad }\end{array}$

$\mathrm{C} 1=1 /\left(\mathrm{J} \_\mathrm{CIL}+\mathrm{J} \_\mathrm{BAR} 1+\mathrm{J} \_\mathrm{BAR} 2+\mathrm{J} \_\mathrm{MOT}+\mathrm{L} \_\mathrm{BAR} 1{ }^{\wedge} 2 * \mathrm{M} \_\mathrm{DIS}\right)$;

$\mathrm{C} 2=1 / \mathrm{J} \_$DIS

$\mathrm{C} 3=-\mathrm{g} * \sin ($ eta $) *\left(\right.$ L_BAR2 $*\left(\mathrm{M} \_\mathrm{CIL}+\mathrm{M} \_\right.$BAR2/2) - L_BAR $1 *\left(\mathrm{M} \_\right.$BAR1/2 + M_MOT + M_DIS $)$);

$\%$

$\%$ Tau_MOT_ate $=$ Va_MOT_min $/$ Ra_MOT $*$ K_MOT;

$\%$ Tau_MOT_atc $=$ Tau_MOT_ate $/ 5$;

$\%$ Tau_MOT_atv $=(($ Va_MOT_max - VEL_MOT*K_MOT) / Ra_MOT * K_MOT - Tau_MOT_atc $) /$ VEL_MOT;

\% Gamma_MOT = Makkar_Solve([Tau_MOT_ate Tau_MOT_atc Tau_MOT_atv], [2 10]*1e-4 );

$\%$ save Gamma_MOT.mat Gamma_MOT

load Gamma_MOT.mat

$\%$ Tau_BAR_ate $=$ C $3 * \sin ($ Phi_MAX_ate $)$;

$\%$ [temp]=IdentificaAtritoBarra('livre_1_ccw', C1, C2, C3);

$\%$ Tau_BAR_atv=temp $(1)$; 
$\%$ Tau_BAR_atc=temp $(2)$;

$\%$ Gamma_BAR $=$ Makkar_Solve([Tau_BAR_ate Tau_BAR_atc Tau_BAR_atv], [2 10]*1e-3 );

$\%$ save Gamma_BAR.mat Gamma_BAR Tau_BAR_ate Tau_BAR_atc Tau_BAR_atv

load Gamma_BAR.mat

Phi_0=pi;

Theta_0 $=0$;

Initial $=[$ Theta_0 0 Phi_0 0];

$\%$ Modelo linearizado simples

$\% \mathrm{x}=[$ Phi Phip Thetap ]

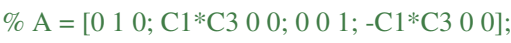

$\% \mathrm{~B}=[0 ;-\mathrm{C} 1 ; \mathrm{C} 1+\mathrm{C} 2]$;

$\% \mathrm{C}=\left[\begin{array}{lll}1 & 0 & 0\end{array}\right] ;$

$\% \mathrm{D}=[0]$;

$\%$ Modelo linearizado com motor

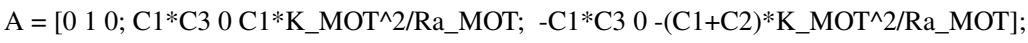

$\mathrm{B}=\left[0 ;-\mathrm{C} 1 * \mathrm{~K} \_\mathrm{MOT} / \mathrm{Ra} \_\mathrm{MOT} ;(\mathrm{C} 1+\mathrm{C} 2)^{*} \mathrm{~K} \_\mathrm{MOT} / \mathrm{Ra} \_\mathrm{MOT}\right]$;

$\mathrm{C}=\left[\begin{array}{lll}1 & 0 & 0\end{array}\right]$;

$\mathrm{D}=[0]$

$\%$ Controle

$\mathrm{G}=\operatorname{place}\left(\mathrm{A}, \mathrm{B},[-2-3+3 \mathrm{i}-3-3 \mathrm{i}]^{*} 1\right)$;

Greal $=[\mathrm{G}(1) \mathrm{G}(2) \mathrm{G}(3)]$;

$\operatorname{eig}(\mathrm{A}-\mathrm{B} * \mathrm{Greal})$

\section{C.2.5 Programa IdentificaAtritoBarra.m}

Tenta identificar as características dos atritos do sistema construído, com base em um ensaio de movimentação livre.

function $[\mathrm{cfat}]=$ IdentificaAtritoBarra(filename)

load(filename)

$[q 0, t]=S G D e r i v a t i o n(\exp (:, 2), \exp (:, 1), 4,499,0)$;

$[\mathrm{q} 1, \mathrm{t}]=$ SGDerivation $(\exp (:, 2), \exp (:, 1), 4,499,1)$;

$[\mathrm{q} 2, \mathrm{t}]=$ SGDerivation $(\exp (:, 2), \exp (:, 1), 4,499,2)$;

set(0,'defaultaxesfontsize',18); 


\section{C.3 Modelos para SIMULINK.}

\section{C.3.1 Modelo Global}

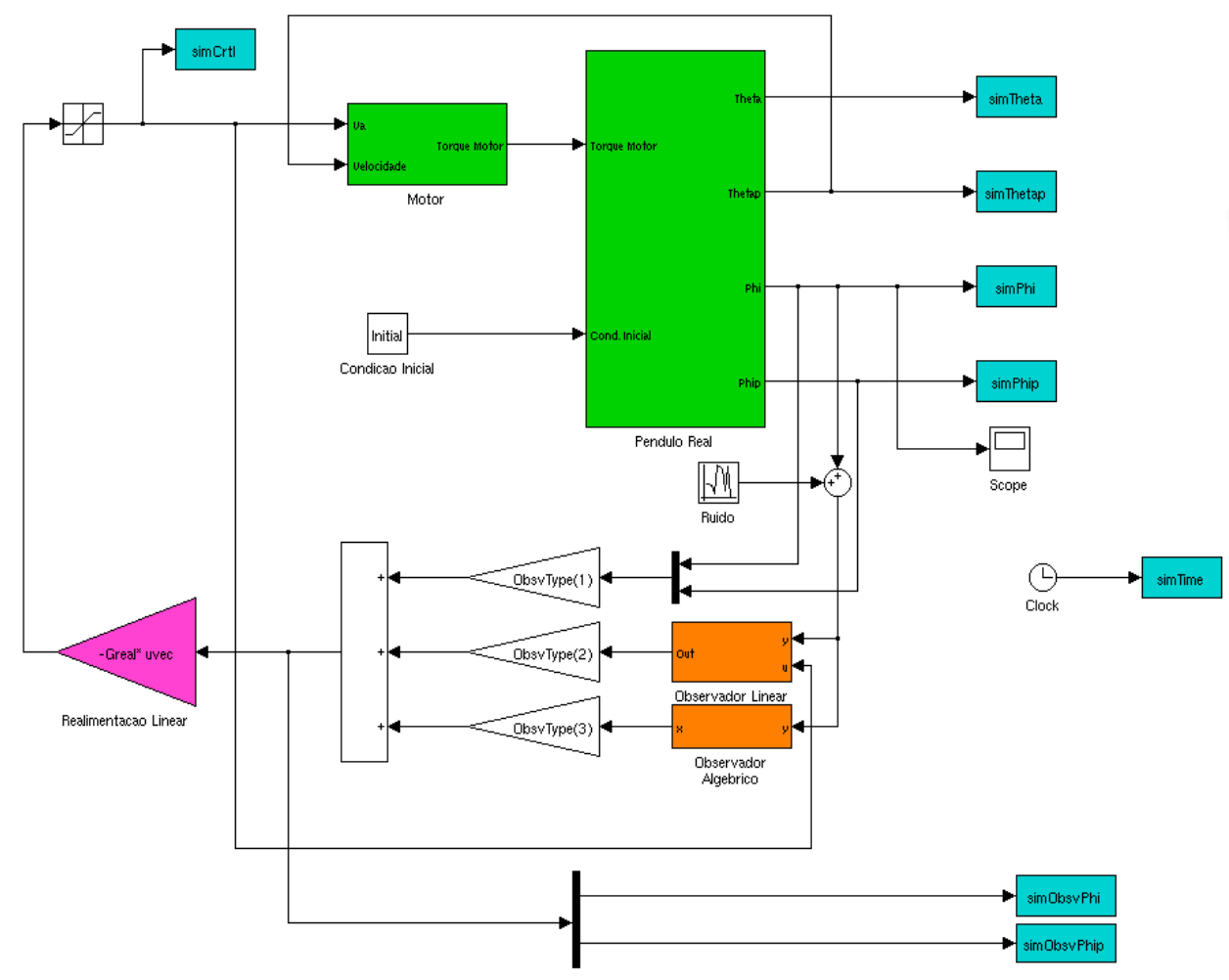

Figura C.1: Modelo Global 


\section{C.3.2 Modelo completo do Pêndulo}

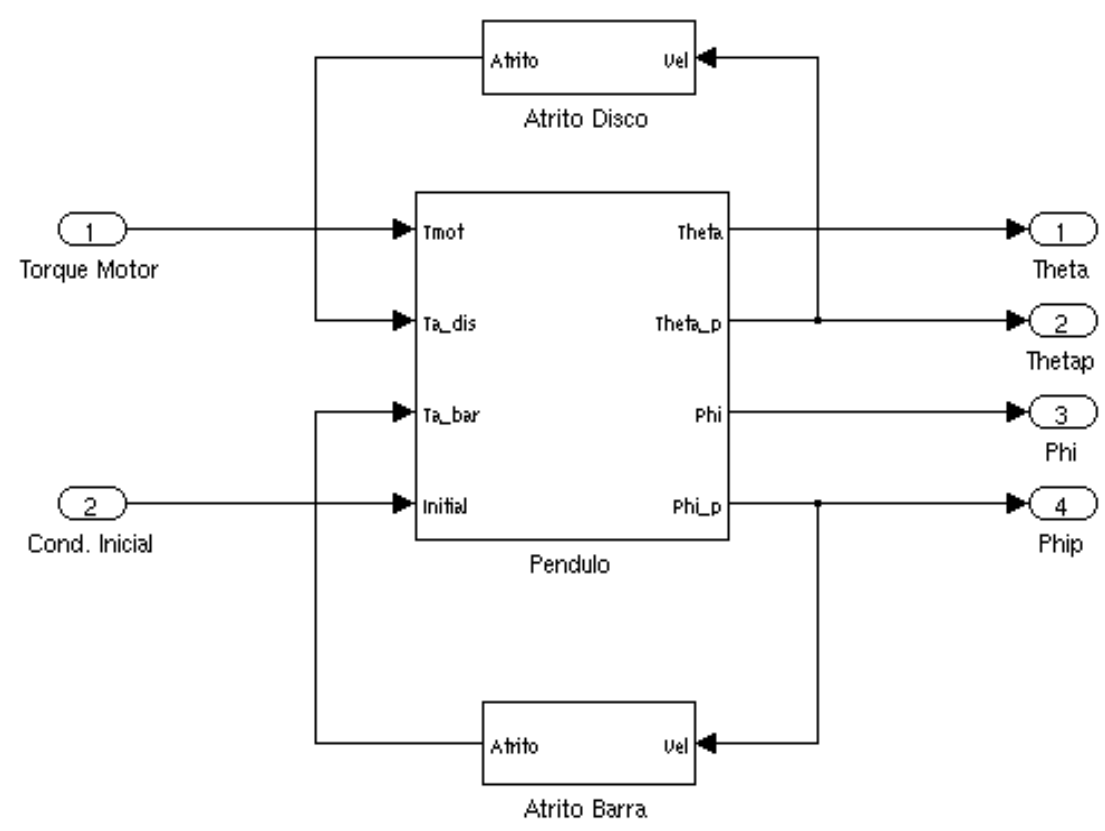

Figura C.2: Modelo Completo do Pêndulo.

\section{C.3.3 Modelo idealizado do Pêndulo}

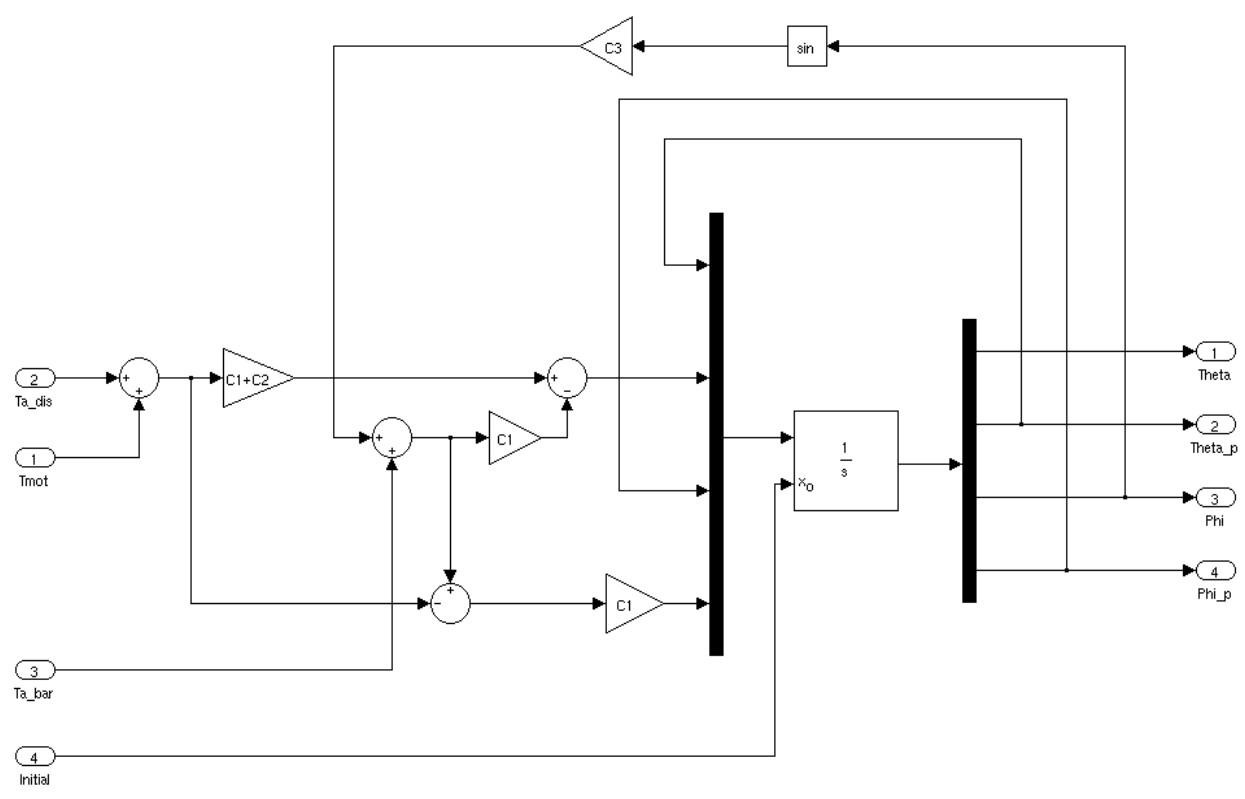

Figura C.3: Modelo Idealizado do Pêndulo. 


\section{C.3.4 Modelo do Motor}

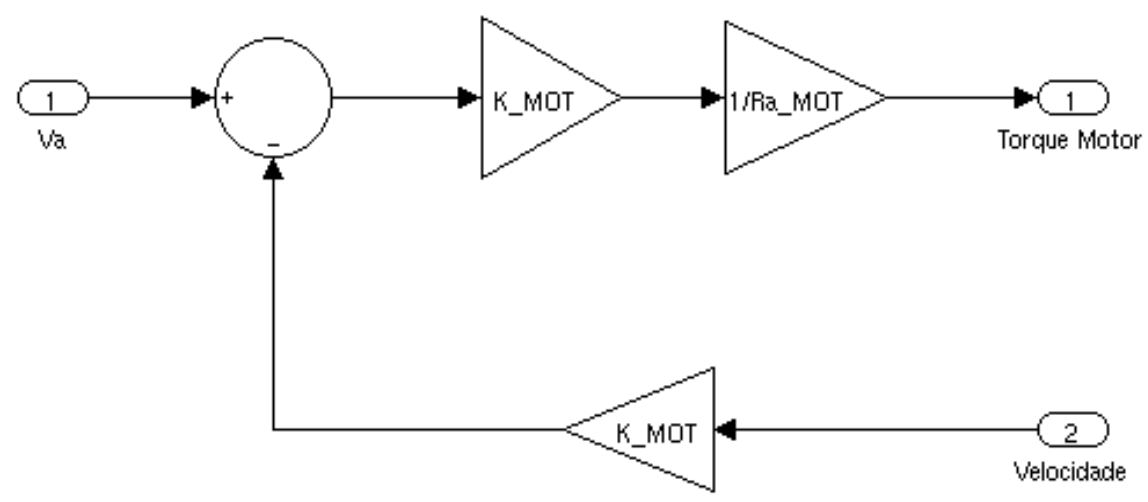

Figura C.4: Modelo do Motor.

\section{C.3.5 Modelo do Observador linear}

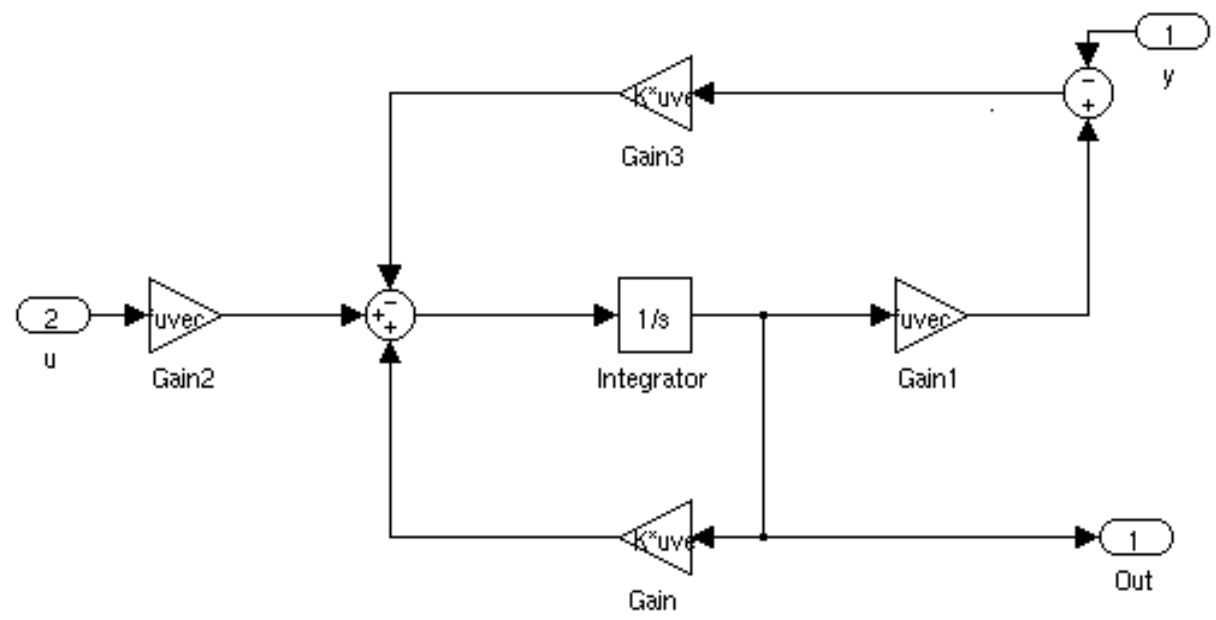

Figura C.5: Modelo do Observador Linear. 


\section{C.3.6 Modelo do Observador algébrico}

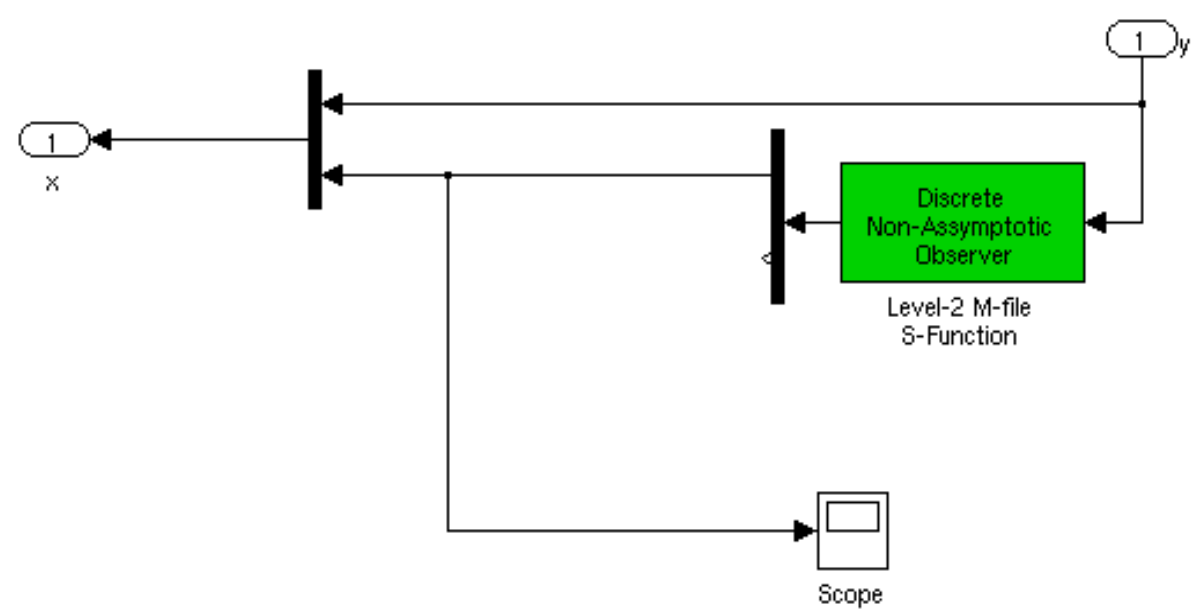

Figura C.6: Modelo do Observador Algébrico.

\section{C.3.7 Modelo gerador de atrito}

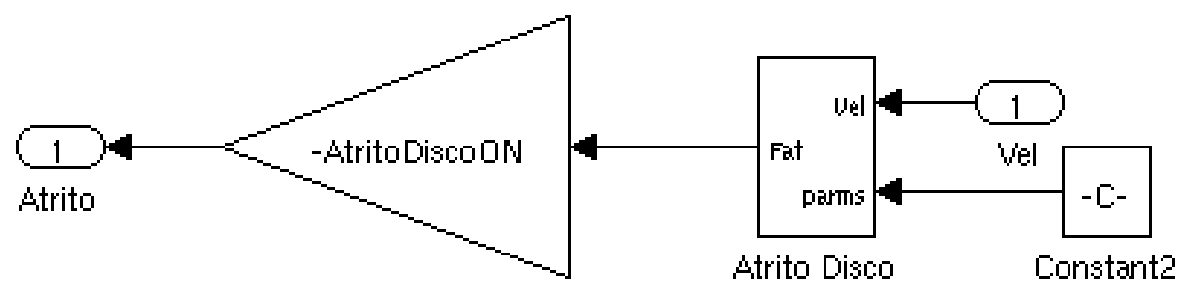

Gain2

Figura C.7: Modelo de Atrito.

\section{C.4 Programa para microcontrolador PIC}

O programa a seguir traduz os pulsos recebidos pelo encoder em um código binário que será lido pela placa Lynx.

; listwp $=18 \mathrm{f} 877 \mathrm{~A}$

\#include $<$ P16F877A.INC $>$

;******

;wConfig

_CONFIG_CP_OFFw\&_PWRTE_ON \&_WDT_OFF \&_LVP_OFF \&_XT_OSC 
;wVariaveis

CountL equ 0x020

CountH equ 0x021

Input 1 equ 0x022

Input 2 equ $0 \mathrm{x} 023$

Sample equ 0x024

;******

;wreset vector

org 00000h

goto Start

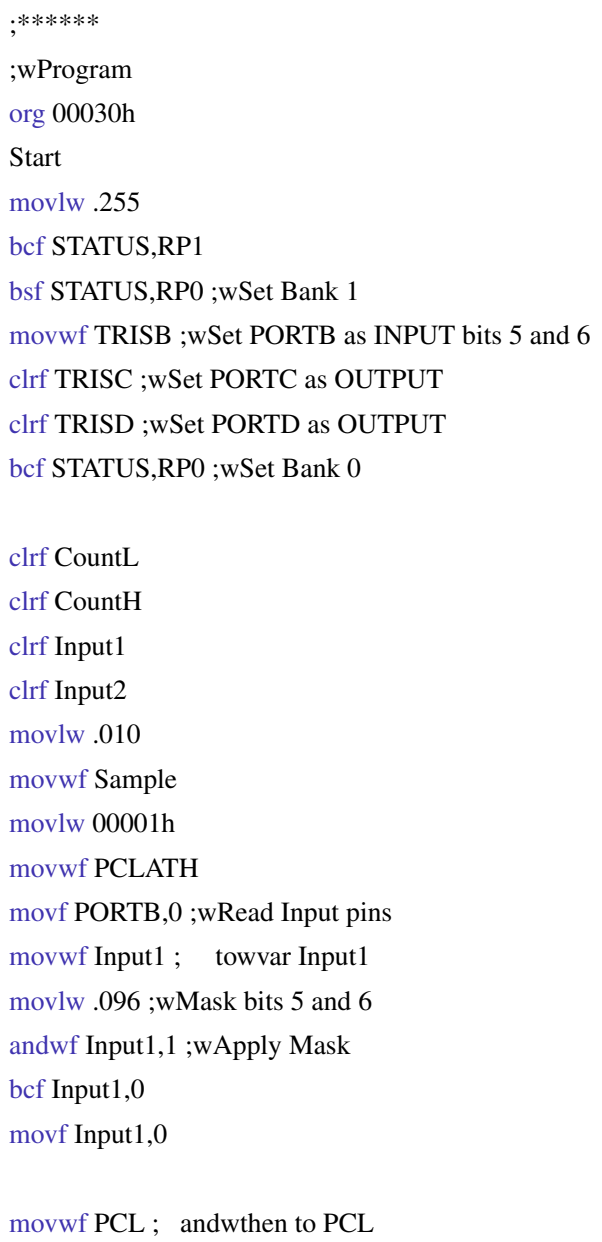


btfsc STATUS,Z ; wif Carry

incf CountH, 1 ; increment CountH

CheckLoop0_p1

btfss Input 1,6 ; ifwtoggle bit 6, decrement

goto RedoLoop

decf CountL, 1

comf CountL, 0

btfsc STATUS,Z ; wif Carry

decf CountH, 1 ;w decrement CountH

goto RedoLoop

org $00120 \mathrm{~h}$

CheckLoop1

; Wewknow channel inputs were 0 and 1

btfss Input1,5; ifwtoggle bit 5, increment

goto CheckLoop1_p1

incf CountL, 1

btfsc STATUS,Z ; wif Carry

incf CountH, 1 ;w increment CountH

CheckLoop1_p1

btfsc Input 1,6 ; ifwtoggle bit 6, decrement

goto RedoLoop

$\operatorname{decf}$ CountL, 1

comf CountL, 0

btfsc STATUS,Z ; wif Carry

decf CountH, 1 ;w decrement CountH

goto RedoLoop

org 00140h

CheckLoop2

; Wewknow channel inputs were 1 and 0

btfsc Input 1,5; ifwtoggle bit 5, increment

goto CheckLoop2_p1

incf CountL, 1

btfsc STATUS,Z ; wif Carry

incf CountH, 1 ;w increment CountH

CheckLoop2_p1

btfss Input 1, 6 ; ifwtoggle bit 6, decrement

goto RedoLoop

$\operatorname{decf}$ CountL, 1

comf CountL, 0

btfsc STATUS,Z ; wif Carry

decf CountH, 1 ;w decrement CountH

goto RedoLoop

org $00160 \mathrm{~h}$

CheckLoop3

; Wewknow channel inputs were 1 and 1

btfsc Input 1,5 ; ifwtoggle bit 5 , increment goto CheckLoop3_p1

incf CountL, 1 
btfsc STATUS,Z ; wif Carry

incf CountH, 1 ; increment CountH

CheckLoop3_p1

btfsc Input1,6 ; ifwtoggle bit 6, decrement

goto RedoLoop

decf CountL, 1

comf CountL, 0

btfsc STATUS,Z ; wif Carry

decf CountH, 1 ;w decrement CountH

RedoLoop

btfsc PORTB, 0 ;wSample, if clear, load Sample

gotow UpdateOutput

movlw .010

movwf Sample ;wLoad a count value in Sample

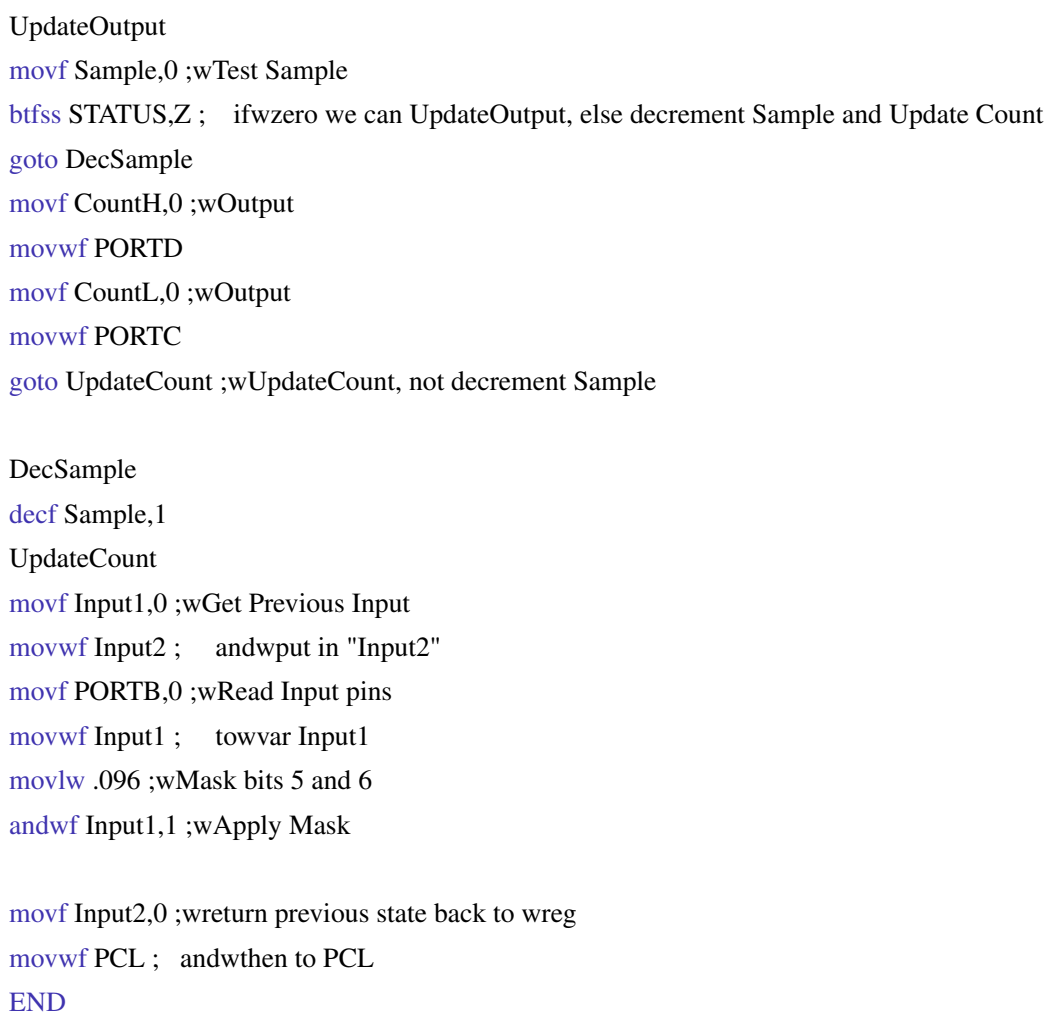




\section{C.5 Diagrama elétrico dos dispositivos de interface}

\section{C.5.1 Circuito de leitura do encoder}

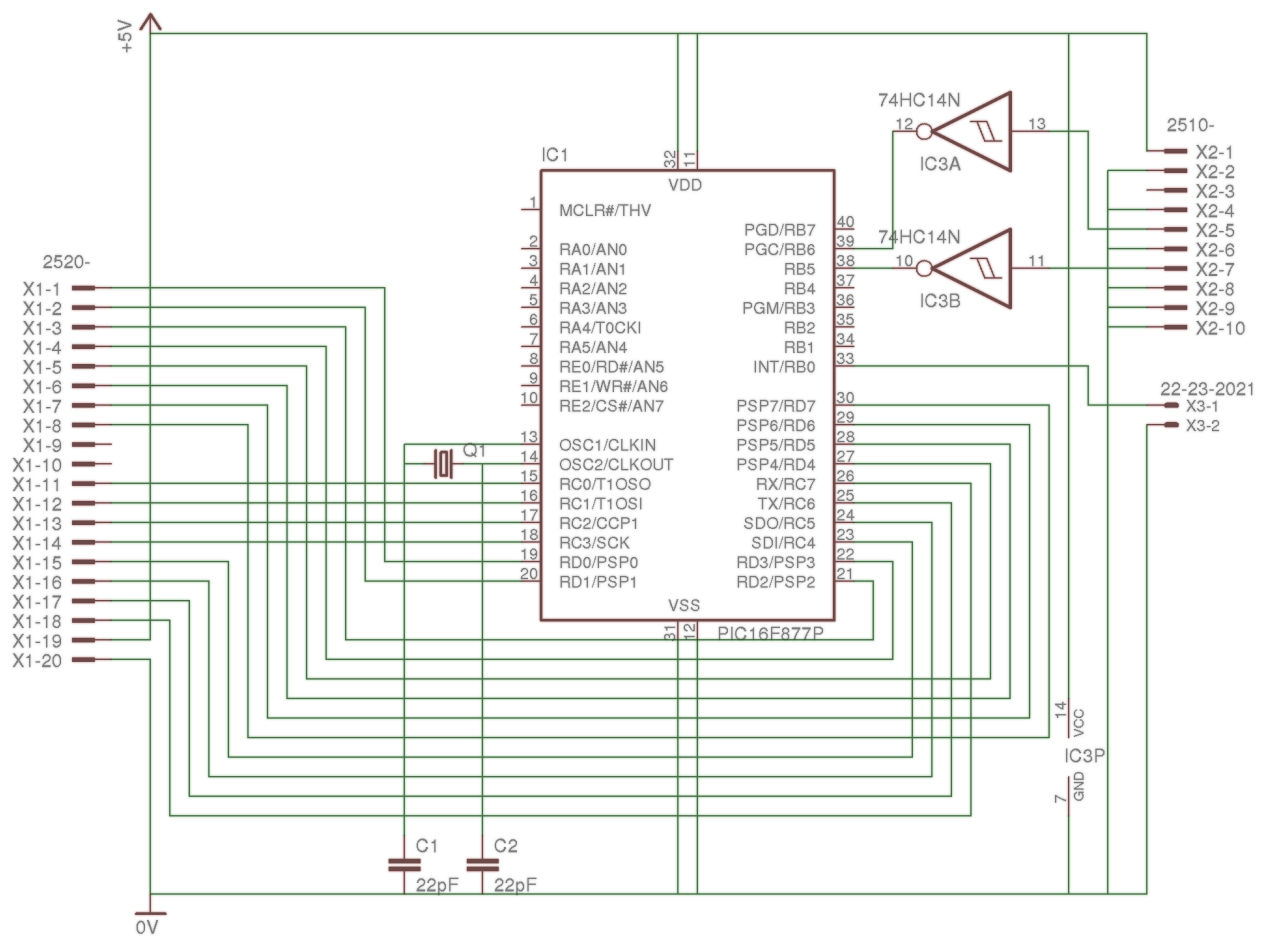

Figura C.8: Circuito elétrico do leitor do encoder 


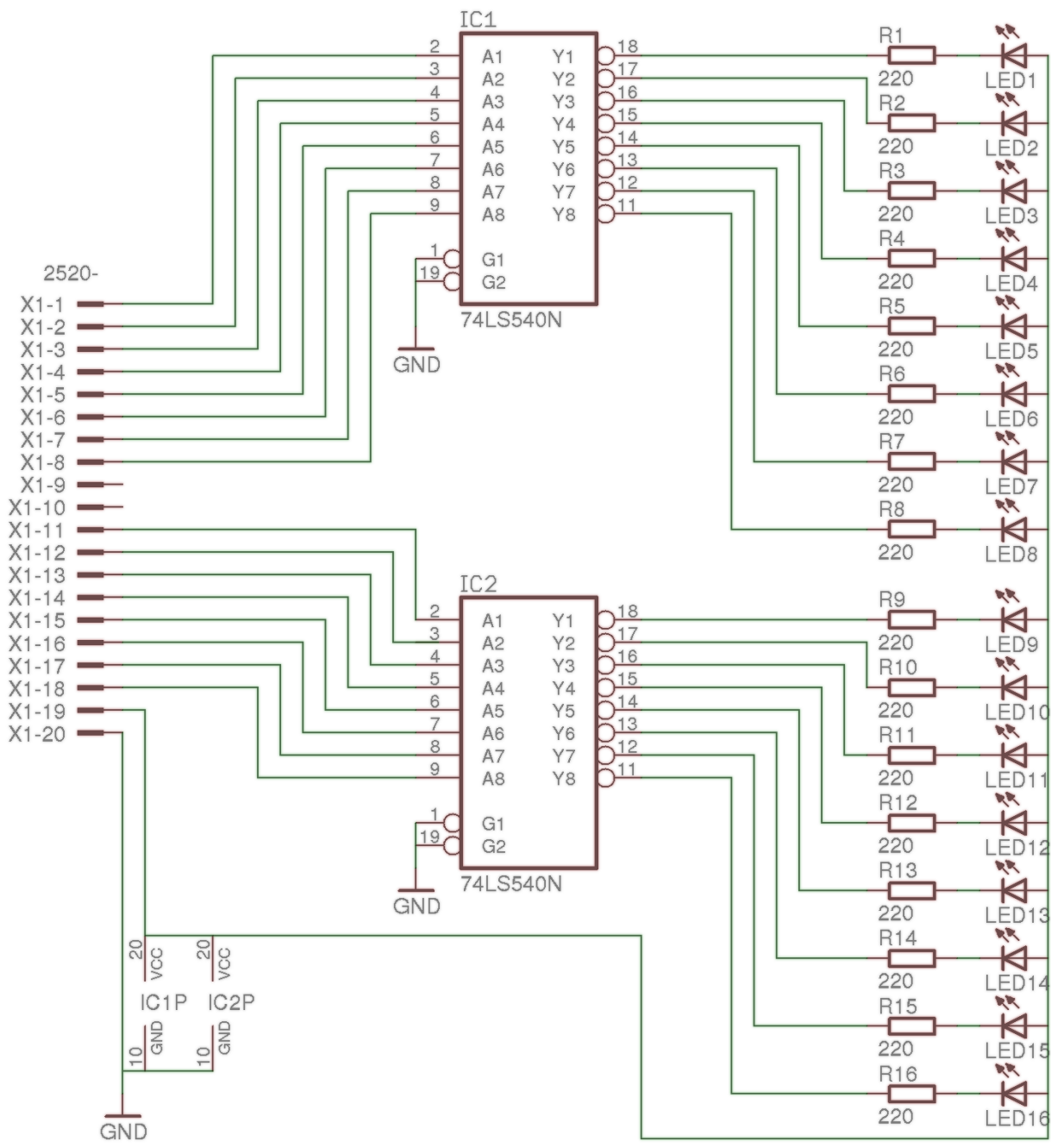

Figura C.9: Circuito elétrico do display de leds 


\section{C.5.2 Circuito de saída de potência}

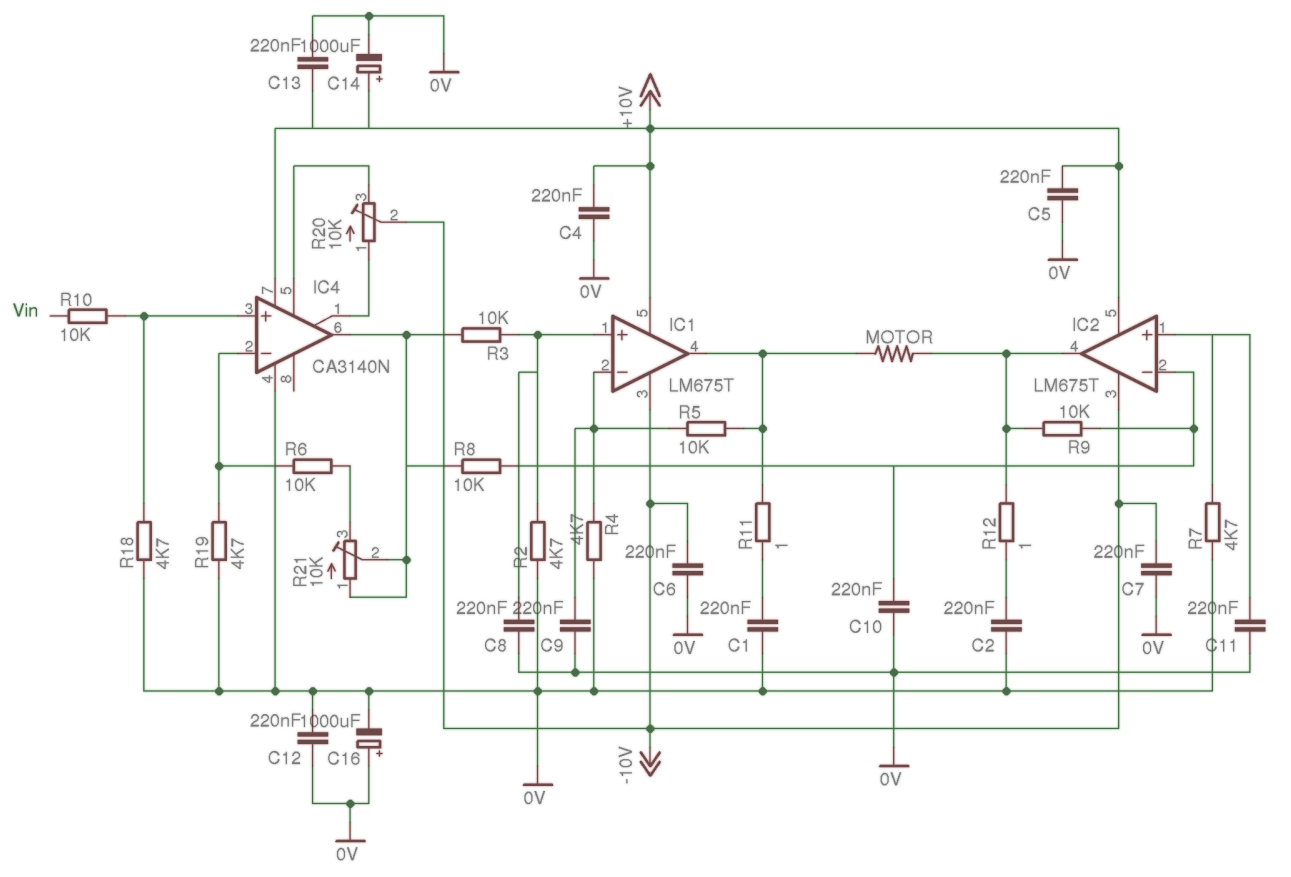

Figura C.10: Circuito elétrico do estágio de potência 


\section{C.6 Desenho das peças usinadas na construção do pêndulo}

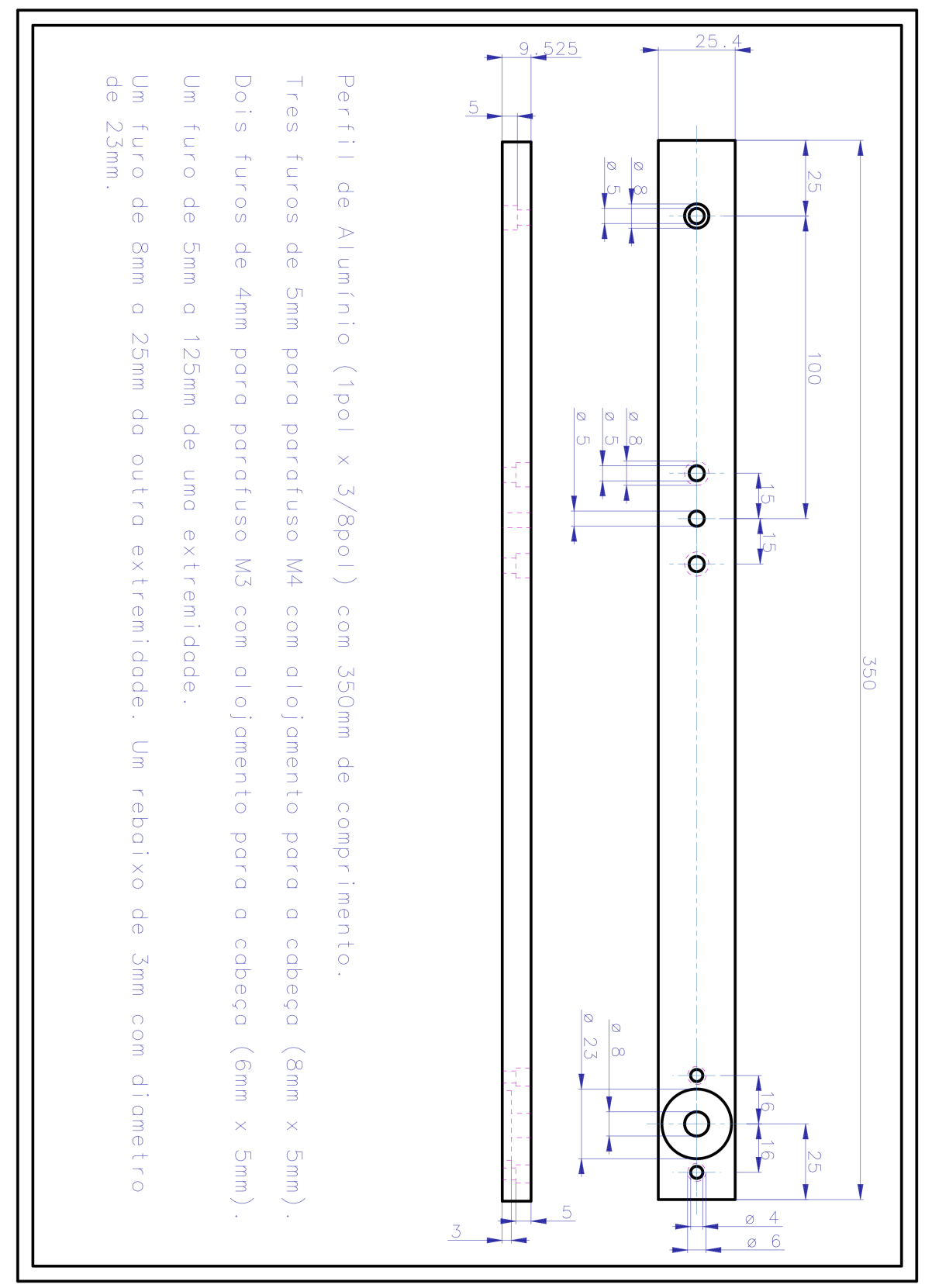

Figura C.11: Desenho da Barra 


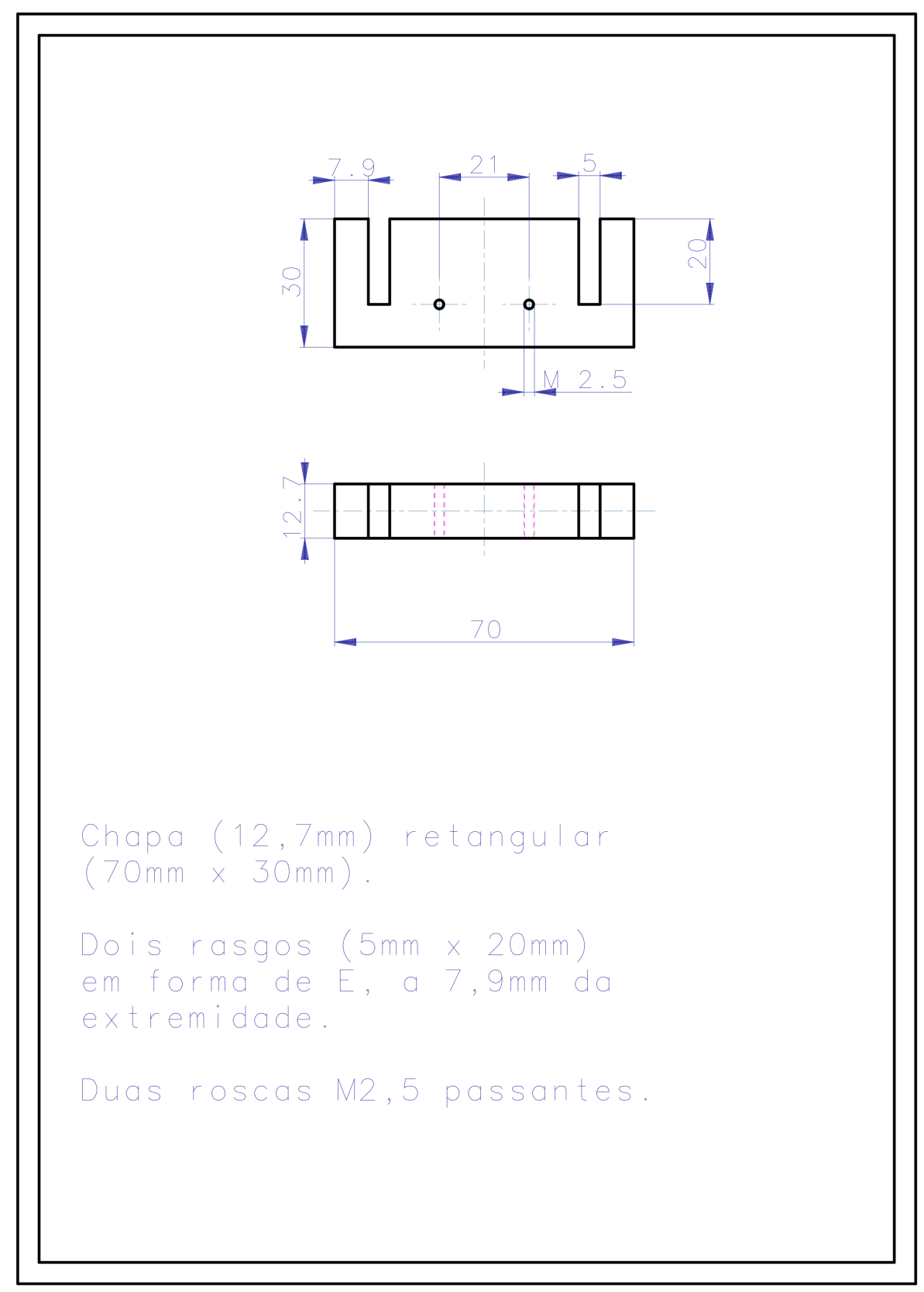

Figura C.12: Chapa de Fixação do Encoder. 


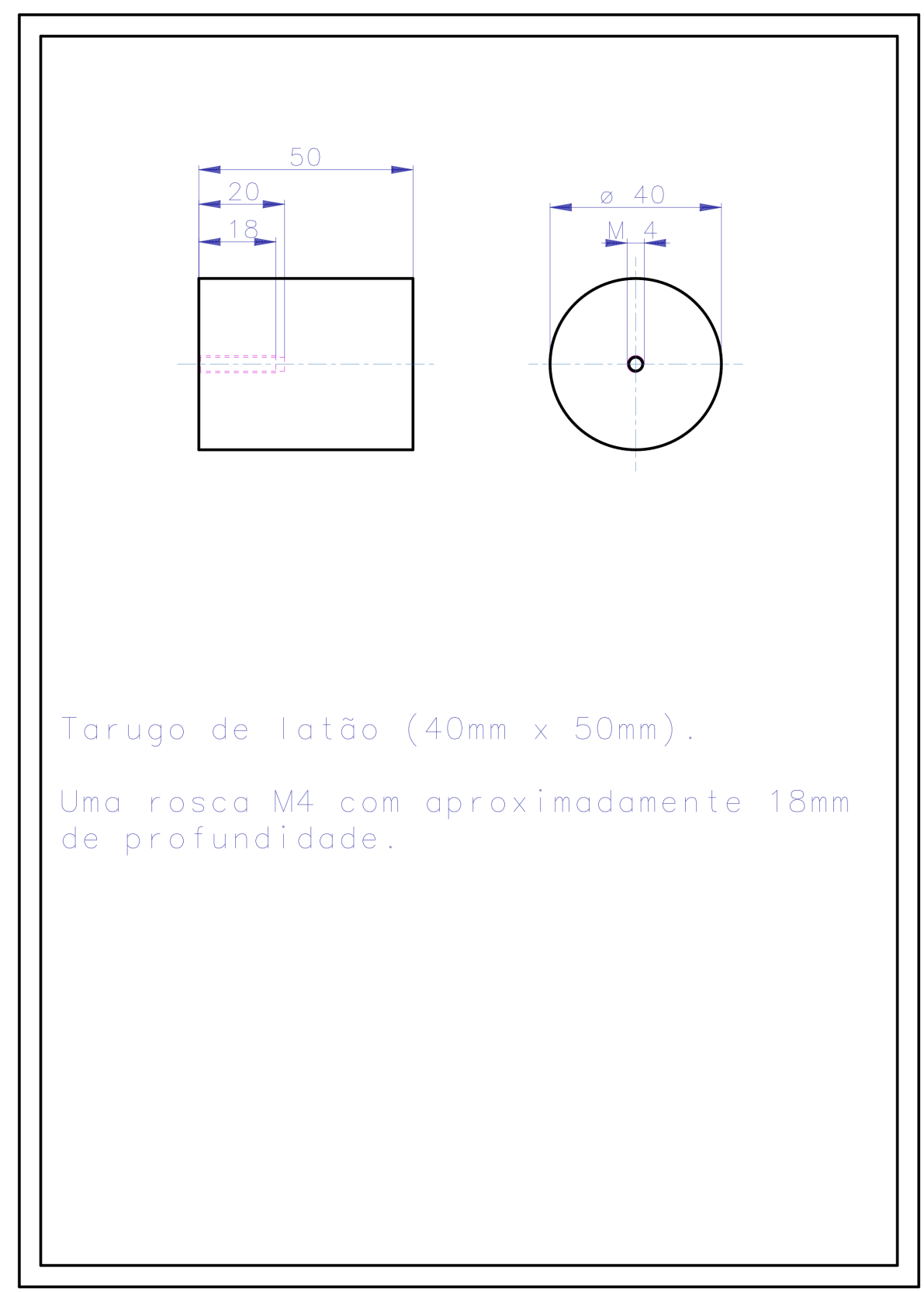

Figura C.13: Contra Peso. 

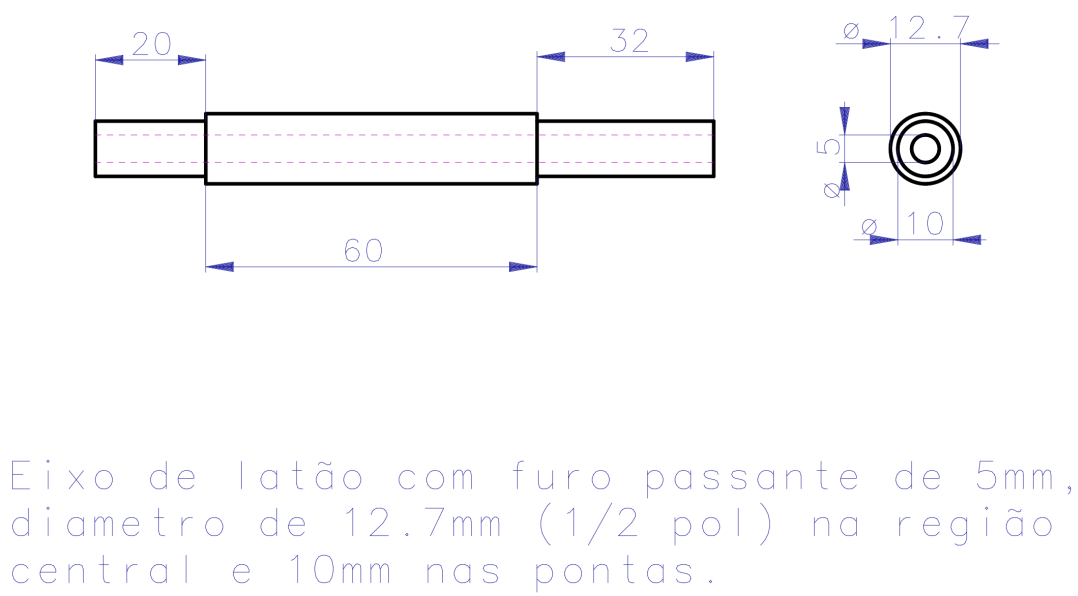

Figura C.14: Eixo. 


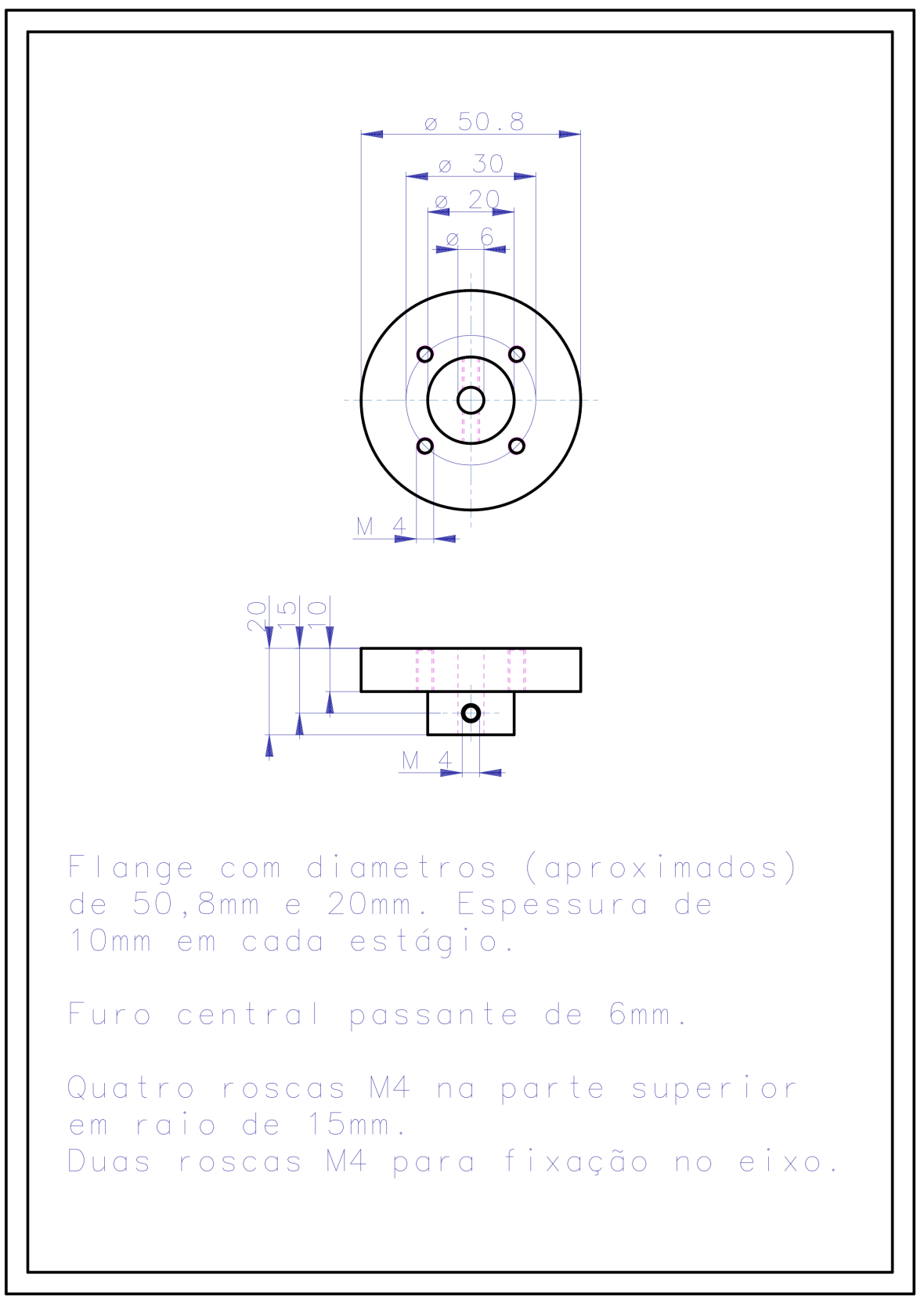

Figura C.15: Flange do Disco. 


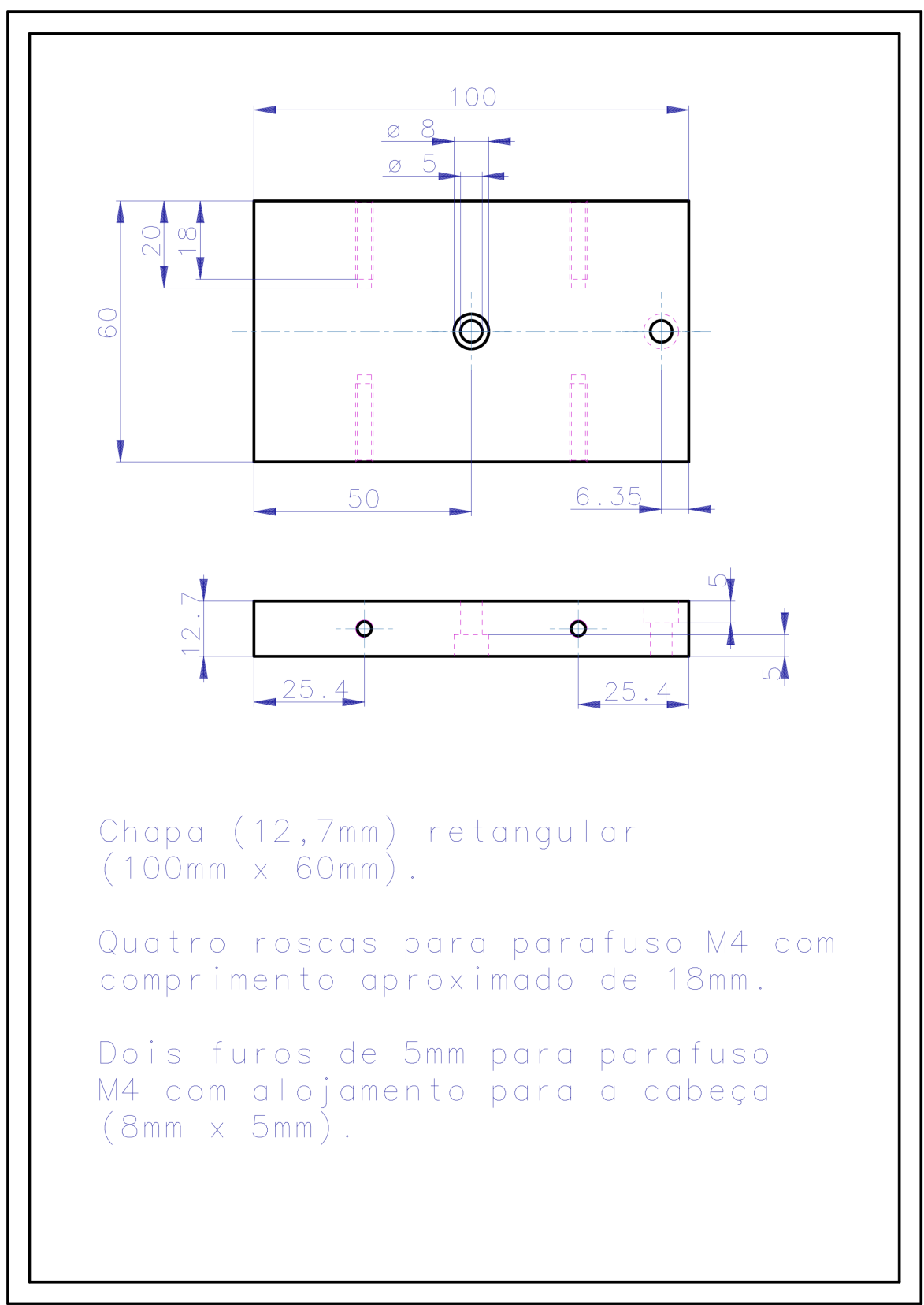

Figura C.16: Lateral 1. 


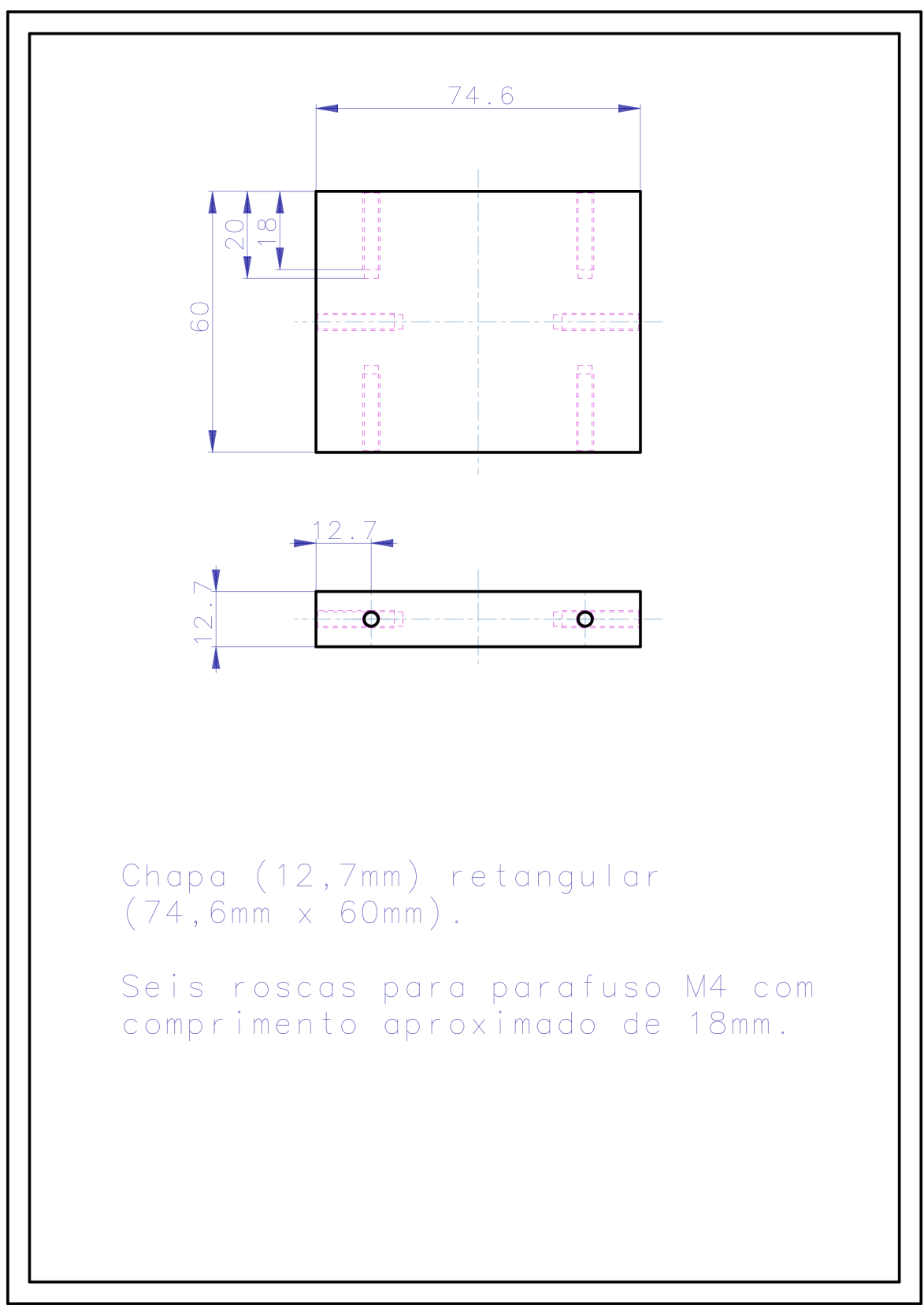

Figura C.17: Lateral 2. 


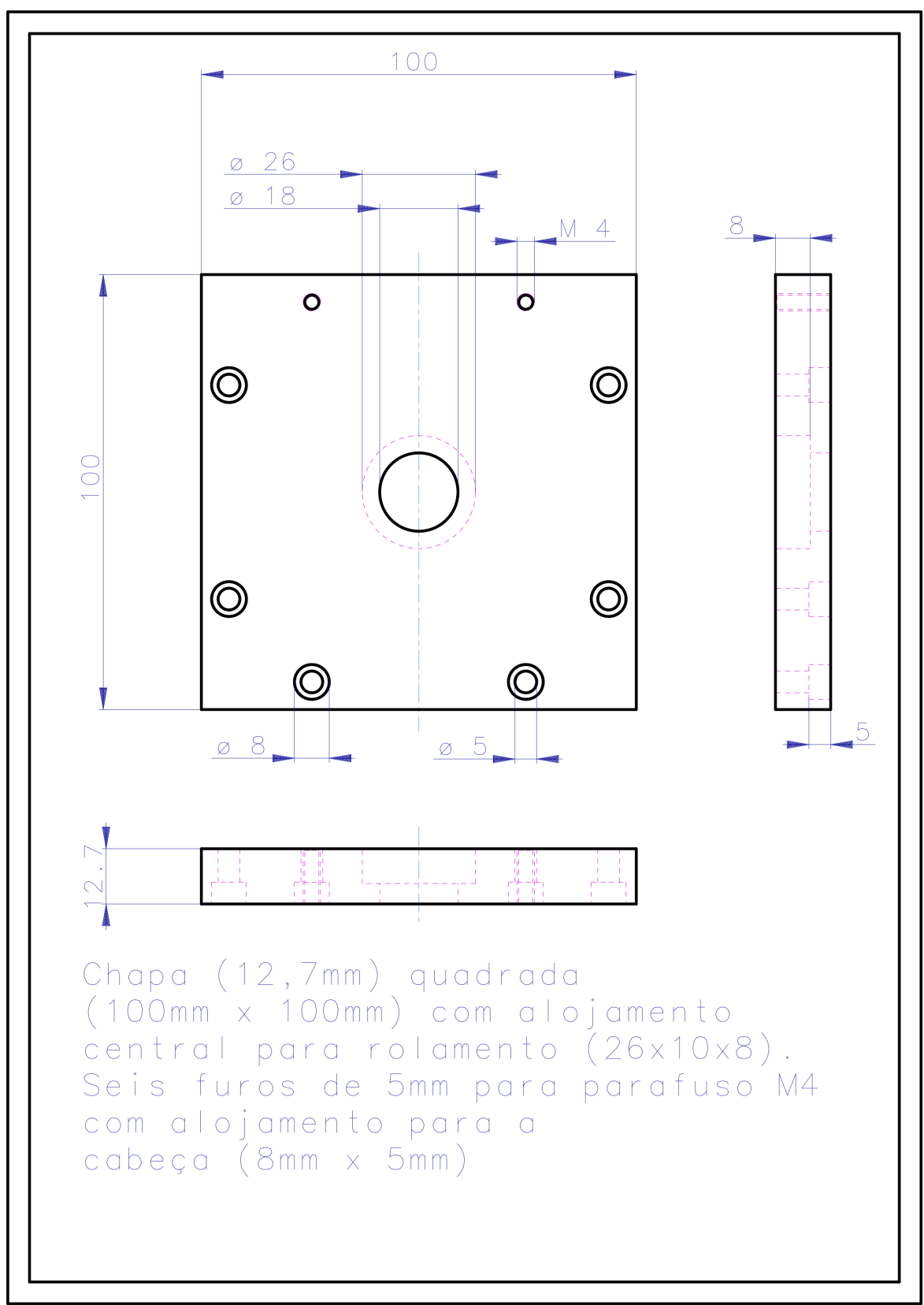

Figura C.18: Tampas. 


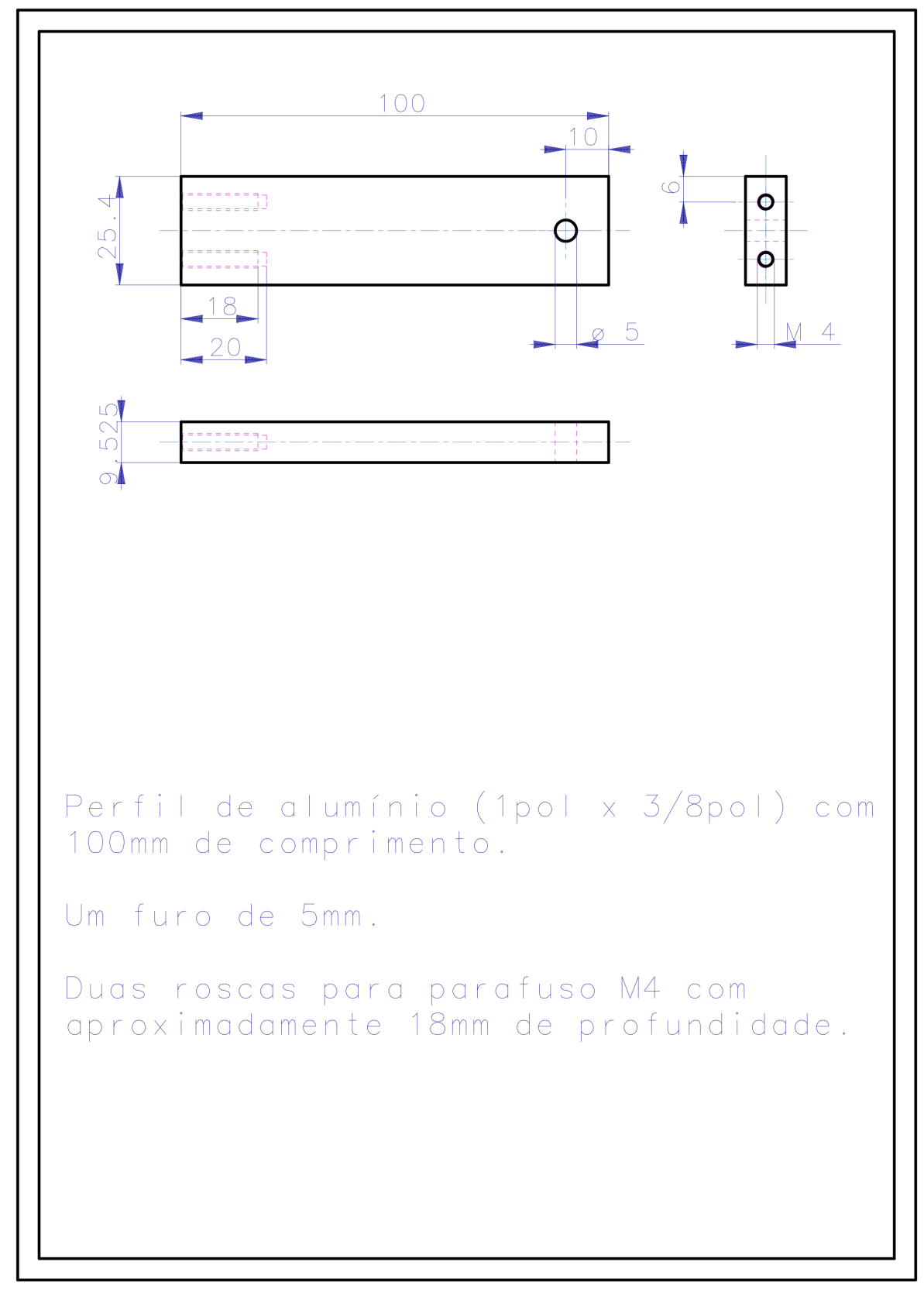

Figura C.19: Pilar. 


\section{C.7 Programas em linguagem $\mathrm{C} / \mathrm{C}++$}

\section{C.7.1 Interface para o subsistema Xenomai}

Xenomai é uma extensão para a kernel do sistema operacional linux, capaz de realizar o tratamento de rotinas e periféricos em tempo real. Os arquivos a seguir listam o código necessário para disponibilizar as funções da placa de aquisição e controle LYNX, e do seu módulo (driver) escrito utilizando a extensão Xenomai. Desta maneira foi possível escrever programas em linguagem $\mathrm{C}$ e $\mathrm{C}++$, em um sistema baseado em PC mas dedicado para controle.

\section{Arquivo LynxClass.h}

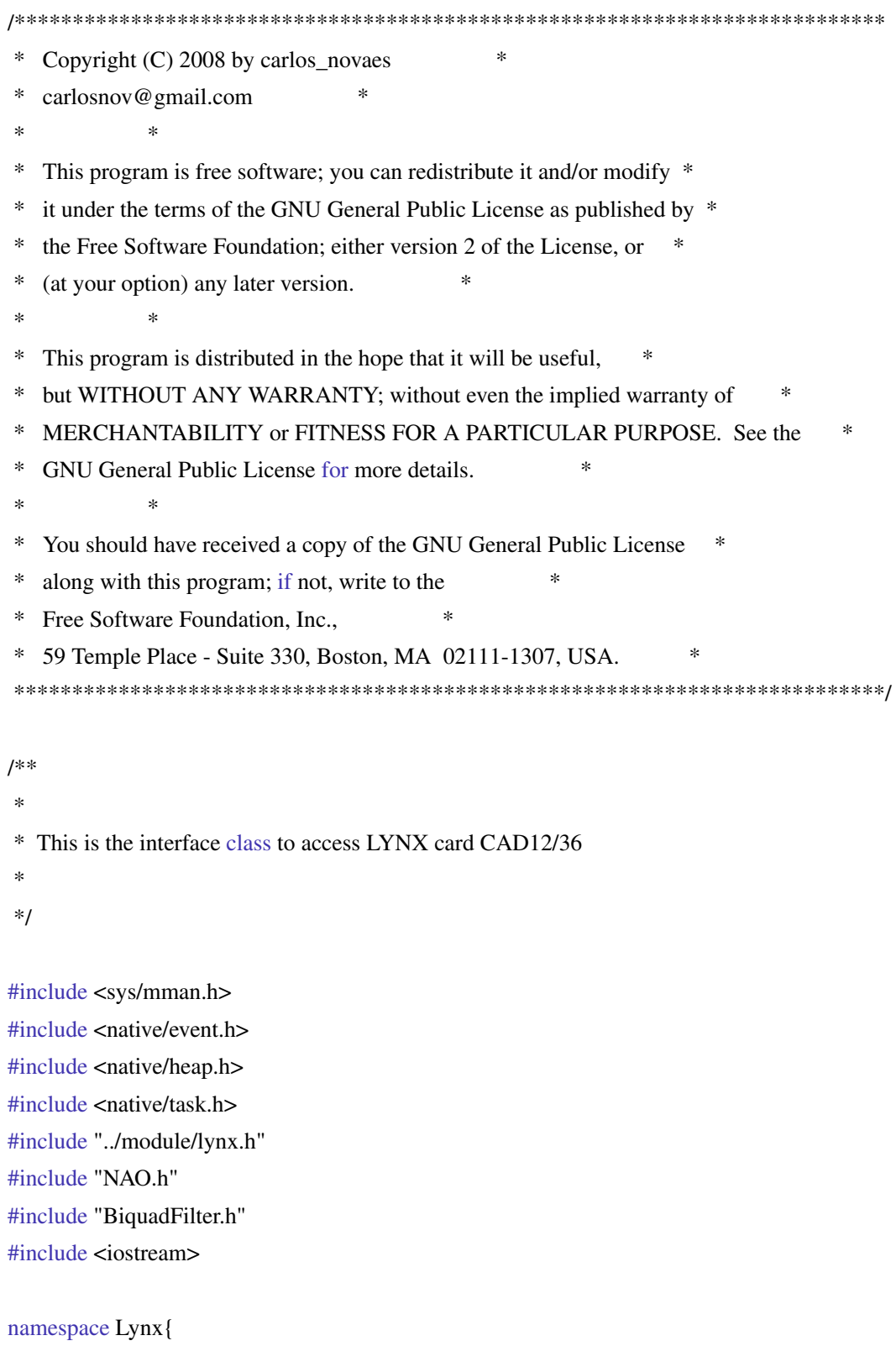




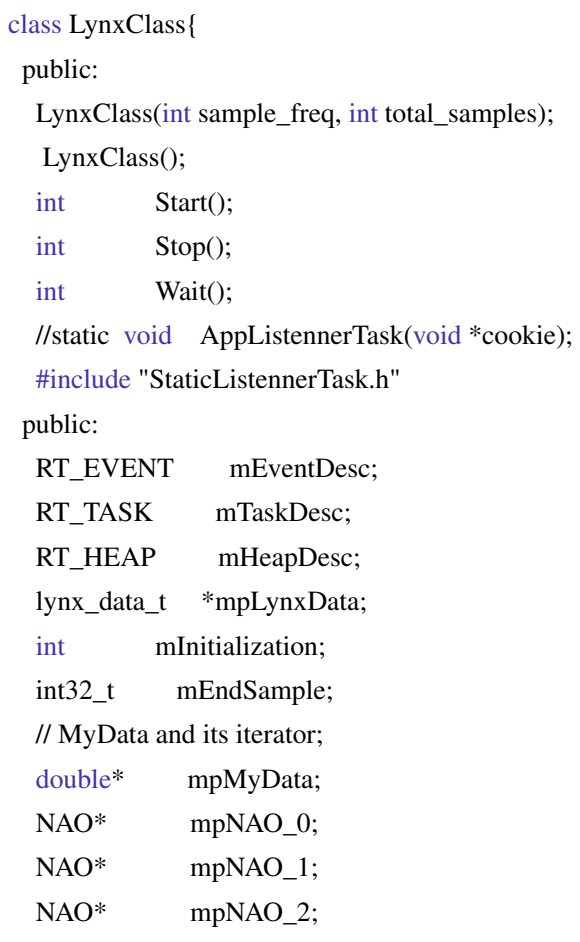

BiquadFilter* mpBiquadFilter_0;

\};

\};

Lynx::LynxClass::LynxClass(int sample_freq, int total_samples)\{

int result;

// Initialization flag;

mInitialization $=0$;

\#include "LynxClassInitialization.h"

// try to lock memory

result = mlockall(MCL_CURRENTIMCL_FUTURE);

if (result !=0)\{

std::cerr « "Cant lock memory«< std::endl;

mInitialization-;

\}

// try to bind Heap

result $=$ rt_heap_bind $(\& m H e a p D e s c$, LYNX_HEAP_NAME, TM_NONBLOCK);

if (result) \{

std::cerr « "Cant bind to Heap«< std::endl;

mInitialization-;

\}

// try to allocate Heap on this process

result $=$ rt_heap_alloc $(\& m H e a p D e s c, 0$, TM_NONBLOCK, $($ void**)\&mpLynxData);

if (result) \{

std::cerr « "Cant map memory heap«< std::endl;

mInitialization-; 


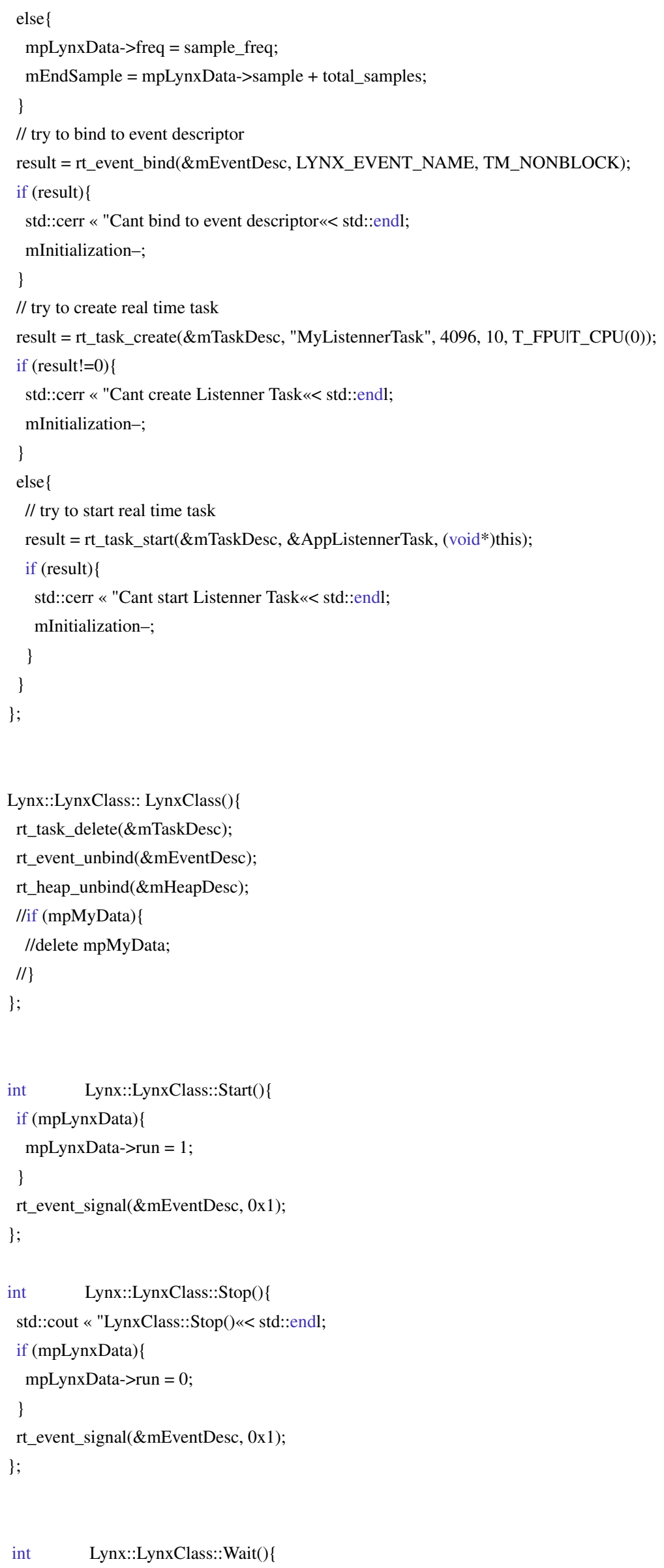




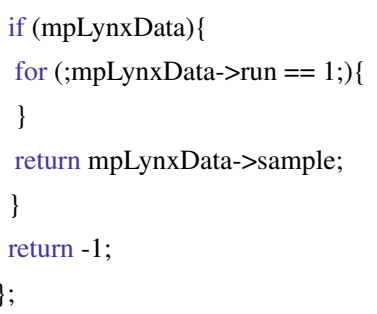

// End Of File 


\section{Arquivo LynxClassTest.cpp}

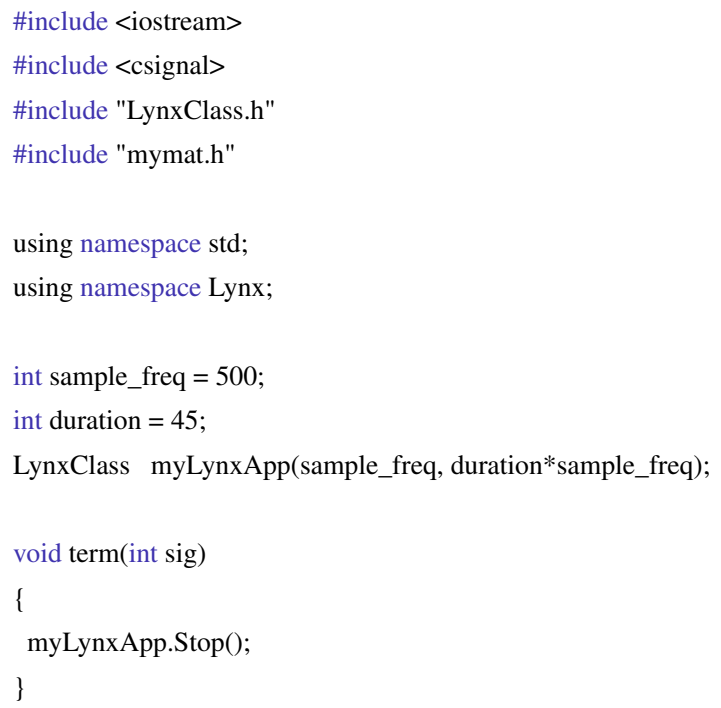




\section{Arquivo LynxClassInitialization.h}

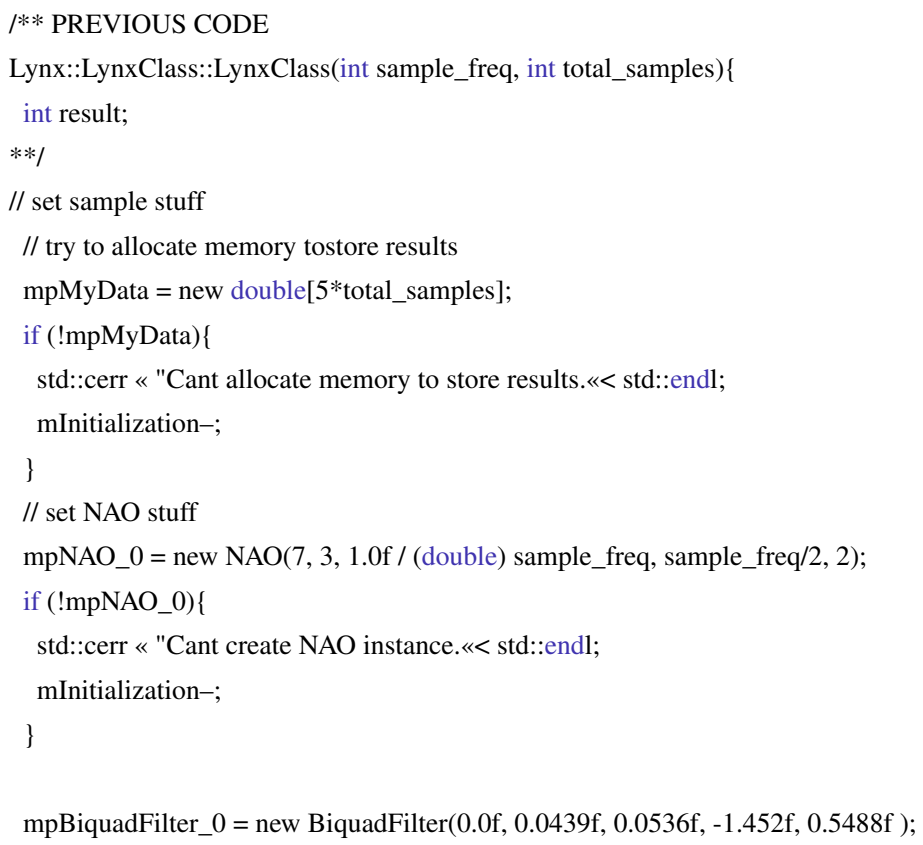




\section{Arquivo StaticListennerTask.h}

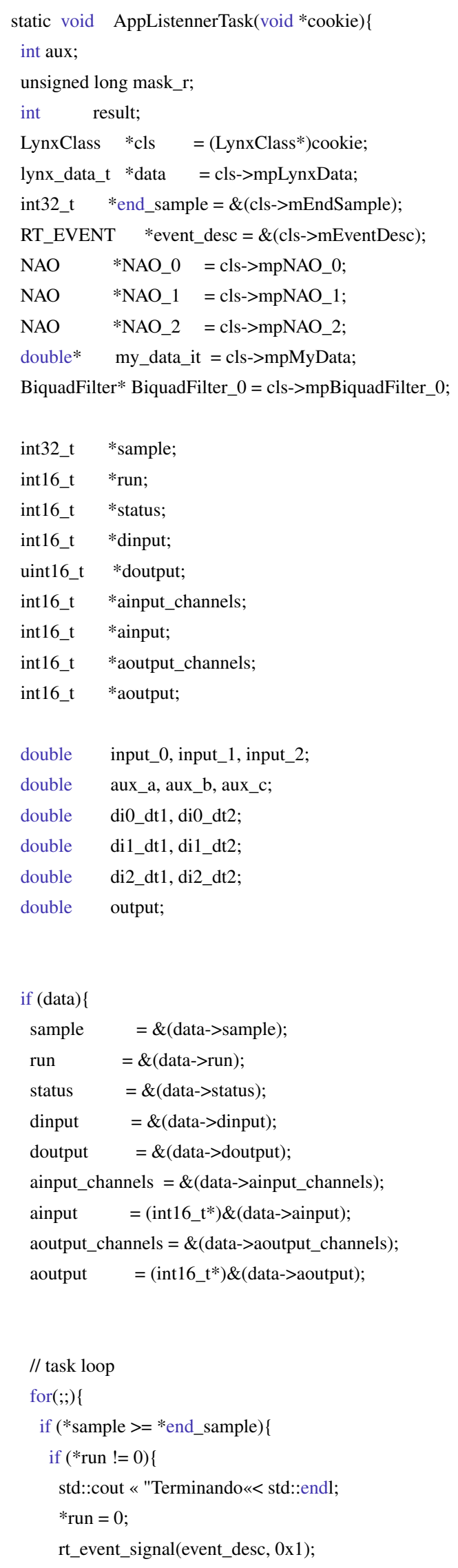




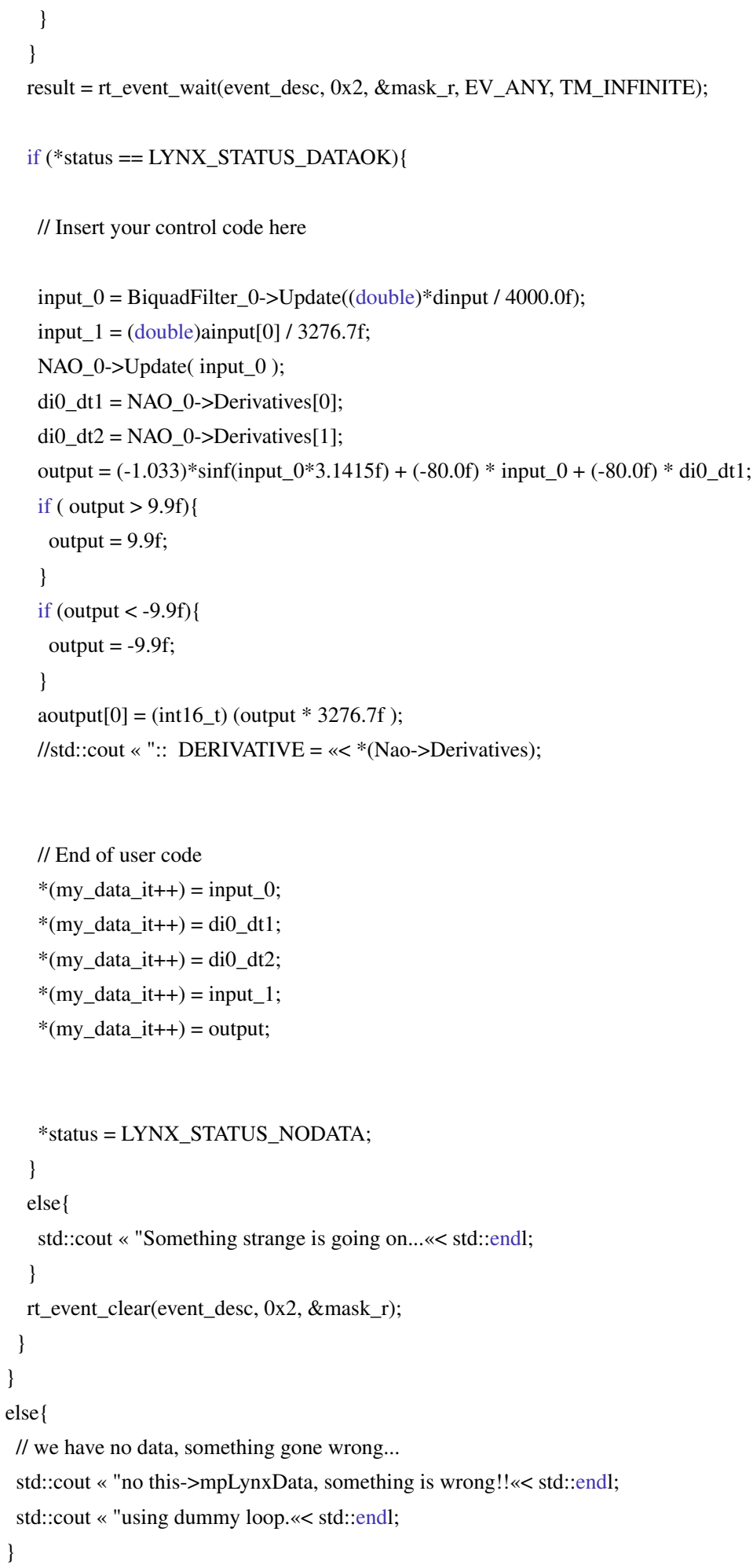




\section{C.7.2 Implementação discreta do observador algébrico}

Os arquivos a seguir listam o código necessário para implementar a teoria de estimadores rápidos, segundo a metodologia descrita no capítulo 3.

\section{Arquivo NAO.h}

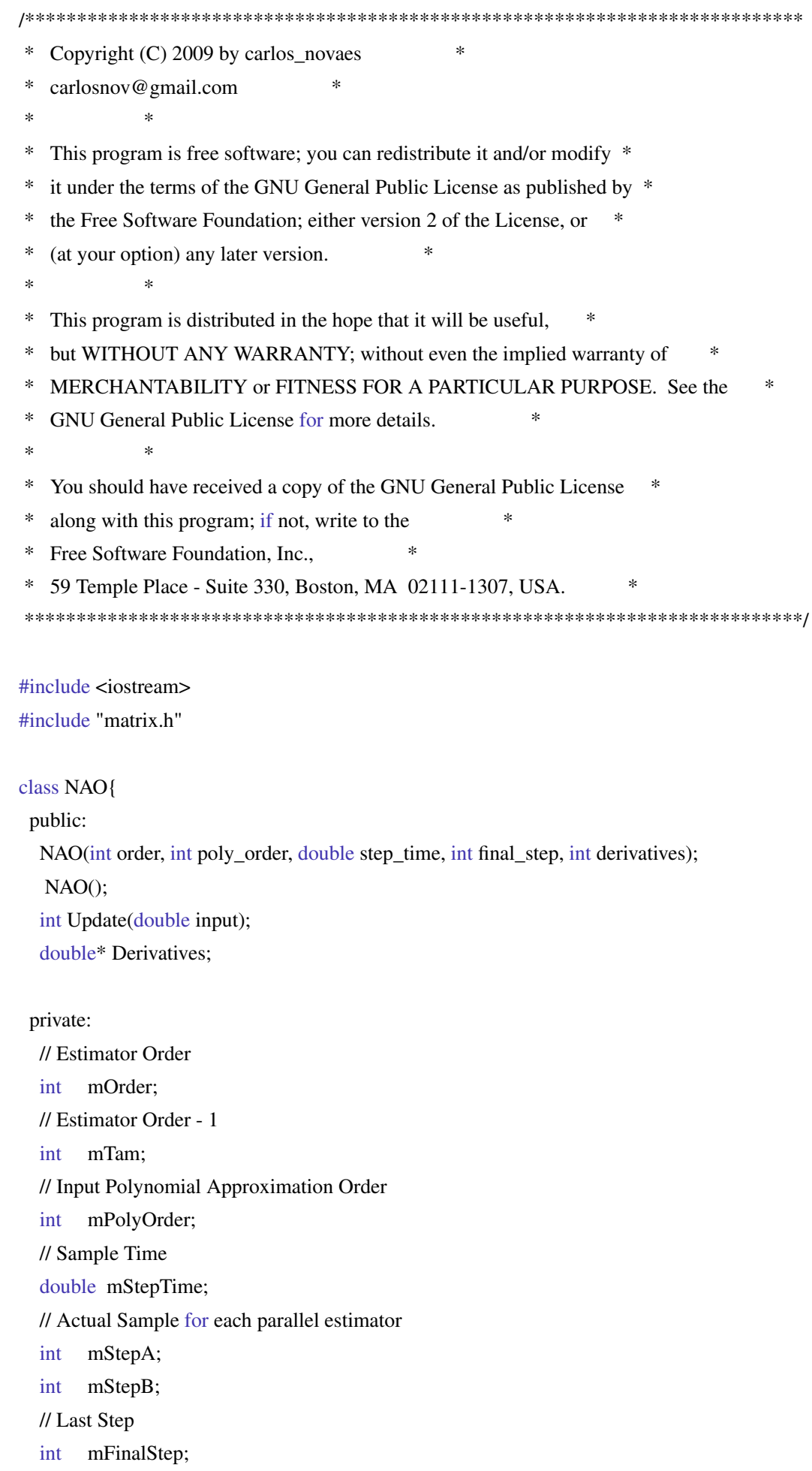


int mResetTime;

// how many derivatives we should calculate

int mMaxDerivatives;

// true if there is enough data points to PolyGets Matrix

bool mDataOk;

// from which estimator should we get derivatives

int mUseEstimator;

// Pointer to Start of Input Buffer

double* mpInputBuffer;

// Iterator for Input Buffer

double* mpInputBufferIt;

// Pointer to Start of PolyGets Matrix Coefficients

double* mpPolyGets;

// Iterator to PolyGets

double* mpPolyGetsIt;

// Polynomial Coefficients

double* mpPolyCoefs;

// Iterator to Polinomial Coefficients

double* mpPolyCoefsIt;

// CCoefs and its Iterator

double* mpCCoefs;

double* mpCCoefsIt;

// DCoefs and its Iterator

double* mpDCoefs;

double* mpDCoefsIt;

// ECoefs and Its Iterator

double* mpECoefs;

double* mpECoefsIt;

// TPowers and its Iterator

double* mpTPowers;

double* mpTPowersIt;

// Delta Time Powers Coefficients and its Iterator

double* mpDTCoefs;

double* mpDTCoefsIt;

// ZFilter, Tam for each estimator

double* mpZFilterA;

double* mpZFilterB;

// ZFilter Iterator

double* mpZFilterIt;

// ZDiff

double* mpZDiff;

// ZDiff Iterator

double mDiff1;

double* mpZDiffIt;

// Derivatives and its Iterator

double* mpDerivativesIt;

\}

// End Of File 


\section{Arquivo NAO.cpp}

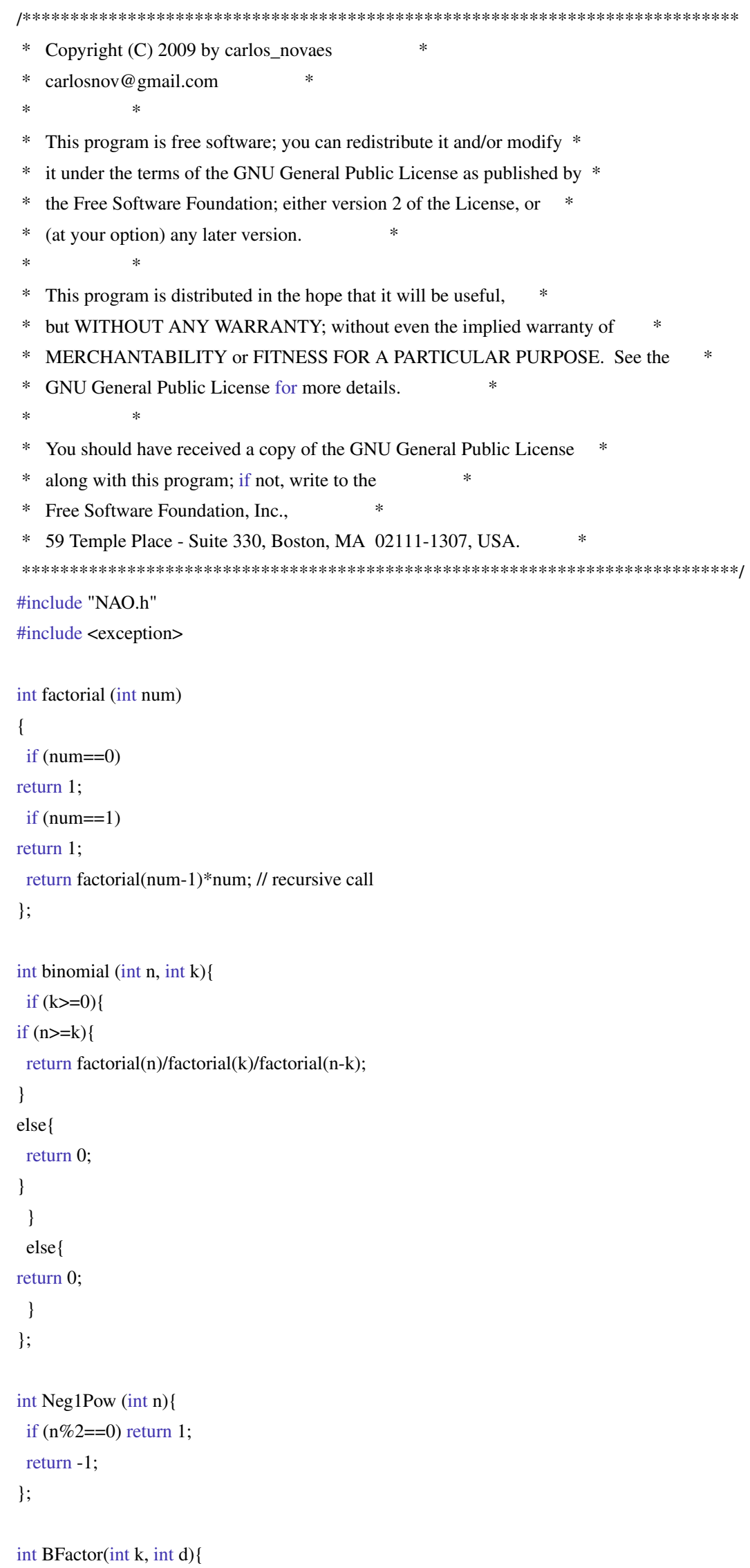




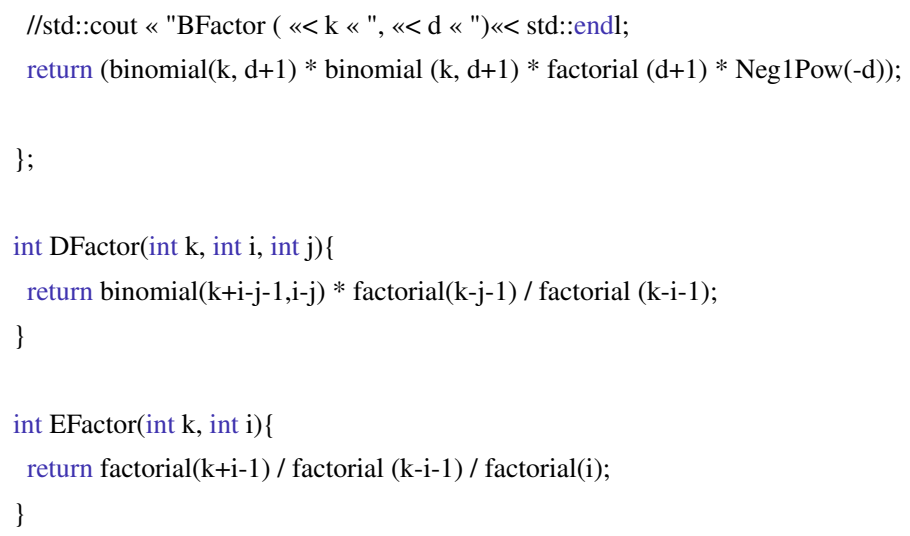

NAO::NAO(int order, int poly_order, double step_time, int final_step, int derivatives)\{ 
SLAP::Matrix MtzAlpha(poly_order+1);

for (it_i=0; it_i<=final_step; it_i++) \{

for (it_j=0; it_j<=poly_order; it_j++) \{

for (it_k=0; it_k<=poly_order; it_k++) \{

MtzAlpha._Element $\left(i t \_j, i t \_k\right)=\operatorname{powl}\left(\left(i t \_i+i t \_j-\text { poly_order }\right)^{*}\right.$ step_time, poly_order - it_k $)$;

\}

\}

MtzAlpha.Inverse();

$/ / \mathrm{mpPolyGetsIt}=$ mpPolyGets;

for (it_j=0; it_j<=poly_order; it_j++) \{

for (it_k=0; it_k<=poly_order; it_k++) \{

//std::cout « "Elemento ( $\ll$ it_j $\ll ", \ll<$ it_k $\ll ")=\ll<$ MtzAlpha._Element(it_j, it_k) «std::endl;

*mpPolyGetsIt++ = MtzAlpha._Element(it_j, it_k);

\}

//MtzAlpha.Display();

//SLAP::Matrix* mtz = new SLAP::Matrix(MtzAlpha);

//mtz->Multiply(MtzBeta);

$/ / \mathrm{mtz}->$ Display();

//delete mtz;

\}

mpPolyGetsIt = mpPolyGets;

count_a $=0$;

for (it_l=1; it_l<=order-1; it_l++) \{

for (it_h $=1$; it_h $<=$ order-it_l; it_h++) \{

for (it_g=0; it_g<=poly_order; it_g++)\{

for (it_j=0; it_j<=order+it_g-it_h-it_l; it_j++)\{

count_a++;

\}

\}

\}

//std::cout « "CCoefs size is «< count_a « std::endl;

mpCCoefs = new double[count_a];

mpCCoefsIt $=$ mpCCoefs;

for (it_l $1=1$; it_l<=order- 1 ; it_l++) \{

for (it_h=1; it_h<=order-it_l; it_h++) \{

for (it_g=0; it_g<=poly_order; it_g++)\{

for (it_j=0; it_j<=order+it_g-it_h-it_l; it_j++)\{

*mpCCoefsIt = BFactor(order, it_1-1+it_h) * factorial(order+it_g-it_h-it_l) / factorial(it_j) / factorial(order+it_g-it_l-it_j) * powl(step_time, order+it_g-it_1-it_j);

$/ /$ printf(" $[1, \mathrm{~h}, \mathrm{~g}, \mathrm{j}]=[\% \mathrm{~d}, \% \mathrm{~d}, \% \mathrm{~d}, \% \mathrm{~d}]::$ CCoef=\%f\\{\}n", it_1, it_h, it_g, it_j, *mpCCoefsIt);

mpCCoefsIt++;

\}

\}

\}

mpCCoefsit $=$ mpCCoefs;

mpDCoefsit $=$ mpDCoefs; 


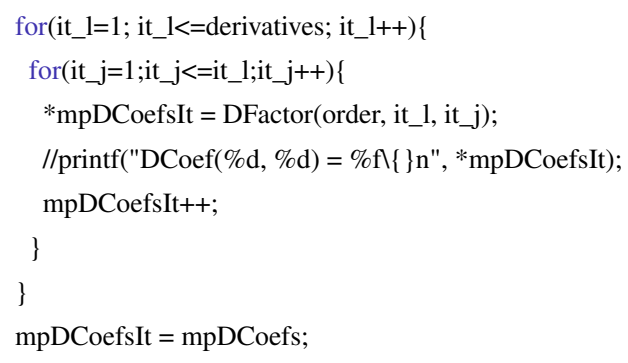

mpECoefsIt $=$ mpECoefs;

for(it_l=1; it_l<=derivatives; it_l++) \{

*mpECoefsIt $=$ EFactor(order, it_l 1 );

mpECoefsIt++;

\}

mpECoefsIt $=$ mpECoefs;

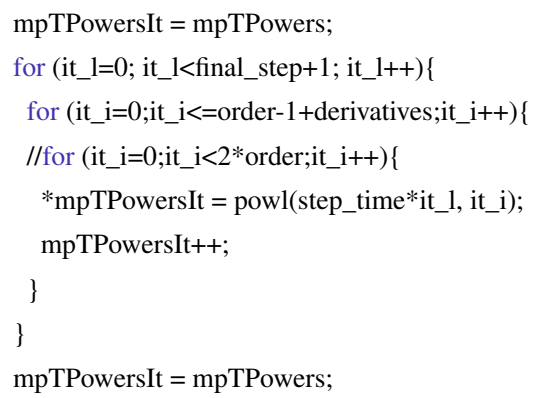

mpDTCoefsIt $=$ mpDTCoefs;

for (it_i $=0$;it_i<=order+poly_order,it_i++) \{

*mpDTCoefsIt $=$ powl $\left(\right.$ step_time, $($ double $\left.) i t \_i\right) /$ factorial(it_i $)$; mpDTCoefsIt++;

\}

mpDTCoefsIt $=$ mpDTCoefs;

mpZFilterIt $=$ mpZFilterA;

for (it_i $=0 ;$; $\left.\_i<o r d e r ; i t \_i++\right)$

$*($ mpZFilterIt++ $)=0.0 f$;

mpZFilterIt $=$ mpZFilterB;

for (it_i $\mathrm{i}=0 ; \mathrm{it} \_\mathrm{i}<$ order;it_i++)

$*(\mathrm{mpZFilterIt++})=0.0 \mathrm{f}$;

//std::cout «*(mpPolyGets+16) «std::endl; 
\}; NAO:: $\mathrm{NAO}()\{$

\}

int NAO::Update(double input)\{

int it_i, it_j, it_k, it_h, it_g, it_l;

double aux_a, aux_b, aux_c;

int count_a;

double *ptr_aux;

// Update Steps

mStepA++;

mStepB++;

// Fill in Input buffer

ptr_aux = mpInputBuffer;

mpInputBufferIt = mpInputBuffer;

for (it_i $=0$; it_i<mPolyOrder; it_i++) \{

*mpInputBufferIt++ = *++ptr_aux;

\}

*mpInputBufferIt $=$ input;

mpInputBufferIt $=$ mpInputBuffer;

//std::cout « "InputBuffer = [ ";

for (it_i $=0$; it_i<=mPolyOrder; it_i++)\{

//std::cout «mpInputBuffer[it_i] «", ";

\}

//std::cout «"]《<std::endl;

// Check if we already got enough data points

if ( $\mathrm{mDataOk}==$ false $)\{$

//std::cout « "Not OK!!!«<std::endl;

if $(\mathrm{mStepA}>\mathrm{mPolyOrder})\{$

$\mathrm{mDataOk}=$ true;

mStepA $-=$ mPolyOrder;

mStepB $-=$ mPolyOrder;

\}

;

// Just do Calculations if we already got enough data points

if $(\mathrm{mDataOk}==$ true $)\{$

// Calculate the Polynomial Approximation to the fisrt estimator

//std::cout « "Step $=\ll<\mathrm{mStep}[0]$ « std::endl;

mpPolyGetsIt $=$ mpPolyGets $+($ mPolyOrder +1$) *($ mPolyOrder +1$) * m S t e p A ;$

mpPolyCoefsIt $=$ mpPolyCoefs;

for (it_i $=0$; it_i<=mPolyOrder; it_i++) \{

$*$ mpPolyCoefsit $=0$;

mpInputBufferIt $=$ mpInputBuffer;

for (it $\_\mathrm{j}=0 ;$ it $\_\mathrm{j}<=$ mPolyOrder; it $\left.\mathrm{j}++\right)\{$

//printf("PolyGets[\%d, \%d] = \%10.10Lff\{\}n",it_i, it_j, *mpPolyGetsIt);

//std::cout « "InputBuffer[i, j] = «<*mpInputBufferIt « std::endl; 


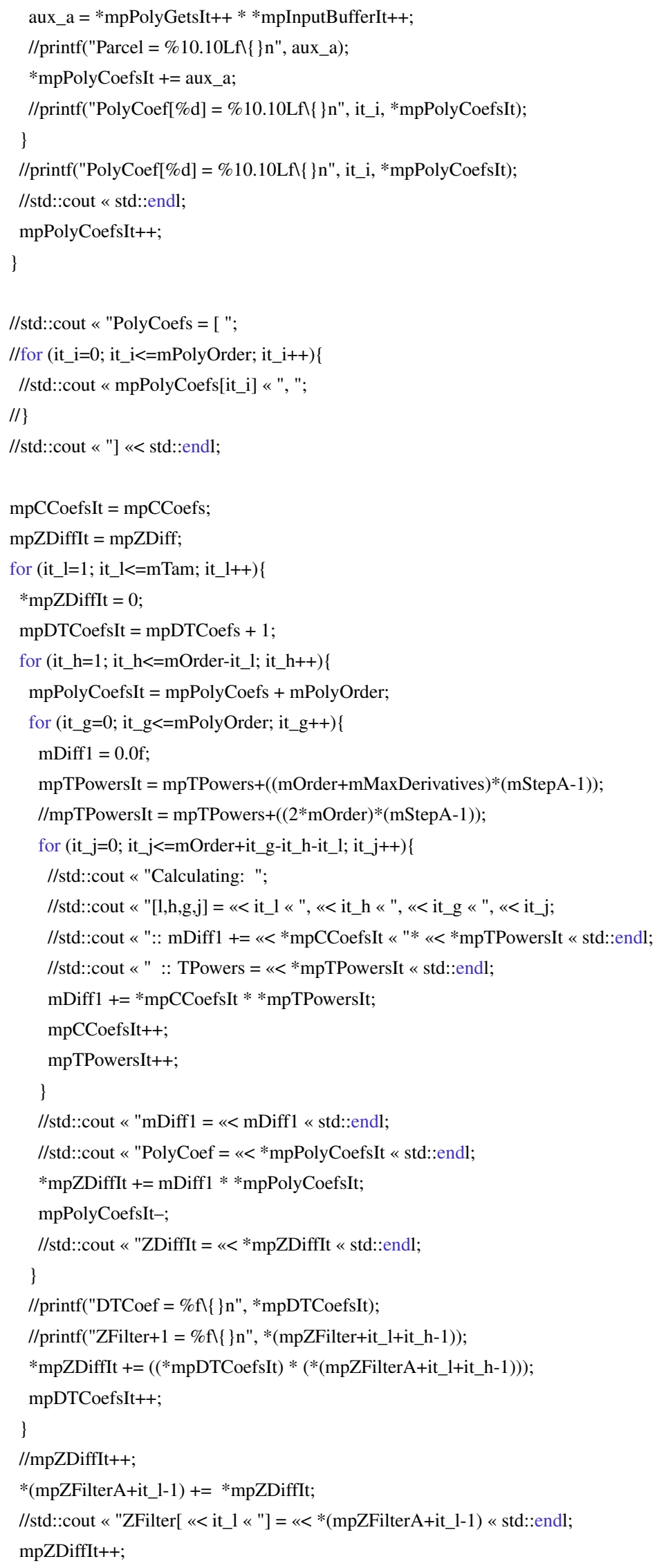

// Same for estimator B 


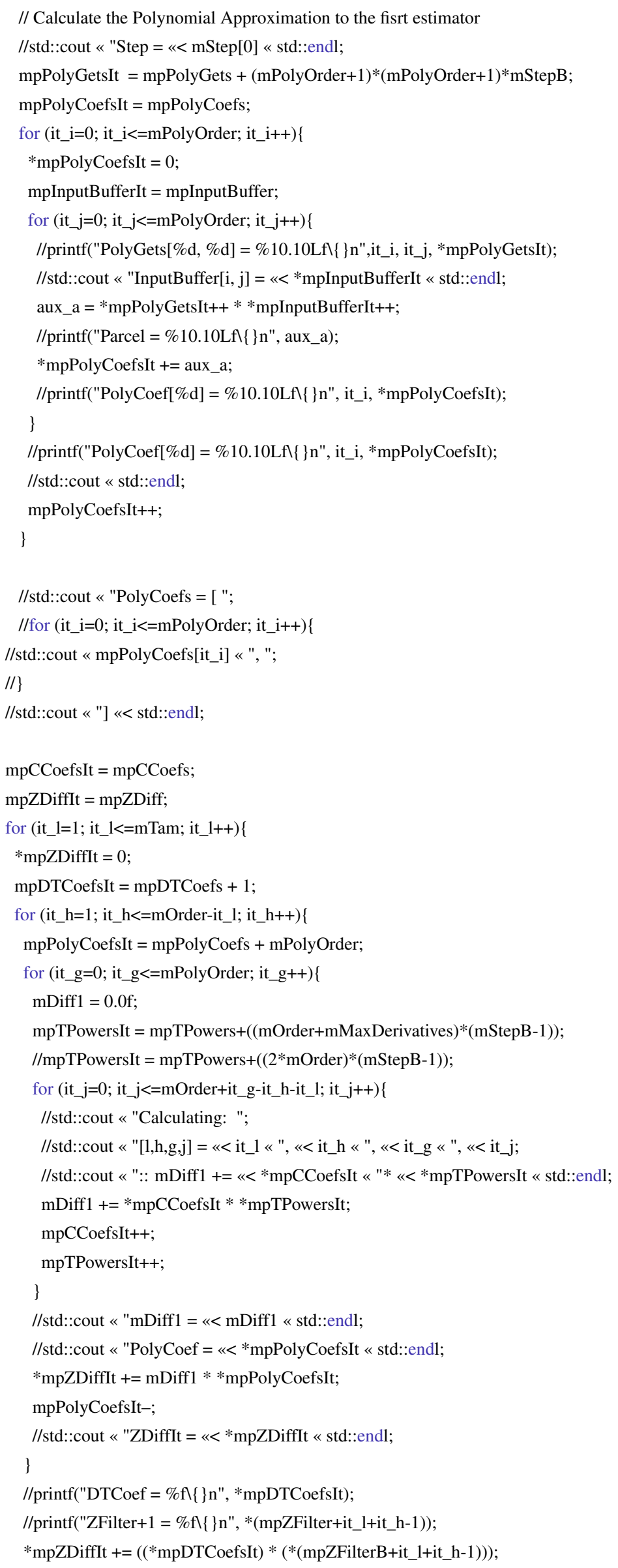




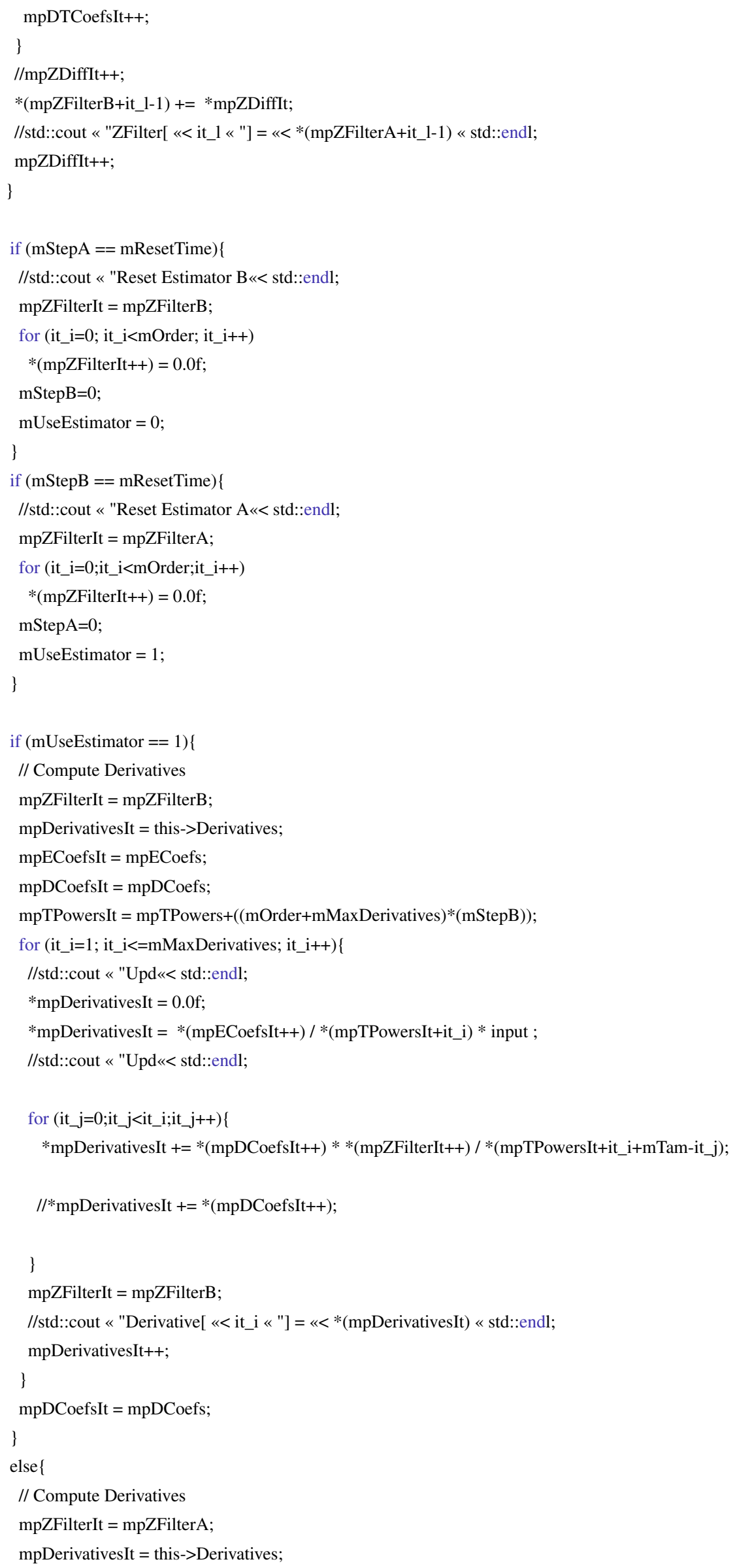




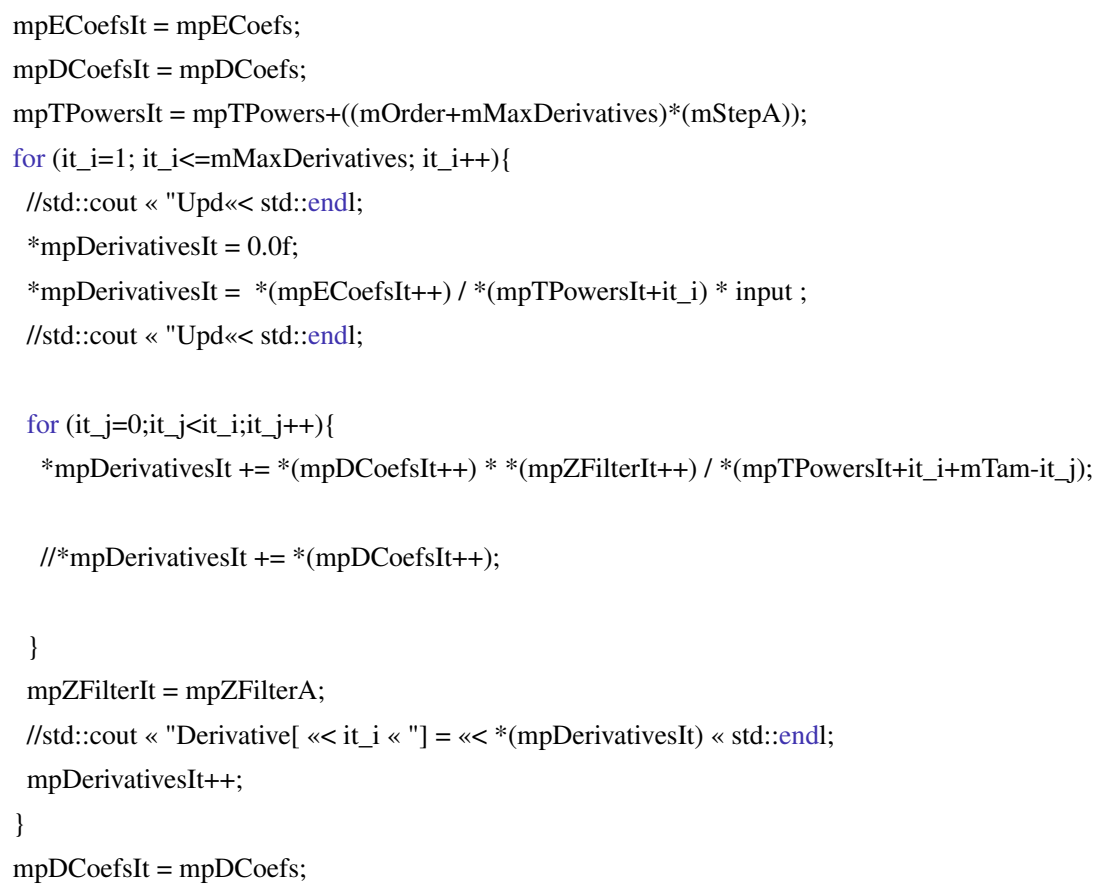




\section{Arquivo matrix.h}

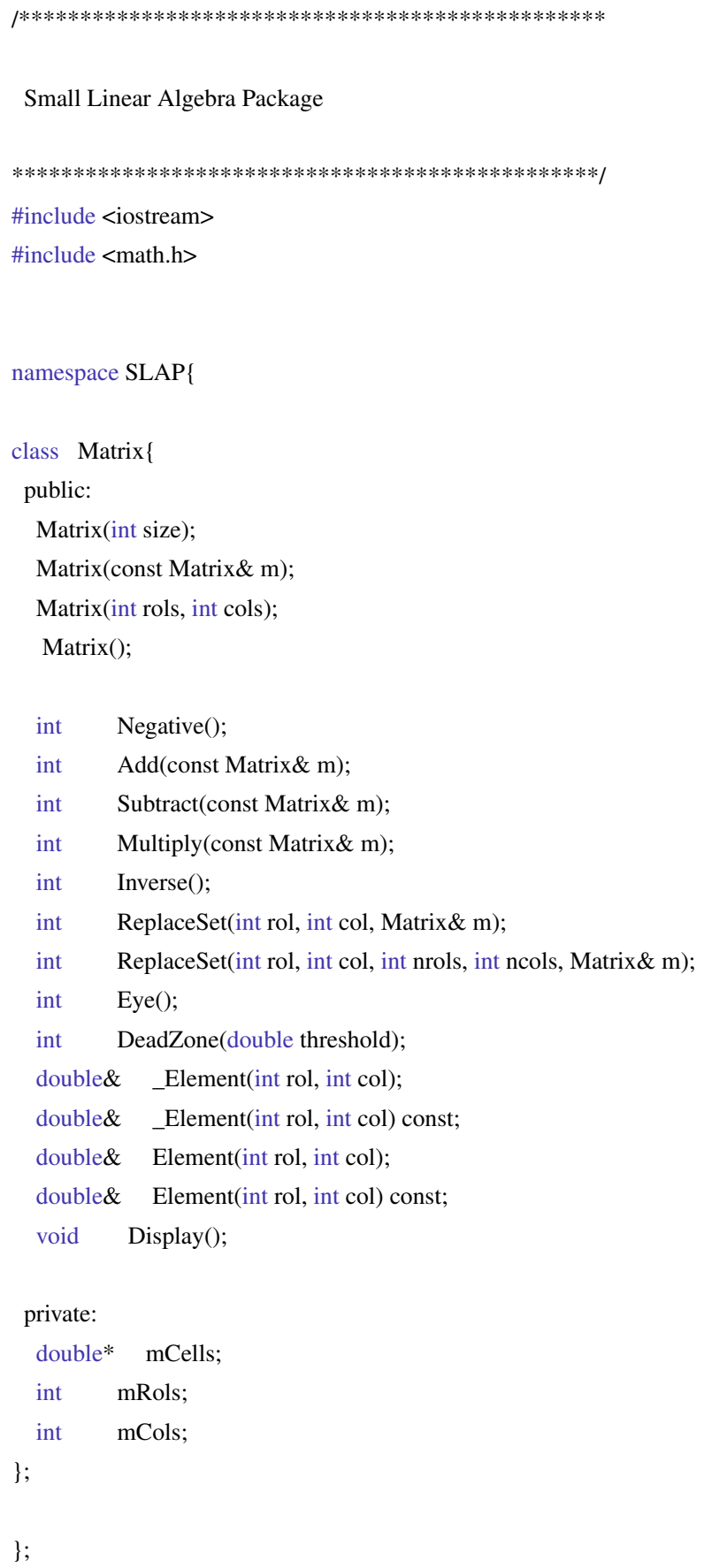




\section{Arquivo matrix.cpp}

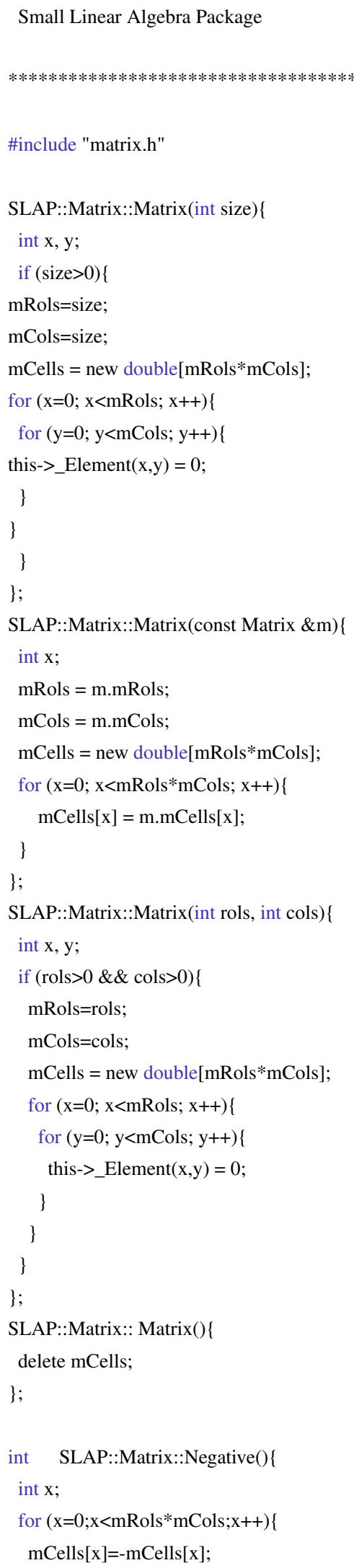




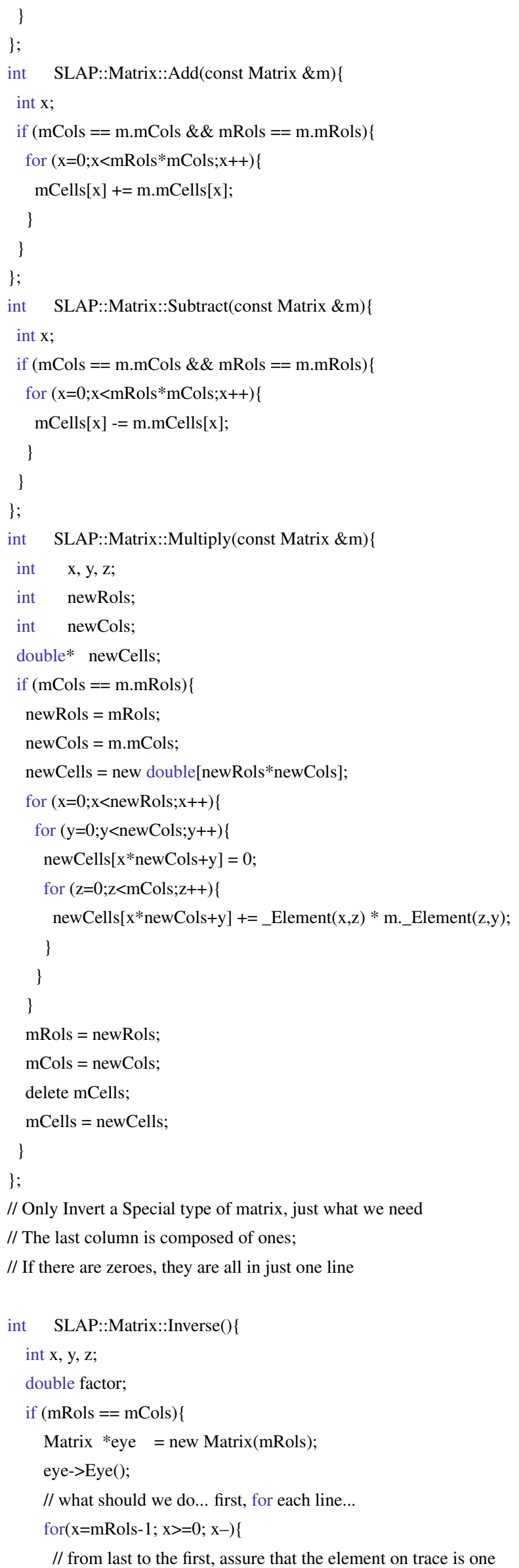




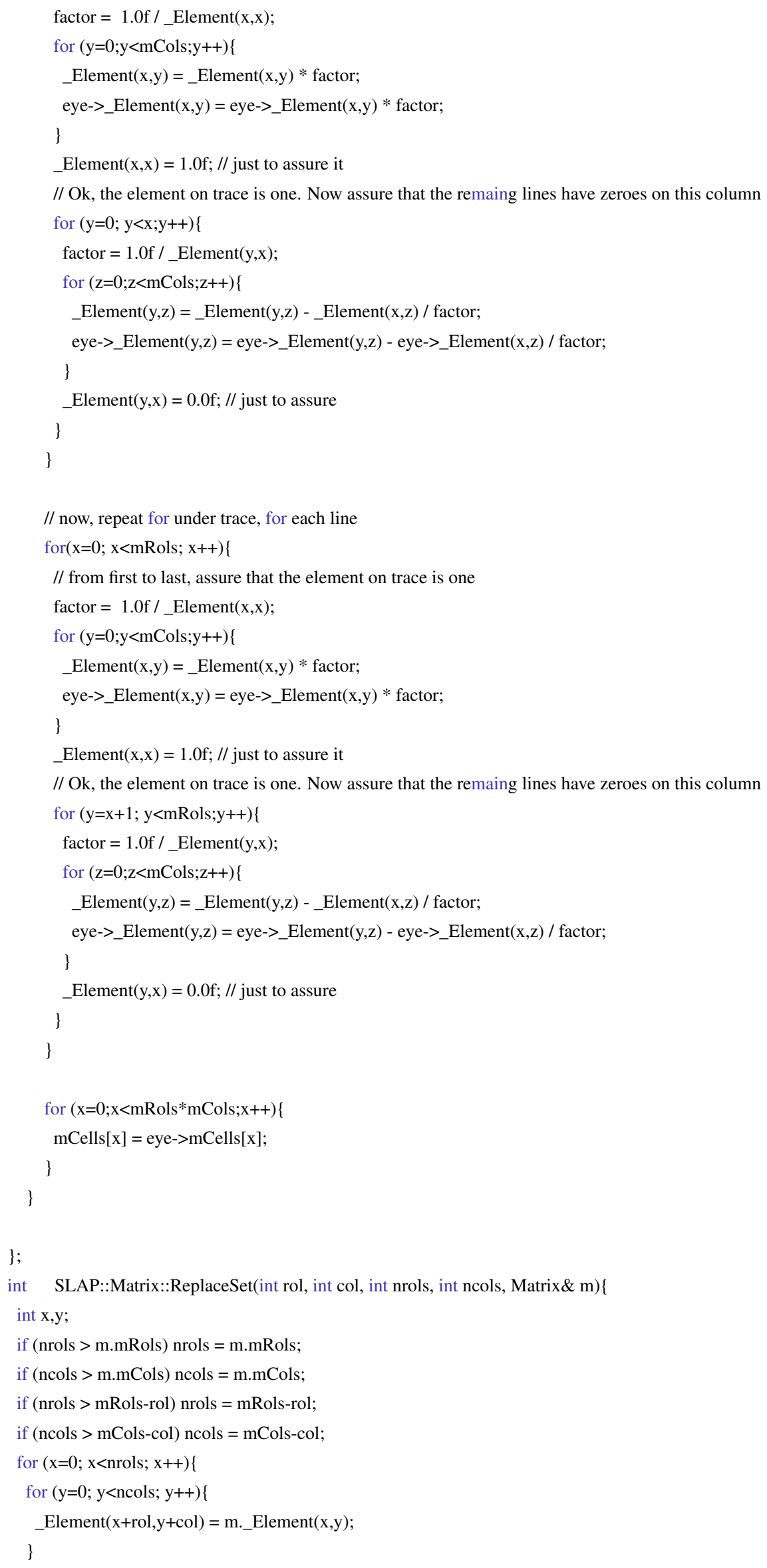




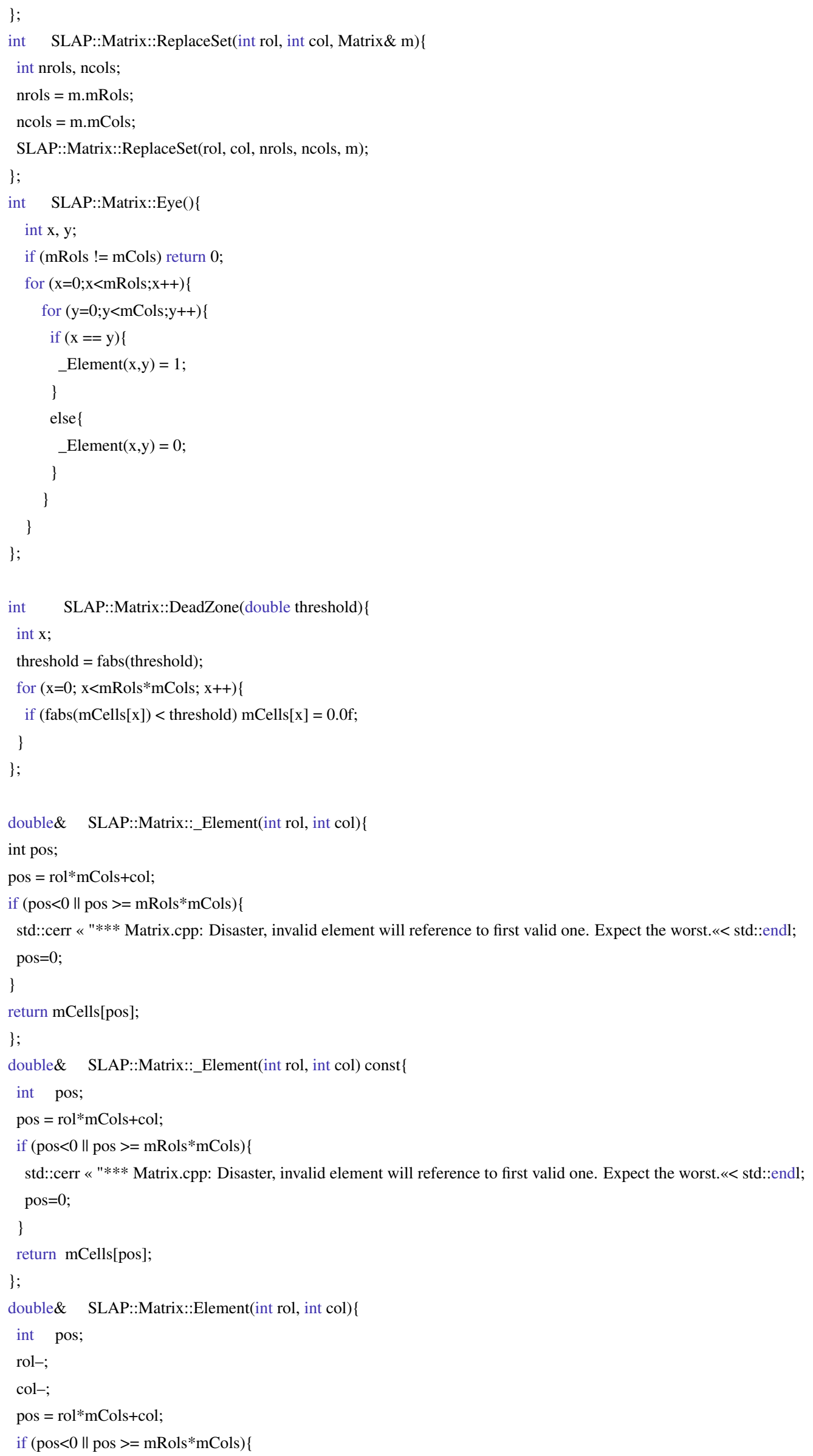




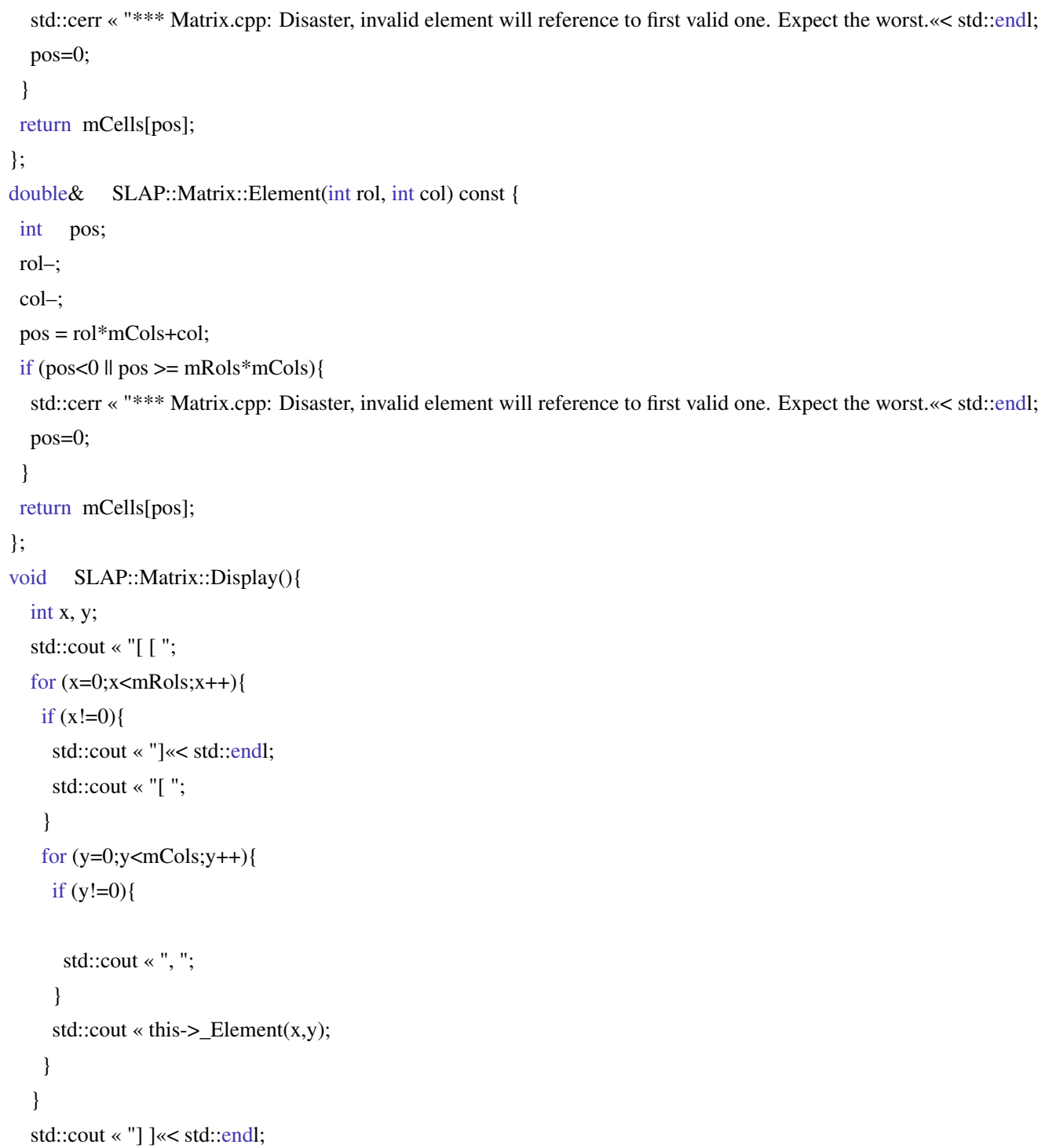




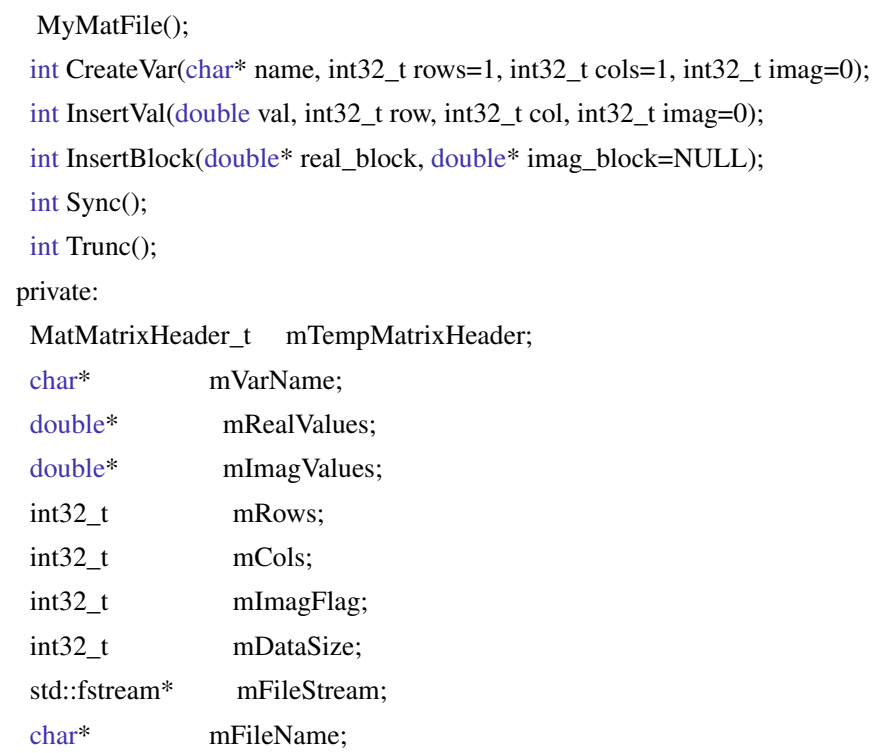




\section{Arquivo mymat.cpp}

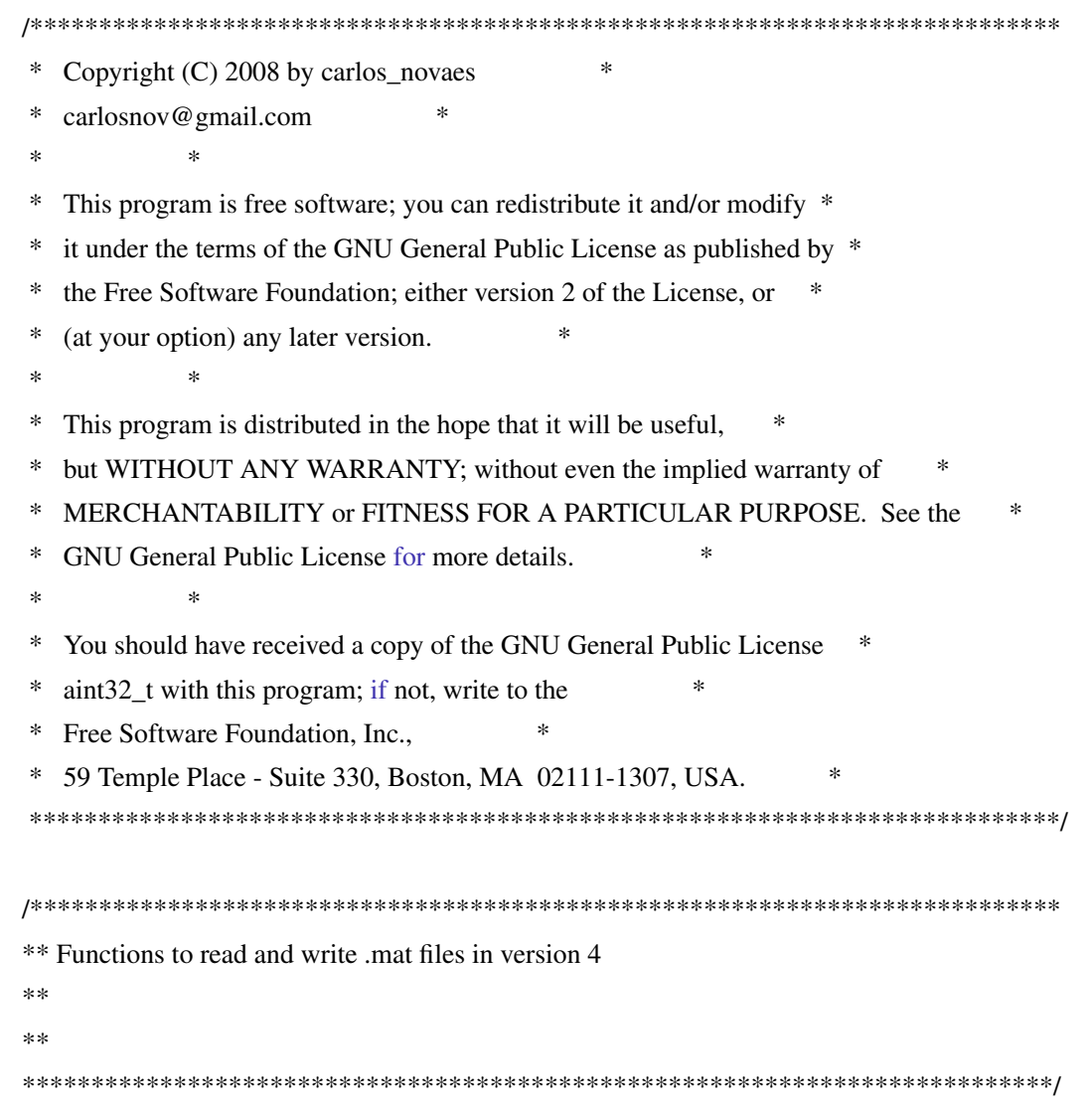

\#include "mymat.h"

\#include <iostream>

\#include $<$ cstring $>$

MyMatFile::MyMatFile(char* fname)\{

mFileName $=$ new char $[\operatorname{strlen}($ fname $)+1]$;

strcpy(mFileName, fname);

mVarName $==$ NULL;

mRealValues $==$ NULL;

mImagValues $==$ NULL;

\} ;

MyMatFile:: MyMatFile()\{

\};

int MyMatFile::CreateVar(char* name, int32_t rows, int32_t cols, int32_t imag $)\{$

mDataSize $=$ rows $*$ cols;

mImagFlag = imag;

if $(\mathrm{mVarName}==\mathrm{NULL})\{$

delete mVarName;

mVarName $==$ NULL;

\}

if $(\mathrm{mRealValues}==\mathrm{NULL})\{$

delete mRealValues;

mRealValues $==$ NULL;

\} 


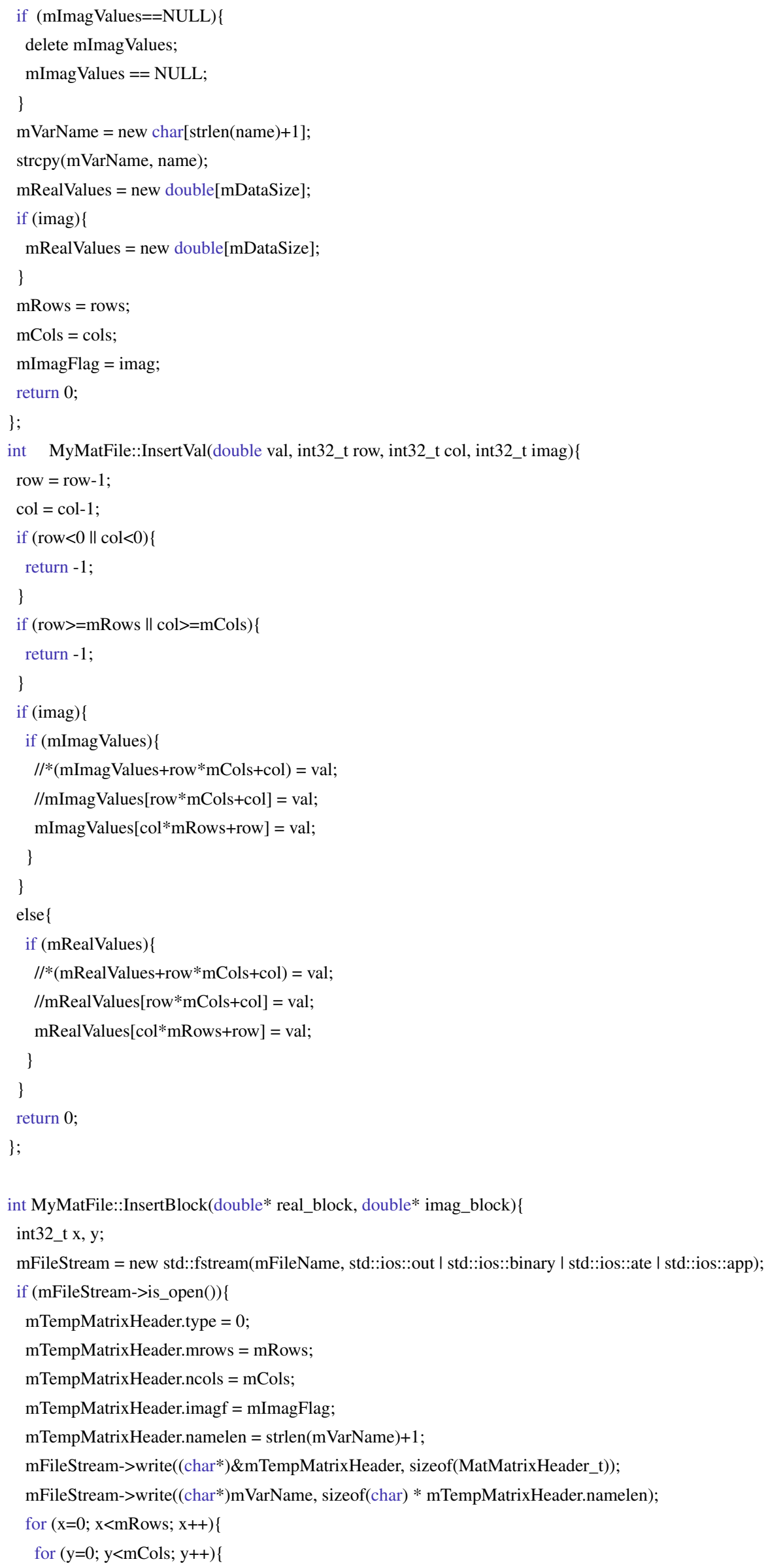




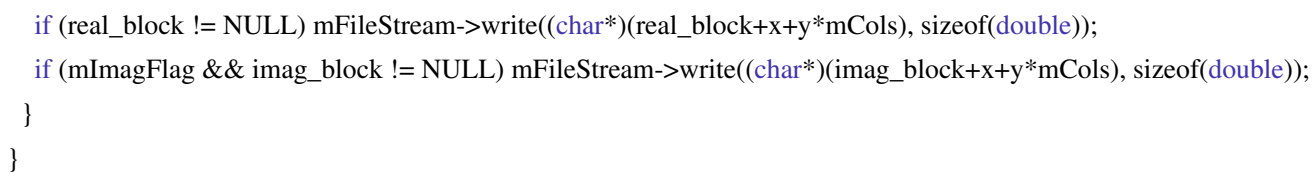

// End Of File 


\begin{tabular}{|c|c|c|}
\hline \#define & LYNX_EXPANSION_4 & LYNX_BASEADDR +12 \\
\hline \#define & LYNX_EXPANSION_5 & LYNX_BASEADDR + 13 \\
\hline \#define & LYNX_EXPANSION_6 & LYNX_BASEADDR + 14 \\
\hline \#define & LYNX_EXPANSION_7 & LYNX_BASEADDR + 15 \\
\hline \#define & LYNX_SECLIM & 0 \\
\hline \#define & LYNX_SECPOINTER & 1 \\
\hline \#define & LYNX_SECCOMAD & 2 \\
\hline \#define & LYNX_SECRM & 3 \\
\hline \#define & LYNX_SECMEM & 4 \\
\hline \#define & LYNX_SECACAL & 6 \\
\hline \#define & LYNX_BIPOLAR & $0 \times 80$ \\
\hline \#define & LYNX_UNIPOLAR & $0 \times 00$ \\
\hline \#define & LYNX_G1 & \\
\hline \#define & LYNX_G2 & \\
\hline \#define & LYNX_G3 & \\
\hline \#define & LYNX_G100 & \\
\hline
\end{tabular}

//\#include <stdint.h>

// End Of Card Specific Data

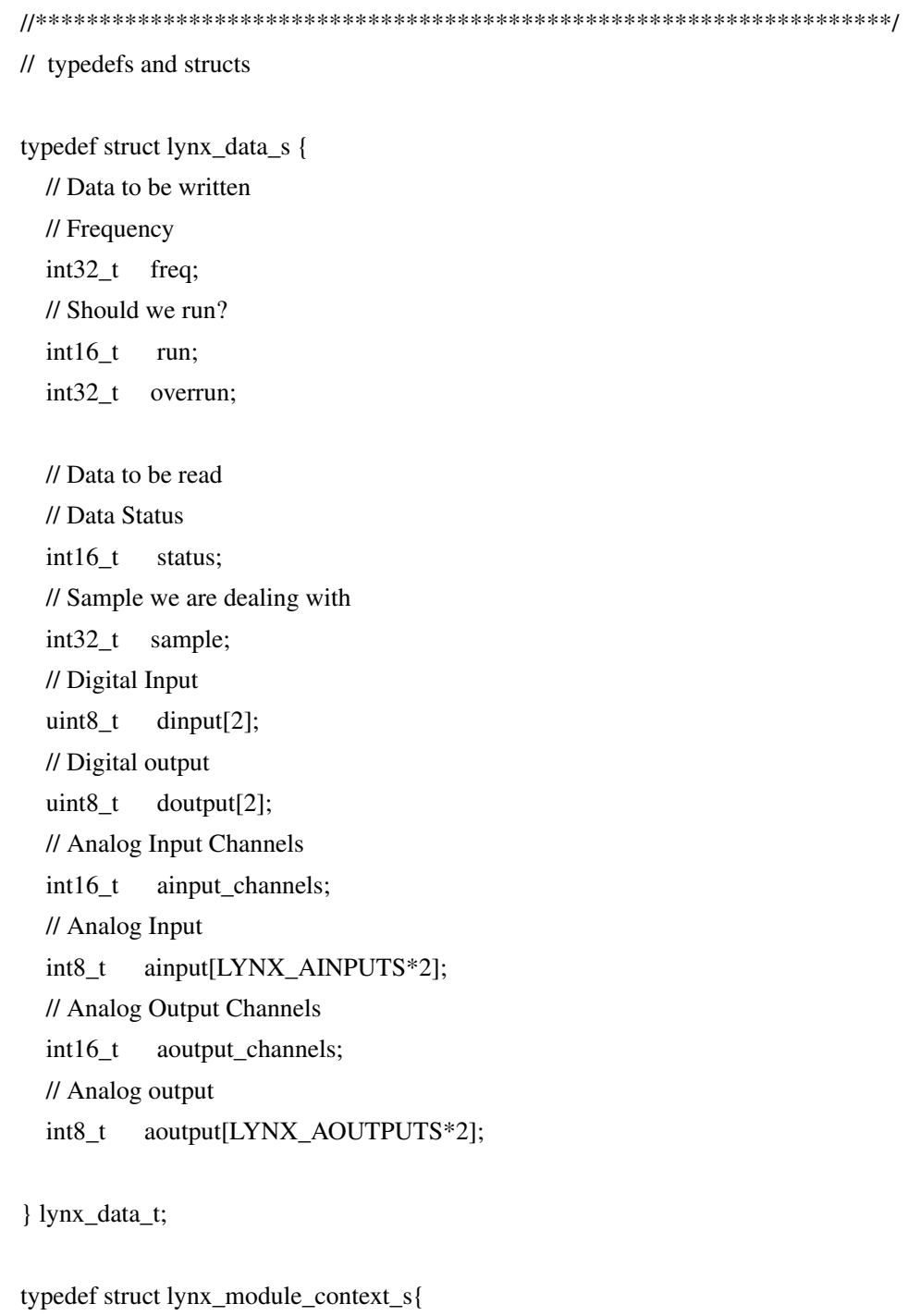




$\begin{array}{ll}\text { RT_HEAP } & \text { heap_desc; } \\ \text { RT_EVENT } & \text { event_desc; } \\ \text { RT_TASK } & \text { task_desc; } \\ \text { RT_INTR } & \text { intr_desc; } \\ \text { lynx_data_t } & \text { *data; }\end{array}$

\} lynx_module_context_t;

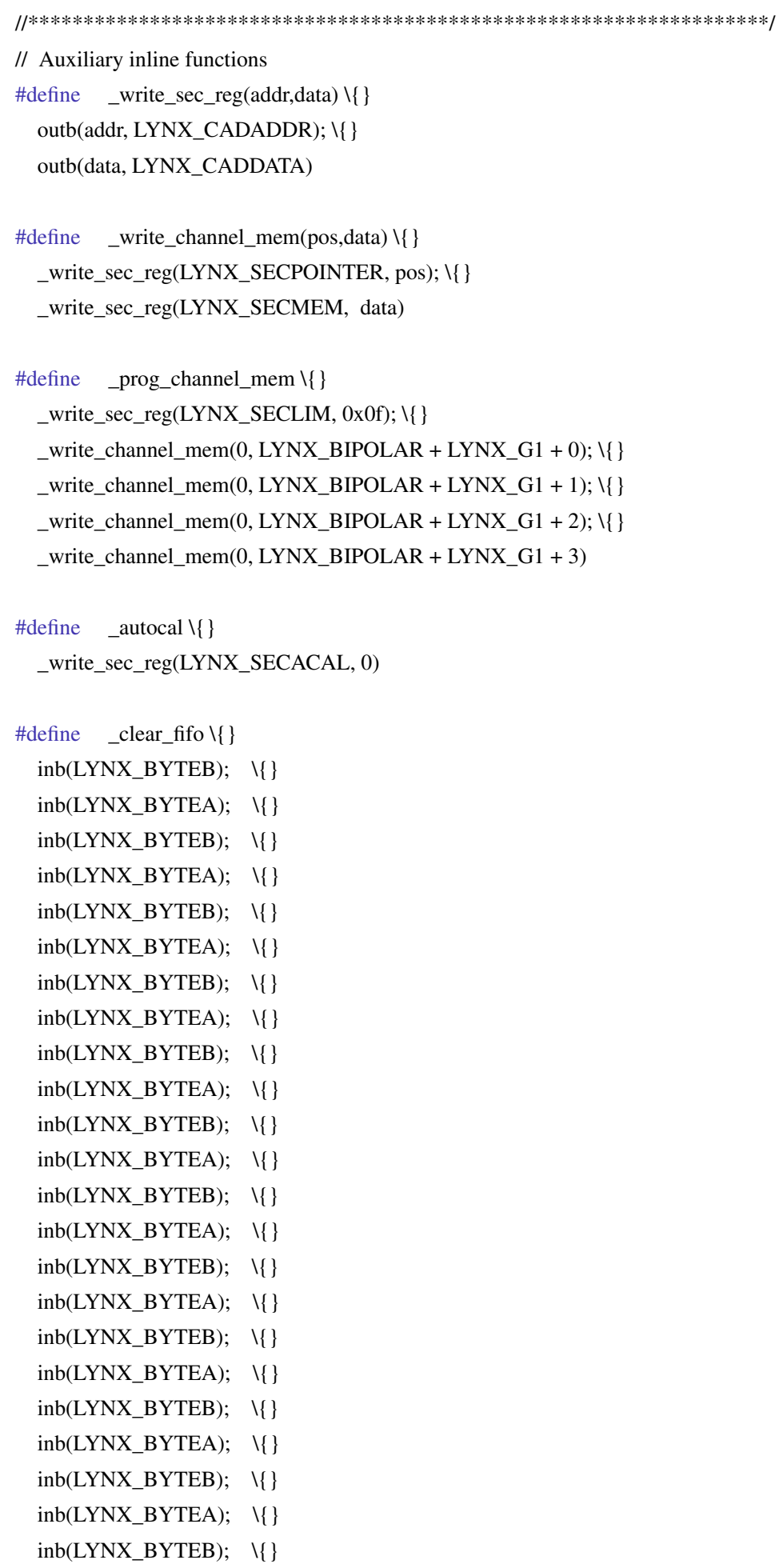


$\begin{array}{lc}\text { inb(LYNX_BYTEA); } & \backslash\{\} \\ \text { inb(LYNX_BYTEB); } & \backslash\{\} \\ \text { inb(LYNX_BYTEA); } & \backslash\{\} \\ \text { inb(LYNX_BYTEB); } & \backslash\{\} \\ \text { inb(LYNX_BYTEA); } & \backslash\{\} \\ \text { inb(LYNX_BYTEB); } & \backslash\{\} \\ \text { inb(LYNX_BYTEA); } & \backslash\{\} \\ \text { inb(LYNX_BYTEB); } & \backslash\{\} \\ \text { inb(LYNX_BYTEA) } & \end{array}$

\#define _prog_timer(counter,mode,val) $\backslash\{\}$

outb(counter*64+48+mode*2, LYNX_CADMODE); $\backslash\{\}$

outb(val\%256, LYNX_BASEADDR+counter); $\backslash\{\}$

outb(val»8, LYNX_BASEADDR+counter)

\#define__disable_intgate $\backslash\{\}$

_write_sec_reg(LYNX_SECRM, 0x0c)

\#define_enable_intgate $\backslash\{\}$

_write_sec_reg(LYNX_SECRM, 0x4c) 


\section{Arquivo lynx.c:}

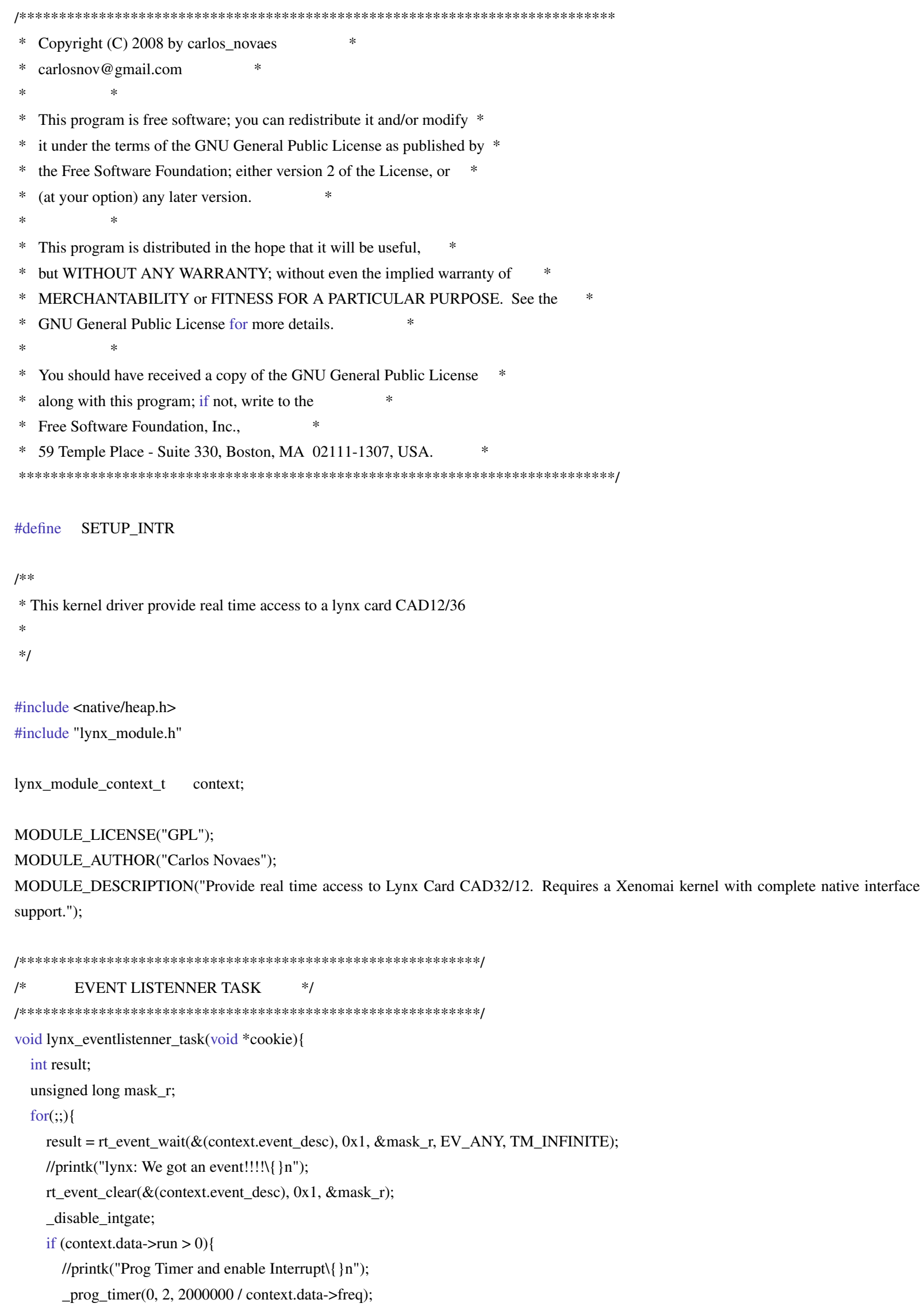




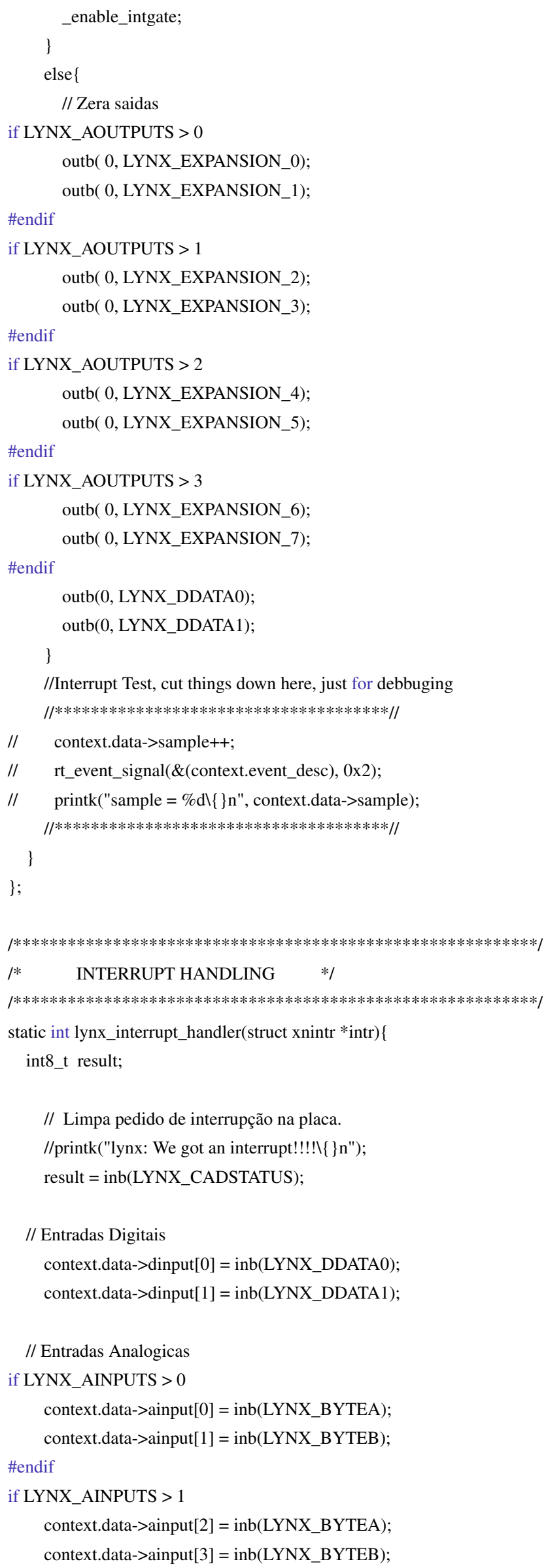


\#endif

if LYNX_AINPUTS > 2

context.data->ainput[4] = inb(LYNX_BYTEA);

context.data->ainput[5] = inb(LYNX_BYTEB);

\#endif

if LYNX_AINPUTS > 3

context.data->ainput[6] = inb(LYNX_BYTEA);

context.data->ainput[7] = inb(LYNX_BYTEB);

\#endif

if LYNX_AINPUTS > 4

context.data->ainput[8] = inb(LYNX_BYTEA);

context.data->ainput[9] = inb(LYNX_BYTEB);

\#endif

if LYNX_AINPUTS > 5

context.data->ainput[10] = inb(LYNX_BYTEA);

context.data->ainput[11] = inb(LYNX_BYTEB);

\#endif

if LYNX_AINPUTS > 6

context.data->ainput[12] = inb(LYNX_BYTEA);

context.data->ainput[13] = inb(LYNX_BYTEB);

\#endif

if LYNX_AINPUTS > 7

context.data->ainput[14] = inb(LYNX_BYTEA);

context.data->ainput[15] = inb(LYNX_BYTEB);

\#endif

if LYNX_AINPUTS > 8

context.data->ainput[16] = inb(LYNX_BYTEA);

context.data->ainput[17] = inb(LYNX_BYTEB $)$;

\#endif

if LYNX_AINPUTS > 9

context.data->ainput[18] = inb(LYNX_BYTEA);

context.data->ainput[19] = inb(LYNX_BYTEB);

\#endif

if LYNX_AINPUTS > 10

context.data->ainput[21] = inb(LYNX_BYTEA);

context.data->ainput[22] = inb(LYNX_BYTEB $)$;

\#endif

if LYNX_AINPUTS > 11

context.data->ainput[23] = inb(LYNX_BYTEA);

context.data->ainput[24] = inb(LYNX_BYTEB);

\#endif

if LYNX_AINPUTS > 12

context.data->ainput[25] = inb(LYNX_BYTEA);

context.data->ainput[26] = inb(LYNX_BYTEB);

\#endif

if LYNX_AINPUTS > 13

context.data->ainput[27] = inb(LYNX_BYTEA);

context.data->ainput[28] = inb(LYNX_BYTEB);

\#endif

if LYNX_AINPUTS > 14

context.data->ainput[29] = inb(LYNX_BYTEA);

context.data->ainput[30] = inb(LYNX_BYTEB);

\#endif

if LYNX_AINPUTS > 15 


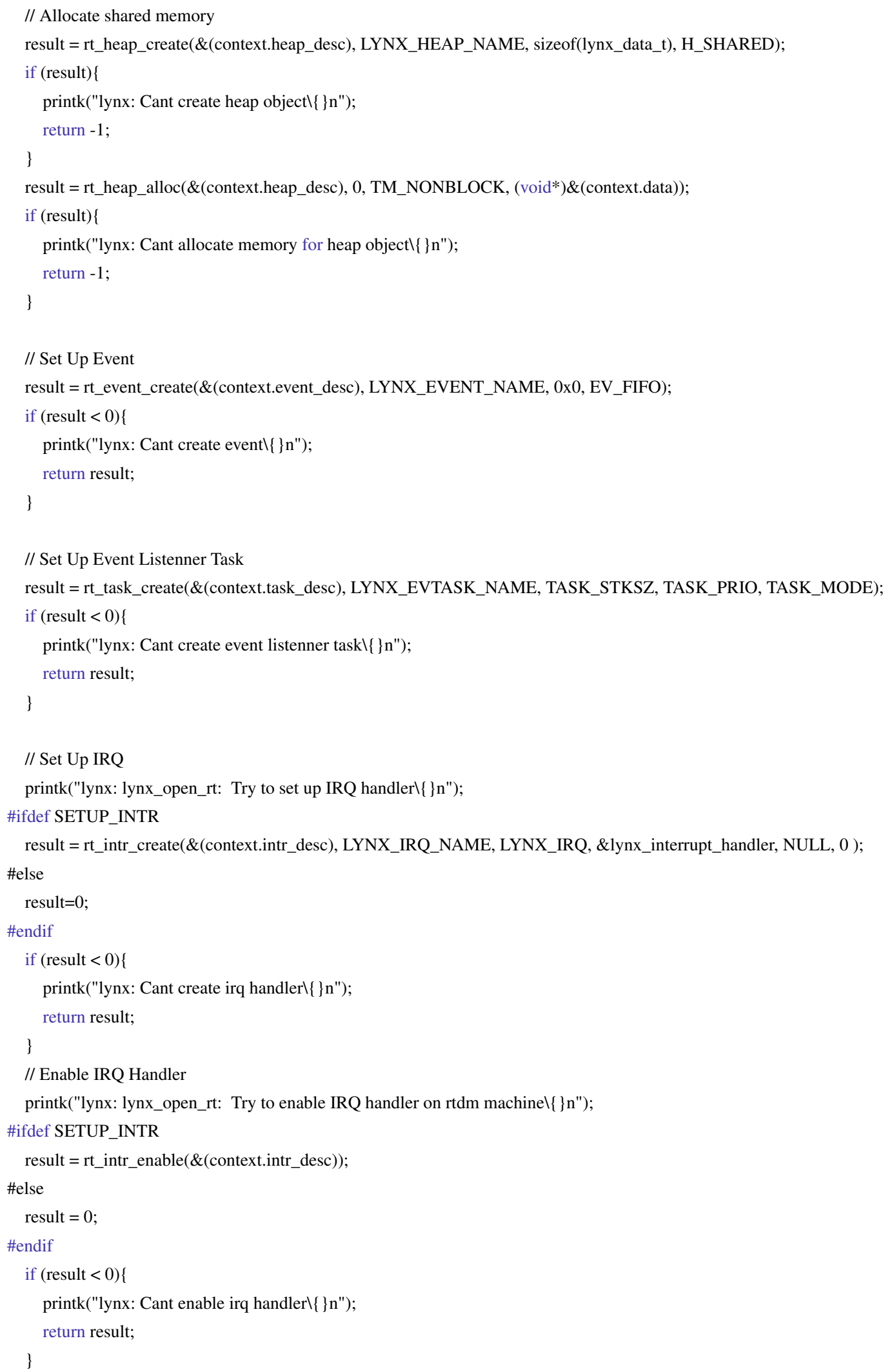


context.data- $>$ sample $=0$;

context.data->ainput_channels $=$ LYNX_AINPUTS;

context.data- $>$ aoutput_channels $=$ LYNX_AOUTPUTS;

// Set Up Card

printk("lynx: 1ynx_open_rt: Try to set up card \{\}$n$ ");

//EscreveRegSecundario (SecRM, 0).

_write_sec_reg(LYNX_SECRM, 0);

// Set Up sampling frequency

result $=2000000 /$ context .data- $>$ freq;

_prog_timer(0, 2, result);

// Autocal

_autocal;

//ProgramaMemoriaCanais;

_prog_channel_mem;

//EscreveRegSecundario (SecLimite, LYNX_AINPUTS-1);

_write_sec_reg(LYNX_SECLIM, LYNX_AINPUTS-1);

//EscreveRegSecundario (SecPonteiro, 0);

_write_sec_reg(LYNX_SECPOINTER, 0);

//LimpaFIFO;

_clear_fifo;

inb(LYNX_CADSTATUS);

// Start Event Listenner Task

result $=$ rt_task_start $\left(\&(\right.$ context.task_desc $), \& l y n x \_$eventlistenner_task, NULL $)$;

printk("lynx: Module Inserted \{\} n");

// ************

return 0 ;

\}

/************************************************************/

/* CLEANUP DRIVER */

/***************************************************************/

void cleanup_module(void)

\{

printk("lynx: Removing Modulel\{\}n");

// Free Task

rt_task_delete(\&(context.task_desc));

// Free Event

rt_event_delete(\&(context.event_desc)); 
// remove irq handler; \#ifdef SETUP_INTR

rt_intr_disable(\&(context.intr_desc));

rt_intr_delete(\&(context.intr_desc));

\#endif

// Free Heap

rt_heap_free(\&(context.heap_desc), \&(context.data));

rt_heap_delete(\&(context.heap_desc)); 


\title{
Arquivo lynx.h:
}

\author{
\#include <native/heap.h> \\ \#include <native/event.h> \\ \#include $<$ native/intr.h> \\ \#include $<$ native/task.h $>$ \\ \#include <stdint.h>
}

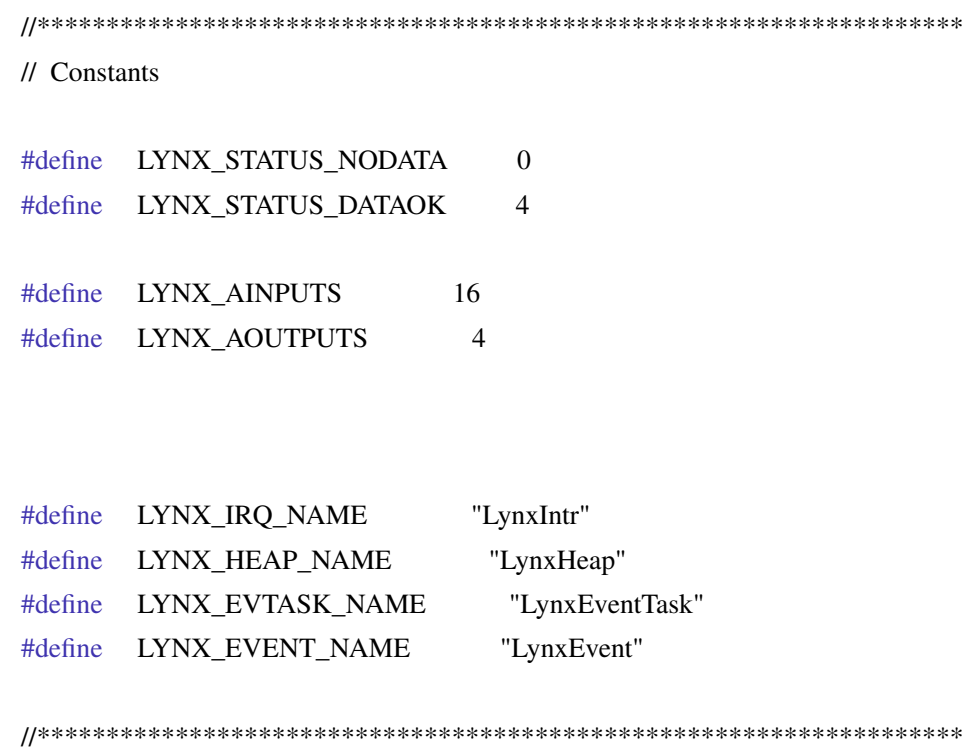

typedef struct lynx_data_s \{

// Data to be written

// Frequency

int32_t freq;

// Should we run?

int16_t run;

int32_t overrun;

// Data to be read

// Data Status

int16_t status;

// Sample we are dealing with

int32_t sample;

// Digital Input

//uint8_t dinput[2];

int16_t dinput;

// Digital output

//uint8_t doutput[2];

uint16_t doutput;

// Analog Input Channels

int16_t ainput_channels;

// Analog Input

//int8_t ainput[LYNX_AINPUTS][2];

int16_t ainput[LYNX_AINPUTS];

// Analog Output Channels

int16_t aoutput_channels;

// Analog output 
//int8_t aoutput[LYNX_AOUTPUTS][2];

int16_t aoutput[LYNX_AOUTPUTS];

\} lynx_data_t; 